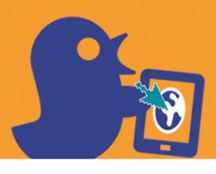

\title{
COME IL
}

MONDO HA

\section{CAMBIATO I}
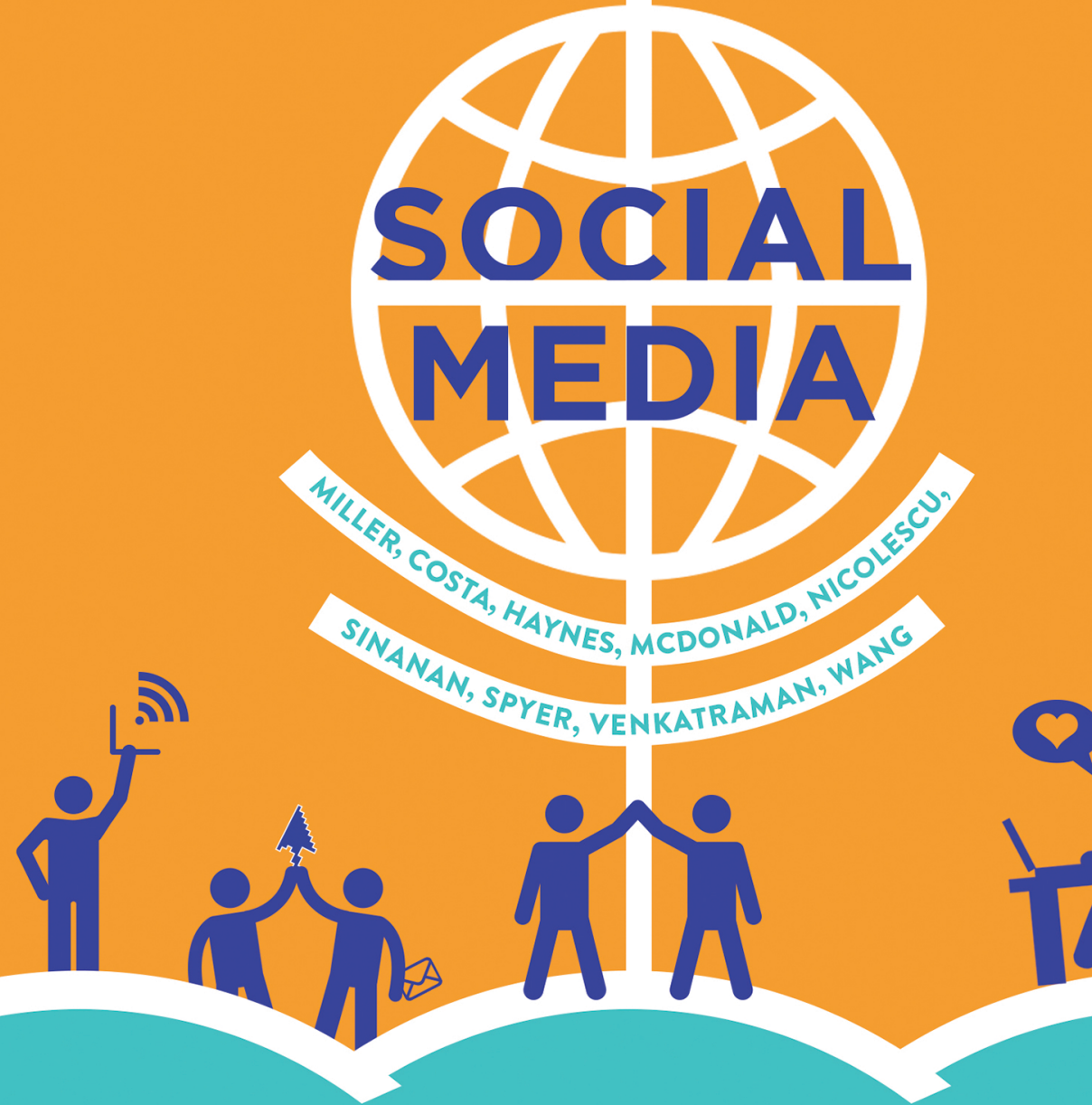

^UCLPRESS 


Come il mondo ha cambiato i social media 

Daniel Miller, Elisabetta Costa, Nell Haynes, Tom McDonald, Razvan Nicolescu, Jolynna Sinanan, Juliano Spyer, Shrimam Venkatraman, Xinyuan Wang

\title{
Come il mondo ha cambiato i social media
}

\author{
Edizione italiana a cura di \\ Gabriella D'Agostino e Vincenzo Matera
}


Questa edizione open access viene pubblicata nel 2019 da UCL Press

University College London

Gower Street

London WC1E 6BT

Disponibile per il download gratuito: www.ucl.ac.uk/ucl-press

Pubblicato in Italia da Ledizioni LediPublishing:

ISBN: 978-88-6705-783-2 (print)

ISBN: 978-88-6705-823-5 (ebook)

Testo (C) Daniel Miller, Elisabetta Costa, Nell Haynes, Tom McDonald, Razvan Nicolescu, Jolynna Sinanan, Juliano Spyer and Shriram Venkatraman 2016 Images (C) Daniel Miller, Elisabetta Costa, Nell Haynes, Tom McDonald, Razvan Nicolescu, Jolynna Sinanan, Juliano Spyer and Shriram Venkatraman 2016

Una registrazione CIP per questa libro è disponibile presso la British Library.

Questo libro è pubblicato con una licenza Creative Commons Attribution Non-commercial Non-derivative 4.0 International license (CC BY-NC-ND 4.0). Questa licenza ti permette di riprodurre, distribuire, comunicare al pubblico, esporre in pubblico, rappresentare, eseguire e recitare questo materiale con qualsiasi mezzo e format, a patto di riconoscere una menzione di paternità adeguata, di non utilizzare il materiale per scopi commerciali e di non distribuire il materiale modificato. Maggiori dettagli sulle licenze CC BY sono disponibili qui: http://creativecommons.org/licenses/by/4.0

ISBN: 978-1-78735-557-6 (PDF)

DOI: https://doi.org/10.14324/111.9781787355576 


\section{Indice}

Introduzione all'edizione italiana

di Gabriella D'Agostino e Vincenzo Matera

Come il mondo ha cambiato i social media Introduzione alla serie Why we post

Capitoli introduttivi

1. Che cosa sono i social media

2. Lo studio accademico dei social media

3. Metodo e approccio

4. I risultati della nostra indagine

I dieci temi chiave

5. Educazione e giovani

6. Lavoro e commercio

7. Relazioni online e offline

8. Genere

9. Disuguaglianza

10. Politica

11. Immagini visive

12. Individualismo 
13. I social media rendono la gente più felice?

14. Il futuro

Note

Bibliografia

Sitografia

Sintesi dei contenuti

Elenco delle immagini

Elenco delle tabelle

Elenco dei collaboratori

Indice degli argomenti 


\section{Introduzione all'edizione italiana*}

Come il mondo ha cambiato i social media è il volume complessivo di comparazione dei risultati di un'ampia indagine etnografica, coordinata da Daniel Miller, dall'eloquente titolo Why we Post. Nove ricercatori, incluso Miller, hanno trascorso 15 mesi sul campo, in diversi paesi del mondo (Italia del sud, Turchia sudorientale, due siti in Cina, area rurale e area industriale, Trinidad, Inghilterra, India del sud, Cile settentrionale e Brasile) a osservare e studiare, con un approccio etnografico, i modi in cui le persone usano i social media. È un fatto indiscutibile che i social sono entrati nella nostra vita con prepotenza, in modo capillare, per certi aspetti invasivo. Con un linguaggio fluido, talvolta anche colloquiale, il lettore è condotto all'interno di un ambito che gli sembra di conoscere, se non altro perché ne siamo tutti, più o meno, utenti, scoprendo però quanto di valori, di comportamenti culturalmente codificati, di 'polizia morale' ci sia dentro i social media. L'approccio qui presentato parte infatti da un'idea un po' diversa rispetto a quelle più diffuse, e avvalorata nel corso della ricerca: se è indubbio che i social media hanno cambiato il mondo, la questione più interessante riguarda però il modo in cui il mondo li ha cambiati.

Grazie alla prospettiva antropologica che sostiene la ricerca e al metodo etnografico, i ricercatori hanno riconosciuto una costante in tutti i siti indagati: le strategie e le modalità di adattamento che ciascun contesto ha rivelato rispetto all'uso dei social media, il fatto che in questo spazio online si possono cogliere dinamiche culturali, proprie di gruppi di popolazione e di sottogruppi interni, fondate e articolate su prospettive e valori condivisi. La postura antropologica corroborata dall'approccio etnografico smonta dunque tanti luoghi comuni sulle conseguenze della tecnologia, in particolare di quella digitale: il timore diffuso, per esempio, che il tempo trascorso a digitare con uno smartphone sia tempo sottratto alla socialità e incrementi una solitudine alienante; l'idea che il dilagare di social, con chat, messaggistica, profili, immagini post, video, ecc. ci renda meno pronti cognitivamente, più passivi esecutori di azioni già predisposte; l'idea, ancora, che l'uso dei social media incrementi le tendenze già forti nelle società contemporanee a un individualismo esasperato. Discorsi e considerazioni ricorrenti, luoghi comuni impermeabili al principio, in fondo ovvio, che i social media, per 
definizione, si muovono in ambiente sociale, da esso prendono forma, a esso si alimentano. Tenendo conto delle condizioni storiche e culturali in cui il fenomeno emerge, secondo il procedimento 'decostruttivo' classico della riflessione antropologica ampio spazio della riflessione riguarda la relazione tra vita offline e online. Senza negare il potere straordinario dei social media di annullare, o ridurre, dimensioni spaziali e temporali prima difficilmente colmabili, modalità di comportamento online e offline sono molto più sovrapponibili di quanto a prima vista non si sia portati a pensare. I social e tutte le interazioni che realizziamo per loro tramite sono parte della nostra vita complessiva, proprio come le conversazioni telefoniche, che nessuno si sognerebbe di considerare ormai come un ambito a parte e separato rispetto alle conversazioni faccia a faccia. Questa è la linea corretta, secondo gli autori, da cui guardare al fenomeno dei social, al loro significato culturale, e alle conseguenze sociali della loro diffusione.

Il libro, articolato in quattro capitoli di carattere generale, propone una serie di intrecci della questione principale (l'uso dei social media) declinandola in dieci tematiche, centrali delle scienze sociali e dell'antropologia, e che segnano problemi a volte drammatici del mondo attuale. Politica, Genere, Individualismo, Disuguaglianza sono alcuni di questi temi e mostrano come in modi estremamente variabili i social, per esempio, intervengano nella rappresentazione dell'uguaglianza, anche se questa può riguardare solo la dimensione online. Qui i profili possono essere equivalenti, si possono usare anche profili falsi, si può moltiplicare la propria identità. Non è difficile immaginare gli effetti di questa possibilità nelle relazioni di genere, e come possa risultarne favorito lo spazio di azione delle donne nell'interazione con persone estranee, laddove ciò è severamente vietato. Questo influisce anche sui più generali modelli di interazione sociale e sulla stessa possibilità di adesione multipla, e modulabile, a seconda delle circostanze e delle dinamiche che si intende perseguire. Resta difficile tuttavia individuare e immaginare rapporti diretti fra uso dei social in un certo contesto e processi di trasformazione sociale e culturale. Il caso delle recenti rivolte in alcuni paesi del Nord Africa ne è un chiaro esempio: i social hanno contribuito moltissimo a far circolare le notizie, a organizzare le proteste, a generare una sorta di riappropriazione della capacità di comunicazione fra le persone e questo è sicuramente una prova a favore dell'integrazione delle tecnologie digitali nella vita quotidiana. Quanto poi di fatto le strutture sociali gerarchiche di quelle società siano effettivamente cambiate o potranno cambiare è ancora 
presto per dirlo, e bisognerebbe darsi questo tema di ricerca nel prossimo futuro. Resta il fatto certo della capacità delle tecnologie digitali di esercitare un'enorme influenza sull'immaginazione delle persone, agevolando una risoluzione anche simbolica dei disagi e delle sofferenze. Se tuttavia vita online e vita offline non sono ambiti separati, la possibilità di agire concretamente sulle strutture di potere deve passare per l'una e per l'altra. Proprio per questo il libro che qui presentiamo in edizione italiana è importante e rappresenta un contributo alla ricerca e alla riflessione sui social media e sulla comunicazione digitale da punti di visti sinora inediti, pur nei limiti di un'indagine articolata su una scala ampia e comparativa, che compensa, nelle singole monografie, quanto qui non ha trovato spazio per specifici e puntuali approfondimenti di contesti. Una lettura che offre moltissimi spunti utili per chiunque volesse saperne di più su un fenomeno così pervasivo e così attuale, secondo una prospettiva disciplinare di tipo qualitativo che dialoga, nel senso più pieno e articolato del termine, con le donne e gli uomini della nostra contemporaneità.

Gabriella D’Agostino e Vincenzo Matera

*Gabriella D’Agostino insegna Antropologia culturale nell’Università degli Studi di Palermo, Dipartimento Culture e Società.

*Vincenzo Matera insegna Antropologia nell'Università degli Studi di Bologna, Dipartimento di Beni Culturali. 



\title{
Come il mondo ha cambiato i social media
}

\author{
Introduzione alla serie Why we post
}

Questo libro fa parte di una serie composta da 11 titoli. Nove sono monografie dedicate a campi specifici in Brasile, Cile, Cina, Inghilterra, India, Italia, Trinidad e Turchia. Saranno tutte pubblicate tra il 2016 e il 2017. Fanno parte della serie anche questo libro, il nostro lavoro di comparazione su tutto ciò che abbiamo scoperto, e un ultimo libro basato sul contrasto fra le immagini che le persone postano su Facebook rilevate nel nostro campo di ricerca in Inghilterra e in quello a Trinidad.

Dire che abbiamo scritto 9 monografie sui social media in tutto il mondo, utilizzando sempre gli stessi titoli di capitoli (con eccezione del capitolo 5), suscita una perplessità per il carattere potenzialmente ripetitivo di questa operazione. Tuttavia, nel caso decidiate di leggere alcuni di questi libri (come noi caldamente ci auguriamo), capirete che l'accorgimento è stato utile a dimostrare esattamente l'opposto. Ogni libro presenta una tale peculiarità e distinzione da apparire quasi come se trattasse di un argomento completamente diverso da quello di tutti gli altri.

Forse è proprio questa la nostra più importante scoperta. La maggior parte degli studi su internet e sui social media si basa su metodi di ricerca che danno per scontata la possibilità di generalizzare fra gruppi differenti. Osserviamo i tweet in un certo luogo e scriviamo su "Twitter". Testiamo il rapporto fra i social media e l'amicizia in una popolazione, e poi scriviamo su questo argomento come se "amicizia" significasse la stessa cosa per tutti. Proprio grazie ai nove libri con gli stessi titoli di capitoli, invece, sarete in grado di giudicare voi stessi quali generalizzazioni sono possibili e quali no.

La nostra intenzione non è valutare in positivo o in negativo i social media. Al contrario, lo scopo è di carattere istruttivo: documentare dettagliatamente che cosa sono diventati e che cosa provocano i social media in ciascun luogo, tenendo conto anche delle valutazioni locali.

Ogni libro si basa su 15 mesi di ricerca durante i quali la maggior parte degli antropologi ha vissuto, lavorato e interagito con le persone nella lingua locale. Essi tuttavia si differenziano dalla tradizione di scrittura dominante nella letteratura delle scienze sociali. Prima di tutto non si confrontano con 
la letteratura accademica sui social media. Sarebbe stato, questo sì, estremamente ripetitivo avere la stessa discussione in tutti e nove i libri. I dibattiti propri di tale letteratura si trovano qui, in questo singolo volume a carattere complessivo e comparativo. In secondo luogo, le monografie non presentano comparazioni, il che è invece il primo obiettivo del presente libro. Terzo, dato l'enorme interesse per i social media da parte di un pubblico ampio, abbiamo cercato di scrivere in uno stile accessibile e chiaro. Ciò significa che per le monografie si è scelto uno stile più diffuso nella storiografia, consistente nel tenere nelle note finali tutte le citazioni e tutte le discussioni che investono questioni accademiche più ampie.

$\mathrm{Ci}$ auguriamo che gli esiti vi piacciano e che siate anche indotti a leggere alcune monografie - oltre a questo sommario e al volume a carattere comparativo.

\section{Ringraziamenti}

I singoli autori ringraziano tutti coloro che li hanno aiutati nella loro ricerca all'interno dei rispettivi libri. In questo libro, desideriamo ringraziare il nostro principale finanziatore che è l'European Research Council SOCNET ERC-2011-AdG-295486. La partecipazione di Nell Haynes è finanziata dall'Interdisciplinary Center for Intercultural and Indigenous Studies di Santiago, Cile - ICIIS, CONICYT - FONDAP15110006. La partecipazione di Xinyuan Wang è finanziata dalla Wenner Grant Foundation.

Per i commenti sui singoli capitoli di questo libro, e non soltanto per questo, desideriamo ringraziare Justin Bourke, Isabel Colucci, Elijah Edelman, Augusto Fagundes, Marina Franchi, Nick Gadsby, Rebecca Stone Gordon, Thomas Haynes, Laura Haapio-Kirk, Sonia Livingstone, Omar Melo, Carolina Miranda, Jonathan Corpus Ong, Joowon Park, John Postil, Pascale Seale, Emanuel Spyer and Matthew Thomann.

Il presente libro è frutto di un lavoro collettivo, ma Xinyuan Wang merita una menzione speciale per aver realizzato tutte le schede grafiche. 
Capitoli introduttivi 



\section{Capitolo 1 Che cosa sono i social media'}

Molti degli studi precedenti sui social media sono dedicati a piattaforme specifiche, inclusi libri e articoli su una sola particolare piattaforma, come Facebook o Twitter ${ }^{2}$. È ovviamente importante studiare per esempio Twitter, in quanto piattaforma: l'azienda proprietaria, il modo in cui funziona e la sua particolarità di social media basato su messaggi che devono restare sotto i 140 caratteri. Da una prospettiva antropologica, tuttavia, se ci chiediamo che cosa concretamente sia Twitter ha più senso concentrarsi sui milioni di tweet, sui generi predominanti, sulle differenze regionali e sulle conseguenze emotive e sociali che ha per gli utenti. È il contenuto, e non la piattaforma, che assume maggiore significato quando si arriva a chiedersi perché i social media hanno importanza.

Come risulterà nelle nostre singole etnografie dei social media in tutto il mondo, tipi di contenuto si spostano con grande facilità fra piattaforme molto diverse, essendo visibili un anno su Orkut e il successivo su Facebook, un anno su BBM e il successivo su Twitter. Le piattaforme come Facebook hanno spesso cambiato esse stesse funzionalità, sviluppando o aggiungendo nuove caratteristiche.

Questo progetto di ricerca, dunque, non è uno studio delle piattaforme, ma uno studio di ciò che le persone postano e comunicano attraverso le piattaforme, del perché postiamo e delle conseguenze di queste attività. Abbiamo scoperto che questo contenuto varia sensibilmente nei nove campi in cui abbiamo lavorato. Il contenuto mostra e trasforma relazioni e argomenti locali. Il nostro studio pertanto ha riguardato sia il modo in cui il mondo ha cambiato i social media sia come i social media hanno cambiato il mondo.

Chiaramente non si tratta di un processo del tutto unidirezionale. Queste tecnologie hanno cambiato noi stessi. Ci hanno offerto un potenziale per comunicare e interagire che prima non avevamo. È necessario prima di tutto definire con chiarezza quali sono queste potenzialità e poi esaminare che cosa il mondo ha successivamente fatto con tali possibilità. È più facile capire che cosa sono i social media se andiamo indietro nel tempo quando non esistevano. Quindi riavvolgiamo il nastro attraverso Snapchat e Tinder, passati 
Facebook e QQ, attraverso MySpace e Friendster fino alla vita prima di tutto ciò.

Prima di tutte queste tecnologie, c'erano due modi principali mediante i quali le persone comunicavano tramite media. Un modo era quello dei media a diffusione pubblica, come la televisione, la radio e i giornali. Tali media consentivano pressoché a chiunque, a condizione che avesse ottenuto l'accesso a essi, di essere parte del pubblico. Le stazioni emittenti non hanno controllo diretto su chi costituisce il loro pubblico, benché possano cercare di convincere le persone a unirsi a loro. Disponibili da un bel po' di tempo sono anche i media che consentivano la comunicazione privata fra due persone nella forma di conversazioni uno-a-uno, per esempio una conversazione telefonica, definita anche comunicazione "diadica". Le persone si potevano incontrare in gruppi faccia a faccia, ma era insolito creare interazioni di gruppo dentro media come il telefono.

Con lo sviluppo di internet, questa polarizzazione tra media pubblici e privati ha iniziato a cambiare. Si poteva mandare una e-mail a un gruppo. C'erano i BBS (Bullettin Board Systems), i forum specializzati, le chat room e i blogging, che coinvolgevano pubblici più ampi, così come altri media di gruppo come le CB radio. Nondimeno, la maggior parte della comunicazione quotidiana attraverso i media rimaneva dominata dalle due forme principali, trasmissione pubblica e comunicazione diadica privata. L'iniziale sviluppo dei siti per la costruzione di reti sociali consistette, in effetti, in una graduale riduzione della trasmissione pubblica fino a diventare trasmissione di messaggi da parte di individui indirizzata a gruppi. Di solito tali gruppi includevano non più di poche centinaia di persone. Inoltre le persone che componevano questi gruppi interagivano fra loro, per esempio commentando i commenti degli altri.

Allo stesso tempo si consolidava lo sviluppo dei servizi di messaggistica e di internet tipo SMS e AOL. Questi si svilupparono ulteriormente con l'avvento dello smartphone, in particolare di Blackberry Messenger (BBM), la piattaforma di messaggistica brevettata per i telefoni Blackberry; il suo impatto globale è stato in genere notevolmente sottostimato e ha precorso WhatsApp. Tali servizi presero la messaggistica privata e la elevarono di livello includendovi varie funzioni di gruppo. Questa tendenza si è consolidata nel corso degli ultimi tre anni con il sorgere particolarmente repentino di piattaforme come WhatsApp $\mathrm{e}$ WeChat. Si tende a utilizzare queste ultime per creare gruppi più piccoli, più riservati rispetto a quelli di QQ o Facebook, di solito intorno alle 20 persone o anche meno. Inoltre possono non essere centrate su un qualsivoglia individuo, 
in genere tutti i partecipanti hanno la possibilità di postare allo stesso modo, si tratta di gruppi e non del network di una persona, quale che sia. Sono particolarmente importanti dato che per i giovani tale comunicazione testuale ha largamente sostituito l'uso vocale del telefono ${ }^{3}$.

Definire in modo chiaro che cosa sono i social media solo in base alle piattaforme che al momento presente esistono è limitante. Affinché la nostra definizione e il nostro approccio siano fondati dobbiamo considerare le nuove piattaforme di social media che vengono costantemente sviluppate, così come la probabilità che alcune diverranno quelle di maggior successo in futuro. È utile iniziare a scorgere un modello ricorrente nelle modalità in cui emergono nuove forme di social media.

Alcune di queste piattaforme sono derivate da una graduale riduzione della trasmissione pubblica, mentre altre da un graduale allargamento della comunicazione privata. Con l'aggiunta di nuove piattaforme in futuro, è probabile che si arrivi a un esito per cui di fatto si crea una certa gradualità tra il privato e il pubblico, lungo la quale si possono collocare queste piattaforme.

Possiamo individuare due scale chiave. La prima è la scala che va dal più privato al più pubblico. La seconda è la scala dal gruppo più ristretto al gruppo più largo. A un estremo di entrambe queste scale vediamo ancora la conversazione privata a due e all'altro estremo vediamo ancora la diffusione totalmente pubblica. Che cos'è che si va facendo graduale? Il nucleo dello studio delle scienze sociali è il modo in cui le persone si legano le une alle altre per formare relazioni sociali e società. Ciò si chiama socialità. Il miglior modo per definire quelli che sono comunemente chiamati social media, includendo anche i media precedenti, sta dunque nel descrivere la nuova situazione come una sempre più netta "socialità modulabile" ${ }^{4}$. 


\section{SCALABLE SOCIALITY}

HOW SOCIAL MEDIA HAS CREATED A NEW POTENTIAL FOR SOCIALITY

From the most private to the most public

From the smallest group to the largest group

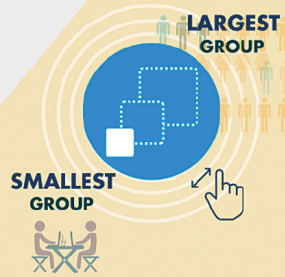

Fig. 1.1 Socialità modulabile

Uno dei più chiari esempi di come i social media hanno creato una socialità modulabile online è emerso dalla ricerca sul campo tra gli scolari di un paesino inglese. Basata su una inchiesta tra 2496 studenti, Miller ha scoperto che la maggior parte di loro era solita utilizzare cinque o sei diversi social media fin dalla tenera età (Fig. 1.1) (Fig. 1.2)

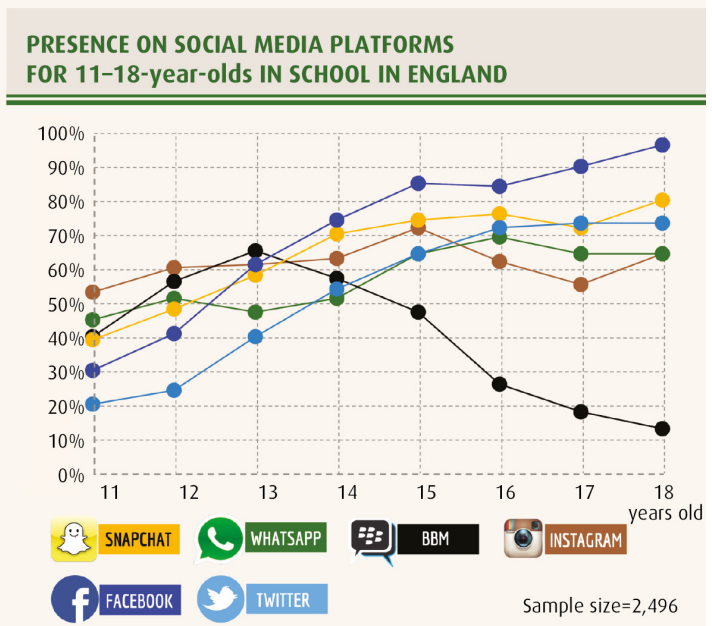

Fig. 1.2 Presenza sulle piattaforme di social media di ragazzi tra gli 11 e i 18 anni nelle scuole inglesi 
In un lavoro precedente, insieme a Madianou, Miller ha sviluppato un approccio detto "polimediale" , che riconosce che nessuna di queste piattaforme può essere correttamente compresa se considerata da sola, perché il significato e l'uso di ciascuna è in relazione alle altre. In precedenza si sarebbe potuto ipotizzare che il costo o l'accessibilità spiegassero perché gli utenti avessero scelto alcuni media o piattaforme piuttosto che altri. Oggi, tuttavia, le persone sembrano sempre più libere di scegliere fra queste piattaforme, e quindi si possono fare delle valutazioni sulla base delle loro scelte. Per esempio, i figli che vivono all'estero e che vogliono dire ai loro genitori con il dovuto garbo qualcosa che non farà loro piacere, come un nuovo tatuaggio o la necessità di più soldi, potrebbero per quel motivo evitare un medium che contenga un elemento visivo o uno che consenta a una persona di rispondere immediatamente - forse, dato il caso, arrabbiandosi.

Grazie alla polimedialità, le persone possono anche impostare differenti tipi di socialità in base alla diversità delle loro piattaforme di social media. Nel caso di questi studenti, possiamo mettere al primo posto la continuità della comunicazione a due, il messaggio o la telefonata per parlare in privato con qualcun altro come il loro Bestie o BBF (Best Friend Forever). Subito dopo viene Snapchat che, poiché spesso include elementi come fotografie particolarmente orribili del viso di qualcuno, si basa sulla fiducia. In effetti, può essere usato per stabilire e mantenere la fiducia entro un gruppo ristretto. Il passo successivo è fatto di gruppi creati su WhatsApp. Tipicamente, la classe scolastica avrà un gruppo di WhatsApp che include tutti i ragazzi che potranno parlare delle ragazze (o viceversa). Ce ne potrebbe essere un altro che include tutta la classe. I tweet sono in grado di raggiungere un gruppo ancora più largo, dato che arrivano a tutti coloro che seguono un individuo su Twitter. Questo è il principale sito delle prese in giro tra studenti, e può includere anche studenti di altre classi. Oltre la classe c'è Facebook, maggiormente usato da questi studenti per mantenere il loro legame con la famiglia, con i vicini e altri al di fuori della scuola. Infine abbiamo Instagram, dove ogni cerchia sociale di uno studente spesso è formata dagli studenti stessi della scuola. Tuttavia, è anche l'unico sito dove i ragazzi accolgono estranei, perché il contatto mostra che qualcuno che i ragazzi non conoscono ha apprezzato le qualità estetiche dell'immagine che hanno postato su Instagram (Fig. 1.3). 


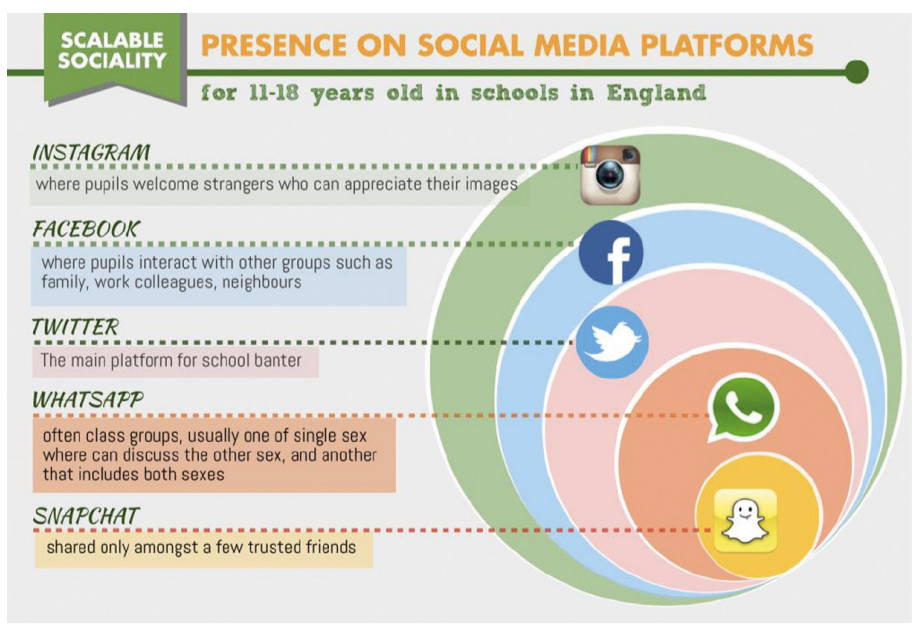

Fig. 1.3 Le scale di social media usate dagli scolari inglesi

L'uso dei social media da parte degli scolari inglesi dimostra la socialità modulabile nel fatto che ciascuna piattaforma corrisponde a una posizione di maggiore o minore privacy e a gruppi più stretti o più larghi. Non ci sono regole oltre queste. I gruppi e le piattaforme si possono sovrapporre, ma per lo più troviamo piattaforme legate a generi specifici di comunicazione che le persone ritengono adatte al gruppo coinvolto tramite quella particolare piattaforma.

Nel caso degli scolari, le differenze fra le piattaforme sono state usate per illustrare questo principio. In ogni caso, la socialità modulabile può esistere esattamente allo stesso modo dentro una singola piattaforma. Qualcuno può postare un commento o un'immagine sul proprio social media, ma questi post avranno senso soltanto per le persone che gli sono vicine, che capiscono a che cosa ci si riferisce. Altri possono stare nello stesso sito, ma non ne afferrano il significato - e per questo sono esclusi nonostante la loro presenza sulla stessa piattaforma ${ }^{6}$.

Abbiamo iniziato con questo esempio di socialità modulabile al fine di introdurre la questione della definizione di social media. Per quanto le definizioni possano essere utili, non sono in ogni caso l'obiettivo ultimo di questo progetto. Tramite le nostre dieci questioni chiave, ciascuna delle quali forma un capitolo di questo libro, esaminiamo la reale ampiezza dei contesti entro i quali i social media attualmente giocano un ruolo rilevante: dall'impor- 
tanza crescente della comunicazione visiva in opposizione a quella testuale fino all'impatto dei social sull'istruzione e se l'uguaglianza online influenzi la disuguaglianza offline.

Le piattaforme restano al centro della nostra analisi dato che queste sono le unità primarie attraverso le quali noi pensiamo e usiamo i social media. Eppure è necessario essere cauti nel presumere che ci siano proprietà delle piattaforme responsabili per le associazioni che osserviamo nelle piattaforme stesse, o che in un certo senso le causano. Gli studenti offrono prove del perché non dovremmo inferire cause semplicemente dall'associazione. In primo luogo il modo in cui usano Twitter, prevalentemente per prendersi in giro, contrasta nettamente con il modo in cui lo usano gli adulti, primariamente come una fonte di notizie. Qual è il "vero" Twitter, quello usato per informarsi o quello usato per prendersi in giro? Per di più, questo canzonarsi scolastico era solito svolgersi su BBM; poi si è spostato su Facebook e ora è quasi interamente su Twitter. Questo suggerisce che un genere di interazione può restare nettamente stabile anche se migra tra piattaforme che si suppongono molto diverse. Un tale esempio, qualora supportato da altri nei loro studi, suggerirebbe che la piattaforma è sorprendentemente irrilevante per spiegare perché e come le persone usano i social media. Essa fornisce il posto, ma non la causa né la spiegazione.

Nel momento in cui si considerano tutti e nove i campi di ricerca ci si rende conto subito che in ogni regione ogni nuova piattaforma per social media si standardizza velocemente su gruppi molto specifici di utenti e implicitamente se ne comprende l'uso appropriato e inappropriato, benché questi continueranno a cambiare. Di nuovo la differenziazione dei gruppi potrebbe accadere all'interno della stessa piattaforma o attraverso il contrasto fra piattaforme. Per esempio, nel 2014-15 c'era la tendenza in diversi dei nostri campi di ricerca a diversificare le piattaforme più visibili al pubblico come Facebook o QQ dalle piattaforme più private come WhatsApp/WeChat.

Il modo in cui nuove forme di social si dispongano sopra precedenti gruppi sociali, o invece creino nuovi gruppi varia considerevolmente da un campo all'altro. Nel nostro campo di ricerca in India del Sud, per esempio, i gruppi che si sono legati attorno ai social media sono per lo più unità sociali tradizionali come la famiglia o la casta. Al contrario, nel campo di ricerca nella Cina industriale la popolazione fluttuante di lavoratori migranti ha largamente perso la propria tradizionale forma di socialità, e in effetti crea gruppi sociali adatti a una nuova vita trascorsa spostandosi di città in città. 
La loro vita sociale più stabile è davvero sui social e non offline. In alcuni casi, le piattaforme più private hanno radicalmente cambiato le vite delle persone, come nel nostro campo di ricerca nella Turchia del Sudest in cui ragazze e ragazzi possono più facilmente conversare fra loro. Analogamente nel nostro campo in Cile la maggior parte delle persone usa postare pubblicamente a sostegno della propria comunità locale, di cui considera i valori in contrasto con quelli più metropolitani della capitale. Più dettagli su tutte queste affermazioni si troveranno nel seguito di questo libro.

Altri due punti inoltre dovrebbero essere chiari. All'inizio dello studio di internet si è comunemente parlato di due mondi: il virtuale e il reale. Oggi è decisamente evidente che tale distinzione non esiste - l'online è tanto reale esattamente quanto l'offline. I social media sono diventati già parte integrante della vita quotidiana a tal punto che non ha senso considerarli separati. Analogamente nessuno oggi riterrebbe una conversazione telefonica come qualcosa che si svolge in un mondo separato della "vita reale". È diventato evidente anche che la ricerca sui social non riguarda più il particolare ambito dei media o quello della comunicazione. La nostra ricerca presenta prove del fatto che i social media dovrebbero essere considerati piuttosto come un luogo dove molti di noi passano parte delle proprie vite. $\mathrm{Ne}$ consegue che quello dei social media è uno studio tanto della socialità quanto della comunicazione. Gran parte di questo libro non è sui media, e neanche sulla socialità modulabile. È sui social media come un altro posto in cui le persone vivono, accanto alla loro vita in ufficio, a casa, e nella comunità di appartenenza.

Se abbiamo qui definito l'espressione "social media", che dire di "mondo"? È chiaro che non possiamo studiare il mondo o il "globale" nella sua interezza. Ma possiamo ambire a studiare un numero sufficiente di contesti che ci consenta di produrre affermazioni sulla diversità e sulla generalità in tutto il mondo. I contenuti di questo libro sono basati sulle ricerche di nove antropologi, ciascuno dei quali ha trascorso 15 mesi osservando l'uso e le conseguenze dei social media in un particolare contesto. Il carattere unico di questo libro è che esso è quasi del tutto comparativo. Una breve sintesi dei nove campi di ricerca è disponibile in appendice ${ }^{7}$.

Infine, vorremmo collocare queste tematiche nei termini di un approccio sviluppato da Miller e Sinanan chiamato "teoria dell'acquisizione" 8 . Come vedremo in relazione ai dieci grandi temi considerati in questo volume, le nuove tecnologie sono spesso accompagnate da una sorta di panico morale, 
di frequente alimentato dal giornalismo, che spinge a pensare che la conseguenza di queste tecnologie sia la perdita di alcuni elementi essenziali della nostra autentica umanità. Tali paure, per esempio, includono l'idea che la comunicazione faccia a faccia sia più ricca o meno mediata della comunicazione che impiega le tecnologie digitali, o che stiamo perdendo capacità cognitive come la durata dell'attenzione a lungo termine. Queste reazioni alla tecnologia sono state comuni sin da quando Platone sostenne che l'invenzione della scrittura avrebbe danneggiato la nostra capacità di memoria. Allo stesso tempo altri hanno una visione utopistica secondo cui le nuove tecnologie ci renderebbero in una certa maniera post-umani.

La nostra teoria dell'acquisizione sostiene che queste tecnologie non creano differenze di qualsivoglia natura nella nostra essenziale umanità. Il sociologo Goffman ${ }^{9}$ ha mostrato in modo convincente che tutta la comunicazione e la socialità si collocano all'interno di generi culturali, inclusa la comunicazione faccia a faccia. Non esiste una cosa come la socialità o la comunicazione non mediata o pre- o non culturale. Al contrario, sarebbe importante riconoscere che qualsiasi cosa facciamo con le nuove tecnologie deve essere latente nella nostra umanità, ovvero qualcosa che, in quanto esseri umani, abbiamo sempre avuto la potenzialità di fare e di essere. Tale abilità è oggi acquisita come un risultato delle nuove tecnologie. Questa teoria non pretende di giudicare se ogni nuova capacità di inviare meme o selfie mediante i social, per esempio, sia buona o cattiva, ma soltanto di riconoscere che questo oggi è semplicemente diventato parte di ciò che gli esseri umani possono fare, come guidare un'automobile. 



\section{Capitolo 2 \\ Lo studio accademico dei social media}

Nell'introduzione a questo volume abbiamo definito i social media in termini di colonizzazione dello spazio compreso tra la trasmissione tradizionale e la comunicazione diadica privata, che fornisce alle persone una scala della misura del gruppo e del grado di riservatezza definita socialità modulabile. In ogni caso, non vorremmo che la nostra definizione venisse intesa in modo troppo rigido o assoluto. Ci sono stati molti casi precedenti di comunicazione di gruppo on line, come Bullettin Board System (BBS) e chat room. Sarebbe anche pedante ribadire che WhatsApp è una forma di social media quando è usata per un gruppo, ma non quando è usata solo fra due persone. La nostra definizione non è assoluta, non ha confini fissi; piuttosto si tratta di un dispositivo euristico che ci aiuta a chiarire i parametri del nostro studio, il cui ambito include siti come Tinder dove la comunicazione è diadica ma accessibile da parte di un gruppo, così come le console dei videogiochi, che oggi possono funzionare come piattaforme di social. $\grave{E}$ inoltre possibile prendere in considerazione anche alcuni blog e YouTube, ma qui essi appaiono raramente - anche perché su YouTube molto è postato nella forma di trasmissione pubblica, tanto da singoli individui quanto da aziende. Non si può neppure affermare che l'espressione "social media" sia derivata da una nostra scelta. Quando abbiamo iniziato la ricerca pensavamo che avremmo studiato "siti di social networking", ma abbiamo semplicemente seguito l'espressione colloquiale usata dal pubblico in genere. Quindi non si tratta di una categoria scientifica, ma di un'espressione comune sempre soggetta alle fluttuazioni della semantica pubblica, e noi possiamo essere responsabili solo del modo in cui la usiamo all'interno di questo libro.

La nostra definizione inoltre non sarebbe quella generalmente accolta. La pioniera riconosciuta nello studio dei social media è stata danah boyd ${ }^{1}$, e l'articolo più influente ${ }^{2}$ da segnalare è la pubblicazione del 2007 Social Network Sites: Definition, History, and Scholarship di boyd e Nicole Ellison ${ }^{3}$, in cui si presenta non solo una storia ma anche una sintesi dei lavori accademici prima del 2007. Come sottolinea boyd ${ }^{4}$, i primi siti di social networking negli USA si trovavano dove le persone potevano attivamente creare una rete dei contatti per trovare "amici di amici" o, come in siti del tipo 
Friends Reunited, per riconnettersi con amicis . Dopo un po' questi siti furono trasformati, in gran parte dai loro utenti, in luoghi di interazione più costante; questo si verificò di più fra amici consolidati, e così i siti riguardarono meno un networking espansivo. In un certo qual modo, pertanto, i siti di social networking diventarono social media, se dobbiamo prendere le categorie alla lettera.

Boyd fu anche una pioniera nel tentare di definire e caratterizzare i social media. Li descrisse come "networked publics" ("popolo della rete") e suggerì che possedessero quattro principali punti di forza: persistenza, visibilità, capacità di disseminazione, capacità di avviare ricerche ${ }^{6}$. I termini erano certamente di grande aiuto negli anni in cui queste idee iniziarono a essere sviluppate, sulla scia del grande successo di Friendster e poi di MySpace, seguiti da Facebook; questi concetti restano utili per comprendere tali piattaforme. Un recente libro su Twitter allarga il dibattito ai vari tipi di pubblico che sono collegati in rete e ai modi in cui gli utenti hanno sviluppato e diretto queste connessioni ${ }^{7}$.

Nel corso degli ultimi anni, tuttavia, e durante il nostro studio, la maggiore crescita ha riguardato le piattaforme - non solo WhatsApp e WeChat ${ }^{8}$, ma anche Instagram e Snapchat. Queste piattaforme non si allineano in modo particolare con i punti di forza delle precedenti. Non sono particolarmente votate alla ricerca, alla persistenza e nemmeno alla disseminazione, ma consolidano una tendenza verso la socialità modulabile. Forse non sorprende che, in quanto antropologi, saremmo a favore di una definizione che ponga l'accento sul tema della socialità, perché è ciò che studiamo. Altre discipline vedranno le cose diversamente. Per esempio, un libro recente sui social media scritto da uno studioso di comunicazione sviluppa un approccio basato sul concetto di connettività, e include parti su YouTube, Wikipedia e Flickr accanto a Twitter e Facebook ${ }^{9}$. È prevedibile che alle prospettive di discipline differenti si confacciano definizioni differenti.

C'è una naturale propensione a vedere le cose storicamente, assumendo che la tecnologia "evolva" secondo fasi ordinate e distinte. In quest'ottica, i social media appaiono essere nient'altro che l'ultimo uso popolare di internet, specie quando in alcuni paesi molte persone credono che Facebook e internet siano sinonimi. Oggi esiste un affermato filone di Internet Studies, che ovviamente vorrebbe assimilare lo studio dei social media al proprio interno ${ }^{10}$. Comunque sia, le discussioni relative a internet che si animarono precedentemente ai social media ${ }^{11}$ ebbero spesso un orientamento opposto 
rispetto a quello relativo ai dibattiti più recenti sui social media. Quando internet iniziò a svilupparsi, per esempio, la maggior parte lo considerava come un mondo virtuale separato là fuori, in grado di agevolare il convergere comune di gruppi con interessi specifici. C'era anche notevole preoccupazione riguardo a nuovi tipi di relazioni sociali rese possibili grazie all'anonimato $^{12}$. Al contrario, con riferimento ai social media, il tema dell'anonimato si è concentrato nel senso di una preoccupazione relativa alla perdita di privacy. Similmente l'enfasi su gruppi distinti con interessi specifici in rete ha riguardato anche una preoccupazione relativa al modo in cui reti sociali molto disparate di amici, familiari e professionali sono ora sovrapposte nello stesso spazio su Facebook. Invece di essere visti come un mondo "altro" virtuale, i social media sono accusati di essere implicati negli aspetti più banali del mondo di tutti i giorni, tipo 'unghia del piede dipinta' e 'cosa ho mangiato a colazione’. Ovviamente i social media si costruiscono sui precedenti usi di internet a fini sociali e comunicativi. Ancora, ci sono chiaramente sia molte discontinuità con i precedenti usi di internet sia continuità. Per questo sarebbe meglio garantire ai social media il loro status in quanto tema di ricerca, e essere aperti alla possibilità che spesso si tratti davvero dell'opposto di ciò che si riteneva fosse internet, anche se condividono lo stesso spazio. Per questo abbiamo in genere evitato un approccio storico che faccia dei social media il punto d'arrivo di una narrazione, considerata quindi come la causa di ciò che sono ora. Come indicato nell'introduzione, i social media non sono solo un allontanamento dallo studio dei precedenti usi di internet, ma sono anche più incorporati nella vita sociale di tutti i giorni. Come tali, offrono una nuova opportunità per far convergere gli studi sui media e sulla comunicazione nell'ambito di altre scienze sociali come l'antropologia.

danah boyd spicca come pioniera nello studio dei social media anche per aver organizzato una bibliografia online in cui tutti coloro che studiano i social possano inserire dettagliatamente le loro pubblicazioni ${ }^{13}$. Al momento della scrittura (aprile 2015) c'erano 669 voci. Queste rappresentano un'ampia varietà di discipline e di approcci. Nell'esaminarne alcune appare immediatamente che sono rappresentate diverse prospettive alquanto distinte, spesso con scarsa concimazione trasversale reciproca. Non intendiamo soffermarci su questo, tuttavia è importante notare che il grosso degli studi proviene da discipline che sono più dirette a metodi e prospettive influenzati dalle scienze naturali; dunque tirano in ballo casistiche di utenti, verifica di ipotesi e modelli. Qui non si affronta questo enorme corpus di materiali, 
perché il nucleo tematico di questo libro riguarda la comparazione fra diversi contesti culturali; i dati derivanti da una popolazione non possono essere estrapolati in relazione al comportamento di qualsiasi altra.

Il presente volume per questo va visto come complementare rispetto agli approcci dominanti allo studio dei social media e distinto da essi.

La bibliografia inoltre offre un motivo chiaro del perché nel presente volume ci siamo concentrati su un approccio comparativo. Gli utilizzatori dei siti Cinesi QQ (820 milioni), QZone (625 milioni) e WeChat (355 milioni) sommati sono certamente comparabili a quelli di Facebook (1.25 miliardi) ${ }^{14}$. QQ è anche più vecchio di Facebook. Eppure i titoli dei 668 studi elencati nella bibliografia di boyd non includono una sola menzione di QQ, QZone o WeChat, mentre ci sono 157 riferimenti a Facebook ${ }^{15}$. Anche prendendo in considerazione il tema linguistico ${ }^{16}$, ciò dimostra che il contatto con il materiale cinese per coloro che lavorano in inglese è limitato. Una tale discrepanza costituisce parte della legittimazione del nostro studio. La scienza naturale si basa in gran parte sullo studio di sostanze con proprietà universali e immutabili che consentono decontestualizzazioni generalizzate, e pertanto può non essere rilevante dove lo studio è stato condotto. Le scienze sociali tuttavia sono tutt'altra faccenda, e questo libro è un appello all'insegna della più elevata sensibilità per le differenze regionali e sociali e per le loro conseguenze.

Inoltre, c'è il rischio che gli studi basati sul modello delle scienze naturali possano moltiplicare analisi di piattaforme come Twitter perché il materiale è pubblico e disponibile, e quindi potenzialmente utilizzabile come ampio insieme di dati. È di gran lunga più difficile far uso di piattaforme come WhatsApp, dato che occorre conoscere alcune persone molto bene prima che accettino di darti fiducia e accesso alle loro conversazioni private. boyd conta una bibliografia addizionale di lavori su Twitter davvero estesa, eppure nessuno di questi siti ha un titolo che faccia riferimento a WhatsApp. Non è nostra intenzione disprezzare alcun approccio. Tutta la ricerca autentica offre documentazione valida. Se la nostra indicazione che alcuni metodi convenzionali producono categorie loro proprie e anche problemi di generalizzazione è fondata, allora è auspicabile che tali cautele siano condivise da coloro che si considerano coinvolti negli studi dei social media sul modello delle scienze naturali. 


\section{Storia e cultura}

Benché boyd e Ellison abbiano offerto una prospettiva realmente globale ${ }^{17}$, la bibliografia di boyd indica che tale prospettiva può non avere avuto seguito nel grosso della ricerca successiva, almeno in lingua inglese. Questo riguarda probabilmente anche la storia dei social media. In quanto antropologi la nostra prima attenzione non è verso l'invenzione della tecnologia o il lancio commerciale, ma verso le piattaforme che si affermarono in modo significativo entro particolari società. Avendo presente questo, la storia dei social media probabilmente inizia in Corea con il successo di Cyworld. La piattaforma venne lanciata nel 1999 e divenne la prima a ottenere una vera ubiquità tra i giovani appartenenti a una popolazione di un certo rilievo. Nel 2005 quasi tutti i giovani coreani usavano CYworld.

Una delle caratteristiche di Cyworld era che trattava le varie categorie di amicizia come serie di cerchi concentrici. Alcuni studiosi notarono allora che questo era analogo al modo in cui tradizionalmente operavano i sistemi di parentela in quella regione ${ }^{18}$. Se si accettava di essere un Cy-ilchon - un legame molto intimo - allora ci si legava socialmente secondo il principio di reciprocità, ad esempio commentandosi a vicenda in una maniera che ricordava queste relazioni di parentela. In breve ciò implicava uno specifico allineamento culturale fra la particolare società e la particolare piattaforma - possibile motivo del perché Cyworld ebbe tanto successo in Corea, e non altrove.

Il guaio è che è davvero difficile portare prove a sostegno di un tale argomento. Cyworld è stato massicciamente sostituito da Facebook in Corea. Eppure ciò può non avere nulla a che fare con la funzionalità né con l'allineamento culturale; potrebbe semplicemente essere un desiderio di seguire l'egemonia globale. Ci sono altri casi, in questo libro, di legame fra le caratteristiche delle piattaforme e le regioni con cui esse sono associate, maggiormente tramite esempi dalla Cina che ha proprie distinte piattaforme di social media. Dati i divieti politici di usare altre piattaforme in Cina, sembra tuttavia opportuna una certa cautela nell' interpretare queste associazioni culturali come causative.

Questo dilemma culturale del tipo l'uovo o la gallina è un problema comune nell'analisi dei social media. Per esempio, si sostiene spesso nel giornalismo e nella psicologia e sociologia popolare ${ }^{19}$ che i social media siano associati con l'individualismo e siano usati principalmente per il networking egocentrico o autocelebrativo. Gli approcci derivanti dagli studi critici ${ }^{20}$ 
vanno anche oltre, suggerendo che ciò si lega alla nostra politica economica contemporanea, che è essa stessa espressione di neo-liberismo ${ }^{21}$. Le aziende di social media sono, fra le altre cose, società capitaliste, e per questo propense a promuovere tali valori. In ogni caso, la gran parte di tali ricerche si svolge nel Nord America, un'area dove autori come De Tocqueville ${ }^{22}$ posero grande enfasi sull'individualismo come carattere culturale dominante in un periodo precedente al capitalismo, mentre la regione più di successo per il capitalismo per un lungo periodo della storia recente forse è stata la Scandinavia, un'area di solito rappresentata come relativamente collettivista in termini di valori. Benché ci si aspetterebbe che gli antropologi facessero luce su questioni culturali, proprio per questo noi intendiamo essere cauti sia riguardo al determinismo culturale sia riguardo al determinismo tecnologico.

Generalmente, sembra improbabile che un insieme di new media il cui uso primario era sviluppare e mantenere connessioni sociali sia meglio caratterizzato in termini di individualismo. In verità è possibile che alcune piattaforme di social media che all'inizio si svilupparono nel clima alquanto più individualista degli USA siano fiorite pienamente soltanto quando migrarono verso società più orientate al gruppo. Per esempio, una delle prime piattaforme più di successo fu Friendster. Questa si avviò negli Usa nel 2002, ma ottenne un più grande consenso in nazioni del Sudest asiatico, e alla fine venne acquistata da un imprenditore malese e reinsediata là. Analogamente, Orkut fu inizialmente sviluppato da Google, ma ottenne il suo primo impatto grazie all'altissima popolarità in nazioni come il Brasile e l'India; in seguito venne infatti reinsediata in Brasile. Oggi la piattaforma di social media più profondamente radicata in Cina è probabilmente QQ (fondata nel 1999, anche se inizialmente come un servizio di messaggistica istantanea $)^{23}$, che domina anche in termini di numero di utenti attivi ogni mese. Renren, una piattaforma Cinese molto simile a Facebook, è una componente più piccola del mondo di social media cinese ${ }^{24}$. QQ, come sarà chiaro, è una piattaforma più estesa, con più funzionalità e una maggiore integrazione nella vita delle persone rispetto a quanto emerso in una qualunque delle piattaforme di social media occidentali.

Più importante di qualsiasi allineamento alle propensioni culturali è stata la tendenza da parte di aree più periferiche a emulare quelle ritenute più metropolitane. Per questo motivo la storia dei social media, quella più standardizzata, non inizia con Cyworld, ma si focalizza su alcuni sviluppi 
negli Stati Uniti. Nel Nord America la prima piattaforma di social media a raggiungere questo grado di penetrazione sociale e di influenza fu MySpace (fondata nel 2003), che dal 2004 velocemente si consolidò con una vasta base di adolescenti. Col tempo si specializzò nel suo orientamento musicale. Altri siti specializzati come Friends Reunited (avviato nel 1999), usato come networking professionale e commerciale, mostrarono il potenziale per variazioni di nicchia - come fecero siti di appuntamenti come Grindr (fondato nel 2009), e ora Tinder (fondato nel 2012) ${ }^{25}$.

Fu probabilmente a causa del prestigio degli USA, insieme alla crescita della Silicon Valley come incubatrice di high-tech, che piattaforme come Friendster e Orkut, benché di maggior successo in altre parti del mondo, vennero all'inizio dagli Usa ${ }^{26}$. Proprio lo stesso fattore può ben aver portato alla loro fine, come quando, per un momento, Facebook apparve pronto a divorare i suoi fratelli e assumere il comando. A quel punto sembrò molto probabile che Facebook avrebbe affermato un dominio mondiale, se non fosse stato per l'intervento politico del governo cinese. Il nostro progetto dunque iniziò in un momento che sembrava fosse la fine della storia dei social media, ma nel corso degli ultimi due anni tale conclusione si è rivelata prematura. Al contrario, oggi vediamo svariati siti alternativi per i giovani, incluso Instagram (fondato nel 2010) e Snapchat (fondato nel 2011), mentre Facebook è cambiato diventando di maggior interesse per una base di utenti più matura, rilevante anche nella diffusione di Twitter (fondato nel 2006). Il dinamismo del settore è ben illustrato oggi dall'emergere significativamente repentino di siti come WeChat (fondato nel 2011) e WhatsApp (fondato nel 2009), Mentre Facebook ha 1.25 miliardi di utenti, WeChat ha raggiunto i 600 milioni e WhatsApp 800 milioni a ritmi molto più rapidi ${ }^{27}$.

Come prima accennato, sostenere che il successo di una piattaforma dipende in primo luogo dall'allineamento culturale è difficile tanto quanto sostenere che esso dipende dai suoi punti di forza dal punto di vista tecnologico. L'antropologia offre in ogni caso una spiegazione alternativa. In questo volume consideriamo l'allineamento culturale non come una causa, ma come una conseguenza. In altre parole, osserviamo il modo in cui una piattaforma si localizza, il che riflette inoltre il nostro maggior interesse per il contenuto piuttosto che per la piattaforma in $\mathrm{se}^{28}$. Una volta che si popola di contenuti locali, l'allineamento culturale segue come conseguenza.

Anche se una piattaforma può avere una forte identità in relazione all'azienda proprietaria, o anche una forte infrastruttura, è sempre soggetta alla 
trasformazione costante. Facebook è un buon esempio. Già nel 2007 boyd e Ellison indicarono che molte di queste piattaforme erano partite con funzioni e intenzioni decisamente differenti. Facebook è la piattaforma dell'inizio (con un altro nome) concepita per aiutare gli studenti maschi a fare una classifica delle studentesse, oppure è la piattaforma analizzata nel 2015? Per di più, i principali cambiamenti che si sono verificati, come la recente migrazione della piattaforma verso persone più grandi, possono essere in gran parte un risultato dell'attività degli utenti piuttosto che di qualcosa di voluto dall'azienda - proprio come siti precedenti come Friendster e Orkut ottennero seguito in regioni molto diverse da quelle per le quali erano state inventate.

Lo stesso punto sollevato a proposito delle trasformazioni nel tempo si applica alle differenze nello spazio, e questi sono i principali problemi per gli antropologi. Quando studiamo Facebook a Trinidad e scopriamo che differisce in modo significativo nel suo contenuto da Facebook in India, che cosa abbiamo scoperto? Se dicessimo che si tratta di uno studio del modo in cui gli abitanti di Trinidad si sono appropriati di Facebook, allora sembrerebbe che ci sia un Facebook più autentico collocato in qualche altro posto che ora è stato modificato dagli abitanti di Trinidad. Il punto messo in luce dal nostro approccio antropologico riguarda invece il fatto che Facebook esiste sempre e solo in relazione a una popolazione specifica; l'uso che ne fa un gruppo sociale non è più autentico di qualsiasi altro. Lo stesso problema emerge a proposito della parola 'trinidadiano' riguardo a Facebook. In una certa limitata misura, le persone di Trinidad sono state modificate dal loro uso di Facebook, ma esse sono sempre state in trasformazione. Il punto non è quindi come l'uno abbia modificato l'altro. Si dovrebbe invece riconoscere che noi studiamo la cultura, che è sempre dinamica in quanto esito del reciproco interagire delle persone e del mondo in cui vivono ${ }^{29}$.

Tali questioni stanno alla base del nostro studio comparativo, che deve porre in equilibrio prove di uniformità (persone e cose che diventano più simili) e di eterogeneità (persone e cose che differiscono maggiormente) a livello globale. In breve, nella misura in cui ognuno usa la stessa piattaforma potrebbe apparire che i social media siano uno strumento dell'omogeneizzazione globale. Eppure, nella misura in cui gli utenti rendono quella piattaforma qualcosa di specificamente locale, i social media sembrano essere uno strumento dell'eterogenizzazione globale ${ }^{30}$. Gran parte di questo volume riguarda l'irrobustimento di tale osservazione schematica. Prima, 
però, presentiamo una breve rassegna di alcuni dei principali problemi della letteratura accademica, a partire dagli studi su media e comunicazione fino alla sociologia e all'antropologia.

\section{Studi dedicati ai social media ${ }^{31}$}

Non abbiamo la pretesa di esaurire l'ampio numero di pubblicazioni e riviste specializzate che emerge dagli studi sui computer, su internet e sulla comunicazione e che, per questo, può definire lo studio sui social media come la prosecuzione più recente di ciò che era già stato stabilito all'interno della loro tradizione disciplinare. In particolare, come già indicato, questa panoramica non includerà ricerche condotte all'insegna di metodi che emulano le scienze naturali ${ }^{32}$. Il nostro interesse riguarda le considerazioni fatte sui social media basate sull'osservazione qualitativa del loro uso quotidiano. In ogni caso, anche solo questo lascia un'enorme quantità di ricerca.

Molte discussioni si sono sviluppate abbastanza precocemente. Un problema $^{33}$ ha riguardato il rapporto fra la socialità online e quella offline, insieme al riconoscimento che, a differenza dei modi precedenti di praticare internet, principalmente usato per contattare estranei con interessi comuni, i social media erano maggiormente focalizzati su relazioni sociali esistenti. Come boyd e Ellison notarono: "Ciò che rende i siti di social network unici non è il fatto che consentono agli individui di contattare sconosciuti, ma che permettono agli utenti di articolare e rendere visibili i loro network sociali" ${ }^{34}$. In parte per rispondere ai dibattiti pubblici che affermavano il contrario, tali studiosi insistettero sul fatto che gli utenti non confondono "amici" come termine di Facebook, per così dire, con l'amicizia offline. Il dibattito accademico inoltre cercava di dare conto del crescente timore comune riguardo ai social media come una minaccia per la privacy, specialmente fra gli utenti più giovani ${ }^{35}$. In riferimento alla diffusa preoccupazione sulla privacy e a quanto i ragazzini fossero consapevoli di ciò che stavano facendo, si aggiungevano le osservazioni sulle norme d'uso che sembrava stessero delineandosi. Gradualmente, una serie di riviste arrivò a dedicare sempre più spazio a tali studi sui social media. Una appropriata e attenta analisi di questi dibattiti richiederebbe di passare per i recenti temi di Computer in Human Behaviour, New Media \& Society, the Journal of Computer-Mediated Communication e i nuovi Social Media + Society, per non citarne che alcuni. 
Un altro ambito di discussione popolare si è articolato sui social media come risorsa usata dalle persone per presentare se stessi, fino alla questione del come tali persone online sono un riflesso o divergono dalle stesse persone offline. Altri argomenti sono stati il numero di amici o follower che poteva essere appropriato per ciascuna piattaforma, e il modo in cui ciò rifletteva i giudizi che le persone danno degli altri. Un argomento ulteriore, conseguente ai precedenti studi di internet, è stato se il networking crea una qualche sorta di comunità ${ }^{36}$. C'è anche un ovvio desiderio di esplorare come i social media impattano sul benessere delle persone - se, per esempio ${ }^{37} \mathrm{ci}$ rendono più felici o infelici. Trattandosi di questioni comuni e popolari, le abbiamo usate per strutturare questo libro mostrando come il nostro studio possa essere usato per rispondervi.

Queste discussioni e questi problemi non costituiscono le basi del nostro lavoro sul campo. Il grado in cui la nostra ricerca ha messo a fuoco un tema come l'attivismo politico non è fondato sui nostri interessi né sul suo ruolo entro gli attuali dibattiti accademici o popolari. È stato unicamente imposto dal grado di riscontro di questo tipo di attività fra gli informatori delle nostre etnografie. Sono i nostri informatori a decidere il nucleo della nostra ricerca durante l'etnografia. Da tale prospettiva il principale interesse di questa ricerca ha riguardato i social media nel contesto delle relazioni personali, dato che questa è solitamente la dimensione dominante nelle nostre esperienze etnografiche. Fortunatamente ci sono due libri molto chiari e utili che analizzano quest'ambito: uno di Baym ${ }^{38}$, riguardante i media più in generale, e l'altro di Chambers, con riferimento più specifico al tema dei social media ${ }^{39}$. Questi libri forniscono ampie bibliografie su argomenti come familiarità, incontri, amicizia e identità. Oltre a questo volume comparativo la presente serie editoriale include nove singole monografie, una per ciascun campo di ricerca, e tutte dedicano il loro capitolo 4 all'impatto dei social media sulle relazioni personali - riflettendo sul modo in cui queste sono dominate non solo riguardo all'uso ma anche alle conseguenze dei social media.

Probabilmente la gran parte degli studi sui social media nelle principali riviste si incentra su vari termini psicologici e sociologici, inclusi capitale sociale, gratificazione, benessere e status. In ogni caso, alcuni di questi sfumano nell'analisi correlativa, che tendiamo a evitare per via del livello di generalizzazione che solitamente tale analisi presuppone. Un tipico esempio di studi siffatti potrebbe essere quello di AL-Deen e Hendricks ${ }^{40}$, ma ce ne sono molti altri. 
Un'iniziativa rilevante è stata la fondazione del Pew Center for the Study of the Internet and American Life, nel 2004. Questo centro ha reso pubblico un flusso continuo di importanti inchieste e dibattiti approfonditi, fornendo la miglior guida disponibile sia in relazione all'estensione sia al tipo di utilizzo sia anche al senso delle opinioni degli utenti, almeno per come rilevata tramite questionari ${ }^{41}$. Di nuovo, tuttavia, la tendenza regionale sarà evidente sin dal titolo del progetto.

Una osservazione che emerge chiaramente da questa ricerca del Pew Center è la tendenza sempre crescente verso l'ubiquità. I teenager ormai guardano queste piattaforme e app ogni giorno, se non costantemente, e in genere sono attivi simultaneamente su più d'una, per lo più mediante l'accesso con telefono cellulare. Alcuni dei report del Pew riflettono la medesima tendenza emersa nel nostro progetto. La loro scoperta che la presenza femminile domina i siti più visivi, per esempio, è stata rilevata anche nel nostro studio degli studenti inglesi. Inoltre, come questo libro dimostrerà, le generalizzazioni si fanno più fragili quando si esaminano posti come la Turchia o la Cina. In effetti, alcuni degli studi del Pew sono utili anche perché mostrano tendenze degli USA molto specifiche, per esempio il grado in cui gli utenti americani condividono ogni settimana la loro religione online (Pwe 6/10/2014).

Ci sono molti approcci che intendono la loro prospettiva specifica soprattutto come studio critico. Essi proseguono una tradizione che risale alla cosiddetta Scuola di Francoforte ${ }^{42}$ di teoria sociale, e sono anche in primo piano in quelli che generalmente sono oggi definiti Studi culturali. Un chiaro esempio di questo tipo di prospettiva è il lavoro di Fuchs ${ }^{43}$, benché elementi del suo approccio si ritrovino anche in molti degli studi dominanti ${ }^{44}$. Gran parte di questo lavoro è rivolto alla critica delle aziende che possiedono i social media in quanto società capitaliste e prediligono argomenti che associano le conseguenze dei social media agli interessi di tali compagnie. L'altro grande tema di questi scritti è relativo all'uso statale di informazioni private, sulla scia delle rivelazioni di Wikileaks e di Edward Snowden.

All'altro capo dello spettro sta un numero un po' più grande di studi il cui principale interesse è aiutare le persone a fare soldi usando i social media o che sono incentrati sul valore delle azioni e sull'incremento delle società. Se gli studi critici tendono a presumere il male, gli studi commerciali tendono a presumere il bene ${ }^{45}$. I risultati del nostro progetto affrontano entrambi gli aspetti di questa equazione, includendo infatti sia come le persone guadagnano dai social media sia come le aziende di social media ne ricavano 
guadagni. Tuttavia, il nostro principale interesse sta nel delineare con grande attenzione le conseguenze dei modi in cui le persone usano i social, anche con la speranza di dar conto in modo attento del loro benessere avendo vissuto con ciascuna popolazione per un periodo prolungato.

Un altro importante approccio che offre un corpus notevole di prove dettagliate deriva dalla ricerca linguistica, che spesso include analisi molto accurate delle specificità del postare, come la natura della lingua e le norme d'uso. Un buon esempio di tale studio è quello di Lomborg ${ }^{46}$. Anche se il suo interesse principale è la teoria della comunicazione, egli mostra come emerge il genere culturale, attraverso uno studio dettagliato dell'uso dei social media come parte della vita quotidiana. Ciò significa riconoscere che i generi (ossia particolari stili d'uso) rappresentano un equilibrio fra vari fattori come intrattenimento, informazione e familiarità. Questo studio mostra come, anche in una piattaforma nata da poco, il contenuto riflette un'intera serie di generi differenti. Eppure i singoli utenti imparano molto rapidamente a negoziare tra questi spazi con grande facilità e agio. Interessanti per una prospettiva antropologica sarebbero inoltre studi che analizzassero la violazione delle norme $e^{47}$.

Via via che i social media sono divenuti più pervasivi, si è affermata la tendenza a studiare fenomeni specifici piuttosto che scrivere sulla natura dei social in se stessi. Oggi una rassegna esauriente della letteratura si ritroverebbe presto logora prima di riuscire a coprire l'impatto dei social media su religione, vita economica, istituzioni statali, pratica scientifica e così via. Il punto è che ci sono davvero pochi ambiti che si potrebbero non considerare inclusi nel titolo "l'impatto dei social media su..." ${ }^{4}$. Dato che questo volume include capitoli distinti su molti di questi argomenti, per esempio il genere, la disuguaglianza, politica, l'istruzione, l'ambito del visuale e il commercio, qui non si considera la letteratura su questi argomenti e interessi oggi molto disparati, che si può trovare invece all'interno dei capitoli dedicati. C'è una pletora di altri usi, per esempio nel campo sanitario, nel marketing o nei soccorsi in caso di calamità, che non sono trattati in alcun luogo in questo libro perché questo studio può parlare davvero soltanto di quello che è stato concretamente osservato nei nove campi di ricerca.

Invece di cercare di essere esaustivi, questa rassegna degli studi generali sui social media terminerà con un'indicazione sulle "best practices". Un'area si staglia come caso esemplare di qualità estremamente elevata e di reale ricerca al punto da costituire un modello da sviluppare in futuro. È il lavoro 
su come i social media (o, meglio, più in generale i nuovi media per la comunicazione) influenzano l'istruzione, e specialmente la più ampia nozione di apprendimento. Questi lavori hanno avuto la tendenza a essere multidisciplinari, inclusi alcuni di ambito antropologico. Si integrano, in modo complementare, lavori qualitativi, di tipo etnografico e a lungo termine, con panoramiche comparative a grande scala, a cavallo fra interessi decisamente accademici e rendiconti applicati e orientati al sociale.

Forse ciò riflette il fatto che gli accademici spesso hanno anche una certa esperienza di insegnamento. Queste pubblicazioni verranno discusse più in dettaglio nei capitoli sull'istruzione, e in ogni caso includono lavori di boyd, Clark, Ito e collaboratori e anche di Livingstone e collaboratori ${ }^{49}$. Per chi volesse avviare per proprio conto delle ricerche sull'uso e le conseguenze dei social media, questa è la letteratura che senza dubbio consiglieremmo. Auspichiamo che il nostro principale contributo risieda nella nostra prospettiva comparativa.

\section{Dagli studi di comunicazione agli studi sociali}

Sebbene gran parte dell'interesse iniziale sui social media sia scaturito dagli studi di comunicazione e di internet, complementare è stato il crescente interesse da parte degli studi sulla socialità, dal momento che si è arrivati a considerare i social media come un luogo entro cui le persone si associano tra loro. Ovviamente, la base disciplinare sarebbe la sociologia. Come indicato nel Capitolo 1, una figura fondamentale sia per la sociologia sia per lo studio della socialità, è stata George Simmel ${ }^{50}$, che ha discusso in modo ampio e sistematico il modo in cui le persone si legano l'una all'altra. La sociologia contemporanea è molto vasta, ma probabilmente i due autori più rilevanti nello studio di internet sono stati Castells e Wellman. Entrambi in modi differenti hanno messo a fuoco l'idea di network.

Non è possibile evitare alcuni tecnicismi nel considerare l'influente libro di Castells The Rise of the Network Society (trad. it. La nascita della società in rete, Università Bocconi Editore, 2014). Castells ${ }^{51}$ colloca la logica della nostra nuova politica economica entro forme globali di organizzazione, in cui il ruolo del "capitalismo informazionale" è sempre più critico. Egli descrive questo processo ponendo una distinzione sistematica fra la rete e il sé. Questi nuovi sistemi informativi creano forme innovative di potenti 
networks che si allineano ad altri al fine di sviluppare nuove forme di politica economica. Il network si dice che imponga una più elevata e implacabile "cultura della virtualità reale" sul secondo. Per questo, "noi non viviamo in un villaggio globale, ma in villette personalizzate prodotte globalmente e distribuite localmente" 52 . La nostra ricerca metterà in discussione queste generalizzazioni ad ampio raggio. Dalle prove presentate in questo volume, così come dal procedente lavoro degli antropologi ${ }^{53}$, non è affatto chiaro che l'uso locale dei social media e di internet sia in tal misura il prodotto di forze globali come la politica economica, e non invece di tratti culturali localizzati e molto specifici che identifichiamo nei generi più popolari della comunicazione.

Una simile enfasi sulla trasformazione radicale portata dalle tecnologie della comunicazione si trova nel recente libro di Rainie e Wellman Networked ${ }^{4}$. In modo molto ben argomentato, gli autori negano che la Social Network Revolution sia una terza rivoluzione, dopo la Internet Revolution e la Mobile Revolution. Gli autori esaminano una serie di cambiamenti sia nel modo in cui le persone creano network sia nella costituzione dei gruppi con i quali lo fanno. Si tratta di un libro del quale certamente consiglieremmo la lettura integrale a studenti interessati, benché il principale argomento degli autori sia uno di quelli che le nostre prove metterebbero in discussione. Gli autori vedono una tendenza continua attraverso la nascita di Internet, il telefono cellulare e oggi i social media, che porta a un declino delle relazioni fra persone e gruppi e la nascita del network basato sull'individuo. Presenteremo una sintesi delle nostre prove nel Capitolo 12. Ci sono casi in cui le prove supporterebbero gli argomenti di Rainie e Wellman. In certi casi di controllo tradizionale estremamente rigido, come nella Cina rurale o nel Sudest della Turchia, anche noi vediamo i social media nei termini di questo cambiamento che loro delineano in relazione ai network basati sull'individuo.

Ci sono tuttavia molte più situazioni nelle quali troviamo tracce che suggerirebbero una traiettoria diversa e persino opposta. Questo può essere in parte perché loro considerano i social media come una prosecuzione di tendenze che si sono sviluppate a partire da internet. Viceversa, noi riteniamo che in linea generale è meglio considerare i social media come un'inversione di queste tendenze. I social media segnano un ritorno al significato e alla vitalità di gruppi come la famiglia, la casta e la tribù, e un ripudio della precedente tendenza verso i network individuali. Per esempio, famiglie che sono state divise dalla migrazione o dal lavoro transnazionale usano i social media 
per colmare queste fratture ${ }^{55}$. Il concetto di socialità modulabile e l'avvio di piattaforme come WhatsApp chiaramente si riferiscono ai gruppi e non solo ai network individuali. Sarà inoltre evidente nel Capitolo 7 che molto dipende da fattori contestuali come lo status sociale e il genere, laddove l'online viene usato a volte per rafforzare e altre volte per completare elementi diversi della socialità offline ${ }^{56}$. Nello stesso tempo, Wellman e collaboratori ${ }^{57}$ hanno realizzato gran parte della ricerca sistematica dimostrando che i social media non sono un mondo virtuale. In particolare, è emerso che le relazioni online non andavano a discapito delle relazioni offline, se non per altro perché le persone che erano più connesse online avevano anche più connessioni offline. Sarebbe pertanto un fraintendimento ridurre questo raffinato approccio teorico un'unica tendenza verso il networking individuale.

\section{Il contributo dell'antropologia}

L'antropologia sarebbe in linea con le altre scienze sociali in virtù della sua lunga tradizione di studio sulla socialità umana e sulla comunicazione, ovvie basi per studiare i social media. Riguardo alla socialità, forse i contributi più noti dell'antropologia riguardano, da una parte, la parentela ${ }^{58}$ e dall'altra il dono, l'obbligo, lo scambio e la reciprocità in quanto aspetti costitutivi di una relazione sociale ${ }^{59}$. Consideriamo però la base dell'antropologia il termine "normativo", di solito non usato tanto di frequente ${ }^{60}$.

Che significa "normativo"? Riteniamo che le persone crescano sin dall'infanzia per essere socializzate in direzione di ciò che per il loro gruppo o società sarà considerato il comportamento appropriato o inappropriato. Quando un bambino fa qualcosa, un genitore può dire "non è questo il modo in cui ci si comporta" oppure "che cosa credi direbbe la gente se tu facessi questo in pubblico?". Con premi e punizioni “insegniamo" il comportamento appropriato. Gran parte si assimila solo mediante l'osservazione di ciò che coloro che ci circondano fanno o evitano di fare. Il motivo per cui le persone crescono e diventano "tipici" contadini del Kenya o nomadi siberiani non è genetico. Un/a bambino/a adottato/a alla nascita diverrà "tipico" della società dentro cui è stato/a socializzato/a, non di quella di nascita. Queste norme di comportamento appropriato o inappropriato non sono regole fisse; possono essere interpretate facendo ricorso all'immaginazione o anche derise. Nelle nostre etnografie tentiamo di presentare modi 
caratteristici in cui le persone a Trinidad, per esempio, usano i social media, ma anche di dare il senso dell'ambito più ampio e delle eccezioni che circondano tale uso, dato che nessuno è soltanto "tipicamente" trinidadiano. Così, gli antropologi studiano le norme non come delle regole, ma come l'analisi di ciò che le persone effettivamente fanno e del perché lo fanno, incluse le variazioni rispetto alle norme. In antropologia ciò è generalmente definito lo studio della "pratica" 61 .

Se la spina dorsale dell'antropologia è lo studio della normatività soprattutto entro le relazioni sociali, la disciplina ha anche molte membra, tra cui una fiorente antropologia dei media. Nel 2002, prima della diffusione dei social media, due volumi collettanei contribuirono a consolidare ciò che si era sviluppato come una consistente tradizione di media studies $^{62}$; oggi esistono diversi gruppi e associazioni dediti alla ricerca antropologica sui media ${ }^{63}$. Di recente sono anche stati fatti due tentativi di riassumere il contributo degli antropologi allo studio dei media digitali e più specificamente dei social media ${ }^{64}$. Una delle basi del nostro studio è stata la nascita nell' University College London del primo Master in Digital Anthropology, che diversi autori di questo libro hanno in precedenza frequentato da studenti.

Oltre ai media studies e alla socialità, forse la principale spinta verso uno studio antropologico specifico dei nuovi media è arrivata dall'importanza di questi ultimi per migranti e diasporici, spesso prodotti dai disastri politici o dalle esigenze della nostra moderna politica economica. Gli esempi includono una raccolta pubblicata sulle popolazioni indigene e la loro diaspora ${ }^{65}$, e la ricerca di Bernal ${ }^{66}$ sulla diaspora eritrea. Altre discipline hanno interessi simili, per esempio lo studio di Greschke dei migranti sudamericani diretti in Spagna ${ }^{67}$. Più di recente troviamo studi antropologici che guardano soprattutto all'impatto dei social media stessi su questi gruppi diasporici, che siano brasiliani, filippini, o uiguri ${ }^{68}$. Un piccolo ostacolo alla crescita di tale focus nell'ambito dello studio dei social media riguarda il fatto che esso può portare le persone a semplificare la precedente esperienza di comunità offline in opposizione a quelle online ${ }^{69}$. In realtà siamo sempre stati soggetti a varie forme di rottura.

Più in generale, per l'antropologia come per ogni altra disciplina, quando i social media sono divenuti ubiqui la ricerca si è frammentata in direzione di argomenti diversi e più specifici. Questi vanno dal lavoro di Coleman ${ }^{70}$ sui gruppi Anonimi e di Hacker fino al lavoro di Uilemon sull'arte studentesca e l'identità visiva in Tanzania ${ }^{71}$. Relativamente pochi antropologi 
hanno affrontato il tema della diversità culturale in sé ${ }^{72}$. C'è un'evidente sovrapposizione con gli studi sullo sviluppo, come in quelli di Boellstorff e altri ${ }^{73}$, e specialmente sui movimenti come ICT4D ${ }^{74}$, anch'essi focalizzati sull'impatto dei media ${ }^{75}$. Molti di questi approcci convergono attorno a un interesse per la diseguaglianza globale, che condividiamo con approcci sociologici e di altro tipo ${ }^{76}$. Particolarmente utile è stato il lavoro di Graham nell'ambito di una disciplina affine come la geografia umana, che è servito a illustrare graficamente la diseguaglianza in internet, nella comunicazione e nelle geografie dell'informazione ${ }^{77}$. Altri contributi mettono a fuoco temi della politica ${ }^{78}$ o del lavoro ${ }^{79}$. La principale caratteristica degli studi antropologici, comunque, è il suo concentrarsi su realtà locali ${ }^{80}$.

Curiosamente, dunque, è stato difficile giustificare una delle affermazioni più forti dell'antropologia, quella in favore degli studi comparativi. Nonostante le discussioni e le dichiarazioni a sostegno della comparazio$n \mathrm{e}^{81}$, e la tradizione di piccole comparazioni regionali su argomenti come la divinazione in Africa o il sistema dell'onore e vergogna nelle società del Mediterraneo, si tratta di una promessa quasi del tutto non mantenuta. Gli antropologi sono stati più a loro agio con il relativismo culturale ${ }^{82}$ e con la convinzione che ogni posto è unico, e più propensi a cedere le generalizzazioni, la teoria, ad altre scienze sociali. Questo si conferma come la sfida principale per la ricerca qui delineata. Saremo stati in grado, in qualche modo, di gestire il tipo di studio genuinamente comparativo che la disciplina ha talvolta richiamato come suo tratto veramente distintivo?

Il problema è che uno studio comparativo di molti siti dovrebbe non andare a discapito della profondità dell'impegno in ciascun singolo sito propria dell'etnografia. In effetti, per caratterizzare in modo adeguato la ricerca svolta in questo volume, l'enfasi andrebbe data tanto all'etnografia quanto all'antropologia. Il capitolo 3 presenta i lineamenti del nostro approccio etnografico e della nostra metodologia più in generale. Eppure gli antropologi non sono i soli che usano l'etnografia. Molti degli studi che sono discussi in questo capitolo derivano da un approccio qualitativo più ampio. Quelli che riteniamo da considerare maggiormente, come quello di Nancy Baym, danah boyd o Sonia Livingstone, usano un insieme di metodi che sono etnografici, basati sull'intervista, e anche quantitativi. La maggior differenza sta nel fatto che il nostro approccio, derivando specificamente dalla disciplina antropologica, ha una focalizzazione sullo studio comparativo della diversità culturale, che può comparire in questi altri studi ma che di solito ne rappre- 
senta un elemento marginale. Questo libro contiene anche un capitolo (il 4) sui nostri risultati quantitativi. Indica non solo perché il progetto ha accolto metodi quantitativi, ma anche perché i risultati sono stati trattati con molto più scetticismo rispetto a ogni altra prova in primo luogo qualitativa.

Lo scopo di questo capitolo non è sostenere che qualsiasi approccio può essere sufficiente per una comprensione completa dell'uso e delle conseguenze dei social media. Tutti gli approcci hanno i loro vantaggi e i commisurati svantaggi. L'antropologia offre profondità e comparazione, ma presenta notevoli problemi in relazione alla generalizzazione e tende a essere relativamente astorica. Se abbiamo messo in evidenza questo approccio è perché questo è un libro che presenta i risultati di un'indagine antropologica. Certo che, chiaramente, più le nostre scoperte potranno essere affiancate a studi storici, linguistici, di comunicazione e altri, maggiore sarà la comprensione che otterremo. 


\section{Capitolo 3 \\ Metodo e approccio}

\section{Essere capaci di fallire}

Se, in definitiva, questo progetto è considerato come pienamente riuscito, la ragione principale risiede paradossalmente nel fatto che ogni volta i nostri progetti individuali sono falliti. L'insuccesso è stato la componente principale dei nostri studi. Questi fallimenti rappresentano ciascuno la miglior prova a supporto dell'affermazione che in ciò che facciamo c'è coerenza e sapere. Affermando che i nostri progetti sono falliti, intendiamo semplicemente dire che essi non potevano realizzare determinati obiettivi di interesse accademico. Possiamo presentare quattro casi.

Costa ha svolto la sua ricerca di dottorato sul giornalismo on line, i digital media e i corrispondenti stranieri in Libano. Il suo interesse principale riguardava social media e politica. Un caso comparativo in Turchia avrebbe chiaramente favorito il suo percorso di ricerca. Scelse come terreno Mardin, un luogo non lontano dal confine siriano e diventata una zona curda autonoma. Mardin di per sé comprendeva una eterogenea popolazione di arabi, curdi, cristiani siriani e turchi. Era certo che questo avrebbe rappresentato il terreno ideale per i suoi studi di politica? Alla fine Costa nel suo libro scrisse un capitolo particolareggiato sulla politica ${ }^{1}$. La politica tuttavia divenne un elemento della sua indagine piuttosto che il focus principale, mentre l'importanza di genere e le relazioni familiari assunsero invece un ruolo centrale. Questo è dipeso dal peso assoluto di ogni impegno politico palese, coinvolgimento che in precedenza aveva causato la morte di molti parenti dei partecipanti, ragione per cui si evitava ampiamente di discutere di politica sui social. Facevano eccezione i sostenitori del governo o alcuni oppositori che si servivano di account falsi e anonimi. Nello stesso tempo, l'adozione di account multipli e falsi aveva facilitato una radicale trasformazione nella relazioni di genere, soprattutto nelle interazioni tra i giovani. Questo diventò effettivamente la narrazione principale. Costa nel suo approccio, piuttosto che concentrarsi sul tema che inizialmente aveva considerato più impor- 
tante e che poteva essere più utile per i suoi interessi personali o di carriera, seguì i materiali che aveva trovato.

Wang, al contrario, scelse il suo terreno di ricerca con cura perché intendeva studiare le relazioni familiari. Trovò un gruppo che si era formato sulla base di legami familiari molto stretti in cui tutto era vincolato su base parentale. Ora, però, i suoi membri avevano dovuto viaggiare per lavorare in città industriali diventando una popolazione fluttuante di migranti rurali, come si dice in Cina. Appariva pertanto certo che l'uso principale dei social avrebbe riguardato il mantenimento dei legami con le famiglie e i villaggi di provenienza dal momento che gli utenti tentavano di ricucire i legami interrotti dalla migrazione. Questo tuttavia non esaurì tutto ciò che Wang trovò. In realtà questi migranti semplicemente non usavano i social in questo modo e neppure sembrava volessero farlo. La principale ragione della migrazione ha a che fare con il fatto di vedere il mondo esterno e la giovane generazione di migranti dalle aree rurali usa i social media per tenersi lontana dal tradizionale controllo familiare e conquistare una certa autonomia come operai.

La situazione di Venkatraman fu persino peggiore. Scelse un terreno di ricerca nel sud dell'India che era stato costituito sino a un recente passato da villaggi rurali sparpagliati in un'area rurale. Successivamente, in quanto parte di ambiziosi progetti di sviluppo, vi furono ricollocate numerose compagnie informatiche e 200.000 lavoratori informatici. Il contesto appariva pertanto ideale, dal momento che presentava un netto contrasto tra quanto poteva essere considerato come la popolazione indiana più moderna accanto alla tradizione classica dell'ampio hinterland rurale. L'interesse principale di Venkatraman riguardava il rapporto tra social media e lavoro. Era certo che la base del suo libro sarebbe stato il contrasto tra questi due gruppi? Ogni cosa fu messa a punto perché fosse così.

Ebbene, leggendo il libro di Venkatraman questo contrasto si è in buona misura smorzato. C’è invece una certa enfasi sui caratteri specifici della società indiana tradizionale e sui modi in cui questi hanno avuto impatto sull'uso locale dei social, inclusi determinati fattori come il genere, la casta, la diseguaglianza e la famiglia estesa. Considerati come un insieme, questi fattori in realtà uniscono i lavoratori informatici e gli abitanti dei villaggi: i social media rivelano che quasi tutti rimangono 'indiani' del sud in modo caratteristico, uniti da quelle questioni di parentela e di casta nonostante l'estrema diversità delle circostanze. Tale giustapposizione certamente mette in rilievo la questione della gerarchia sociale, ma d'altro canto questo principio 
è sempre stato considerato dagli antropologi come centrale riguardo all'organizzazione della società dell'Asia meridionale. Così, ancora una volta, in misura notevole, non si è potuto seguire il piano iniziale sino in fondo dal momento che le evidenze hanno condotto a una diversa conclusione.

Il fallimento più spiacevole forse è stato quello di Haynes. Aveva accettato una posizione presso il Centro Interdisciplinare per gli Studi Indigeni e Interculturali nella Pontificia Università Cattolica di Santiago del Cile, sostenuta da una borsa del governo cileno espressamente destinata allo studio delle popolazioni indigene. Dal momento che il suo dottorato si era incentrato sugli indigeni urbanizzati di La Paz, in Bolivia, era stato naturale per Haynes spostarsi verso il Cile settentrionale che include un numero significativo di popolazioni aymara e quechua. Per lo studio dei social media, non volevamo però villaggi molto piccoli e i dati demografici dell'Alto Hospicio sembravano suggerire che quella area fosse di dimensioni adeguate con una ragionevole percentuale di persone indentificate come indigene, quanto meno per gli scopi del censimento (circa il 18\%). Il problema era che il suo studio sui social media mostrava con grande chiarezza che la maggior parte delle persone di origine indigena non si identificava attivamente come tale. Soltanto molto più avanti nella sua ricerca accadde che molti suoi amici intimi rivelarono la loro ascendenza indigena. In ultima analisi, il suo libro è molto istruttivo sul perché fosse così per la popolazione di quella città, in coerenza con una analoga soppressione di tutta una serie di altri potenziali aspetti di distinta identità. Pur nondimeno l'identità indigena non poteva proprio rappresentare il focus principale del suo studio sui social, anche se essa era stata la fonte del finanziamento.

Non tutto è andato storto. McDonald scelse un sito nell'hinterland cinese rurale, vicino al luogo di nascita di Confucio, e lì si è confrontato con quel livello di tradizione che era possibile prevedere. Sinanan aveva già lavorato a El Mirador, nelle Trinidad, dove vivono i suoi parenti, per cui non ci sono state particolari sorprese.

Che c'è dunque di così bello nel fallimento? Molti di questi antropologi avrebbe tratto un personale beneficio se le loro ricerche fossero andate come da programma, dal momento che i siti spesso erano scelti sulla base dei loro interessi accademici. Certamente avrebbero trascorso un minor numero di notti insonni. Tuttavia, è proprio qui che l'antropologia si differenzia da altri tipi di studio. Gli antropologi si sentono piuttosto frustrati quando redigono domande per ottenere finanziamenti che presupporrebbero che 
dalle loro ricerche si ottenessero risultati certi. La realtà è che spesso la vera ragione per cui antropologi come noi portano avanti le loro ricerche è che semplicemente non si sa a cosa si andrà incontro. La discrepanza tra ciò che programmiamo di conoscere e ciò che poi scopriamo è, per definizione, la parte principale della nostre ricerche originali. L'insuccesso, per noi almeno, è garanzia dell'integrità del nostro progetto. Per questo motivo crediamo che, alla fine dei 15 mesi di ricerca sul campo, ciascun ricercatore abbia accettato di doversi riorientare rispetto all'uso attuale e alle conseguenze dei social media. Pertanto, mentre per noi può trattarsi di una delusione, il lato positivo è che esso attesta la qualità e il successo della nostra scholarship. Ciò tuttavia significa che il nostro esame non si può leggere come il riflesso delle nostre opinioni. Quando sosteniamo che i social hanno questo o quell'impatto, non significa né che lo approviamo né che lo disapproviamo.

\section{L'etnografia è un metodo o un fine?}

In alcune discipline ${ }^{2}$, il termine etnografia è arrivato a significare le osservazioni dirette di ciò che le persone fanno nel loro habitat "naturale" piuttosto che, per esempio, ciò che esse dicono in risposta a questionari e test. Tuttavia, all'interno della disciplina antropologica un principio centrale dell'etnografia è il tempo. Una persona deve essere presente sul terreno di ricerca per un certo periodo, generalmente per più di un anno. Tutti i nostri ricercatori sono stati sul campo per almeno $15 \mathrm{mesi}^{3}$. Gli antropologi dovrebbero avere sufficiente competenza della lingua locale così da poter capire non solo le conversazioni che intrattengono con le persone del posto ma anche quelle delle persone tra di loro. L'etnografia in antropologia di solito viene descritta come "osservazione partecipante". Piuttosto che osservare a distanza con un quaderno di appunti, gli antropologi si lasciano coinvolgere: aiutano a badare ai bambini, servono da bere, vendono in un negozio di cellulari, stringono vere e proprie amicizie. Quando Miller si impegnò nella sua prima etnografia dello shopping ${ }^{4}$, i lettori erano sopresi di scoprire che spesso dava consigli ai suoi informatori su cosa comprare. Secondo lui il punto era che quando le persone vanno a fare compere con gli amici si aspettano che questi amici abbiano un'opinione su come sta un vestito o se sappiano di qualcosa di buono. È l'insuccesso nella partecipazione che rende il lavoro artificiale, non l'impegno a farlo. 
C'è un altro modo per definire l'etnografia che non si incentra tanto sul modo in cui ci si comporta, quanto piuttosto sul fine che si suppone di raggiungere. La questione cruciale dell'antropologia forse può essere meglio descritta come un impegno alla "contestualizzazione olistica". Nel momento in cui avviamo un tema di ricerca - nel nostro caso i social media - la nostra premessa è che semplicemente non sappiamo quale fattore della vita delle persone sarà influenzato da questo tema. Potrebbe essere il genere, la religione, il lavoro, la famiglia. Per di più, nessuno di questi ambiti può essere isolato per trarne delle correlazioni statistiche dal momento che nella vita reale essi non sono separati l'uno dall'altro. Una persona reale tutti i giorni della sua vita si confronta con questi e con una moltitudine di altri fattori contemporaneamente. Su Facebook un individuo può essere, nello stesso tempo, una donna, una hindu, una madre, un'operaria, un'introversa, un'amante delle soap opera, un'appassionata delle star di Bollywood. Non si tratta del fatto che in quanto antropologi cerchiamo di essere complicati e difficili; si tratta solo del fatto che crediamo che questo è il modo più verosimile per descrivere come le persone fanno esperienza del mondo. Nessuno vive all'interno di un tema di ricerca. La contestualizzazione olistica significa che tutto ciò che le persone fanno è il contesto per qualunque cosa facciano. In quanto metodo, l'etnografia non può realmente raggiungere ogni aspetto della vita di una persona, ma nel cercare di farlo si raggiunge almeno un senso più ampio di cosa possano significare questi aspetti.

Così, armati delle competenze linguistiche richieste e di tempo sufficiente, l'impegno consiste nel vivere in mezzo a un certo numero di persone diverse all'interno del campo di ricerca in modo che si possa partecipare e osservare quanto più pienamente possibile. Lo scopo specifico è che si comprenda abbastanza riguardo a tutti questi fattori - genere, religione, lavoro, tempo libero, famiglia - così da poter ragionare su cosa sia più o meno importante per capire come e perché le persone usano i social media nel modo in cui osserviamo farlo. Nella scienza, che ha a che fare con gli universali, un caso negativo può confutare una ipotesi. In una società, tuttavia, non c'è nulla che una singola persona faccia nello stesso modo e per la stessa ragione di un'altra. Un caso negativo è solo un caso in più. Il metodo non è aneddotico, perché cerchiamo comportamenti tipici costantemente ripetuti. Ma gli aneddoti, dunque le storie individuali, servono a trasmettere i nostri risultati. Spesso forniamo un esempio esteso in modo che si possa capire quanti fattori e profili contestuali sono rilevanti per uno specifico 
individuo. Spesso aggiungiamo anche altre storie più brevi per mostrare le molte varianti, nella misura in cui nessun individuo può rappresentare tutti i membri di una determinata società.

\section{Che cosa abbiamo fatto}

Perché questi nove siti? Perché non siti in Africa, nell'ex Unione Sovietica o nel sud est asiatico? Chiamiamo questo uno studio globale, intendendo dire che includiamo siti da tutto il mondo, cosa che ovviamente non significa che si tratta di uno studio comprensivo. Inizialmente c'era il desiderio di includere una popolazione quanto più ampia possibile e economie emergenti come il Brasile, la Cina, l'India. Non abbiamo mai avuto intenzione di lavorare in Nord America, semplicemente per il fatto che quest'area è già ampiamente sovrarappresentata negli studi sui social media. Al di là di questo, tuttavia, la selezione doveva dipendere dalle persone disponibili a condurre questo tipo di ricerca. Potevamo coinvolgere soltanto persone con una formazione antropologica che potessero anche impegnarsi in quel lasso di tempo. La proposta iniziale includeva uno studio in Africa, per esempio, ma la persona individuata non era disponibile. Pertanto, in definitiva, la logistica ha determinato i nove siti. Un altro fattore importante ha riguardato l'aspetto finanziario. Grazie alla generosità di un centro di ricerca finanziato dal governo, a Santiago, e della Wenner-Gren Foundation, abbiamo potuto includere altri due membri al gruppo finanziato generosamente dall'ERC, fornendo al progetto uno studio in Cile e un altro in Cina.

Ciascun antropologo è stato responsabile della selezione del sito attuale, tenuto conto del particolare interesse che intendeva coltivare, per esempio Costa per quanto riguarda la politica o Wang la migrazione. Una volta stabilito il sito di ricerca, bisognava lavorare con tutta la popolazione e non solo con una parte di essa: i più deboli e i più poveri, i religiosi e i secolari, i giovani e i vecchi, i maschi e le femmine. Per fortuna, molti di questi siti di ricerca hanno una forte tradizione di vita sociale aperta, così Nicolescu ha potuto passare del tempo in piazza, come gli italiani si aspettano si faccia, e Haynes ha potuto frequentare il mercato o le attività di doposcuola dei bambini, come avrebbero fatto i vicini. Quando ci sono comunità divise, i ricercatori hanno dovuto separare i gruppi di amici e le reti, come ha fatto Costa con gli arabi e i curdi a Mardin. Tuttavia, neppure questo è stato 
sufficiente. Dal momento che non esistono curdi 'tipici', Costa ha dovuto assicurarsi di aver inserito amicizie con famiglie curde raffinate e ben istruite in cui gli uomini lavoravano nel settore pubblico, e famiglie curde poco istruite e non raffinate, arrivate di recente in città migrando da fattorie. Wang ha dovuto assicurarsi di mantenere buone relazioni con i proprietari delle fabbriche di cui studiava i lavoratori, cosa che significava essere discreti e prudenti. In alcune occasioni, le ricercatrici hanno coinvolto assistenti maschi locali e i ricercatori assistenti donne per assicurarsi un migliore accesso.

Forse il sito di ricerca più difficile è stato l'Inghilterra, perché gli inglesi possono non essere particolarmente socievoli con gli stranieri e possono persino non conoscere il nome dei loro vicini. Molte persone che vivono in piccoli centri sono pendolari e non partecipano agli eventi sociali del posto. Se si frequentano semplicemente gli eventi 'comunitari' si potrebbe incontrare soltanto la frazione di gente locale che condivide l'idea di comunità. Pertanto, per i primi sei mesi Miller ha bussato di porta in porta ${ }^{5}$ cercando di convincere le persone a lasciarlo entrare nelle loro case. Soltanto così poteva essere certo di includere persone anti-sociali o sole e altri non necessariamente aperti al senso di comunità. Miller ha lavorato anche con le scuole dal momento che tutti vanno a scuola, e con gli ospizi, dal momento che tutti prima o poi moriranno.

La partecipazione significa proprio questo. Mentre McDonald faceva ricerca, era evidente che i bambini gridavano con allegria nel momento in cui l'antropologo si presentava, mentre gli adulti facevano grandi sorrisi. Sembrava davvero che fosse il miglior amico di tutti, ma in parte lo era perché viveva in quartieri estremamente ordinari, condividendo con altre famiglie il servizio igienico esterno e combattendo con l'erogazione irregolare di acqua e luce come facevano gli altri. Per un accademico come Wang trascorrere 15 mesi con operai di industrie i cui interessi primari, dopo una dura giornata di lavoro, consistevano nel giocare continuamente a giochi ripetitivi, e che spesso erano troppo stanchi per dire qualcosa di particolarmente sensato, è stato (per essere onesti) molto noioso. Nel villaggio in cui ha vissuto Spyer il gossip sembrava circolare senza condizioni e in effetti era diventato la principale forma di intrattenimento. Senza sorpresa si speculava anche su chi potesse essere 'realmente' questo intruso. Si erano diffuse voci che lavorasse per la CIA. All'inizio non era troppo problematico, ma quando le persone cominciarono a supporre che stesse investigando sui signori locali della droga, Spyer cominciò a preoccuparsi, dal momento che in città 
molte persone era state uccise a causa del traffico di droga. La situazione divenne più seria quando cominciarono a manifestarsi minacce personali. Fortunatamente l'arrivo della moglie sembrò acquietare le paure della gente del posto.

Haynes ha fatto l'esperienza di un punto di svolta della propria ricerca quando un terremoto di magnitudo 8.3 della scala Richter distrusse gli edifici di Alto Hospicio. Mancarono acqua ed elettricità per più di una settimana e l'autostrada che portava alla citta più grande di Iquique era crollata per il terremoto e la città pertanto era tagliata fuori. Questo determinò una certa coesione nella comunità dando a Haynes un nuovo senso di essere parte del tessuto sociale della città e anche momenti di unione persino un anno dopo. Nella sua ultima settimana di ricerca sul campo, persino con due nuove conoscenze, quando il terremoto fu argomento di conversazione, tutti e tre ammisero con un sorriso di aver pensato che sarebbero morti e ne nacque un'amicizia più profonda.

Un aspetto importante dell'osservazione partecipante è quello di imparare quale sia il comportamento ritenuto adeguato. Un adattamento graduale al modo in cui, in ciascuno dei siti, la gente fa amicizia è in questo senso parte integrante di questo metodo. A Trinidad fare amicizia ed essere amici con un gruppo di persone richiede festeggiamenti continui; per un gruppo diverso che vive nella stessa area, si diventa amici partecipando assiduamente alle riunioni religiose o alle cerimonie del ciclo di vita. Un etnografo deve essere camaleontico, capace di cambiare i propri modi di fare, la propria apparenza e il proprio linguaggio in relazione ai diversi gruppi, per far sì che ciascuno sia a proprio agio quando lui è presente.

Un ambito importante in cui generalmente falliamo, e perciò impariamo, riguarda la propria apparenza. Venkatraman può pure essere un indiano della regione in cui ha svolto la sua ricerca, ma ha avuto anche lui una lezione da imparare. Quando iniziò la sua ricerca nel sud dell'India indossava maglietta e jeans (fig. 3.1). Praticamente nessuno parlava con lui. Quando provò ad andare all'estremo opposto indossando pantaloni e camicia formali si rese conto che molta gente pensava che stesse provando a vendere qualcosa perché sembrava un uomo d'affari. In un caso, dopo aver spiegato con pazienza in una scuola la natura del progetto e la ricerca che avrebbe condotto, l'insegnante si scusò ma disse con fermezza che la scuola non era interessata ad acquistare quell'antropologia'. Alla fine Venkatraman trovò altre soluzioni. Si fece il piercing in entrambe le orecchie e cominciò 
a indossare i kurta indiani tessuti a mano, abbigliamento considerato 'da intellettuale', per posizionarsi in modo chiaro come un accademico. Dopo questo, la ricerca andò bene.

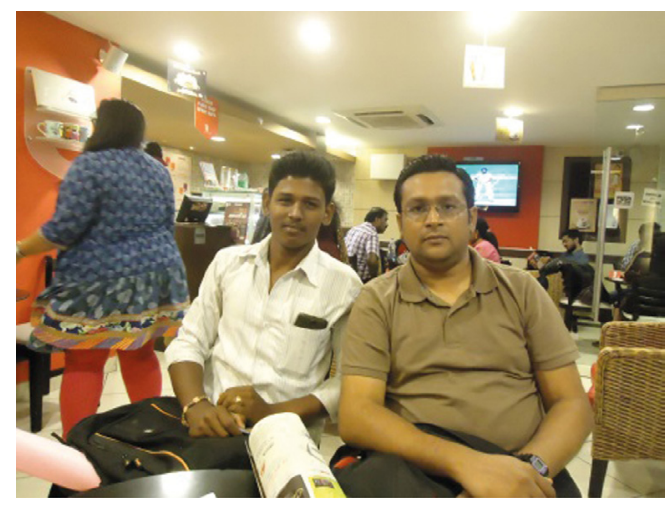

Fig. 3.1 Venkatraman vestito in modo inappropriato

Spyer trovò che il suo sito era spaccato tra cristiani evangelici e altri interessati ad alcune attività molto non cristiane. Perciò aveva bisogno di apparire né come una 'persona di Dio' né come una 'persona del mondo'. In alternativa optò per uno stile europeo che gli dava un aspetto neutro di 'gringo', consentendogli di parlare con le persone di entrambi i gruppi. Sinanan, invece, dovette mettere da canto la gran parte dei suoi vestiti e adottare un costume di Carnevale (fig. 3.2) per riuscire ad avere un rapporto con persone che stavano lavorando alla organizzazione di un campeggio di Carnevale. Costa capì che doveva depilare le gambe e le ascelle con maggiore attenzione del solito perché in quella parte della Turchia mostrare un singolo pelo quando le gambe o le braccia non sono coperte era considerato vergognoso. Capì pure che doveva tenere la casa immacolata e che talvolta doveva indossare un velo (fig. 3.3). 


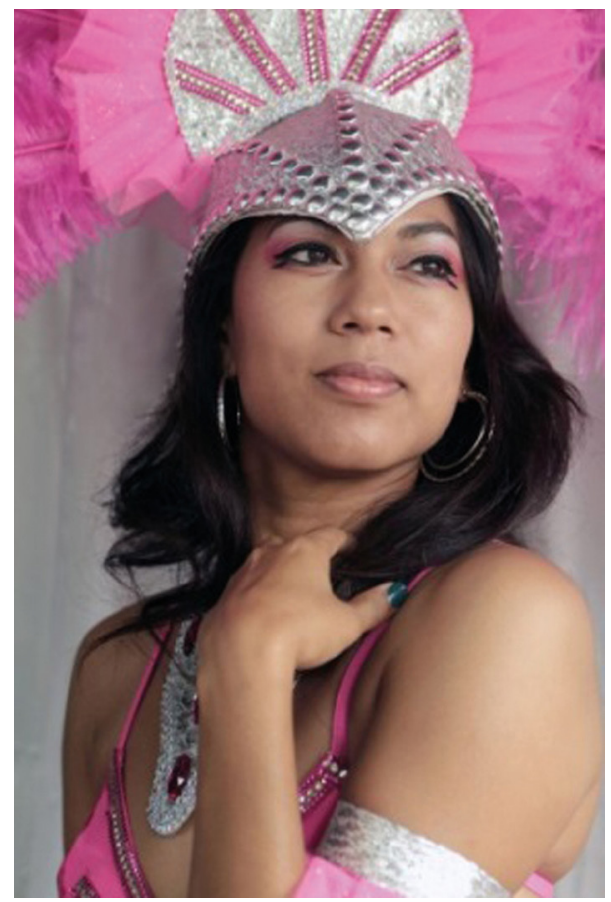

Fig. 3.2 Sinanan senza velo

Anche quando avevamo questionari strutturati o c'era accordo sullo studio di una particolare questione, spesso risultava semplicemente troppo artificiale porre la questione direttamente. I lavoratori dell'industria di Wang erano piuttosto sospettosi sulle domande dirette; essi avrebbero solo parlato con lei se fosse stata un'amica che li aiutava a trattare gli aspetti emozionali delle loro relazioni. Queste, per esempio, erano tre domande formali dell'indagine:

- Conosci la password dei tuoi partner sui social media e, se sì, mi potresti dire perché e come mai la conosci?

- Generalmente togli le foto dei tuoi ex partner dal tuo profilo? Se sì, perché? Secondo te, perché alcuni mantengono le foto dei loro ex partner sui social?

- Come gestisci le foto dei partner dei tuoi ex partner sui social? 


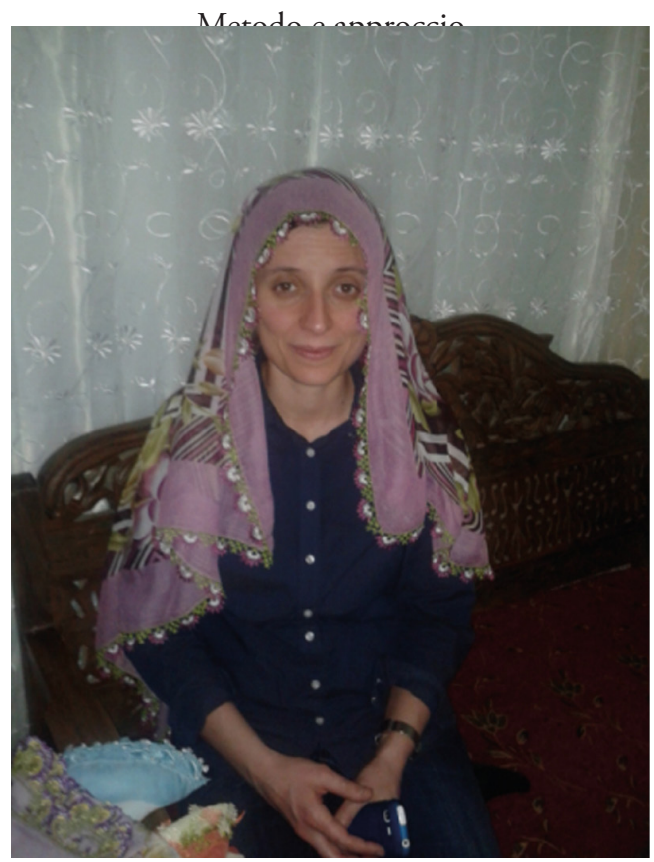

Fig. 3.3. Elisabetta Costa mentre indossa il velo

In pratica, questo il tipo di risposte a questo genere di domande:

- 'Oh, mamma! Ma come? È del tutto scandaloso, non ci posso credo credere. Ma ... sei grande, com'è che hai la sua password? Il mio ragazzo non me l'ha mai detta!'

- 'Calma, sei forte, e spero che impari la lezione. Ad ogni modo, gli hai dato qualche avvertimento o almeno un suggerimento?'

- 'Davvero?! E così... significa che sapeva che guardavi queste foto? Non lo capisco, cosa c'è che non va negli uomini? Perché pensano che possiamo accettare queste ex-puttane ... con una gran sorriso?! Proprio non lo capisco!'

Il punto è che le persone devono sentirsi a loro agio con l'antropologo/a e in teoria devono compiacersi del loro impegno con lui/lei. Diversamente, non ci dedicheranno il loro tempo. Inoltre, tutti i lavori di campo prevedono interviste registrate, realizzate soprattutto dopo che i ricercatori hanno costruito relazioni di fiducia. Questo ci consente di includere nei libri le parole esatte dei nostri informatori.

È impossibile indicare un campione di partecipanti preciso - questa è una domanda che ci fanno spesso. Talvolta si tratta di una conversazione casuale 
mentre si beve qualcosa, talaltra si tratta di persone che abbiamo visto ogni giorno per mesi. Non deve sorprendere il fatto che gli etnografi tendono a dare un quadro più profondo con quelli con cui sono amici da 10-15 mesi. Questi amici possono ammettere di aver detto inizialmente certe cose per impressionare o per nascondersi. Può accadere che in seguito essi diano una visione più ricca di ciò che pensano realmente delle cose che accadono intorno a loro. Gli antropologi valutano costantemente la validità di quanto ascoltano, aperti, ma nello stesso tempo scettici sulle dicerie. In qualche modo come un detective, dobbiamo sempre cercare di verificare le nostre fonti, sempre alla ricerca di ulteriori o migliori prove.

Da un certo punto di vista questo studio presenta un preciso vantaggio rispetto ad altri resoconti etnografici, che deriva dal soggetto dei social media. Prima di iniziare l'etnografia abbiamo concordato che tutti i ricercatori seguissero circa 150 persone on line per l'intera durata della ricerca. In qualunque modo ci si integri all'interno di una comunità, un/a antropologo/a sarà preoccupato/a di quanto la sua presenza potrà alterare il comportamento delle persone. Sembrava chiaro tuttavia che essendo una persona tra centinaia di altre su Facebook o QQ, l'impatto sul comportamento della gente on line fosse verosimilmente limitato; in questo senso postare on line sembra un esempio ideale delle possibilità di osservazione partecipante diretta rispetto al tema del nostro progetto. A questo scopo, ogni ricercatore ha creato un unico account sulle principali piattaforme di social. Ci sono state alcune discussioni circa il fatto se la presenza dovesse essere passiva o attiva. Sinanan ha scoperto molto presto che la gente si sentiva a proprio agio solo se l'antropologo era attivo almeno quanto un 'amico' lo sarebbe stato, esprimendo di tanto in tanto un "mi piace" e postando di sua iniziativa.

L'altra componente della nostra ricerca sul campo era il nostro questionario, più sistematico, discusso nel Capitolo 4. Come viene sottolineato lì, il nostro primo questionario era inteso come un tentativo di esplorazione che alcuni di noi erano soliti fare per cercare di garantirsi l'impegno con l'intero spettro della popolazione; questo questionario era infatti organizzato secondo la stima del reddito dei partecipanti oppure, in alcuni casi, della casta, del livello di istruzione o della religione. Il secondo questionario è stato realizzato alla fine della ricerca ed è più affidabile dal punto di vista della comparazione, ragion per cui esso costituisce la base di quel capitolo. Ha certamente aiutato il fatto che Venkatraman sia uno statistico di professione che è riuscito pertanto a organizzare e ad analizzare molto del nostro mate- 
riale. Una quantità consistente derivava dal semplice conteggio di ciò che le persone fanno sui social, $\mathrm{o}-$ in un caso - calcolando con chi esse interagivano per poi scoprire attraverso la discussione chi fossero realmente queste persone. Questo materiale tuttavia non è ancora stato analizzato ${ }^{6}$.

Durante questo primo anno di ricerca sul campo abbiamo capito che, anche se investivamo molto tempo nella condivisione di resoconti mensili, quando qualcuno poteva effettivamente visitare il sito di ricerca di qualcun altro risultavano evidenti molte più cose. Questo era un problema dal momento che, sfortunatamente, tu - lettore - non puoi visitare tutti i siti. Abbiamo pertanto stabilito che, oltre a scrivere, ognuno si sarebbe impegnato a realizzare 10 brevi video. Uno poteva riguardare il proprio sito e un altro il modo in cui era stato fatto il lavoro, il resto con storie o casi che illustravano cosa il ricercatore aveva trovato. Molti di questi, come quelli relativi al sud dell'India e al nord del Cile, sono stati realizzati da altri che generosamente avevano deciso di collaborare con noi ${ }^{7}$. Tutti questi film stanno su YouTube e sul nostro sito Why We $\mathrm{Post}^{8}$. Se si vogliono apprezzare pienamente le nostre testimonianze, suggeriamo di 'visitare' i siti attraverso questi filmati.

Questa discussione in quanto descrizione del metodo etnografico resta breve. Dare maggiori dettagli avrebbe compromesso l'intenzione di questa pubblicazione di raggiungere anche lettori non accademici. Per fortuna un recente libro fornisce un'eccellente introduzione a questa materia riguardo a pianificazione e conduzione del fieldwork con tutte le diverse considerazioni connesse, dall'etica all'archiviazione dei dati'. Anche se si riferisce agli studi on line, cosa che rende il contesto in qualche modo diverso rispetto al nostro approccio a un contestualizzazione di tipo olistico, così come prima descritta, la discussione sull'etnografia in sé, in termini di argomenti, progetto di ricerca, osservazione e interviste, può riferirsi a entrambi gli approcci.

\section{Lavoro comparativo e collaborativo}

La maggior parte del lavoro antropologico e etnografico è altamente individualistico, trattandosi di una singola persona isolata nel proprio campo. Questo progetto pertanto è inusuale, persino all'interno dell'antropologia, nel suo impegno sia nel lavoro comparativo che in quello collaborativo. Forse è così perché si tratta di un lavoro senza precedenti, dal momento che 
è solo recentemente che la comunicazione digitale a basso costo ha reso più facile stare in contatto durante una ricerca sul campo. La maggior parte del gruppo si è costituita come tale a settembre 2012. Dopo la preparazione, siamo andati tutti sul campo ad aprile 2013, con l'eccezione di Haynes che ha cominciato dopo. Per ogni mese di fieldwork lavoravamo tutti sullo stesso tema e poi facevamo circolare 5000 parole sul soggetto e ne discutevamo in video conferenza. All'inizio seguivamo un piano: il primo mese descrivevamo il sito, il secondo discutevamo del panorama dei social. Via via che passava il tempo, ci adattavamo agli imprevisti che trovavamo. Per esempio, avevamo stabilito di lavorare di più sulla morte e i processi di memorializzazione, ma molti siti di social media avevano relativamente poche persone anziane e questo fatto rendeva inutile stare un mese a osservare e a scrivere su questo tema. D'altro canto, un tema quale l'educazione, che in origine non avevamo programmato tra i nostri soggetti, cominciò chiaramente a diventare importante in tutti i siti, per cui vi abbiamo dedicato un mese. Sin dall'inizio eravamo d'accordo che ognuno avesse anche un tema individuale perché, ovviamente, potevano presentarsi argomenti di notevole importanza in un sito piuttosto che in un altro. Spyer, per esempio, avrebbe lavorato sulla mobilità sociale, Sinanan sul visuale.

Si potrebbe pensare che lavorare sugli stessi temi conduca a risultati simili e a ripetizioni. Abbiamo invece constato il contrario. Durante la ricerca siamo stati sempre consapevoli di quanto ogni sito fosse diverso. Di norma, quando si fa un' etnografia, all'inizio tutto sembra strano e bisognevole di spiegazione. Dopo pochi mesi, tuttavia, si corre il rischio di dare le cose per scontate e esse diventano ovvie, per cui dimentichiamo che dobbiamo scriverne per spiegarle agli altri. Per questo progetto, il fatto di leggere reciprocamente il lavoro ci ricordava le differenze che bisognava spiegare. Talvolta c'erano comparazioni regionali: il dualismo del Pentecostalismo rispetto agli inferi, nel sito di Spyer, sembrava una versione estrema del dualismo del Carnevale contro i valori del Natale a Trinidad, forse perché entrambi i siti hanno una storia comune in termini di schiavitù e conversione religiosa. Riguardo al sito di McDonald ci si aspettava che mostrasse sia somiglianze sia differenze rispetto a quello di Wang. Il primo rappresentava la stabilità di lungo termine in fatto di valori e forma di residenza, quello di Wang la rottura del trasferimento alle industrie. Tuttavia c'erano ancora soprese: per esempio, la differenza estrema di atteggiamento verso l'educazione, di importanza significativa nel sito rurale e scarsamente importante nel sito industriale. 
Ancora una volta abbiamo imparato di non poter generalizzare da un singolo sito. Sinanan ha scoperto il concetto di "Facebook Fame" in relazione al suo sito di Trinidad in cui le persone avrebbero fatto qualunque cosa pur di diventare virali (per esempio, i combattimenti tra gli scolari), mentre Haynes, riguardo al suo sito del Cile del Nord, rilevava che le persone non avevano il minimo desiderio di quel tipo di fama; avrebbero provato imbarazzo per quel tipo di attenzione. Questo grado di variazione è più chiaramente espresso nella nostra lista di scoperte sul sito Why We Post (www. ucl.ac.uk/why-we-post). In ogni caso, le nostre principali generalizzazioni e domande sono compensate da nove commenti per ciascuno dei siti. Se si leggono, si vedrà che quasi sempre almeno uno dei siti è del tutto in disaccordo con specifiche generalizzazioni. Questo tipo di risultato è evidente anche nella nostre risposte alle dieci domande che costituiscono la gran parte di questo libro. Troveremo, per esempio, che in molti casi i social media non rappresentano un aumento dell'individualismo, anche se in pochi casi è esattamente questo che rappresentano.

Il più delle volte potremo vedere maggiori similarità se guardiamo a generi tipo memi, selfies, post indiretti ${ }^{10}$ e così via, ma troveremo maggiori differenze se chiediamo cosa significhino esattamente, in ogni sito, termini quali privacy, amicizia, selfies. La comune lingua inglese può esagerare il grado di similarità. Per esempio, anche se il termine 'amico' ricorre in tutto il libro, fa ovviamnete riferimento a termini diversi con diversi significati. Nel sito brasiliano il termine 'colegas' descrive le relazioni tra pari della stessa età e con lo stesso background socioeconomico; colegas implica un livello di competizione tra persone giovani che non si confa all'idea di solidarietà che la definizione occidentale di 'amico' comporta. Il problema del significato della parola 'amico' dunque non comincia con Facebook, ma con la Torre di Babele.

Dopo aver completato un anno di ricerca sul campo, il gruppo ha trascorso il mese di maggio del 2014 a Londra, per consolidare i risultati e pianificare. Successivamente, i ricercatori sono tornati sul campo per trascorrervi gli ultimi tre mesi, sino alla fine di settembre (a parte Haynes che ha iniziato e finito un po' dopo). Abbiamo poi deciso di continuare comparazioni esplicite scrivendo libri individualmente, tutti con gli stessi capitoli a eccezione del Capitolo 5 dedicato al tema specifico di ciascuno. Abbiamo inoltre notato quanto spesso i lavori sui social media siano privi di illustrazioni; pertanto, ogni libro contiene un Capitolo 3 ampiamente dedicato a illustrare e ad analizzare ciò che le persone postano. 
Ci si aspetta che la maggior parte dei libri accademici siano in larga misura un dialogo con altri accademici. Il problema tuttavia risiede nel fatto che questo è di scarso interesse per chiunque non sia accademico o sia di diversa formazione disciplinare. Altri argomenti, come la storia, spesso rimandano questi dibattiti con altri accademici alle note a pie' di pagine o di chiusura, in modo da mantenere una narrazione chiara per il lettore. Abbiamo seguito il loro esempio. Nelle nostre nove monografie abbiamo fatto in modo che il testo principale fosse quanto più chiaro possibile riguardo alla descrizione e all'analisi dei 15 mesi di ricerca sul campo. A questo fine, la gran parte delle citazioni e delle discussioni di altri accademici sono nelle nostre note finali o compariranno in articoli su rivista. Molti di noi hanno anche deciso di scrivere una seconda monografia. Abbiamo sempre considerato il nostro tema come un telescopio. Possiamo usare la nostra etnografia per mettere a fuoco la natura dei social media. Possiamo anche usare i social media per ingrandire il focus verso l'esterno con una etnografia arricchita e ampliata. I libri pubblicati sino a ora rappresentano la prima strategia. I libri successivi saranno il risultato delle seconda.

\section{Questioni etiche}

Ci sono due forti impegni etici che riguardano la disseminazione di questa ricerca. Uno relativo al fatto che il nostro materiale doveva essere accessibile nelle lingue dei paesi dove abbiamo lavorato. L'altro, che alle persone di questi paesi, con basso reddito, non si doveva precludere la possibilità di leggerlo per ragioni di costi. Abbiamo scelto di lavorare con UCL Press, appena fondata, perché nata con l'impegno di offrire copie digitali open access con una Creative Common Licence, oltre a copie a stampa in vendita. Siamo stati anche molto felici di avere l'opportunità di creare un corso di lezioni libero on line (noto in gergo come MOOC) sulla piattaforma FutureLearn, sviluppata dalla UK Open University, e anche un sito web contenente il nostro materiale audiovisivo (www.ucl.ac.uk/why-we-post). In questi luoghi speriamo anche di sviluppare una presenza attiva di social media, per fornire ulteriori percorsi di discussione e per mostrare i nostri risultati. Qui si può commentare e contribuire al progetto. Attraverso la combinazione di queste diverse forme e di questi livelli di pubblicazione abbiamo anche provato a sviluppare una visione olistica di disseminazione della ricerca da accordare 
con la natura olistica della ricerca etnografica.

Altre questioni etiche emerse da questo progetto hanno condotto a un insieme di procedure formali e considerazioni informali. Come stabilito dall'originaria assegnazione da parte dell'ERC, la nostra proposta di ricerca doveva in primo luogo essere approvata dal comitato etico dell'University College London, e in seguito dai comitati etici di tutti i paesi nei quali avremmo lavorato, prima di essere firmata dall'ERC stesso. Questo include la necessità di un consenso scritto e orale da parte dei partecipanti. È la burocrazia dell'etica. In pratica, la nostra prima preoccupazione è stata quella di assicurarsi che si spiegasse con chiarezza a quanti vi avrebbero preso parte la natura del progetto e come sarebbero state usate le informazioni ottenute da loro. Infine, la nostra regola etica di base è semplice: niente di ciò che facciamo deve danneggiare le persone implicate.

A dire il vero, la maggior parte del materiale raccolto non è problematico e la maggior parte delle persone non ha ragione di preoccuparsi se altri sanno che loro hanno postano un meme o cosa hanno mangiato a pranzo. In effetti, i nostri informatori nella stragrande maggioranza dei casi preferiscono essere identificati. Sentiamo, tuttavia, che essi possono non essere consapevoli del potenziale abuso nel trattamento dei loro dati personali; pertanto abbiamo collegialmente deciso di rigettare questa preferenza, tranne che per i lavori video per i quali l'identificazione è credibile. Tutti i nostri libri, invece, si basano su materiale reso anonimo. Questo significa che siamo liberi di cambiare particolari individuali, tipo il lavoro svolto o il luogo di nascita, ma soltanto nella misura in cui queste alterazioni siano del tutto irrilevanti rispetto al particolare punto preso in considerazione. Nei casi in cui il sito della ricerca è una città di più ampie dimensioni, come Mardin o Alto Hospicio, usiamo il nome reale, mentre per luoghi più piccoli siamo ricorsi a uno pseudonimo.

Nell'era corrente di Google e della ricerca online è del tutto possibile che un lettore trovi il nome reale dei nostri siti, anche se noi non confermeremmo l'identificazione; l'unica ragione per l'anonimato riguarda chiaramente la protezione degli informatori, per cui se qualcuno facesse questo non potrebbe essere che per ragioni malevoli. In ogni caso, abbiamo introdotto livelli aggiuntivi di anonimato al livello personale. Quanto più riteniamo che l'informazione pubblicata potrebbe danneggiare un individuo, tanto più stringente è stato il processo di anonimizzazione, sia riguardo a noi sia riguardo ai nostri informatori. Gli inglesi, per esempio, ritengono rischioso 
pubblicare la foto di un bambino, pertanto tutti i bambini inglesi pubblicati in Visualising Facebook sono stati parzialmente mascherati per tener conto di questo tipo di paure. In altri siti le persone non avevano invece questo tipo di preoccupazioni.

La questione maggiore dal punto di vista etico riguarda la protezione dei partecipanti da eventuali conseguenze negative. Ne deriva che pochi fanno riferimento all'altro lato della medaglia. Quali sono gli effetti positivi della ricerca? Lo scopo primario di questo lavoro è l'educazione, per diffondere la conoscenza riguardo all'uso e alle conseguenze dei social media nel mondo. Come capita, anche al livello del lavoro sul campo, la nostra esperienza ci dice che quasi inevitabilmente la gente ha piacere di parlare e trascorrere del tempo con gli antropologi; la natura informale di questa relazione implica che le persone si sentano libere sia di fare domande agli antropologi sia di rispondere alle loro. Talvolta, proprio perché non siamo la loro famiglia (né insegnanti o burocrati statali), le persone riescono a parlare in modo catartico delle cose che stanno loro a cuore, in un modo più profondo di quanto non possano fare con persone che 'conoscono' davvero.

Crediamo con passione ai benefici positivi dell'antropologia in sé. In un mondo in cui le persone hanno scarse opportunità di conoscere e apprezzare la diversità del mondo in cui viviamo, tendiamo a presumere che gli altri siano come noi più di quanto non sia realmente e abbiamo solo una minima idea di quanto strani si possa apparire loro. Noi speriamo che leggendo questo libro, e tutti gli altri di questa serie, i lettori riusciranno a comprendere meglio i social media e che cosa sono diventati adesso attraverso il loro diverso uso nel mondo. Soprattutto, tuttavia, speriamo che la lettura di questi libri da parte di persone di tutto il mondo renda queste maggiormente consapevoli degli altri e più empatiche. Questo imperativo positivo è il nocciolo della nostra etica e della stessa antropologia. 


\section{Capitolo 4 I risultati della nostra indagine}

Il questionario che qui presentiamo conteneva in tutto 43 domande, l' $80 \%$ delle quali a risposta multipla e il resto con semplice risposta numerica, completabile con facilità in una decina di minuti. È stato testato diverse volte per essere certi che fosse aderente ai significati locali relativi a ciascuno dei siti. L'intenzione era quella di somministrare il questionario ad almeno 100 persone per sito, anche se in pratica questo è variato da 99 (per il nord del Cile) a 229 (per il Brasile), con un totale di 1199 risposte $^{1}$ per tutti e nove i siti. Abbiamo cercato anche di includere l'intera gamma dei livelli socioeconomici rilevanti per ciascun sito. Maggiori dettagli e ulteriori retroscena dell'indagine si trovano alla fine di questo capitolo. In tutti i casi le percentuali presentate in questo capitolo riguardano le persone che hanno risposto a una particolare domanda piuttosto che il totale indagato. Intendiamo i risultati come illustrativi del modo in cui le persone hanno risposto a una specifica questione e non devono essere interpretati come statisticamente significativi.

Abbiamo presentato solo quei risultati che sono comuni a tutti e nove i siti $^{2}$. Essi sono organizzati secondo cinque diversi temi. Come per tutto il libro, il paese e le etichette regionali sono usati per comodità di riconoscimento. Non sono mai intesi per fare riferimento a una popolazione omogenea. Per esempio, il nostro sito indicato come Turchia sud-orientale consisteva soprattutto sia di arabi che di curdi e relativamente pochi informatori turchi. In molti casi mettiamo in evidenza numeri che risultano particolarmente alti o bassi che poi cerchiamo di spiegare.

\section{Tema 1: relazioni sociali}

Come etnografi riteniamo che i risultati presentati in Fig. 4.1 in generale riflettono i punti di vista espressi dai nostri informatori durante la ricerca etnografica a proposito del loro modello generale di socialità. A Trinidad, in Brasile, in Italia e nel Cile settentrionale le persone tendono a considerarsi molto socievoli offline a confronto con quelle inglesi o della Cina rurale. 


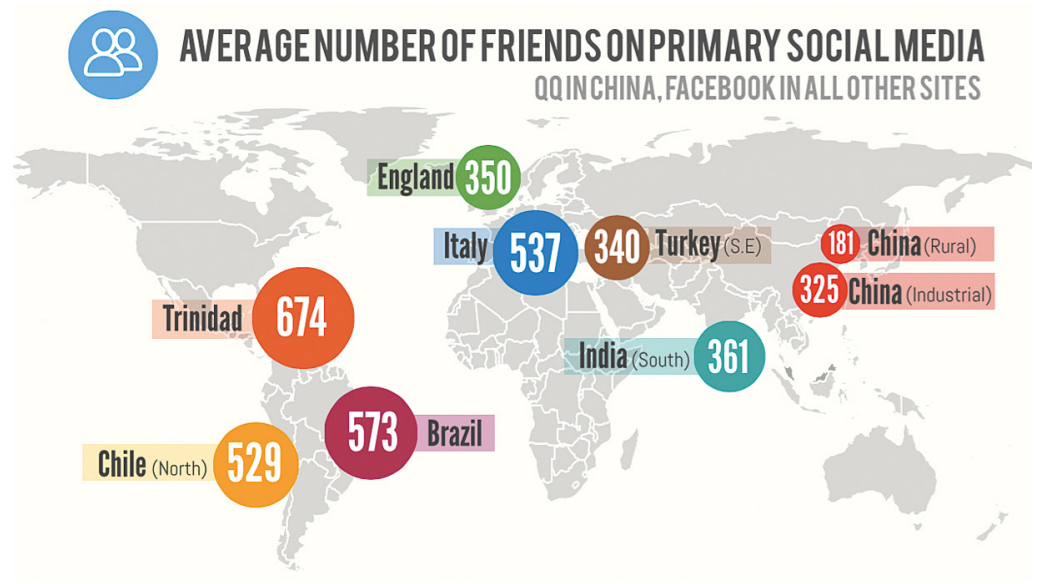

Fig. 4.1 Numero medio di amici su social media primari-QQ in Cina, Facebook in tutti gli altri siti

\section{DO YOU THINK...}

You know MORE people due to SOCIAL MEDIA ?

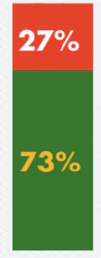

Brazil

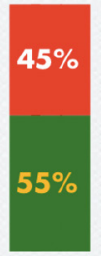

Chile

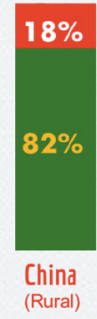

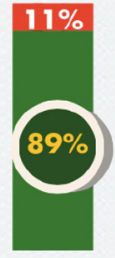
$\begin{array}{cl}\underset{\text { China }}{\text { (Industrial) }} & \text { India } \\ \text { (South) }\end{array}$

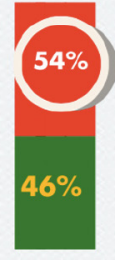

Italy Trinidad

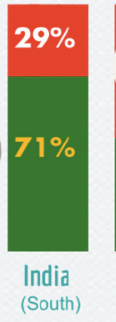

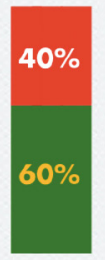

Turkey England

Fig. 4.2 Pensi di ... conoscere più persone grazie ai social media? Distribuzione delle risposte attraverso tutti i siti alla domanda se gli utenti ritenessero di conoscere più persone grazie ai social media

Mentre McDonald, in riferimento alla Cina rurale, scrive abbondantemente sull'importanza degli stranieri come categoria all'interno dei social media ${ }^{3}$, 
qui i contatti di questo tipo tuttavia non sono numericamente significativi. Viceversa, Wang rileva che per i lavoratori della Cina industriale che si spostano molto le loro connessioni online generalmente sono più stabili rispetto a quelle offline con i vicini e i colleghi temporanei che incontrano nel posto di lavoro. Considerate le restrizioni che questi lavoratori affrontano nelle loro vite offline, i social media diventano il centro della loro socializzazione. Spesso valutano questi contatti online più di quelli offline. Inoltre, dal momento che la maggior parte di questi lavoratori migranti hanno meno di 30 anni, i social media per loro diventano il luogo in cui esprimere meglio se stessi e esplorare stili di vita 'moderni'. Questi risultati sono confermati pure da una inchiesta indipendente condotta da Wang nel suo sito ${ }^{4}$ in Inghilterra, al contrario, le persone tendono ad essere caute e diffidenti nelle interazioni con persone che non conoscono già offline, in parte a causa delle tante storie che circolano nei media inglesi sulle conseguenza problematiche di fare amicizia con stranieri. Leccezione a questo è costituita dai dati di siti come Tinder.

Gli italiani sono altamente socievoli offline e considerano i social media soprattutto come una estensione dei loro rapporti con persone che già conoscono nelle loro città di residenza. Il 54\% delle risposte fornite suggerisce che più del 60\% degli amici su Facebook appartiene alla stessa città. A parte una frazione altamente coltivata che è in contatto con italiani di altre regioni, questi utenti non si aspettano che l'uso dei social possa portare loro nuove connessioni o che un numero consistente di contatti offline si traduca necessariamente in contatti online (fig. 4.3).

\section{DO YOU THINK...}

Individuals with more OFFLINE friends also have more friends ONLINE?
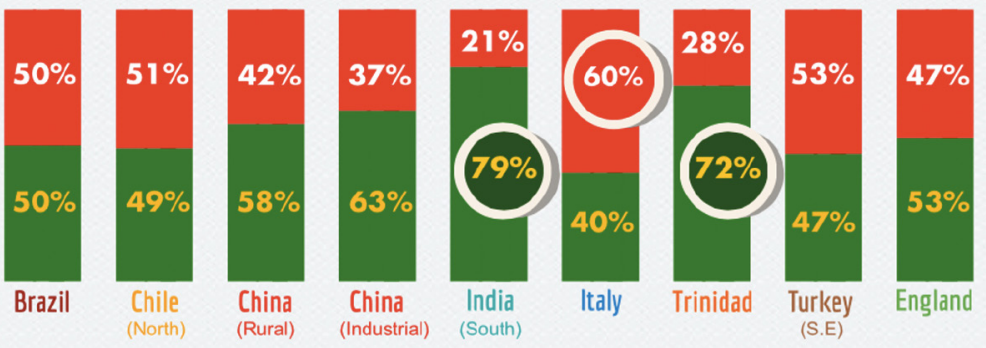

India

Italy

Trinidad

Turkey

England

Fig. 4.3 Pensi che ... gli individui con più amici offline abbiano anche più amici online? Distribuzione delle risposte alla domanda se gli utenti ritenessero che avere molti amici offline avesse come risultato averne molti on line 
D'altro canto, coloro che hanno risposto al questionario a Trinidad considerano equivalente avere più amici offline e avere reti estese fuori dalla propria città, per esempio nei casi in cui si frequenti l'università o si lavori fuori oppure se si hanno famiglia e amici oltremare. In generale i siti di ricerca in America latina o a Trinidad erano quelli in cui le persone, usando i social media, si aspettavano di estendere le loro reti sociali, soprattutto attraverso la procedura dello stringere amicizia con amici degli amici. Questa convinzione si rifletteva pure nel più gran numero di amicizie on line come nella Fig. 4.1. Qui c'è una gradazione. Nell'India del sud gli amici di amici con cui non ci si incontra offline sono assimilati alla categorie di estranei, ma non c'è un chiaro desiderio di entrare in contatto con estranei come è stato rilevato per il sito della Cina rurale.

Le differenze nelle risposte a questa domanda (Fig. 4.4) ci forniscono uno dei più decisivi esempi del perché pensiamo che queste domande ci insegnano qualcosa di diverso da quanto ci si aspetti. In Brasile, Spyer si aspettava una risposta molto diversa, dal momento che la sua etnografia aveva mostrato chiaramente che le persone del suo sito prima di diventare amici di altri non chiedevano il permesso alla famiglia o agli amici. Tuttavia, la risposta ha reso chiaro il fatto che anche se non chiedono il permesso per aggiungere amici, spesso si rivolgono alla famiglia e agli amici per scoprire chi sia realmente la persona che manda loro una richiesta di amicizia 5 . Mentre è vero sia per il Cile settentrionale sia per Trinidad il fatto che le persone accettavano che un amico di un amico fosse un potenziale amico, non ritenevano che questo equivalesse a consultarsi con altre persone così come la domanda chiedeva. Ciò indica che per quanto si prendessero misure per provare a formulare le domande nello stesso modo per tutti i casi, in pratica le persone interpretavano le implicazioni in modo diverso. La variazione delle loro risposte riflette dunque questa differenza di interpretazione e non necessariamente una differenza nella pratica. Ancora una volta, è possibile rendersi conto di questo conoscendo già molto bene le pratiche degli informatori attraverso l'etnografia più ampia. 


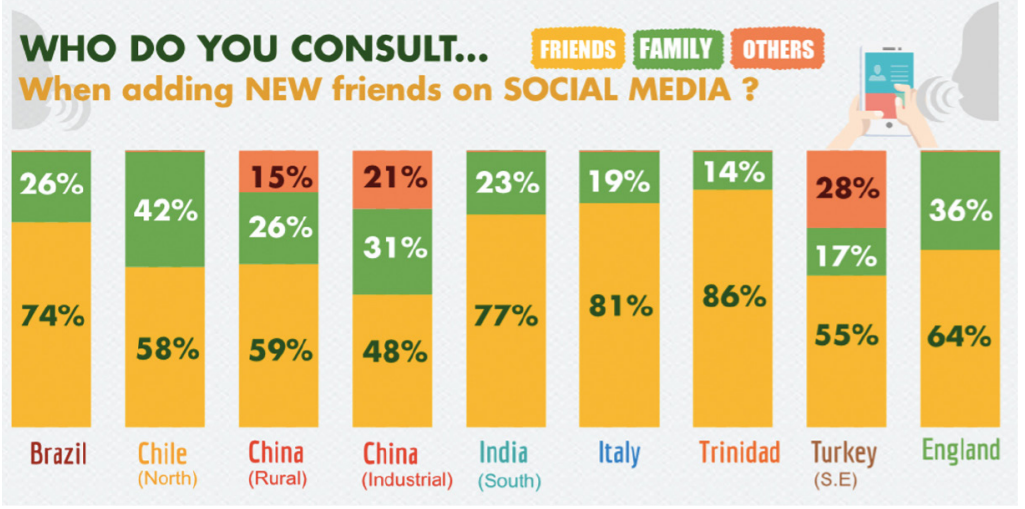

Fig. 4.4 Chi consulti ... se aggiungere nuovi amici sui social? Amici Famiglia Altri. Distribuzione delle risposte alla domanda su chi gli utenti consultassero nell'aggiungere nuovi amici sui social media

La sezione successiva dell'indagine comprende tre domande per aiutare a stabilire ulteriormente il livello di coinvolgimento della famiglia e degli amici nell'uso dei social media da parte dei nostri informatori.

La tabella 4.1 riflette le competenze tecniche di chi risponde. Nel nostro sito inglese, tutti coloro che facevano settare il loro account da altri erano persone anziane, come dimostra il fatto che uno dei gruppi principali (il 6\%) per l'assistenza era costituito dai loro figli. Le persone più giovani potevano provare imbarazzo nel dire di non essere capaci di farlo da sole. Viceversa, le persone della Cina industriale, con un livello di istruzione molto limitato, potevano aspettarsi di trovare un aiuto in un internet café, ormai un punto importante per imparare qualcosa su internet. Wang nota come i negozi di cellulari stiano gradualmente assumendo questo ruolo dal momento che l'accesso a internet tramite smartphone sta sensibilmente crescendo. Entrambi questi aspetti sono in netto contrasto con quanto accade nel nostro sito brasiliano. Qui, la natura sociale dei social media è rafforzata dal fatto che settare un account è in sé un progetto sociale, finalizzato ad approfondire e a estendere relazioni di amicizia esistenti. 


\section{WHO set up your FIRST} SOCIAL MEDIA ACCOUNT ?

myself ' friends ' parents 'siblings' partner'children' other 'Internet

\begin{tabular}{|c|c|c|c|c|c|c|c|c|}
\hline C) & myself & friends & parents & siblings & partner & children & $\begin{array}{l}\text { other } \\
\text { relatives }\end{array}$ & $\begin{array}{l}\text { Internet } \\
\text { Cafe worker }\end{array}$ \\
\hline Brazil & $41 \%$ & $39 \%$ & $1 \%$ & $7 \%$ & $4 \%$ & $3 \%$ & $0 \%$ & $6 \%$ \\
\hline Chile (North) & $74 \%$ & $6 \%$ & $1 \%$ & $9 \%$ & $2 \%$ & $6 \%$ & $0 \%$ & $1 \%$ \\
\hline China(Rural) & $48 \%$ & $18 \%$ & $1 \%$ & $19 \%$ & $5 \%$ & $3 \%$ & $5 \%$ & $1 \%$ \\
\hline China (Industrial) & $20 \%$ & $41 \%$ & $0 \%$ & $15 \%$ & $1 \%$ & $2 \%$ & $9 \%$ & $12 \%$ \\
\hline India (South) & $48 \%$ & $23 \%$ & $3 \%$ & $17 \%$ & $5 \%$ & $4 \%$ & $0 \%$ & $1 \%$ \\
\hline Italy & $65 \%$ & $12 \%$ & $2 \%$ & $9 \%$ & $6 \%$ & $6 \%$ & $0 \%$ & $0 \%$ \\
\hline Trinidad & $62 \%$ & $25 \%$ & $0 \%$ & $7 \%$ & $3 \%$ & $2 \%$ & $0 \%$ & $1 \%$ \\
\hline Turkey (S.E) & $74 \%$ & $17 \%$ & $0 \%$ & $6 \%$ & $0 \%$ & $2 \%$ & $0 \%$ & $1 \%$ \\
\hline England & $83 \%$ & $7 \%$ & $1 \%$ & $1 \%$ & $2 \%$ & $6 \%$ & $0 \%$ & $0 \%$ \\
\hline
\end{tabular}

Tabella 4.1 Chi ti ha messo a punto il tuo primo account sui social media? Io stesso - Amici - Genitori - Fratelli - Partner - Figli Altri parenti - Un addetto di un internet point

WHO posts regularly on your SOCIIL MEDII?

\begin{tabular}{|r|c|c|c|c|c|c|}
\hline Brazil & $5 \%$ & $32 \%$ & $14 \%$ & $2 \%$ & $47 \%$ & $0 \%$ \\
\hline Chile (North) & $9 \%$ & $13 \%$ & $23 \%$ & $7 \%$ & $49 \%$ & $0 \%$ \\
\hline China (Rural) & $10 \%$ & $40 \%$ & $7 \%$ & $2 \%$ & $41 \%$ & $0 \%$ \\
\hline China (Industrial) & $4 \%$ & $25 \%$ & $1 \%$ & $11 \%$ & $59 \%$ & $0 \%$ \\
\hline India (South) & $3 \%$ & $15 \%$ & $9 \%$ & $5 \%$ & $68 \%$ & $0 \%$ \\
\hline Italy & $4 \%$ & $18 \%$ & $10 \%$ & $6 \%$ & $62 \%$ & $0 \%$ \\
\hline Trinidad & $6 \%$ & $15 \%$ & $15 \%$ & $4 \%$ & $60 \%$ & $0 \%$ \\
\hline Turkey (S.E) & $1 \%$ & $8 \%$ & $13 \%$ & $1 \%$ & $49 \%$ & $28 \%$ \\
\hline England & $19 \%$ & $10 \%$ & $14 \%$ & $12 \%$ & $45 \%$ & $0 \%$ \\
\hline
\end{tabular}

Tabella 4.2. Chi posta regolarmente sui tuoi social media? Genitori - FratelliPartner - Figli-Amici-Nessuno. Chi tra i familiari e gli amici posta regolarmente sui tuoi social? 
Nel caso cinese, il numero contenuto nella tabella rappresenta la percentuale di persone che interagiscono più regolarmente sui social, mentre postare direttamente sul sito di un'altra persona non è un profilo della piattaforma cinese. Pur nondimeno, è difficile immaginare un risultato più imprevedibile di quello relativo al più alto numero di post da parte di fratelli provenienti da un sito rurale cinese soggetto alle restrizioni del Governo in merito alla pianificazione familiare di un figlio per famiglia. McDonald suggerisce che si tratta di una combinazione di due fattori. In primo luogo, coloro che hanno fratelli forse condividono con loro relazioni molto strette, in secondo luogo il numero fa riferimento a una pratica cinese per cui è comune riferirsi ai cugini appellandoli fratelli. Questo è pertanto un ulteriore avvertimento in merito alla presunzione che sia possibile prevenire interpretazioni locali delle domande che si sforzano di essere comuni a contesti di culture diverse.

In Italia i social media sono considerati piattaforme molto pubbliche e sono viste principalmente come piattaforme di connessione tra amici piuttosto che con la famiglia. Il termine italiano 'amici' è ampio e include $\mathrm{i}$ migliori amici, gli amici della città in cui si vive, i compagni di scuola, i colleghi di lavoro. Similmente, a Trinidad i numeri sembrano riflettere ciò che si era capito all'inizio: le persone hanno un numero relativamente alto di amici su Facebook con i quali socializzano sia online che offline. Nel sud dell'India postare giornalmente sui profili degli amici è considerato come un'estensione della socialità, mentre la maggior parte delle comunicazioni con i membri più stretti della famiglia si fa con altri media come il telefono e i servizi di messaggistica. Qui l'anomalia è il numero (28\%) che si riferisce ai siti della Turchia meridionale dove nessuno posta sui social. Esito plausibile dal momento che riflette il problema locale del mostrarsi in pubblico, tradizionalmente sotto stretto controllo e la cui infrazione è considerata come un affronto alla modestia. Costa ha scoperto che molte persone generalmente preferivano postare sulla propria bacheca perché in ansia per la possibile reazione del destinatario a quanto era stato postato da altri sul proprio profilo. 


\begin{tabular}{|c|c|c|c|c|c|c|}
\hline \multicolumn{7}{|c|}{ With WHOM do you have arguments over } \\
\hline & & \multicolumn{2}{|c|}{ their use of } & \multicolumn{3}{|c|}{ SOCIRL MEDIE } \\
\hline$r$ & parents & siblings & partner & children & friends & $\begin{array}{l}\text { other } \\
\text { relatives }\end{array}$ \\
\hline Brazil & $24 \%$ & $17 \%$ & $38 \%$ & $3 \%$ & $18 \%$ & $\mathrm{O} \%$ \\
\hline Chile (North) & $30 \%$ & $8 \%$ & $28 \%$ & $16 \%$ & $18 \%$ & $0 \%$ \\
\hline China (Rural) & $24 \%$ & $21 \%$ & $8 \%$ & $10 \%$ & $29 \%$ & $7 \%$ \\
\hline China (Industrial) & $26 \%$ & $2 \%$ & $48 \%$ & $7 \%$ & $11 \%$ & $7 \%$ \\
\hline India (South) & $25 \%$ & $16 \%$ & $12 \%$ & $6 \%$ & $41 \%$ & $0 \%$ \\
\hline Italy & $26 \%$ & $15 \%$ & $11 \%$ & $7 \%$ & $41 \%$ & $0 \%$ \\
\hline Trinidad & $18 \%$ & $15 \%$ & $24 \%$ & $4 \%$ & $38 \%$ & $\mathrm{O} \%$ \\
\hline Turkey(S.E) & $14 \%$ & $13 \%$ & $32 \%$ & O\% & $41 \%$ & $0 \%$ \\
\hline England & $22 \%$ & $16 \%$ & $18 \%$ & $4 \%$ & $40 \%$ & $0 \%$ \\
\hline
\end{tabular}

Tabella 4.3 Con chi hai discussioni sul modo di usare i social media? GenitoriFratelli-Partner-Figli-Amici-Altri parenti. Persone con cui si hanno discussioni sul proprio uso dei social media

In Brasile generalmente si ritiene che i social media abbiano condotto a maggiore infedeltà e sospetto. Qui, come a Trinidad, i social sono visti come un mezzo usato dai partner per spiarsi reciprocamente. Nel sito inglese c'era una certa evidenza etnografica relativa all'uso dei social per discutere tra compagni di scuola. Dato che nella maggioranza dei nostri siti gli amici erano al centro dell'uso dei social media, volevamo capire se anche coloro che rispondevano ai nostri questionari avessero tolto l'amicizia dai loro profili a qualcuno e se questo fosse correlabile all'argomento topico dei social, la politica. 
Have you ever UN-FRIENDED someone on social media because of a POLITICAL POSTING they made?

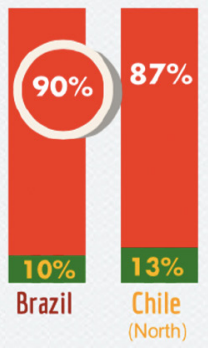

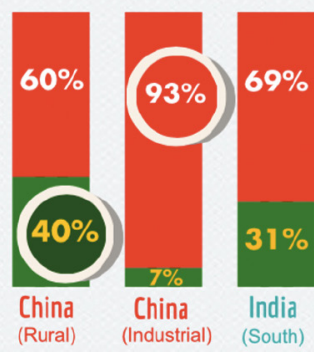

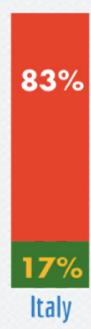

Italy

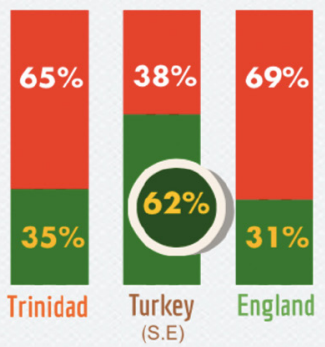

Fig. 4.5 Hai mai tolto l'amicizia a qualcuno sui social media per aver postato post politici? Distribuzione delle risposte alla domanda se l'utente avesse mai tolto l'amicizia a qualcuno per post politici

Le risposte a questa domanda forniscono un buon esempio del perché i nostri due siti cinesi non dovrebbero essere tenuti insieme come 'cinesi': riguardo al togliere l'amicizia per discussioni politiche, il sito della Cina rurale presenta uno dei numeri più alti e quello della Cina industriale il più basso. Wang fu sorpreso dal fatto che un uguale $7 \%$ aveva tolto l'amicizia a qualcuno su questi terreni e suggeriva che probabilmente si trattava dei proprietari delle industrie e dei manager piuttosto che degli operai.

Ciò è dovuto in parte al fatto che, nel primo posto, le persone si astengono dal postare su argomenti politici. L'eccezione sarebbe rappresentata dal postare su corruzione, regolamentazione locale, inquinamento e status sociale iniquo tra i migranti rurali, ingiustizia e povertà, dal momento che tutte queste questioni sono un modo per stringere legami con altri migranti. Viceversa, gli informatori della Cina rurale toglieranno l'amicizia a quelli che postano su qualsivoglia questione politica, fatto considerato inopportuno. Lì le persone pensano che i social media siano un luogo per condividere felicità, per esempio fotografie di bambini, memi romantici o foto di luoghi turistici, piuttosto che per lamentare cose di cui si è insoddisfatti. Il loro problema non è la paura delle ripercussioni da parte dello stato, ma della disapprovazione da parte dei loro amici. Anche nel nostro sito brasiliano la politica è considerata come qualcosa che è meglio lasciare ai politici e che 
non vale davvero la pena discutere sui social, ma essa raramente costituisce motivo per togliere l'amicizia.

Il nostro sito nella Turchia sudorientale tuttavia è del tutto diverso. Qui la politica non solo conta, ma è anche molto importante e argomento sensibile. In questo sito le divisioni politiche sono nettamente marcate. Considerate la storia e la presenza del conflitto curdo, qui le persone si schierano o con i curdi o con il governo. Molti degli informatori di Costa hanno parenti che sono stati arrestati, torturati o uccisi dalla polizia o dall'esercito tra gli anni Ottanta e Novanta del Novecento. Persone che sostengono gruppi politici diversi spesso sono amiche su Facebook nello stesso modo in cui condividono gli stessi edifici, lo stesso vicinato, le scuole o l'ufficio in cui lavorano. Considerata la forte dimensione politica sotterranea, esse evitano consapevolmente di discutere, sia online che offline, argomenti che potrebbero creare disaccordo e conflitti. Quando però condividono post politici sui social, è molto probabile che si crei conflitto; togliere l'amicizia da Facebook in questi casi è il modo più semplice per gestirlo.

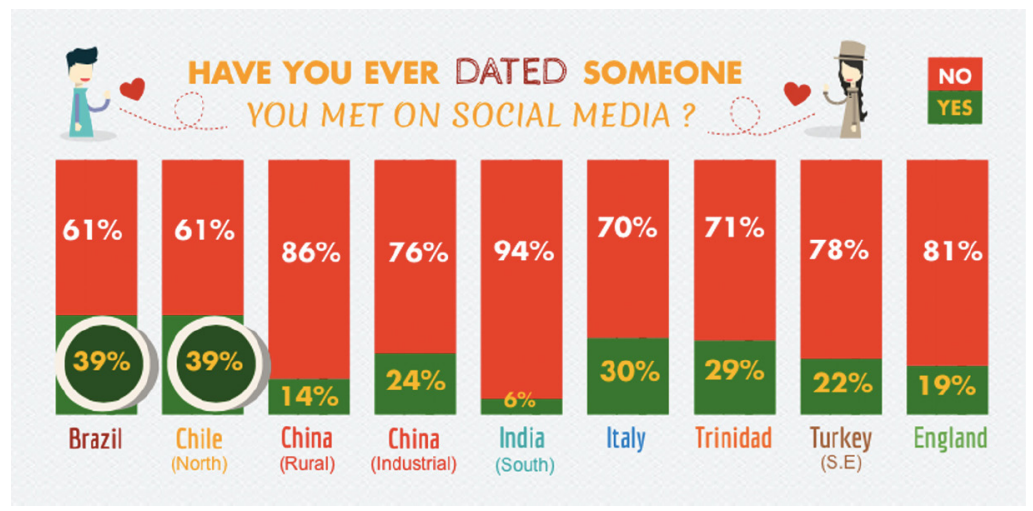

Fig. 4.6 Sei mai uscito con qualcuno che hai incontrato sui social media? Distribuzione di appuntamenti presi

Infine, nell'ambito del tema delle relazioni sociali, volevamo capire la possibilità di incontri attraverso i social media in riferimento a tutti e nove $\mathrm{i}$ siti. Come abbiamo già rilevato all'inizio di questo capitolo, in America Latina e a Trinidad i social media sono strettamente connessi alla possibilità di stringere o rompere relazioni. La prima storia del libro di Miller, Tales 
from Facebook ${ }^{6}$, si incentra su una questione di gelosia in una relazione a Trinidad. Questo è meno comune altrove. In Inghilterra Tinder è diventato importante soprattutto dopo la fine della nostra ricerca sul campo, mentre Grindr era già ben avviato per omosessuali maschi (anche se non tutti considerano Grindr o Tinder social media). Momo è usato in Cina soprattutto nelle aree urbane piuttosto che nei siti della nostra ricerca. Una delle ragioni per cui nel nostro sito del nord del Cile le persone cercavano di trovare partner in questo modo era, come affermavano scherzando, la loro pigrizia. Era più facile chattare con qualcuno on line piuttosto che avvicinarlo di persona, perché si avevano minori possibilità di essere rifiutati. Inoltre, il sito nord cileno offriva pochi spazi sociali quali bar o club in cui incontrare potenziali partner, anche se le persone si sentivano più a loro agio nell'incontrare amici di amici piuttosto che perfetti sconosciuti. Come ha spiegato Miguel, un informatore del nord del Cile:

Le persone non si fidano dei siti per incontri, tipo Tinder. Non sai chi sia la persona. Non puoi vedere i suoi amici...Se stai guardando qualcuno su Facebook, puoi vedere anche i suoi amici condivisi, di dove sono, quali sono i loro passatempi. Così, ti fai un'idea della persona, di chi sono realmente. Sai che non sono finti.

Nel sud dell'India, agli uomini sarebbe piaciuto avere appuntamenti attraverso i social, e questo potrebbe accadere tra la classe media o tra gli addetti all'Information Technology (IT). Tuttavia, anche se un certo numero flirta sui social, è molto raro che questo si traduca in un qualsivoglia tipo di relazione offline, a parte il matrimonio, a causa delle molte restrizioni riguardanti il comportamento delle donne.

\section{Tema 2: attività sui social media}

Una delle principali attività sui social consiste nel postare materiali visivi tipo foto e memi. La nostra prima domanda su questo tema riguardava espressamente questo aspetto. 


\section{WHAT PERCENTAGE of PHOTOS}

that you take end up getting posted on your social media profiles?

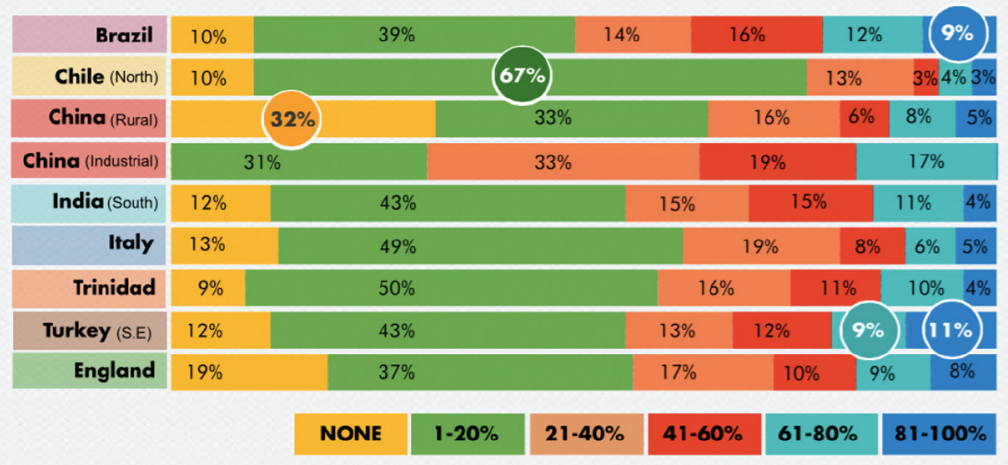

Fig. 4.7 Che percentuale di foto che fai finisce postata sui tuoi profili sui social media? Distribuzione della percentuale di foto postate sui social media

La Fig. 4.7 suggerisce netti contrasti tra i nove siti. Mentre il 67\% degli intervistati nel Nord del Cile posta meno del $20 \%$ di proprie fotografie, in Turchia sud orientale il $20 \%$ ne posta il $60 \%$. D'altro canto, il $32 \%$ delle risposte dal sito della Cina rurale indica che nessuna delle foto scattate finisce sui social.

Le ragioni riguardano le differenze nel modo di intendere quale sia il materiale ritenuto adeguato per essere postato. Il criterio chiave, in molti posti incluso il Cile, è lo humor. Le persone condividono cose che sono divertenti e per questo motivo attraggono commenti. I social media sono considerati una sorta di intrattenimento. Per esempio, le persone postano memi nella sezione dei commenti per un meme di guerra, generando centinaia di risposte, mentre una fotografia di cibo, o di attività pomeridiane o un selfie produrrà al massimo 15 commenti. Pertanto, mentre gli utenti qui continuano a fare scatti per il loro piacere personale e per i posteri, sui social media l'enfasi riguarda il visuale che genera un grande numero di interazioni.

Questo è persino maggiormente vero nel nostro sito della Cina rurale dove le persone non condividono le proprie fotografie sui social, con l'eccezione delle teenager e delle donne giovani che condividono selfie. Generalmente si preferisce condividere memi e post divertenti. McDonald ritiene che qui, 
come nel Cile del Nord, si pensa che questo tipo di post siano di maggior interesse generale. Un'altra ragione è la privacy. Per molti, le poche fotografie personali e della propria famiglia condivise online spesso sono protette da password, accessibili solo da coloro che si conoscono personalmente.

Riguardo alle attività, la domanda successiva riguardava i giochi on line.

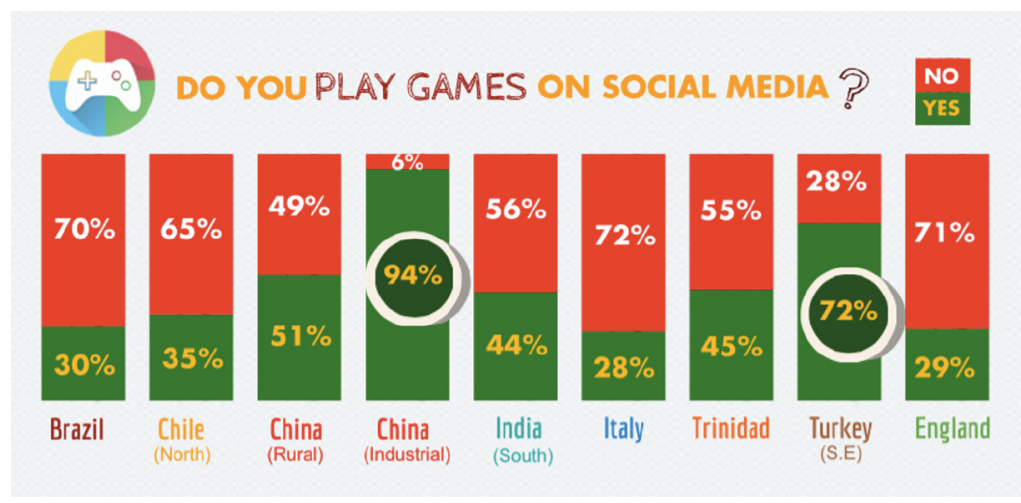

Fig. 4.8 Giochi sui social media? Distribuzione di gioco on line sui social

Qui risalta il numero riguardante la Cina industriale. Questo è probabilmente il sito in cui la giornata lavorativa delle persone comporta un lavoro incessante. Non deve pertanto stupire se esse ricorrono al gioco per rilassarsi e staccare dal lavoro. Ciò riflette infatti una più ampia enfasi sull'uso degli smartphone per l'intrattenimento in generale, un tratto che emerge dall'ulteriore inchiesta di Wang sull'uso degli smartphone tra i 200 intervistati al telefono ${ }^{7}$. Questi operai in genere non hanno altro tempo, né denaro né energie da dedicare alla vita sociale dopo lunghe ore di pesante lavoro. Nello stesso tempo, oltre alla possibilità di rilassarsi che questo tipo di giochi comporta, giocare è considerato anche il modo principale di tenere relazioni online con gli amici, specialmente tra i giovani maschi. 


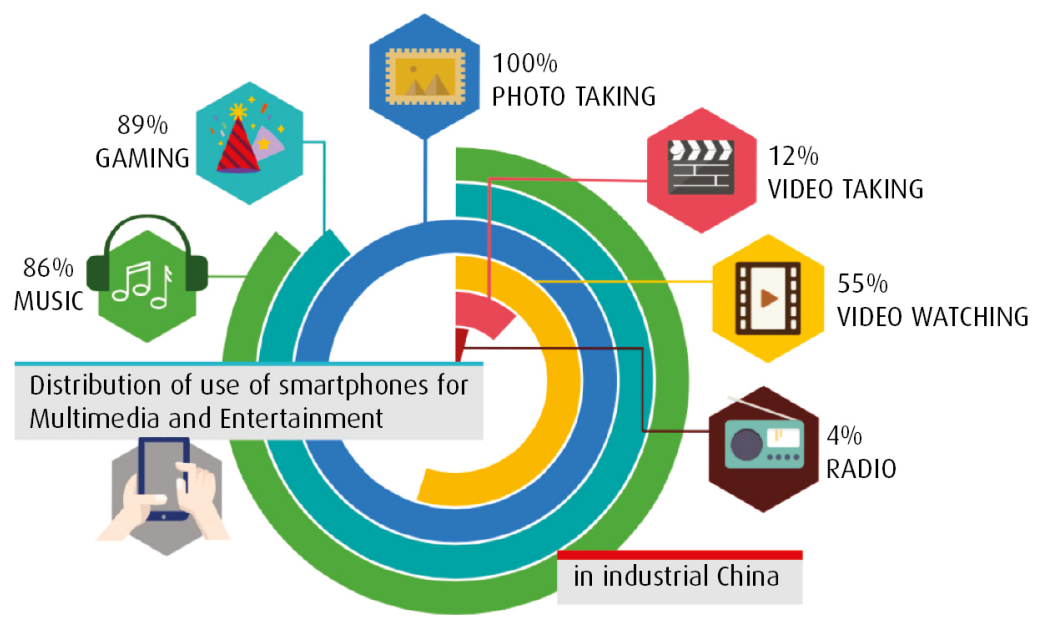

Fig. 4.9 Distribuzione dell'uso di smartphone per multimedia e intrattenimento nella Cina industriale. Musica - Gioco - Foto - Videoriprese - Video - Radio

Il gioco on line è un aspetto molto importante dei social (soprattutto Facebook) anche nella Turchia sudorientale. I giochi più comuni sono Candy Crush Saga, Ok e Taula. Giocare è un modo per socializzare con vecchi e nuovi amici. Le persone giocano on line non solo con gli amici ma anche con estranei, con la possibilità che questi estranei, giocando, possano diventare nuovi amici. Il gioco online è usato anche per flirtare in modo discreto con persone del sesso opposto. Per i giovanissimi (per esempio, bambini della scuola elementare, tra gli 8 e gli 11 anni) giocare è probabilmente una delle ragioni principali per usare i social. Molti agricoltori in Turchia giocano a Farmville, il primo gioco davvero di successo in Facebook. In Italia, invece, i giochi di casa (Xbox, PlayStation, Wii) sono ancora importanti e hanno la funzione di stringere legami all'interno del gruppo familiare.

\section{Tema 3: la privacy}

Un terzo ambito, che spesso le persone ritengono implicato con i social media e che per questo abbiamo indagato, riguarda la privacy. 


\section{Percentage of people who shared their SOCIAL MEDIA PASSWORDS with family/friends}

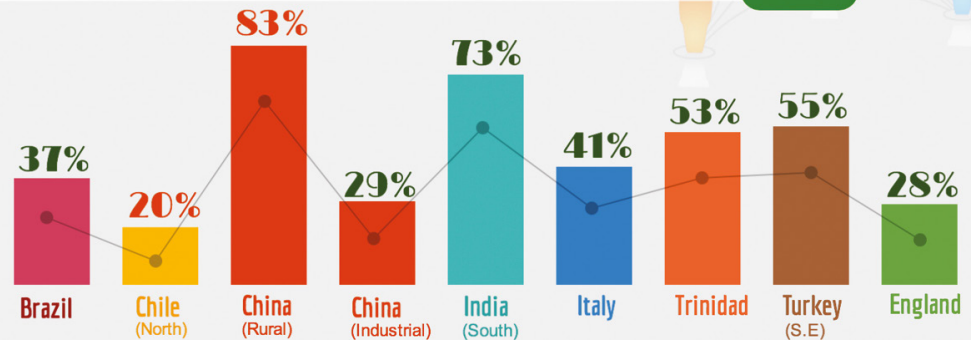

Fig. 4.10 Percentuale di persone che condividono la loro password di accesso ai social con famiglia / amici

Dalla Fig. 4.10 risulta evidente che gli intervistati dei diversi siti hanno visioni diverse riguardo alla possibilità di condividere con altri la loro password di accesso ai social. Come si può vedere, il dato varia da uno su cinque che lo fa, nel Cile del nord, a quattro su cinque nel nostro sito della Cina rurale. Tuttavia, ne capiamo di più considerando in dettaglio quali sono le persone con cui sono pronti a condividere la loro password.

\begin{tabular}{|c|c|c|c|c|c|}
\hline C) & partner & parents & siblings & children & friends \\
\hline Brazil & $48 \%$ & $7 \%$ & $19 \%$ & $6 \%$ & $20 \%$ \\
\hline Chile (North) & $50 \%$ & $10 \%$ & $10 \%$ & $5 \%$ & $25 \%$ \\
\hline China (Rural) & $30 \%$ & $10 \%$ & $22 \%$ & $10 \%$ & $27 \%$ \\
\hline China (Industrial) & $12 \%$ & $38 \%$ & $50 \%$ & $0 \%$ & $0 \%$ \\
\hline India (South) & $30 \%$ & $3 \%$ & $11 \%$ & $8 \%$ & $48 \%$ \\
\hline Italy & $42 \%$ & $4 \%$ & $18 \%$ & $24 \%$ & $11 \%$ \\
\hline Trinidad & $46 \%$ & $6 \%$ & $11 \%$ & $7 \%$ & $30 \%$ \\
\hline Turkey(S.E) & $46 \%$ & $7 \%$ & $17 \%$ & $6 \%$ & $24 \%$ \\
\hline England & $75 \%$ & $4 \%$ & $0 \%$ & $7 \%$ & $14 \%$ \\
\hline
\end{tabular}

Tabella 4.4 Con chi condividi la tua password di accesso ai social media? Partner-Genitori - Fratelli - Figli-Amici. Persone con cui gli utenti condividono la loro password di accesso ai media 
Molti siti registrano un'alta percentuale nel caso di condivisione della password con il partner, cosa che si può spiegare evocando la percezione (in alcuni dei nostri siti) che l'uso dei social possa facilmente condurre all'infedeltà favorendo l'amicizia con gli estranei. Condividere la password con il partner consente così di monitorare l'account del partner. In alcuni siti come nel sud dell'India, le coppie hanno un solo profilo sui social e dunque la condivisione della password è inevitabile.

Nel nostro sito del nord del Cile, l'intera famiglia estesa, dai nipoti ai nonni, può condividere l'uso dello stesso computer. Il cellulare pertanto può essere il solo segmento di privacy. Anche se molte coppie sperimentano la gelosia riguardo all'uso che il proprio partner fa dei social, la condivisione della password raramente è considerata obbligatoria. Le persone rispettano la privacy del partner, riferendo spesso che preferiscono non sapere se il loro partner abbia occasionalmente contatti con ex o flirti on line. Un meme sintetizza questo sentimento, come illustrato dalla Fig. 4.11.

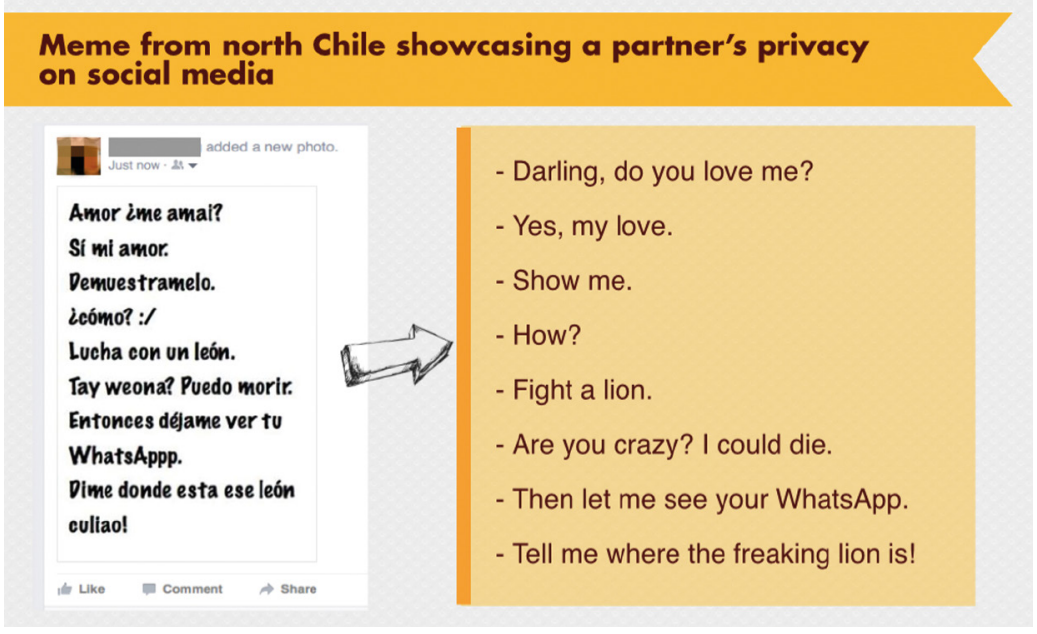

Fig. 4.11 Meme dal Cile del Nord che esibisce la privacy di un partner sui social media

Nella Cina rurale molti condividono la password con i loro amici mentre sono a scuola, non soltanto per stabilire rapporti di fiducia ma anche per far sì che gli amici possano aiutarli occupandosi del loro profilo QZone (entrandovi e sostenendo quei giochi per cui è necessario assicurarsi una crescita costante di livelli). 
La Fig. 4.12 mostra che, a eccezione del sito inglese, c'erano gruppi di persone che usavano profili falsi o resi anonimi. La situazione cinese è molto diversa dal momento che i social media generalmente non si basano sull'uso del nome proprio. Il nome proprio generalmente è usato per gli account di lavoro, visto che molte industrie usano i social per comunicare con gli operai. Per l'uso 'privato' dei social, molti usano un $Q Q$ name anonimo, che consente un ampio margine di libertà di parola. Le persone hanno pure account multipli per scopi diversi, per giocare o per poter frequentare molti siti diversi, come si evince dalla Fig. 4.13.

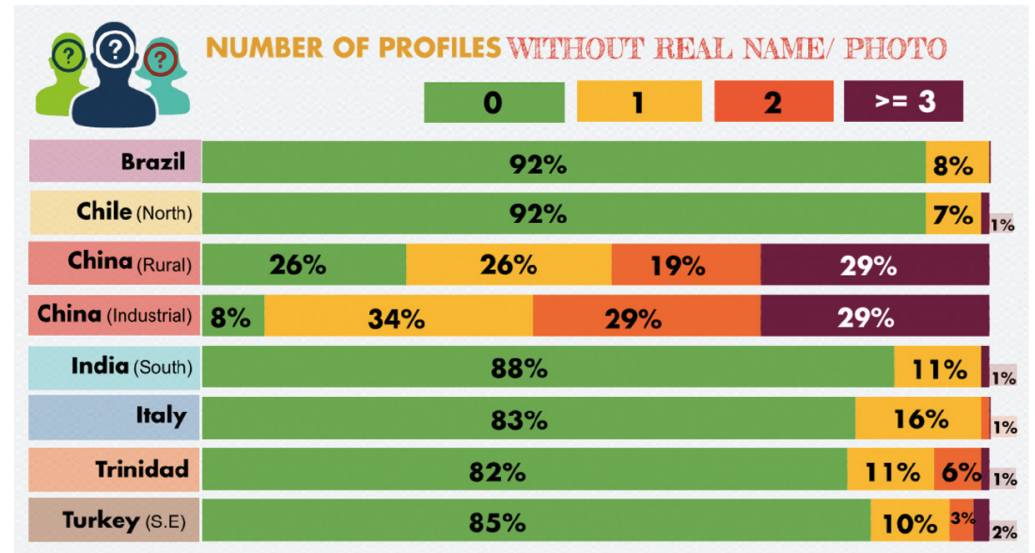

Fig. 4.12 Distribuzione di profili social senza nome proprio / foto

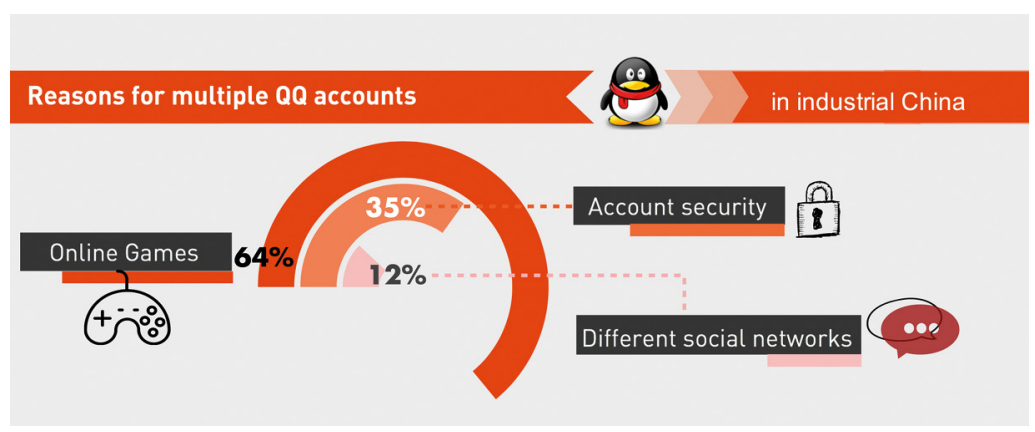

Fig. 4.13 Ragioni per account QQ multipli nella Cina industriale.

Giochi on line-Sicurezza - Social network diversi 
I partecipanti del sito di McDonald generalmente ritenevano che se qualcuno è un vero amico dovrebbe essere in grado di riconoscerti dal tuo avatar/nome utente. Come tale, questa convenzione funzionava anche come un modo per testare l'amicizia. Le piattaforme cinesi di social come QQ e WeChat consentono anche di 'etichettare' l'account di un amico con un nickname dei propri nei casi in cui non si riconosca di chi sia l'account. L'uso di avatar facilita le relazioni di amicizia con estranei, fornendo una 'maschera' dietro cui possono svolgersi le interazioni inziali.

Il problema principale con questa domanda riguarda il fatto che le persone che usano account multipli molto probabilmente non vogliono rivelarlo per una indagine. Costa sicuramente ha avvertito la non accuratezza dei suoi risultati per il suo sito della Turchia sudorientale. Ammettiamo che falsi profili potrebbero danneggiare la propria reputazione. Riconoscere ciò da parte di un uomo comportava una parziale ammissione di andare attivamente a caccia di donne e di molestarle, mentre ammetterlo da parte delle donne significava dichiarare apertamente che si aveva qualcosa da nascondere.

Una diversa fonte di ansietà era rappresentata dalla possibilità che altri potessero caricare foto sui social senza permesso o a insaputa degli interessati. Questo punto è indagato nella Fig. 4.14.

\section{Are you WORRIED about people}

pulting photos of yourself on SOCIAL MEDIA?

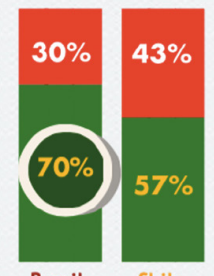

Brazil

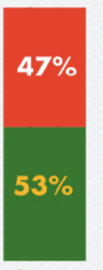

China

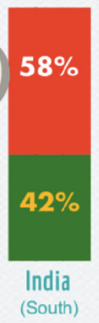

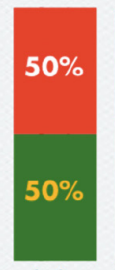

Italy

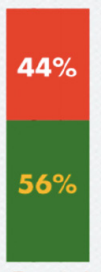

Trinidad

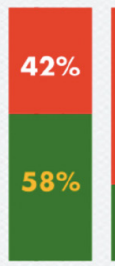

Turkey England

Fig. 4.14 Ti preoccupa che le persone possano mettere foto tue sui social media?

Distribuzione delle risposte alla domanda se gli utenti fossero preoccupati che le persone mettessero sui social loro foto

Nella Cina industriale le persone in genere sono felici di vedere loro foto postate da altri, reputandolo come un segno della considerazione di cui godono. Questi operai delle fabbriche si trovavano abitualmente ad essere quelli che 
"davano attenzione" nella loro vita quotidiana, dal momento che, in quanto gruppo svantaggiato, raramente avevano occasione di riceverne. Tuttavia, alcune giovani donne potevano essere preoccupate del fatto che nelle foto loro postate da altri potessero essere meno belle di quanto non fossero in foto postate da loro stesse. Questa considerazione risultava pure a Trinidad.

Il nostro sito brasiliano è sia amichevole sia aperto alla condivisione, ma possono esserci ancora diversi fattori che attenuano questa pratica. I teenager sono molto attenti a mettere foto online ed evitano quelle che recano informazioni sulle loro relazioni di status. Gli abitanti dei paesini solitamente sono attenti alle persone con cui si accompagnano perché ogni segno di prossimità (per esempio, un uomo e una donna che parlano per strada), può dare e spesso darà luogo a pettegolezzi in quanto i locali inferiranno che $\mathrm{i}$ due hanno una storia. Nel nostro sito inglese, c'era una radicale differenza tra le persone giovani, che raramente rimuovevano foto taggate a meno che non danneggiassero particolarmente la loro reputazione, e le persone più grandi, che sistematicamente rimuovevano questo tipo di immagini.

\section{Tema 4: il commercio}

Abbiamo indagato anche l'uso dei social media nell'ambito della sfera commerciale, cominciando con una domanda che chiedeva se le persone cliccassero sulle pubblicità.

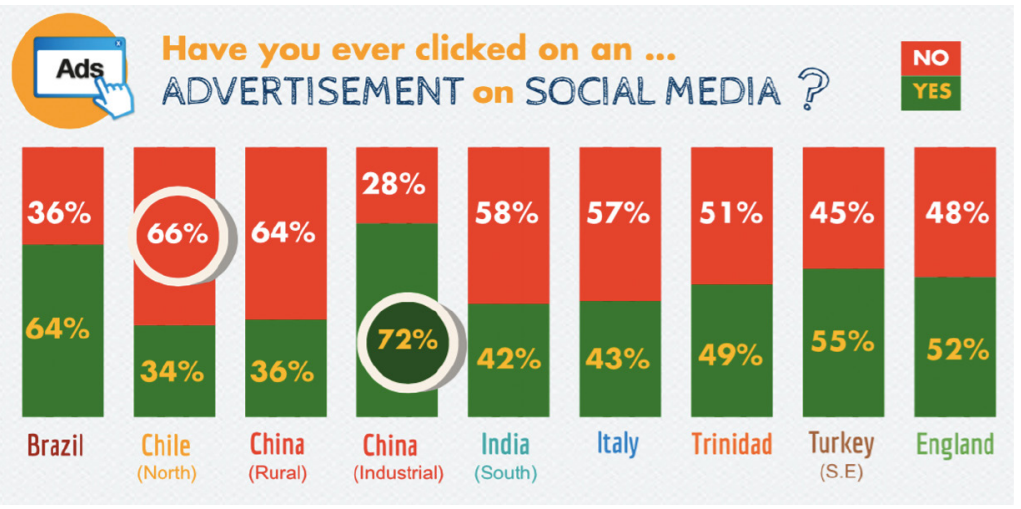

Fig. 4.15 Hai mai cliccato su una ... pubblicità sui social media? Distribuzione delle risposte alla domanda se gli utenti avessero mai cliccato su pubblicità sui social media 
Le risultanze del nostro sito del nord del Cile sono coerenti con una generale tendenza a evitare i consumi. La città di Alto Hospicio ha pochi negozi e anche poca pubblicità offline. Generalmente, il consumo, la fedeltà al marchio e anche l'ostentazione di mobilità verso l'alto qui sono considerati rozzi e superflui. Per quanto la città sia considerata 'marginale' non è 'povera'. Molti residenti beneficiano, dal punto di vista finanziario, del lavoro delle industrie connesse alle ricche risorse naturali della regione, come le miniere di rame. A guidare questa evidente mancanza di consumi è un senso di solidarietà nei confronti dei vicini e di adattamento a una forma di normalità modesta. Mentre gli individui molto spesso esprimono "mi piace" o "seguono" gli affari locali su Facebook, raramente consumano prodotti nazionali o internazionali del tipo di quelli che vengono pubblicizzati qui. Similmente Facebook non è usato come un sito per esprimere consumi evidenti. Del tutto diversa è la situazione dei nostri siti brasiliano e di Trinidad.

Se questo è un caso in cui non possiamo raggruppare i siti latino-americani, emerge anche una chiara discrasia tra i nostri siti della Cina rurale e industriale. Nel sito della Cina industriale è molto popolare condurre affari attraverso WeChat. Wang suggerisce che solo il 7\% circa di persone si impegna con intraprendenza nell'e.commerce, aprendo propri negozi on line o vendendo merci attraverso il proprio profilo sui social. Altri si impegnano in affari sui social in modo più passivo, soprattutto cliccando sulle pubblicità che vengono condivise con gli amici. Un'attività spesso menzionata e praticata connessa a un potenziale consumo è 'Jizan' ("raccogliendo 'mi piace"), una pratica su WeChat per ottenere regali o sconti. Anche i ristoranti locali usano Jizan per promuovere i loro affari. Le persone girano frequentemente promozioni di sconti sulle loro pagine personali di WeChat e spingono i loro contatti on line a esprimere "mi piace" sull'account WeChat del venditore ufficiale per ottenere merce scontata. Tutti incoraggiano le persone a cliccare sulla pubblicità dei loro profili sui social. Più in generale è appena il caso di notare che non aver mai cliccato su una pubblicità può non significare molto. Le nostre risultanze dicono di un impatto abbastanza limitato dei social sul commercio ${ }^{8}$.

Abbiamo poi cercato di vedere se i social media accrescevano il modo in cui le persone si influenzano reciprocamente riguardo al consumo, domandando se gli intervistati avessero mai acquistato qualcosa perché lo avevano visto a un amico sui social. Anche se il 30\% non è così significativo considerando che la domanda prevedeva che lo si fosse fatto almeno una volta, Miller fu 
sorpreso di scoprire che il sito inglese aveva la percentuale più alta. L'evidenza etnografica era del tutto diversa. Scoprì che gli informatori più adulti erano molto orgogliosi di dichiarare di non essere sensibili alla pubblicità, né online né offline. Forse essi avevano interpretato questa domanda nel senso di come venissero influenzati dagli amici in opposizione a come lo fossero dai social media. Il punto in Italia forse era non tanto che gli utenti si rifiutassero di essere influenzati dalle immagini dei social media quanto che quelle immagini riguardassero celebrità italiane piuttosto che quelle di loro amici.

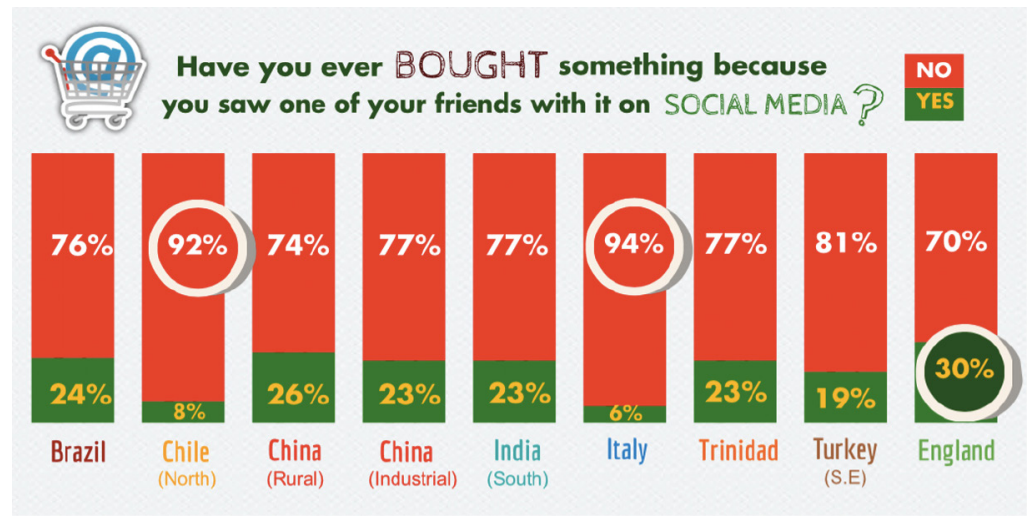

Fig. 4.16 Hai mai comprato qualcosa perché l'hai vista a un tuo amico sui social media? Distribuzione del comportamento riguardo agli acquisti

Abbiamo poi considerato se le persone facessero davvero soldi attraverso i social. Il risultato relativo al nostro sito nord cileno ancora una volta dovrebbe essere considerato in un contesto più ampio. Dal momento che questo è un luogo in cui le persone evitano ampiamente i contatti con la sfera commerciale formale, che è significativamente assente dalla loro città dati gli introiti provenienti dalle miniere, un uso informale dei social per vendite locali su piccola scala era diventato un sostituto di quel tipo di commercio formale. 


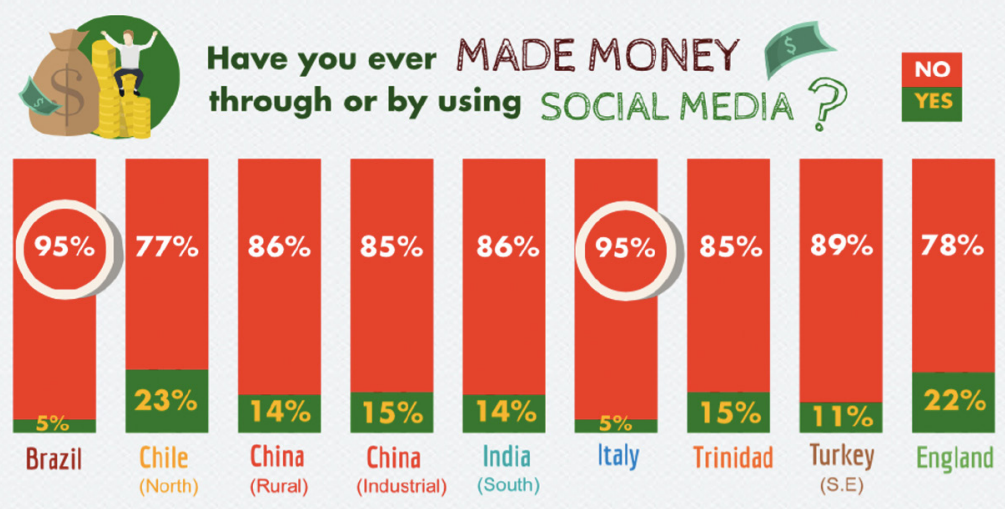

Fig. 4.17 Hai mai guadagnato soldi usando i social media? Distribuzione delle risposte alla domanda se gli utenti avessero mai guadagnato soldi attraverso $i$ social media

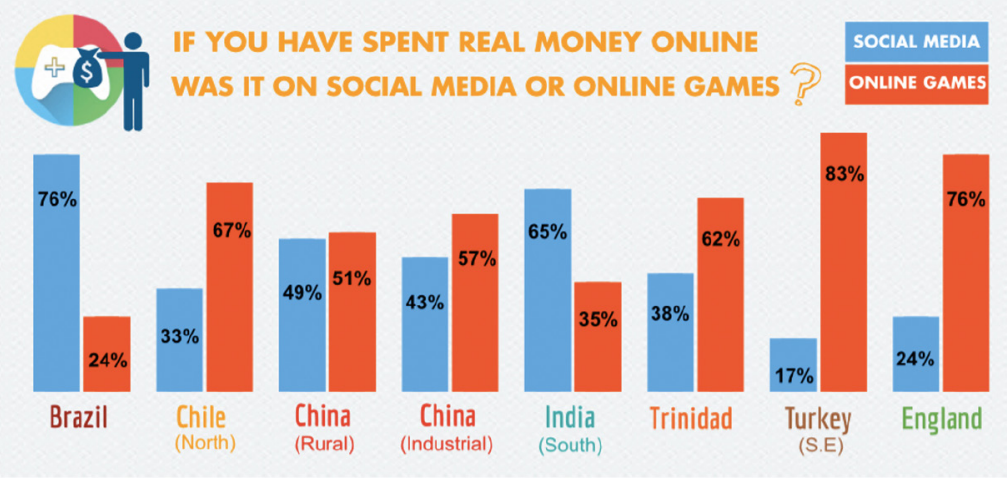

Fig. 4.18 Hai speso denaro reale sui social media o nei giochi on line?

Distribuzione di somme spese sui social media o nei giochi on line

Nella Cina industriale molti giochi sui social media erano pensati per incoraggiare la gente a spendere denaro per ottenere vantaggi al gioco. Questo viene discusso in dettaglio nei libri di McDonald e Wang 9. Questo può essere un fattore di richiamo soprattutto tra gli operai dato che l'aver raggiunto un alto livello nei giochi può diventare importante quando nella vita offline si ha uno status così basso.

Ancora una volta, il problema ha origine dal modo in cui le persone inter- 
pretavano la domanda. Nel sud dell'India le risposte a questa domanda riflettevano due categorie: 1) Spendere in piani tariffari a consumo per il traffico dati su internet, con l'intenzione di usarlo per attività sui social media; 2) spendere per comprare cose attraverso le pubblicità che compaiono sui social, che gli intervistati hanno considerato anche come 'spendere sui social media'.

Dal momento che i social media sono diventati un canale per il mercato locale, nazionale e globale, abbiamo investigato se le persone esprimono "mi piace" o "seguono" gli affari sui social.

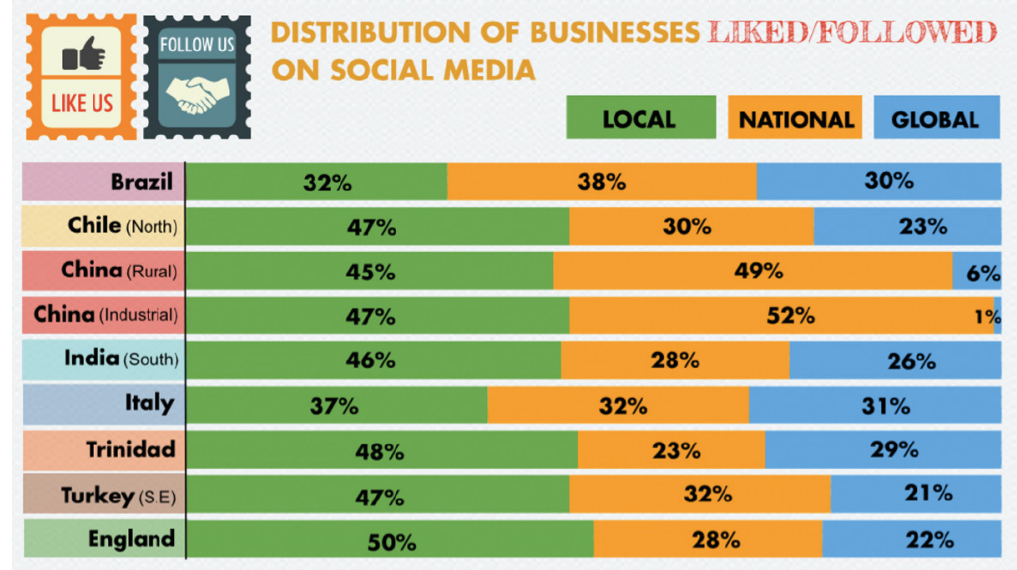

Fig. 4.19 Distribuzione di affari "Liked"|"Followed" sui Social Media

Ci sono diversi fattori da considerare dietro queste risposte, tra cui in primo luogo il fatto che esse rappresentano la distribuzione piuttosto che l'estensione di questo tipo di influenza. Prima di questo studio, Miller aveva già osservato, in riferimento a Trinidad, il grado di integrazione di Facebook negli affari locali ${ }^{10}$. Sinanan rilevava l'attività promozionale sui social per gli affari locali tipo i bar, la cui immagine e la cui reputazione erano particolarmente importanti. Inoltre, in riferimento al relativamente alto uso di commercio globale a Trinidad, coloro che avevano risposto di aver comprato beni in questo modo, generalmente si accordavano con i parenti residenti negli USA per farsi inviare gli articoli a Trinidad.

Non ci aspettavamo che i siti cinesi avessero link globali dal momento che la rete cinese è diretta essenzialmente all'interno. Qui Taobao è il sito di shopping on line più grande, insieme a WeChat. Entrambi si focalizzano ampiamente sul mercato interno. In altre aree le persone hanno interpretato 
la domanda in vari modi. I brasiliani possono considerare la Coca Cola come locale dal momento che è presente localmente anche se è un brand globale e la Nike può essere considerata nazionale in quanto associata a famosi calciatori brasiliani. Come abbiamo rilevato prima, nel sud dell'India le persone considerano che il denaro speso rispondendo alla pubblicità all'interno dei social sia speso all'interno delle stesse compagnie di social media. Una comprensione migliore della questione si ricava dal nostro lavoro qualitativo come descritto nel capitolo 6. L'analisi suggerisce che nella maggioranza dei siti l'impatto del mercato è limitato tranne per quegli affari in cui sono coinvolte relazioni personali, nel qual caso esso sembra essere importante.

\section{Tema 5: atteggiamenti verso i social media}

Oltre a conoscere il modo in cui le persone usano i social media, volevamo esplorare il loro atteggiamento verso i social e l'impatto di questi sui diversi aspetti delle loro vite. La nostra prima domanda su questo tema, presentata nella Fig. 4.20, riguarda i punti di vista delle persone sull'età ritenuta adeguata perché un bambino cominciasse a usare i social media. Le risposte vanno dai 13 anni della Cina rurale ai 17 anni del sud dell'India, ma confrontato con molte delle nostre domande il range è relativamente piccolo.

\section{At what $\mathrm{AOE}$}

do you feel it is acceptable for a child to start using social media?

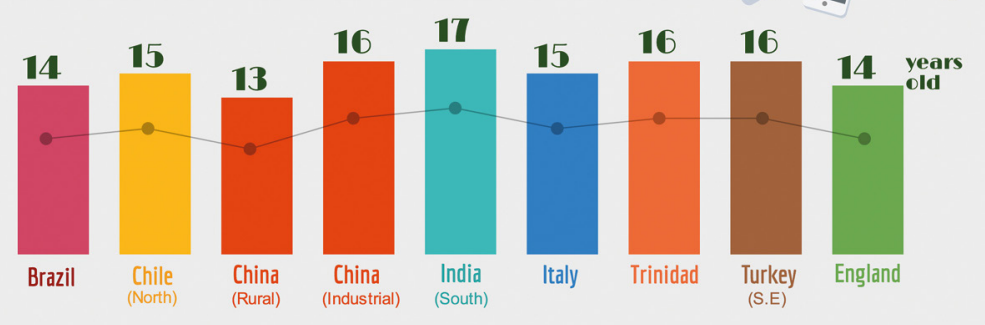

Fig. 4.20 A che età ritieni accettabile che un bambino cominci a usare $i$ social media? Distribuzione delle risposte alla domanda sull'età ritenuta appropriata per un bambino all'uso dei social media 
Abbiamo poi chiesto se le persone usassero i social media nel loro posto di lavoro e/o o nei luoghi di istruzione.

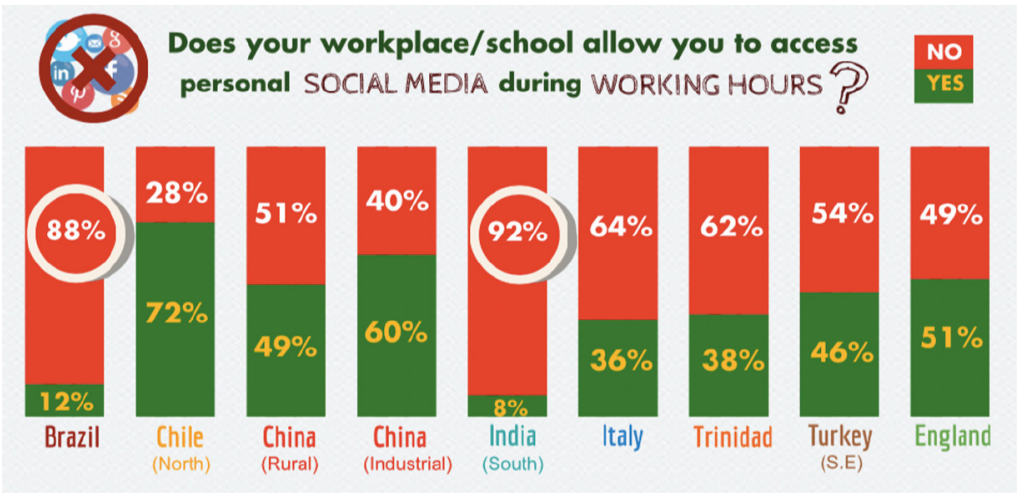

Fig. 4.21 Il tuo posto di lavorolla tua scuola ti consente di accedere ai tuoi social media durante le ore di impegno? Distribuzione dell'accesso ai social media durante l'impegno lavorativo (posto di lavoro/scuola)

La Fig. 4.21 riguarda la restrizione/proibizione di usare i social sul posto di lavoro o presso le istituzioni preposte all'istruzione nel sito brasiliano e dell'India meridionale. Il fatto non è sorprendente dal momento che nel sito brasiliano la maggior parte lavora in alberghi, come cameriere o addetto alle pulizie, sotto stretta supervisione delle loro mansioni. Tuttavia, la nostra ricerca qualitativa relativa al modo in cui, nella prassi, le persone riuscivano ad aggirare queste restrizioni nell' uso dei social, rivela modelli molto diversi. Ciò era particolarmente vero in India dove i moderni posti di lavoro e le istituzioni che si occupano di istruzione erano molto restrittivi. Ciò nonostante, in entrambi i posti gli smartphone rendono particolarmente difficile regolare e far rispettare questo tipo di restrizioni.

Abbiamo poi chiesto se le persone ritenevano che i social media fossero una buona cosa per l'istruzione. Questa domanda sarà attentamente considerata nel prossimo capitolo perché si è trattato di una questione controversa in molti dei nostri siti. Qui vedremo come nel sud dell'India le scuole con ingressi a più basso reddito incoraggiavano l'uso dei social come strumento per l'educazione, mentre quelle frequentate dalle classi più alte lo consideravano una distrazione e dunque da bandire. 


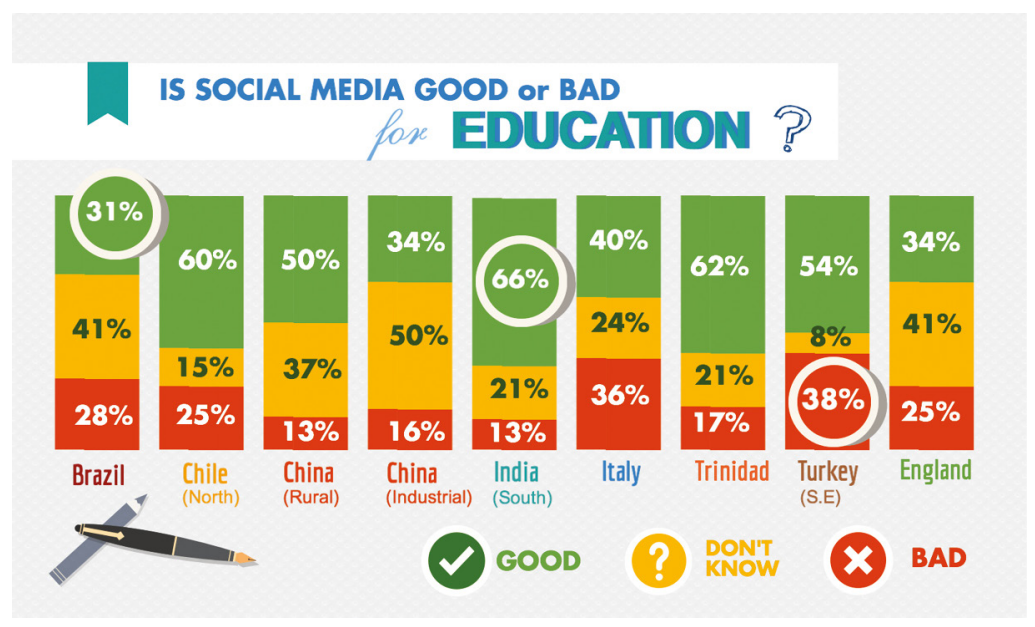

Fig.4.22 I social media sono buoni o cattivi per l'istruzione? Distribuzione delle risposte alla domanda se i social media abbiano effetti buoni o cattivi sull'educazione

Una domanda analoga ha riguardato l'impatto dei social media sul lavoro. Come illustrato nella Fig. 4.23, le risposte dei brasiliani echeggiavano la percezione da parte dei manager che considerano i social una distrazione nel posto di lavoro. Tuttavia, anche qui i manager riconoscevano che i social potessero avere pure un effetto positivo. Per esempio, quando una collaboratrice domestica vede che il suo datore di lavoro ha bisogno di un professionista per risolvere un problema elettrico, usa WhatsApp per contattare un amico o un parente per fare il lavoro. Molti sembrano considerare l'impatto positivo dei social media come superamento della loro natura di svago. 


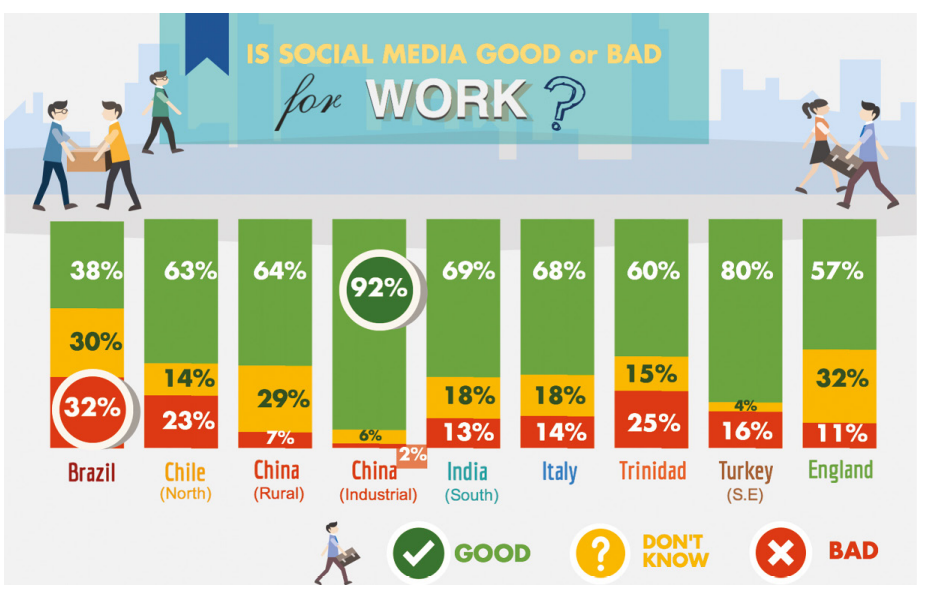

Fig. 4.23 I social media sono buoni o cattivi per il lavoro? Distribuzione delle risposte alla domanda se $i$ social media abbiano effetti buoni o cattivi sul lavoro

La prospettiva successiva riguardava una indagine più generale relativa al fatto di avvertire eventualmente i social media come un peso. Molte di queste domande, così come i titoli di diversi capitoli di questo libro, piuttosto che esprimere soltanto un interesse accademico, riflettono il nostro desiderio di rispondere alle domande comuni che vengono poste a noi. In questo caso la risposta è interessante soprattutto per il contrasto rispetto alla rappresentazione dominante dei social nell'editoria corrente.

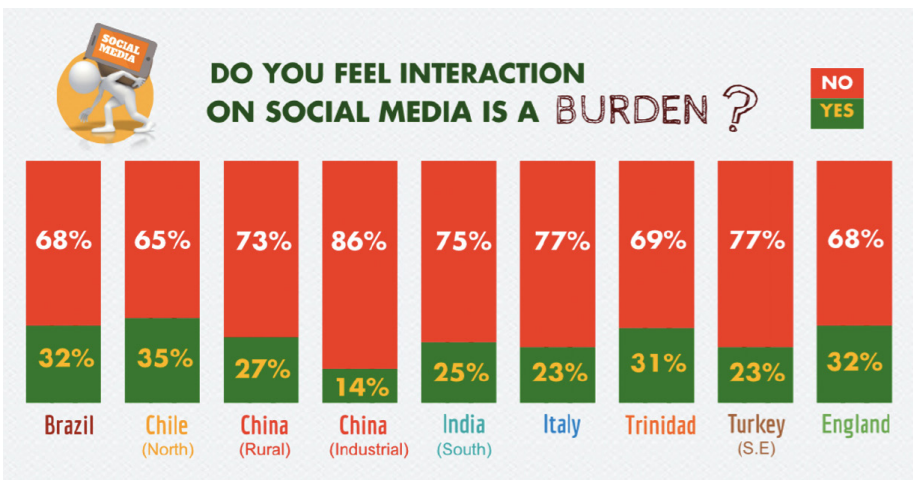

Fig. 4.24 Ritieni che l'interazione sui social media sia un peso? Distribuzione delle risposte alla domanda se gli utenti ritengano un peso l'interazione sui social media 
La domanda successiva ha a che fare pure con discussioni correnti nei giornali e altrove. Fig. 4.25

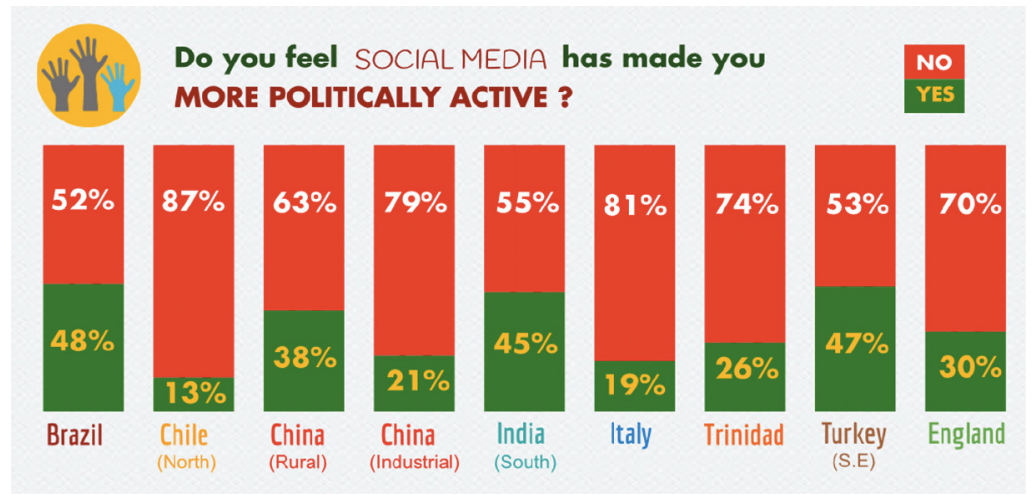

Fig. 4.25 Ritieni che i social media ti abbiano reso politicamente più attivo?

Distribuzione delle risposte alla domanda se i social media abbiano reso gli utenti politicamente più attivi

Forse in questi risultati pesa una componente temporale specifica. Le due risposte positive più alte si riferiscono al sito brasiliano e a quello turco. In entrambi i paesi durante la ricerca ci sono state proteste popolari di alto profilo: in Brasile, contro il presidente Dilma Rousseff e in Turchia a Gezi Park e l'uso dei social media era strettamente connesso a questi eventi. Vale la pena notare che, nella pratica, Spyer rilevò che in quei giorni nei social media locali non c'erano stati commenti diretti e, pertanto, i risultati di questa indagine sembrano essere più un'associazione che ha lasciato la sua impressione piuttosto che una riflessione su ciò che le persone fanno davvero. $\mathrm{Nel}$ sud dell'India era un periodo in cui le persone cominciavano a rendersi conto che alcune questioni (come l'abuso delle donne) erano diventate più evidenti in particolare attraverso i social media e da qui assumevano una maggiore visibilità sui mass media tradizionali quali giornali e televisione.

Infine, l'inchiesta riecheggia un'altra domanda molto generale (Fig. 4.26) che ci viene posta fuori dall'accademia e su cui riflettiamo nel Capitolo 13: se i social media abbiano lasciato i rispondenti più felici, meno felici o se non c'è stata alcuna differenza. 


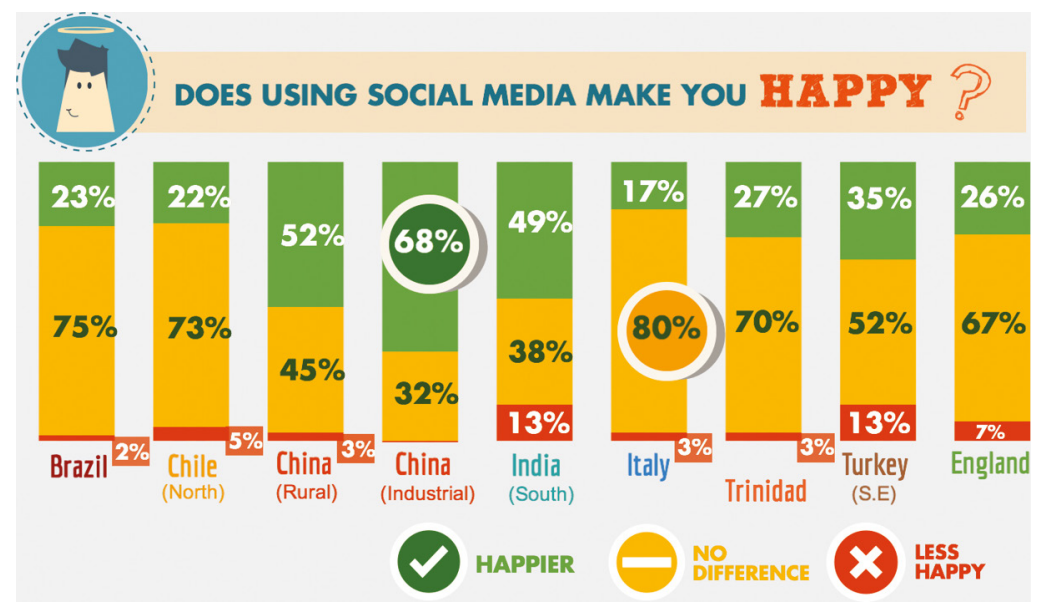

Fig. 4.26 L'uso dei social media ti rende felice? Distribuzione delle risposte alla domanda se l'uso dei social media renda gli utenti piu felici

Quest'ultima domanda, più o meno come la prima, è stata una che davvero aveva un senso ponendola in relazione con la ricerca qualitativa. Nei siti più conservatori del sud dell'India e della Turchia sudorientale abbiamo rilevato che i social media rappresentavano la differenza più grande nelle vite della gente perché aprivano a nuove possibilità. Gli operai cinesi, d'altro canto, diventavano più dipendenti dai social per la realizzazione delle loro aspirazioni e per compensare l'assoluto grado di infelicità delle loro condizioni offline. Tuttavia, il risultato più chiaro riguarda la quasi assenza di sentimenti negativi in generale, se si fa una comparazione con i report giornalistici (si veda il Capitolo 13).

\section{Conclusioni}

Può essere raro che un insieme di dati venga pubblicato con così tante precisazioni e autocritiche. Per quasi tutte le domande di questa indagine almeno uno dei ricercatori ha suggerito che il risultato dovesse essere letto alla luce del contesto della ricerca sul campo; le risposte possono dipendere dal modo in cui le persone hanno interpretato le domande piuttosto che riflettere il loro reale comportamento. Come abbiamo osservato all'inizio di questo ca- 
pitolo, non ci siamo pentiti di aver condotto questi questionari e di aver tentato di comprenderne i risultati. La gamma delle risposte a molte delle domande sostiene anche uno dei punti centrali dell'intero progetto, ossia che una presunta estrapolazione di dati quantitativi da un posto a un altro è problematica. Questi risultati forniscono almeno un'impressione visiva della dimensione delle diversità regionali nell'uso dei social media.

L'altra ricompensa per questo nostro esercizio è il fatto che è emerso che molte domande erano produttive. I ricercatori hanno dovuto domandarsi perché le persone rispondevano nel modo in cui rispondevano, cosa particolarmente utile quando i risultati non si accordavano con le risultanze della ricerca sul campo di 15 mesi. I risultati della ricerca quantitativa ci hanno spinti a guardare più in profondità la nostra comprensione secondo l'approccio qualitativo. Un esempio è stato quanto ha scoperto Spyer riguardo al sito brasiliano in cui le risposte relative al fatto di aggiungere amici significavano che le persone si erano già consultate con altri prima di farlo e non che avevano chiesto il permesso per farlo.

Il materiale di questo capitolo è tratto da un singolo questionario con 1199 persone che hanno risposto da tutti e nove i siti della ricerca. Abbiamo resistito a ogni tentazione di cercare correlazioni. Con questo tipo di materiale esse sono in ogni caso problematiche, ma lo sarebbero state ancora di più considerato l'estensione del range per età, genere e condizione sociale dei rispondenti. Speriamo che dalla nostra discussione sia emerso con chiarezza perché rimaniamo cauti nell'uso dei dati quantitativi. Nessuno dei punti e dei problemi che abbiamo segnalato in relazione all'interpretazione di queste tabelle ha a che fare con le piccole dimensioni del questionario. Sarebbero rimasti esattamente gli stessi anche se avessimo posto le questioni a un numero di persone dieci volte più grande. La nostra enfasi generale sulla maggiore autorevolezza e affidabilità delle risultanze qualitative, pertanto, non riguarda il fatto che non portiamo mai avanti inchieste di tipo quantitativo, ma è proprio perché qualche volta lo facciamo.

\section{Background dello studio}

In etnografia i questionari sono comunemente usati ${ }^{11}$. Come accade tipicamente nell'esercizio dell'antropologia, coloro che li usano sperano di assolvere altri compiti oltre a quello di generare dati statistici. Durante i 15 mesi di ricerca sul campo abbiamo condotto due diverse inchieste basate su 
questionari $^{12}$ (li chiameremo Questionario A e Questionario B), comuni a tutti e nove i nostri siti. Oltre a queste inchieste comuni, ognuno di noi ha condotto la propria inchiesta indipendente basata su un questionario, specificamente ideata per soddisfare il proprio sito. Per esempio, in Inghilterra abbiamo intrapreso un'inchiesta con 2496 studenti di scuola; in Italia con 750 studenti tra i 16 e i 18 anni; nella Cina industriale con 238 migranti rurali. I risultati di molte di queste inchieste sono inclusi in questo capitolo.

Per molti di noi, questi questionari funzionavano da strumenti per la costruzione di rapporti con potenziali informatori. Spesso, iniziare con un questionario pone i nostri informatori a proprio agio e fornisce un mezzo per riuscire a conoscerli in modo meno formale successivamente. In pochi siti le persone si aspettavano che il ricercatore ponesse loro domande dirette e questo li rassicurava che si trattasse di veri e propri ricercatori.

Oltre a raccogliere numeri, lo scopo primario del Questionario A era quello di includere un campione rappresentativo di persone di diverse categorie di reddito per ciascuno dei siti. L'indagine così ci aiutava ad assicurarci sin dall'inizio un impegno attivo con persone appartenenti all'intera gamma di categorie di reddito; era utile per spingerci a un impegno più rappresentativo con la nostra popolazione. Il Questionario A veniva somministrato ad almeno 100 persone per ciascun sito, incluse quelle che non facevano uso di social media. Dal momento che veniva somministrato all'inizio del fieldwork non avevamo ancora risolto come porre ogni domanda in un modo culturalmente appropriato per tutti i siti. Un confronto diretto di questi risultati sarebbe perciò fuorviante. Ogni ricercatore invece ha usato questi risultati all'interno del proprio studio etnografico individuale ${ }^{13}$.

Questo capitolo, invece, si basa sul Questionario B, la nostra seconda indagine, diretta interamente agli utenti dei social media. Questo esercizio è stato condotto durante gli ultimi tre mesi della nostra ricerca sul campo. Dopo un anno sul campo, speravamo di creare un insieme di domande comuni che fossero comparabili in modo diretto ${ }^{14}$. Abbiamo affrontato una sfida particolare con i siti cinesi rurale e industriale che usano le piattaforme dei social media in modo del tutto diverso rispetto agli altri siti, e queste hanno diverse funzionalità. Questo capitolo include così solo quelle domande che riteniamo possano essere ragionevolmente comparabili attraverso i nove siti. Volevamo anche una certa parità di genere, anche se di fatto essa varia dal $67 \%$ delle donne che hanno risposto al questionario, nel sito inglese, al 61\% degli uomini per la Cina industriale. In generale, il 55\% erano donne e il 45\% uomini (Fig. 4.27). 
Come il mondo ha cambiato i social media

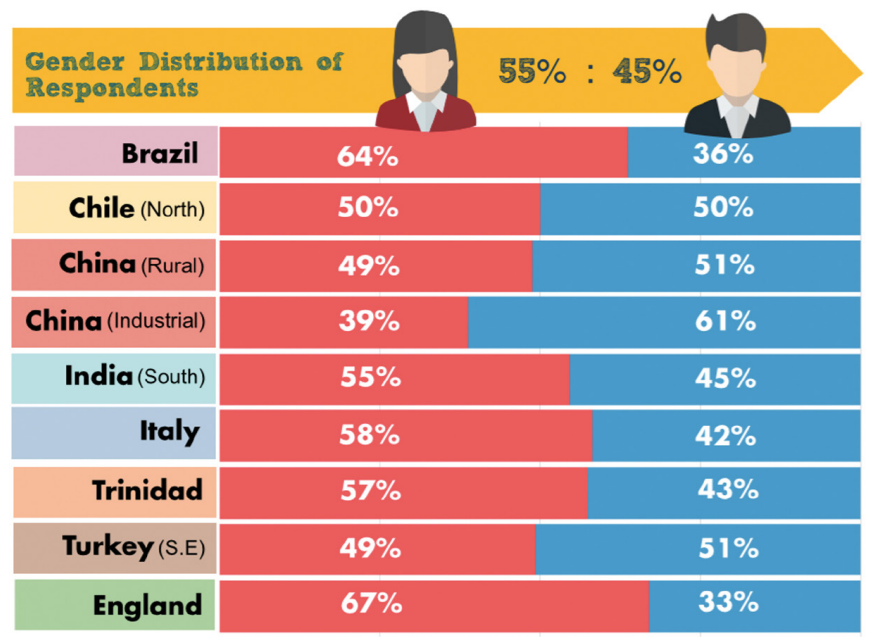

Fig. 4.27 Distribuzione delle risposte in base al genere.

C'era maggiore variabilità riguardo all'età, dal momento che l'uso dei social media nei nostri siti europei si è avviato con le generazioni più grandi (come si mostra nella Tabella 4.5). In generale circa il 73\% di coloro che hanno risposto era sotto i 29 anni.

\begin{tabular}{|c|c|c|c|c|c|c|c|c|}
\hline & & \multicolumn{4}{|c|}{$\begin{array}{l}\text { AGE DISTRIBUTION } \\
\text { OF RESPONDENTS }\end{array}$} & & & \\
\hline c) & $13-19$ & $20-29$ & $30-39$ & 40-49 & $50-59$ & $60-69$ & $70-79$ & $80-89$ \\
\hline Brazil & $49 \%$ & $35 \%$ & $12 \%$ & $2 \%$ & $3 \%$ & $0 \%$ & $0 \%$ & $0 \%$ \\
\hline Chile (North) & $24 \%$ & $51 \%$ & $14 \%$ & $9 \%$ & $2 \%$ & $0 \%$ & $0 \%$ & $0 \%$ \\
\hline China (Rural) & $57 \%$ & $21 \%$ & $15 \%$ & $6 \%$ & $1 \%$ & $0 \%$ & $0 \%$ & $0 \%$ \\
\hline China (Industrial) & $9 \%$ & $57 \%$ & $22 \%$ & $6 \%$ & $6 \%$ & $0 \%$ & $0 \%$ & $0 \%$ \\
\hline India (South) & $18 \%$ & $64 \%$ & $7 \%$ & $7 \%$ & $3 \%$ & $1 \%$ & $0 \%$ & $0 \%$ \\
\hline Italy & $24 \%$ & $29 \%$ & $18 \%$ & $20 \%$ & $6 \%$ & $2 \%$ & $1 \%$ & $0 \%$ \\
\hline Trinidad & $39 \%$ & $47 \%$ & $10 \%$ & $2 \%$ & $2 \%$ & $0 \%$ & $0 \%$ & $1 \%$ \\
\hline Turkey (S.E) & $30 \%$ & $42 \%$ & $20 \%$ & $8 \%$ & $1 \%$ & $0 \%$ & $0 \%$ & $0 \%$ \\
\hline England & $19 \%$ & $32 \%$ & $24 \%$ & $8 \%$ & $10 \%$ & $6 \%$ & $1 \%$ & $0 \%$ \\
\hline
\end{tabular}

Tavola 4.5 Distribuzione delle risposte in base all'età. 
I dieci temi chiave 



\section{Capitolo 5 \\ Educazione e giovani}

Se, nello studio dei social media, ci sono due argomenti che si stagliano per la notevole attenzione di cui sono stati fatti oggetto, questi sono la politica (Capitolo 10) e l'educazione. Quest'ultimo gode di particolare interesse dal momento che i social in sé sono strettamente associati ai giovani e considerati da molti come il 'luogo' in cui i giovani trascorrono la maggior parte del loro tempo. Circola anche una significativa incertezza e preoccupazione sul più ampio impatto dei social media sull'educazione e il benessere delle giovani generazioni. C'è chi ritiene che i social stiano distruggendo il sistema dell' istruzione e porteranno a un sensibile abbassamento dei voti, che può essere risolto soltanto proibendo l'accesso ai cellulari e ad altre tecnologie di comunicazione e informazione (ICT). Altri vedono i social media come un potenziale per ridare nuova linfa all'esperienza educativa e pensano che forzando l'educazione formale nella direzione di modalità di apprendimento informali e interattive, l'uso di queste tecnologie porterà inevitabilmente un beneficio a tutti gli interessati.

Questo capitolo si terrà alla larga da questo tipo di posizioni polarizzate per concentrarsi invece sulle reali pratiche dei social così come si articolano nei nostri siti di ricerca. In primo luogo, esaminerà la distinzione che ancora persiste tra modalità di apprendimento formale e informale, mostrando come i social media stanno rappresentando una sfida a questi confini in molti dei nostri siti. In alcuni luoghi essi stanno facilitando un indietreggiamento dall'apprendimento formale.

Il capitolo dedica anche una maggiore attenzione al modo in cui i social media stanno rimodellando le relazioni chiave implicate nella dimensione educativa: tra studenti e studenti, tra studenti e docenti, tra docenti e genitori. L'esame di ciascuna di queste relazioni rivela temi chiave, soprattutto familiarità e conflitto, sorveglianza e impegno, mediando la comprensione dei doveri reciproci. Tutto ciò fa luce sull'importanza sociale dell'apprendimento, sempre più condotto attraverso lo spazio on line. 


\section{Letteratura e ricerche precedenti}

Come è stato rilevato nelle rassegne dei nostri sudi accademici sui social media, le ricerche sull'educazione spesso sono esemplari. Si tratta di un ambito in cui gli studiosi del sociale si sono adoperati per produrre ricerche equilibrate e sistematiche per informare il pubblico dibattito. Gran parte di questa ricerca è influenzata dal concetto di 'alfabetizzazione mediatica' ('educazione ai media'). Questo progetto politico incentrato sul bambino pone l'enfasi sull'opportunità di dotare le persone di abilità che le rendano capaci di analizzare criticamente e di produrre con competenza messaggi mediatici. Così facendo, il progetto sottolinea una competenza di produzione, di linguaggio, di rappresentazione e di audience ${ }^{1}$.

Un esempio è stato il progetto sull'uso dei nuovi media dei teenager americani, condotto da Ito e i suoi collaboratori ${ }^{2}$. Gli autori mostrano come i teenager usano i nuovi media per creare opportunità di amicizia motivata dall'apprendimento tra pari, e favorendo l'alfabetizzazione mediatica. Gli autori sostengono che questo funziona meglio in un ambiente generale che ha a che fare con il "passare il tempo", con il "bighellonare", insomma con il dare ai giovani un accesso parziale a una più ampia sfera pubblica. Questo tema è accolto qui, ritendendo che i social media diano un contributo positivo se si considera l'apprendimento in senso più generale, piuttosto che nel senso ristretto di educazione formale ${ }^{3}$.

In questo studio i social media sono considerati entro un più ampio contesto di nuovi media. L'antropologo Lange ${ }^{4}$, per esempio, ha esaminato i modi creativi in cui i giovani usano YouTube attraverso la produzione di video effimeri, spontanei e 'personalmente espressivi' realizzati in modo collaborativo con i pari. Michael Wesch ${ }^{5}$ conduce uno studio etnografico di lungo termine sui video blogging; pone l'accento sull'apprendimento, la più ampia interattività, le nuove forme di comunità ${ }^{6}$ e sulle competenze e abilità acquisite attraverso questo processo. Il concetto di "apprendimento connesso", che mette in risalto l'interazione continuata e l'apprendimento 'attivo', rappresenta più in generale la punta avanzata della teoria dell'educazione? Questi studi dimostrano anche che le tanto spesso denigrate 'identità geek' sono sempre più centrali nelle giovani generazioni che altrimenti considereremmo parte del mainstream ${ }^{8}$.

Sia boyd ${ }^{9}$ che Clark ${ }^{10}$ dimostrano come i problemi connessi all'uso dei social media siano ingiustamente attribuiti al comportamento dei giovani, mentre fare luce sul comportamento dei genitori nella gestione e nell'uso 
dei social è un fattore di pari - se non più grande - influenza. Capire che le pressioni dei genitori sui giovani spesso sono contraddittorie, illogiche e inutili è un importante complemento sul focus ristretto sugli stessi giovani. Nel nostro sito inglese è la sorveglianza dei genitori piuttosto che quella delle compagnie di social media a preoccupare i bambini. L'importanza di prestare attenzione a questo contesto più ampio emerge in un libro etnografico di prossima pubblicazione di Livingston e collaboratori ${ }^{11}$. Basato su un'etnografia di una classe scolastica, in Inghilterra, include anche momenti trascorsi con questi bambini nelle loro case.

Per boyd e Clark questo è particolarmente importante dal momento che i teenager vogliono (e hanno anche bisogno di) un certo grado di autonomia dai genitori e altre figure più grandi per sviluppare una propria esperienza di gestione delle interazioni sociali, soprattutto con i loro pari. In questo senso, l'alfabetizzazione mediatica è anche una forma di alfabetizzazione sociale che potenzialmente è facilitata dai social media, a patto che i genitori diano loro lo spazio per svilupparla.

Clark sottolinea la dimensione classe, soggiacente a questa condizione. In linea di massima sono i genitori meglio istruiti e benestanti ad apprezzare il fatto che possedere queste abilità nella gestione della comunicazione digitale potenzierà le chance di vita della loro prole, accentuando così le precedenti differenze di classe. Altri notano come i social media e i nuovi media siano diventati simboli di cosmopolitismo e anche uno strumento per incrementarlo ${ }^{12}$. Questo è particolarmente rilevante in molti dei posti piccoli che costituiscono i nostri siti di ricerca. Qui nuove tecnologie di comunicazione e informazione (ICT) possono ampliare il divario tra questi nuovi cosmopoliti e famiglie più povere e molto meno istruite, che finiscono con il trovarsi di fronte a un'ulteriore barriera all'inclusione sociale.

Altri studiosi hanno condotto ricerche al di là della relazione genitori-figli per indagare l'impatto di gruppi quali governi e sistemi educativi che hanno autorità e rappresentano infrastrutture, inclusi il sistema normativo e scolastico. Questo aspetto rende il lavoro di Livingstone e dei suoi colleghi così importante. Hanno condotto indagini estensive sul livello di uso dei social media nelle scuole e da parte dei bambini, presentando i loro risultati come una guida equilibrata, di buon senso e di facile comprensione che può confluire direttamente nell'ambito della politica dell'istruzione, della normativa e della protezione del bambino. Questo, a sua volta, consente interventi maggiormente informati e sensibili. La ricerca di Livingston e colleghi ri- 
sponde anche alle preoccupazioni dei genitori riguardo ai loro figli e alla preoccupazione di Clark e boyd riguardo al comportamento dei genitori. Essi raccomandano una delicata negoziazione tra il rispetto dell'autonomia delle giovani generazioni e l'attenzione nei confronti del pericolo che per i giovani rappresenta l'essere esposti ai nuovi social media.

Non abbiamo alcuna intenzione di sminuire il contributo di queste nuove ricerche in ambito accademico, basate inusualmente su studi etnografici qualitativi estensivi dello stesso tipo di quelli di cui noi siamo fautori (nel caso di Livingston la ricerca è supportata da comparazioni estensive in prospettiva interculturale $)^{13}$. Il contributo di questo capitolo è davvero trasversale, dal momento che riconosce che per la maggior parte dei bambini del mondo l'esperienza sia scolastica sia riguardo al rapporto con i genitori è molto diversa nei siti oggetto di questo studio, dal Nord America all'Europa. L'enfasi sul contesto che essi implicano, diverrà persino più evidente nel momento in cui volgiamo la nostra attenzione a situazioni molto diverse, come il Brasile, la Cina, l'India, la Turchia - anche se troveremo differenze marcate tra siti apparentemente simili quali possono essere un paesino inglese o una cittadina del sud Italia.

\section{Dall'apprendimento 'formale' a quello 'informale': attenuare le debolezze percepite nell'ambito dell'istruzione}

Nei nostri siti di ricerca economicamente meno sviluppati (Brasile, Cina, e le componenti rurali del sud dell'India) ${ }^{14}$, i genitori sono afflitti da marcate disuguaglianze finanziarie. Essi hanno espresso spesso una generale frustrazione per le carenze delle scuole locali nel fornire adeguatamente agli studenti le abilità e le competenze richieste per il successo nell'istruzione formale, nel lavoro e più in generale nella vita. In risposta, molti giovani si sono appropriati in modo creativo dei social media per acquisire strumenti supplementari di apprendimento e di rete per la loro educazione. Talvolta, li hanno considerati più utili e rilevanti per le loro vite.

Nel sito brasiliano Spyer ha rilevato che i genitori consideravano l'interesse dei figli per il computer come un fatto positivo per il loro futuro, ritenendo che li avrebbe aiutati a diventare meglio informati e più connessi con il mondo. Al contrario, gli insegnanti vedevano i social media come la "rete cattiva", perché distraeva i bambini, aveva un impatto deleterio sui loro 
studi, minava l'autorità professionale e causava disturbo in classe. Google, d'altro canto, veniva considerato come la "rete buona" in quanto fonte affidabile per la conoscenza. Gli studenti consideravano i social media un aiuto per migliorare la loro capacità di leggere e scrivere, in parte anche per le loro criticità nello spelling tra e con i pari, cosa che non veniva riconosciuta né dai genitori né dagli insegnanti. I social media inoltre davano una certa autonomia dai genitori, che spesso avevano una capacità di lettura significativamente più scarsa della loro.

Il sito del sud dell'India ha mappato questa varietà di atteggiamenti riguardo alla classe sociale e al tipo di istruzione scolastica ricevuta. Venkatraman ha rilevato una consistente variazione tra le scuole medie (studenti tra gli 11 e i 15 anni) sia di contesto sia di qualità dell'insegnamento. I ragazzi di famiglie ricche e di classe media che frequentano scuole private e internazionali tendevano a usare i social media con un livello comparativamente alto di monitoraggio da parte dei loro genitori: il risultato dell'interesse da parte dei genitori riguardava l'effetto di questo uso sul raggiungimento degli obiettivi nell'istruzione. Di contro, i figli di famiglie più povere che frequentavano scuole statali generalmente avevano un atteggiamento molto più positivo; $\mathrm{i}$ loro genitori percepivano i siti di social media come educativi in se stessi, a prescindere dal modo in cui venissero usati.

Similmente, i genitori di classe più bassa, i cui figli frequentavano scuole prive di supporti tecnologici ITC, tendevano a incoraggiarli particolarmente all'uso dei social media, sperando che questo avrebbe fornito loro abilità tecnologiche e, pertanto, possibilità di impiego nell'enorme parco tecnologico sviluppato nell'area di riferimento. Alle donne nubili con basso reddito era proibito possedere un proprio cellulare, soprattutto per il timore che l'uso potesse portarle a sposarsi fuori dalle prescrizioni di casta. Tuttavia, molte giovani donne aggiravano queste restrizioni, accedendo ai social media per mezzo dei telefoni dei colleghi di scuola o prendendo un lavoro che richiedeva che si possedesse un cellulare con accesso a internet.

Nella Cina industriale Wang ha scoperto che i migranti operai delle fabbriche mostravano scarso interesse al buon andamento dell'educazione formale o alla prosecuzione dell'istruzione di livello superiore dei loro figli, in netto contrasto con gli standard generali dell'educazione in Cina ${ }^{15}$, che sottolinea la radicale differenza di sistemi di valore di questi gruppi. I lavoratori delle industrie consideravano inevitabile il fatto che i loro figli si ritirassero dalla scuola per fare un lavoro manuale. Cosa che veniva aggravata 
dalla segregazione di questi bambini in scuole separate rispetto ai residenti locali. Wang ha osservato che, visto il disimpegno dall'educazione formale, i lavoratori delle industrie e i loro figli spesso usavano i social media per leggere ampi articoli su argomenti considerati utili, per esempio auto-aiuto, nutrizione, salute e consigli finanziari. Essi apparivano poco interessati a condividerli con i loro amici o a comunicarli. Li archiviavano invece postandoli sui propri profili in modo che essi stessi potessero ritornare a vederli successivamente. Questa appropriazione dei social media come stoccaggio e riesame di conoscenza è esemplificativo del modo in cui le popolazioni convertono specifiche piattaforme in strumenti di apprendimento.

La situazione relativa al sito della Cina rurale era piuttosto all'opposto. Qui i genitori attribuivano grande importanza all'educazione dei loro figli, ritenendo che i risultati accademici avrebbero aiutato i loro figli ad avere una vita sicura e agiata. In questo contesto, i social media venivano concepiti (non solo dai genitori, ma dagli stessi studenti) come forieri di un impatto negativo sulla progressione e l'apprendimento nel processo formativo. Gli studenti trascorrevano ogni giorno molte ore a scuola. Durante le vacanze estive spesso seguivano corsi privati costosi in città. I genitori volevano che i loro figli arrivassero a frequentare l'università, ma avevano poca cognizione su quale tipo di competenze fosse necessario agli studenti per superare gli esami o su come fosse realmente la vita universitaria.

Tutto ciò costituiva delle restrizioni significative a dispetto delle quali gli studenti trovavano modi di accedere ai social media. Essi facevano un uso estensivo di "QQ Groups", con messaggi di gruppo istantanei che corrispondevano fisicamente ai gruppi classe. Al di là della socializzazione in forma continua con i compagni di classe fuori dall'orario scolastico, gli studenti erano soliti condividere le risposte dei compiti per casa e cercare aiuto da altri compagni di classe. Chiedere aiuto tra pari era particolarmente importante dato che i genitori erano spesso privi delle conoscenze pertinenti rispetto ai compiti per casa e incontrarsi tra compagni fuori dall'orario scolastico in questa area rurale era spesso difficile.

Se i siti del Brasile, della Cina e del sud dell'India presentano delle disparità di profilo quanto alle condizioni economiche, alcune famiglie nel sito del sud Italia, comparativamente più omogeneo, hanno mostrato analoghe riserve riguardo alla capacità di scuole e università di fornire in modo esaustivo ai loro figli gli strumenti utili per il mercato del lavoro. Nicolescu rileva che mentre i docenti generalmente proibivano l'uso dei cellulari in classe, 
molti studenti, soprattutto quelli delle scuole professionali, si sentivano in diritto di usare questi dispositivi in classe. Piuttosto che l'educazione formale, per trovare lavoro essi ritenevano molto più utili proprio le forme di amicizia e solidarietà alimentate dai social media. Con il risultato che gli studenti consideravano i docenti come l'unico gruppo che non li supportava nell'uso dei social media.

A Trinidad la condizione economica era un altro fattore di differenziazione degli atteggiamenti nei confronti dei social media. Le famiglie facoltose preferiscono iscrivere i loro figli presso prestigiose scuole private e pagare lezioni di doposcuola. Questo tipo di studenti solitamente fa uso di internet per condurre ricerche e completare i compiti per casa. Le famiglie a basso reddito, invece, i cui adulti avevano pochissima educazione formale, ponevano scarsa enfasi sull'uso dei social media da parte dei loro figli. Similmente, nel sito della Turchia sudorientale, le famiglie agiate e d'élite favoriscono l'uso dei social media in quanto vantaggioso per l'istruzione.

Per riassumere, questo studio rivela che in alcuni dei nostri siti sono le famiglie ricche ad avere una visione positiva dell'uso dei social media per l'istruzione. Più comunemente, le persone che valutano positivamente l'educazione formale e ci credono si preoccupano che i social media possano distogliere da essa mentre coloro che ne sono delusi sperano che i social media possano fornire un efficace accesso all'educazione informale. Questi ultimi sono in genere le famiglie a basso reddito. Altri fattori riguardano anche se i social media siano visti come strumento di mobilità sociale o meno, e se il loro uso sia considerato come accessorio o alternativo all'educazione formale. Tra coloro che sono più benestanti ci sono alcuni che hanno fiducia nel fatto che i figli facciano un uso positivo dei social. Per esempio, nel sito inglese molte scuole secondarie (studenti da 11 a 18 anni) settano account di Twitter finalizzati a informare studenti di specifici anni di corso sulle attività collegate alla scuola.

In tutti questi casi, tuttavia, un ampio uso di ICT ha trasformato la relazione tra educazione formale e educazione informale. La conseguenza principale ha riguardato i nostri siti relativi ai paesi in rapida crescita economica come il Brasile, la Cina e l'India, dove le persone spesso auspicavano che i social media e altre tecnologie potessero ridurre ciò che veniva percepito come inadeguata erogazione di educazione formale in certe aree. Tuttavia, la percezione del valore educativo di questi servizi si connetteva a un più ampio desiderio di conseguire il successo. Questa più ampia prospettiva 
sull'impatto dei social media sull'istruzione emerge anche dal contesto etnografico, che rafforza a sua volta la lezione principale tratta dalla rassegna inziale della letteratura di riferimento: comprendere le relazioni tra apprendimento formale e apprendimento informale dipende dal riconoscimento del contesto sociale di entrambi. Mentre le prime tecnologie digitali, come i motori di ricerca, possono aver avuto un impatto più diretto sull'apprendimento autonomo, con i social media il fattore chiave è probabilmente il modo in cui le nuove comunicazioni facilitano sottili cambiamenti nelle relazioni tra studenti, docenti e genitori, mettendo in luce le dimensioni sociali critiche riguardanti ogni forma di apprendimento.

\section{Relazioni tra gli studenti: intimità, drammi, bullismo}

In tutti i nostri siti abbiamo osservato una tendenza comune riguardante il modo in cui gli studenti usano i social media per gestire le loro reciproche relazioni: i social media consentono a queste relazioni di diventare sempre più costanti, continue e personali. In molti casi gli studenti hanno apprezzato la più grande intimità alimentata da queste piattaforme; tuttavia, questo contatto ravvicinato e persistente è causa anche di occasioni di insulto e bullismo. Spesso l'intimità e l'intimidazione sono considerate due opposte esperienze, anche se i diversi siti provano che entrambe riflettono relazioni particolarmente forti alimentate dai social media.

Ci sono molti esempi di amicizie divenute più strette per l'uso dei social media tra gli studenti. Nel nostro sito della Cina rurale i social media erano considerati capaci di estendere la socialità del gruppo classe oltre il confine fisico della scuola. Nonostante gli studenti si incontrassero a scuola per gran parte del giorno, il gruppo classe trascorreva sempre più tempo online, lontano dalle aree scolastiche e fuori dall'orario normale. L'uso di 'QQ Groups' per organizzare regolarmente riunioni tra ex compagni di scuola ha facilitato ulteriormente la tradizione cinese di mantenere il gruppo classe come un'unità sociale anche in seguito.

Sia nel sito della Cina rurale che in quello della Cina industriale i social media rappresentavano generalmente uno spazio libero dalla sorveglianza dei genitori (contrariamente ad altri siti, di cui discuteremo dopo). Venivano considerati come uno spazio di più facile discussione tra pari, soprattutto su argomenti intimi o imbarazzanti. Nel sito della Cina rurale, circa la metà 
degli studenti medi intervistati ha risposto che i messaggi istantanei sarebbero il mezzo più adeguato per dichiarare a qualcuno il proprio affetto. Gli studenti universitari stavano usando sempre di più i social media per trovare partner nel campus.

L'intimità nella comunicazione sui social media non è un dato predeterminato, piuttosto essa è applicata in modo selettivo e intenzionale. Nel sito inglese, per esempio, il range delle piattaforme di social media corrispondeva a diversi gradi e livelli di intimità all'interno dell'amicizia tra studenti di scuole superiori. Come abbiamo rilevato nel Capitolo 1, Snapchat era considerata la piattaforma più intima, con studenti che mantengono tra i 10 e i 20 contatti con cui condividono regolarmente immagini. Inviare autoritratti brutti è anche segno di fiducia che questi non saranno inviati fuori dal gruppo $^{16}$. Twitter, invece, veniva utilizzato soprattutto per la comunicazione dell'intera classe.

Questo tipo di intensità di comunicazione sui social media potrebbe essere usato anche con finalità negative. Anche se derisioni e sfottò sono precedenti ai social media e si tratta di fenomeni ben studiati ${ }^{17}$, i social media consentono che questo tipo di comportamenti assumano forme diverse e anche che si manifestino, sempre di più, al di là dei cancelli di scuola. In Brasile, per esempio, i partecipanti spiegavano che i teen ager si sfidavano reciprocamente in azioni audaci, per esempio sottomettendo immagini indecenti di loro stessi sui gruppi di WhatsApp. Questo contenuto poteva eventualmente essere messo in circolazione da uno dei membri del gruppo come una forma di "vendetta porno". Anche se si tratta di un uso particolarmente vendicativo, esso implica una condivisione iniziale come segno di fiducia più in comune con l'esempio inglese di Snapchat.

Altri cambiamenti direttamente riconducibili ai social media nello studio sugli studenti della scuola inglese includevano l'estensione del dileggio dall'orario scolastico alla fascia 24/7; in questo modo gli studenti si sentivano più inclini a indulgere in questo tipo di derisioni nascosti dietro uno schermo piuttosto che faccia a faccia, aumentando l'utilizzo di "messaggi indiretti” come gli aggiornamenti di stato che non si rivolgono esplicitamente al destinatario o alludono vagamente al contesto e lasciando incerto colui al quale è riferito. Questi post indiretti spesso suscitavano conflitti e discussioni particolarmente stressanti, soprattutto per le ragazze.

Sinanan mostra come nel suo sito a Trinidad la crescente visibilità dovuta ai social media ha un impatto diretto su questo tipo di conflitti scolastici. 
Condividere video autoprodotti di zuffe tra studenti nel terreno di scuola ha creato una nuova forma di "evidenza" che viene intesa come particolarmente vera, nonostante sia soggetta a interpretazione deformata o a manipolazione. Alcuni postano queste zuffe nella speranza che il loro video diventi "virale".

Tutti questi esempi mostrano come i social media facilitino sia una più grande intimità sia una maggiore frizione tra gli stessi allievi della scuola, ma queste relazioni non sono mai del tutto indipendenti da connessioni associate ai genitori e ai docenti su cui ora ci soffermeremo. In Inghilterra, infatti, certi docenti ritengono che i problemi maggiori con i social media non risiedano tanto nel comportamento online inappropriato da parte degli studenti quanto in quello dei loro genitori. Dal momento che i social media consentono ai genitori di vedere direttamente, online, le liti tra i figli, essi desiderano ovviamente sostenerli, dando luogo spesso a situazioni in cui proprio i genitori (piuttosto che i figli) determinano liti ai cancelli della scuola.

E sono ancora i genitori a insistere che è la scuola ad essere responsabile di questi problemi e che dovrebbe essa stessa risolverli. In generale, gran parte di questo tipo di uso dei social media, e la tendenza delle giovani generazioni a dare corso alle loro relazioni sociali online, appaiono alieni a molti docenti e anche a molti genitori, alimentando spesso preoccupazioni importanti riguardo al fatto che i social media indicano questo tipo di passione tra i giovani. In risposta vediamo anche una significativa riconfigurazione delle relazioni docente-studente e genitore-figlio dal momento che questi gruppi cercano di appropriarsi dei social media per ridefinire e rimodellare a loro volta le loro relazioni con i giovani.

\section{Relazioni docente-studente: tra sorveglianza e impegno}

In molti dei siti una credenza costante riguardante il fatto che l'uso dei social media da parte dei giovani ostacolerebbe i loro risultati nel processo educativo, crea una particolare ambivalenza tra i docenti. Il sito in crescita "New Literacy Studies" (NLS) è utile per far luce sulla tendenza degli approcci teorici e politici a costruire un grande divario tra la scuola e ciò che è fuori da essa, spesso relegando tutte le attività piacevoli ai contesti non scolastici, ma anche assumendole come frivole e non incoraggianti l'apprendimento ${ }^{18}$. I docenti spesso sono chiamati ad avere il compito di monitorare questi 
confini vietando social media e ICT all'interno della scuola. Tuttavia, essi sono spesso molto consapevoli dell'importanza dei social media nella comunicazione tra i giovani e ne percepiscono pertanto il loro ruolo potenziale nell'educazione e nello sviluppo delle giovani generazioni. Pur nondimeno, il nostro sito italiano era del tutto contrario a questa generalizzazione e quello brasiliano lo era parzialmente.

Diversi studi basati su NLS ${ }^{19}$ hanno sostenuto la necessità di approcci didattici che incorporino nei curricula scolastici le abilità nell'uso tecnologico sviluppate dagli studenti a casa ${ }^{20}$. Così facendo, essi sfidano il presupposto non solo che la scuola sia il giudice più qualificato riguardo a quali tecnologie dovrebbero essere insegnate, ma anche che il sapere insegnato a scuola sia utile per la vita mentre quello acquisito a casa sia incidentale. Questo capitolo non intende riproporre questi importanti dibattiti pedagogici. Piuttosto, in linea con il nostro focus sulla socialità, si concentra invece sui rapporti mutevoli tra studenti e docenti che i social stanno aiutando a rendere più facili.

Molti docenti sono consapevoli anche del ruolo che i social media possono svolgere nel ridefinire le relazioni tra docente e studente. Una questione chiave per molti docenti sembra riguardare se sia appropriato essere amici dei loro studenti e, se lo è, come stabilire e mantenere confini e distanze adeguati tra loro e gli studenti attraverso i profili dei social media.

L'esempio più chiaro lo fornisce il sito dell'India del sud. Qui esiste un grado di ambivalenza e incertezza particolarmente alto riguardante il modo migliore di conformare la possibilità di stringere amicizia abbastanza egualitaria che i social media facilitano con il sistema fortemente gerarchico tipicamente avvertito nella relazione docente-studente. Venkatraman nota come in questo contesto i docenti spesso si trovino presi tra il loro ruolo di protettori nella cura degli studenti (che necessita di un impegno nel loro monitoraggio) e il divieto ufficiale nell'uso dei social media (sia per gli studenti sia per i docenti) all'interno di molte scuole.

Una delle ripercussioni di questa apparente contraddizione riguarda il modo in cui l'adeguatezza di una relazione on line docenti-studenti sia influenzata dall'età e dal genere degli studenti. I più entusiasti a stringere amicizia con i loro docenti sono generalmente gli studenti medi (dagli 11 ai 15 anni). Venkatraman descrive come essi si sentano orgogliosi di fare amicizia con i loro docenti su Facebook e quanto ci tengano a far sapere ai loro amici di questa nuova relazione online. Venkatraman attribuisce ciò 
al fatto che gli studenti medi riconoscono ai loro docenti un alto grado di autorità, ragione per cui non vedono l'ora di esservi associati. Egualmente, molti docenti desideravano fare amicizia con i loro studenti perché questo consentiva loro di monitorarne le attività online, realizzando il dovere di cura nei loro confronti.

Di contro, Venkatraman nota che proprio a causa di questo monitoraggio, gli studenti più grandi (dai 16 ai 18 anni) erano meno entusiasti di stringere amicizia con i loro docenti sui social media. Molti studenti (soprattutto maschi) hanno espresso la preoccupazione che questa amicizia avrebbe violato la loro privacy. Di questo avviso erano anche i docenti, essi stessi riluttanti a stringere amicizia con gli studenti più grandi, per il fatto che questi ultimi avrebbero potuto osservare aspetti della loro vita personale attraverso i social media. Venkatraman sostiene che in questi casi a far decidere se si sarebbe stretta amicizia con i docenti era il grado di prossimità e fiducia già esistente nelle loro relazioni offline.

Il sito del sud Italia verosimilmente aveva barriere persino maggiori rispetto all'amicizia online tra studenti e docenti. Qui i docenti si mostravano riluttanti a usare i social media e descrivevano se stessi come antiquati e riluttanti a impegnarsi su queste piattaforme. Temevano anche che i social avrebbero potuto indebolire quello che, secondo loro, era il 'classico' modello educativo secondo cui la conoscenza viene impartita da docenti istruiti a discenti inconsapevoli.

Leccezione più significativa a questa tendenza dei docenti a essere riluttanti nello stringere amicizia con gli allievi si trova in Cina. Ciò è probabilmente dovuto sia alla specificità delle piattaforme nazionali sia alle norme che regolano cosa è possibile postare e condividere online. Innanzitutto, le piattaforme più diffuse come QQ e WeChat non consentono di postare sulle pagine altrui e limitano o non permettono di taggare foto. Questo rafforza il controllo dell'utente su quanto compare sul proprio profilo e limita le opportunità di postare contenuti potenzialmente imbarazzanti sui profili personali dei social media. Pertanto, l'aspetto più rilevante dei social, la condivisione di post tra piccoli gruppi di amici visibili all'esterno, in Cina è molto meno controverso rispetto a molti altri siti. Di conseguenza pone ben pochi problemi riguardo al fatto che studenti e docenti vedano i rispettivi post. Anche se in Cina i docenti apparivano meno ansiosi in riferimento all'amicizia con gli studenti, rispetto agli altri nostri siti, esiste ancora una barriera significativa dal momento che alcuni docenti non ritengono di 
dover andare online. Gli studenti apprezzavano quei docenti che usavano i social media e talvolta trovavano più facile chiedere loro di aiutarli nei compiti.

Anche a Trinidad si riteneva che l'uso dei social media aiutasse a dominare l'ansia nella relazione studenti-docenti. Sinanan ha rilevato che all'interno della scuola le persone tendono a usare un inglese più formale mentre quando comunicano sui social media gli studenti si trovano più a loro agio nell'uso del dialetto locale. Di conseguenza, alcuni studenti con minor fiducia in se stessi, riluttanti a parlare in inglese formale in aula, preferiscono porre domande ai loro docenti sui social, fuori dalla scuola e in dialetto. In questo modo, riescono a impegnarsi maggiormente sia nei confronti degli insegnanti sia rispetto al lavoro scolastico.

Gli esempi di questa sezione hanno mostrato che in tutti i nostri siti i social media hanno ridefinito le relazioni docenti-studenti. Fornendo legami continui più forti fuori dallo spazio scolastico, sono diventati possibili nuovi modi di visibilità e di monitoraggio creando interessi sia per gli studenti che per i docenti. Di contro, questi nuovi social hanno determinato nuove strade di comunicazione, nuove modalità di impegno e, in alcuni casi, relazioni più egualitarie rispetto al passato.

\section{Relazione genitori-figli: la mediazione nello studio}

Il terzo lato del triangolo - diventato sempre più centrale negli studi sui social media negli Usa e in Europa grazie ai lavori di Livingstone, boyd e Clark, tra gli altri, - riguarda la relazione dei genitori sia con la scuola sia con i loro figli. Qui il tema distintivo è l'obbligo morale di allevare la prole in un certo modo e come questa loro idea di educazione possa differire da quella immaginata dalla scuola e dagli insegnanti e il ruolo dei social media nel mediare questo tipo di scarti.

Il caso più significativo (e probabilmente più apprezzato) di interazione docenti-scuola attraverso i social media è fornito dal nostro sito inglese. Qui una scuola primaria (con allievi di età compresa tra 5 e 11 anni) ha avviato un blog e un account di Twitter e posta immagini del lavoro che gli studenti svolgono ogni giorno. Il blog ha riscosso un successo immediato tra i genitori. Ha rappresentato una soluzione a un problema annoso, riguardante la difficoltà di molti genitori di avere resoconti dettagliati su quanto i loro 
bambini hanno fatto a scuola, spesso causando preoccupazioni circa l'efficacia della strutturazione del tempo scolastico da parte della scuola. In questo caso, postare le prove degli studenti (per esempio, disegni o poesie scritte) mitiga i timori dei genitori consentendo loro di rielaborare l'azione di mandare i figli a scuola come la realizzazione di un dovere genitoriale piuttosto che una sua inadempienza, facendo crescere la relazione genitori-insegnanti incommensurabilmente ${ }^{21}$.

Nel sito del nord del Cile, similmente, le scuole postano foto di eventi interni alla scuola, come seminari e attività speciali. Haynes rileva una sensazione tra i genitori riguardante il fatto che in città, man mano che i figli crescono, l'istruzione - a parte l'apprendimento di abilità pratiche - diventa sempre meno importante; si ritiene che la gran parte delle donne diventino maestre nelle scuole materne o lavorino nei supermercati locali mentre gli uomini lavorino nelle miniere o al porto. L'impegno su Facebook da parte delle scuole locali è uno sforzo per coinvolgere i genitori in modo che si riaccenda il loro interesse per l'educazione dei figli.

Un ultimo esempio di scuola attiva sui social media per gestire le relazioni con i genitori viene dal sito del sud dell'India. In questo esempio una scuola di più alto stato stava reagendo al danno che riteneva che l'uso dei social stesse causando alla sua reputazione. In questo caso il problema erano i genitori che consideravano che il loro dovere nei confronti dei figli si estendesse sino a riguardare lo scambio con la scuola. Aprirono un gruppo su Facebook per discutere potenziali cambiamenti nella trasmissione dell'istruzione e nei compiti dei loro figli e per fornire un più generale supporto. Tuttavia, ben presto il tono delle conversazioni divenne sempre più acceso, scivolando nel gossip e segnalando aspetti critici di diversi specifici professori della scuola.

Questi gruppi su Facebook attirarono l'attenzione del preside che in risposta cercò di esercitare un controllo su di loro. Incoraggiò alcuni genitori a formare un distinto gruppo su Facebook gestito da genitori che facevano parte dell'Associazione Genitori-Docenti ${ }^{22}$ della scuola, con rappresentanti della scuola che esercitavano un ulteriore controllo sul gruppo. Venkatraman rileva che un genitore riteneva che questo nuovo gruppo, strettamente connesso con l'attiva partecipazione della scuola, portasse un maggiore senso di comunità alle loro attività online. Per la scuola, l'apertura di un canale di comunicazione sui social media fu vantaggiosa. Tuttavia essi continuarono a scoraggiare la comunicazione docenti-genitori attraverso piattaforme tipo 
WhatsApp per il timore che la condivisione di un numero di cellulare personale di un docente con i genitori avrebbe sottoposto i docenti a continue domande da parte loro. L'esempio del sud dell'India dà il senso dello sforzo che le scuole fanno nell'uso dei social media per incanalare il senso del dovere dei genitori nei confronti dei figli e dell'investimento nella loro istruzione in un modo che queste organizzazioni ritengono conducente.

Di converso, nel sito del sud Italia la comunicazione attraverso i social media tra scuola e genitori appare piuttosto limitata sino al punto che i genitori spesso finiscono con il discutere tra loro le questioni sull'istruzione. Questo era collegato al ruolo esasperato che i genitori avevano nell'organizzare l'istruzione dei loro figli: aiutarli nei compiti a casa e impegnarsi per l'organizzazione di attività extracurricolari qui è molto comune. Nicolescu nota che ciò viene considerato come un aspetto del dovere morale della madre nei confronti del proprio figlio. Così, Facebook diventava un luogo particolarmente importante nel quale si condividevano con altre madri le acquisizioni dei propri figli che venivano commentate tra amiche.

Nicolescu rileva che queste pratiche portavano anche i genitori a volere che i loro figli usassero i cellulari sin dai dieci anni circa e i social media sin dai tredici anni. Questo è in contrasto con il divieto da parte delle scuole riguardo ICT e social media. I genitori tuttavia ritenevano che l'uso dei social media sarebbe stato d'aiuto perché soddisfaceva il desiderio che i loro figli avrebbero affrontato la vita in modo analogo ai loro pari, in termini di accesso non solo a specifiche tecnologie ma anche a certe opportunità che queste tecnologie avrebbero potuto fornire loro.

L'aspetto negativo di ciò, come si nota in alcuni dei siti, riguarda il fatto che i social media vengono usati sempre di più dai genitori per valutare la loro competenza nella gestione dei figli, e portando i genitori ad essere competitivi in riferimento al modo in cui i figli si confrontano con i loro pari. In Inghilterra, i genitori avevano in passato l'abitudine di confrontare i progressi dei figli all'interno di gruppi familiari che comprendevano bambini di età diverse. Lo sviluppo dei corsi pre-natali e dei gruppi di interesse per neonati e bambini piccoli, ha creato una situazione nuova in cui i confronti fra bambini che hanno esattamente la stessa età comincia dalla nascita, $o$ addirittura prima e continua nell'ambito del sistema educativo. Questo è a sua volta correlato a una struttura classista fortemente radicata nella società inglese, che si esprime sempre di più attraverso il tipo di scuola alla quale un bambino riesce ad avere accesso (o per le famiglie più ricche dal tipo di scuo- 
la privata che possono permettersi). Varianti di questo approccio imperniato sulla differenza fra scuole migliori e peggiori esistono anche in Cina e a Trinidad, sebbene in questi casi giochino un ruolo maggiore gli esami fortemente selettivi necessari per accedere alle scuole migliori. In ogni caso, tutto ciò finisce con l'esacerbare la tendenza alla competizione fra genitori, che oggi viene rafforzata annunciando i successi dei propri figli su Facebook e monitorando costantemente i progressi degli altri.

In questo paragrafo abbiamo visto come i social media possano ridurre le discrepanze fra diverse visioni di quello che dovrebbe essere il percorso educativo di un bambino. In alcuni casi, le scuole nei nostri siti in Inghilterra, nel Cile settentrionale e nell'India meridionale hanno provato ad affrontare il problema interagendo con i genitori attraverso i social media. In Italia, e in un caso isolato del nostro sito inglese, queste diverse visioni del dovere dei genitori possono portare a un punto morto o anche accrescere la competizione fra i genitori stessi.

\section{Conclusioni}

Per quanto riguarda l'impatto sul sistema educativo e sui giovani, il nostro studio ha potuto avvalersi di una letteratura di riferimento che si è sviluppata rapidamente a seguito dell'interesse dei governi e dell'opinione pubblica di stabilire se i social media stessero distruggendo o facilitando la formazione degli studenti in paesi come gli Stati Uniti o l'Europa. Le ricerche di Livingston sono state spesso finanziate dal governo, mentre quelle di Ito dalla fondazione MacArthur che è fortemente impegnata a sostenere gli studi pedagogici. Di conseguenza, si tratta di un ambito con un'attività di ricerca consolidata. Come emergeva dai loro lavori, come da quelli di boyd e Clark, cominciava ad essere chiaro che i social media, per la loro stessa natura, accendevano i riflettori sul contesto più ampio dell'apprendimento - in particolare sul ruolo dei genitori, ma anche su quello della classe sociale di appartenenza. Questo fornisce una chiave d'accesso ideale ai risultati della nostra ricerca etnografica. In ciascun singolo caso abbiamo riscontrato che proprio queste relazioni più ampie fra gli studenti stessi, fra insegnanti e studenti e fra insegnanti e genitori, svolgono un ruolo centrale nel comprendere perché i social media vengano percepiti a volte come uno strumento per ridurre una certa inadeguatezza nell'erogazione formale dell'istruzione, 


\section{Educazione e giovani}

offrendo rotte alternative e più informali verso l'acquisizione di nuove conoscenze, a volte come una distrazione dall'apprendimento, o persino come una causa diretta di interazioni negative fra gli alunni. 



\section{Capitolo 6 \\ Lavoro e commercio}

Dato che così tanti individui e aziende hanno un interesse legale nel guadagnare dal valore futuro delle azioni delle aziende di social media, non sorprende che il giornalismo focalizzato sull'uso e il potenziale dei social media nel commercio sia spesso letto, commentato e condiviso in modo entusiasta. C'è però anche un chiaro interesse popolare per questioni del tipo se i social media agevolino la comunicazione durante il lavoro o se distraggano gli impiegati, o se sia valido per le aziende investire più tempo e denaro nel marketing sui social media, e anche quanto i social media estendano la capacità del commercio di manipolare e attrarre clienti.

La ricerca di Venkatraman sul sito del'India del sud si concentra specificamente sull'impatto che i social media hanno sulle relazioni fra lavoro e non lavoro. La seguiamo insieme ad altri quattro punti. La nostra seconda parte esamina il modo in cui gli individui usano i social per trovare lavoro. $\mathrm{La}$ terza considera le aziende di social media stesse e l'impatto della pubblicità fatta sui social media, una strategia in crescita per ottenere fondi. La quarta parte riguarda il potenziale dei social media per lo sviluppo di e-commerce, scambio e piccole attività imprenditoriali. La parte finale contribuisce a un'analisi antropologica delle categorie di lavoro e commercio, e esplora le diverse idee che le persone hanno sul denaro e le sue relazioni con la famiglia e altri valori.

\section{La relazione tra lavoro e non-lavoro}

Una delle conseguenze più notevoli e significative di internet ha riguardato una radicale trasformazione della relazione fra posto di lavoro e casa. Sin dall'inizio della Rivoluzione industriale in Inghilterra coloro che detenevano il controllo del lavoro hanno cercato di imporre una rigida dicotomia, tentando di isolare completamente il posto di lavoro da ambiti e contatti familiari e personali $i^{1}$. Un importante breccia in questo muro fu provocata dall'email e poi dal telefono cellulare ${ }^{2}$; più recentemente social media e smartphome hanno in molti casi lasciato in rovina questa separazione, 
benché il grado di distruzione varii a seconda della regione e del tipo di attività. Tendiamo a vedere le innovazioni commerciali come allineate in modo naturale agli interessi affaristici che le producono, quindi è importante riconoscere casi come questi dove la tecnologia ha drasticamente ridimensionato un principio capitalista precedentemente santificato, la separazione fra lavoro e non-lavoro.

Questo è particolarmente importante da una prospettiva antropologica, dato che la disciplina è stata sempre impegnata a interpretare il lavoro dentro il più ampio contesto della vita delle persone, e per questo si è anche opposta a una dicotomia così rigida fra lavoro / non-lavoro 3 . Per di più molti antropologi studiano parti del mondo dove tali regimi di lavoro non sono pienamente stabiliti. In India, per esempio, lavori come la tessitura sono tradizionalmente delegati alle famiglie che lavorano da casa, mentre il sistema industriale ha una presenza molto più diradata che in Europa. Anche nelle industrie, gli operai in India hanno continuato a cercare di sfruttare i legami di parentela nel reclutamento e a rispettare i legami tradizionali con la casta ${ }^{4}$. In alcuni settori tuttavia le pratiche occidentali di separazione fra famiglia e posto di lavoro sono state occasionalmente stabilites.

Come in molte altre zone, i social media hanno spezzato queste barriere e agevolato la comunicazione non di lavoro sul posto di lavoro relativamente a storie d'amore, passatempi e socializzazione. Si usavano sistemi di chat di Office o di WhatsApp fra impiegati, i loro amici e partner per trasmettere rassicurazioni amorose, chattare su ciclismo, cricket e nuovi film, e per organizzare compiti familiari essenziali. Un marito può pianificare con la moglie impiegata in un'altra azienda chi prenderà la figlia da scuola. Una giovane lavoratrice madre invia un messaggio vocale con WhatsApp al telefono di sua madre per giocare con il suo bambino a casa.

In molti dei nostri campi di ricerca c'erano discussioni riguardo al fatto se i social media rappresentino una distrazione dal lavoro o qualcosa di utile. Nel campo di ricerca italiano c'era il pragmatico riconoscimento che certi lavori sono semplicemente noiosi. Per un assistente alle vendite senza clienti i social media sembravano un ragionevole antidoto alla noia, con il risultato che in questo modo era meno probabile che lasciasse il lavoro. Lavoratori come gli informatori brasiliani di Spyer trovavano troppo semplicistica la domanda se i social media fossero buoni o cattivi per il lavoro: erano entrambe le cose. Può essere una soluzione efficiente condividere informazioni sulle opportunità di lavoro e può essere anche utile per combattere la noia 
- come quella dei vigilanti che lavorano di notte - ma ha ugualmente un effetto negativo sul lavoro in termini di attenzione e tempo.

Una simile ambivalenza nei posti di lavoro è stata notata nel campo di ricerca inglese. I lavoratori in una ditta di medie dimensioni percepivano che questa era diventata troppo grande per incontri regolari faccia a faccia; non la sentivano più come una "famiglia". Usarono Facebook per resuscitare parte della loro precedente vicinanza; ciò conferì loro un accesso senza precedenti alle vite private dei colleghi. Il risultato è che ora gli argomenti di calcio e televisione possono essere integrati con conversazioni sulla famiglia e la vita domestica. Benché questo consenta un grado maggiore di familiarità tra i colleghi può anche diventare intrusivo: per esempio, quando un capo aveva accesso a più informazioni personali di quanto i lavoratori gradissero, o quando gli impiegati si trovavano a disagio per via delle opinioni politiche di un collega espresse sui social media.

Il nostro esempio più significativo di reintegrazione di lavoro e vita familiare deriva dal campo di ricerca in Cile. La principale industria della città è una miniera di rame, che prevalentemente impiega uomini; questi lavorano periodi di settimane, e vivono in dormitori sul posto. Durante questo periodo usano i social media per mantenere la comunicazione con la famiglia, per tenersi dentro la vita familiare; le conversazioni spaziano dall'organizzazione della festa di compleanno dei figli al pagamento della bolletta della luce.

I social media hanno anche un impatto forte sulle relazioni delle donne con le famiglie di cui esse sono diventate parte rilevante della forza lavoro, con riferimento al tema della cura dei figli. In Inghilterra le donne oggi formano il 47 per cento della forza lavoro, ma nel sito inglese di solito prendono un'estensione del congedo per maternità, spesso un periodo di intenso uso di social media.

Il nostro studio comparativo ha incluso anche la questione se i social media siano consentiti nei luoghi di lavoro (Fig. 4.21), ma le risposte sono state molto specifiche, rendendo difficile la generalizzazione ${ }^{6}$. Per esempio, in Brasile l'uso dei social non è permesso dai direttori ai lavoratori degli hotel - parte maggioritaria della popolazione del villaggio. Nondimeno, come nella maggior parte di siti, anche se l'uso dei social media ufficialmente non è permesso molte persone trovano modi per aggirare questo regolamento. 


\section{Trovare e ottenere lavoro}

L'unica più ovvia connessione tra social media e l'ottenimento di un lavoro è la piattaforma LinkedIn. Rivendicando 300 milioni di utenti ${ }^{7}$, questa piattaforma è prevalentemente usata nel commercio e specialmente per il reclutamento professionale. Nel nostro sito inglese non era mai menzionata al di fuori del settore commerciale, ma per alcuni che lavoravano nel commercio era di gran lunga la piattaforma social più importante. Una persona controllava sempre se i candidati a posizioni lavorative stavano su LinkedIn come un criterio chiave per decidere chi assumere. Le persone che lavoravano in imprese locali di piccole dimensioni mostravano un ethos fortemente collaborativo piuttosto che competitivo, spesso incontrandosi faccia a faccia ma anche ben consapevoli del ruolo di LinkedIn. Dopo gli USA, la nazione con la più ampia base di utenti LinkedIn è l'India, seguita dal Brasile. Era, tuttavia, molto poco probabile imbattersi nel genere di lavoratori a basso reddito e con scarso livello di istruzione studiati da Spyer, benché sia certamente nota agli indiani del settore IT.

LinkedIn è dunque importante in alcuni siti (maggiormente quello inglese), ma di fatto le persone si appropriano creativamente di altre forme di social media al fine di accrescere le opportunità di lavoro. Il sito di ricerca in Italia meridionale di Nicolescu è stato una rivelazione nel mostrare come i social media possono aiutare le persone a trovare impiego. I locali sentivano che l'istruzione formale aveva valore limitato per trovare lavoro. Tradizionalmente le persone tendevano a trovare impiego mediante i network familiari e sociali, in parte perché questa regione è celebre per il lavoro più artigianale, come il cibo di alta qualità e la sartoria di lusso. Tali attività oggi sono però in calo e nuove forme di impiego sono meno sensibili all'influenza di questo tipo di reti familiari.

Come spesso accade, i social media come Facebook favoriscono modi di espressione visivi piuttosto che solo testuali (vedi il Capitolo 11). Su Facebook le persone imparano a creare post di stile e intelligenti, echeggiando il ruolo che lo stile ha in lavori tradizionali più artigianali. Con più di 200 organizzazioni culturali diverse, 437 imprese artigiane ${ }^{8}$ e 116 bar e ristoranti presenti nel campo di ricerca in Italia del sud, ci sono molte opportunità per la promozione visiva come la grafica, in parallelo a un'espansione dei settori commerciali collegati, inclusa la pubblicità e le pubbliche relazioni. Senza volerlo, quindi, Facebook è diventato un terreno trainante e uno spazio di esibizione per abilità che stavano diventando una strada sempre più impor- 
tante da seguire. Questo si adatta a persone con elevato capitale culturale o educativo dato che, anche se poco pagate, si tratta di occupazioni di un certo prestigio che mettono in risalto le loro capacità artistiche. Come mostra Nicolescu, tutto ciò non serve alle persone con scarso capitale culturale e d'istruzione, ma le persone con il background giusto possono capitalizzare sui social media in questo passaggio da più antiche abilità artigianali a questo mondo nuovo, più internazionale, di design online.

In altre aree la chiave all'impiego resta l'istruzione. Come indicato nei capitoli precedenti, Venkatraman ha trovato una distinzione netta tra le scuole più privilegiate, che tendevano a proibire l'uso dei social media, e le scuole per studenti a basso reddito. Queste ultime incoraggiano l'uso dei social media, in parte nella speranza che prepari gli alunni poveri a impieghi nel settore IT. In questo sono diventate molto più permissive rispetto alla norma dei paesi più sviluppati', anche se non è chiaro fino a che punto l'uso dei social media alla fine aiuti a ottenere questo tipo di impiego. Più in generale, il settore indiano dell'IT ha cercato ultimamente di reclutare di più mediante un'apparente meritocrazia, concentrandosi su credenziali di formazione ${ }^{10} \mathrm{e}$ non su legami di casta o familiari.

Anche in questo settore modernizzato Venkatraman ha tuttavia trovato che WhatsApp giocava un ruolo significativo nel riportare i processi di reclutamento a strade più consuetudinarie; gli impiegati in servizio passavano informazioni sui posti liberi o sull'azienda ad amici qualificati e a membri della famiglia allargata, spesso prima che l'offerta di lavoro fosse ufficialmente resa pubblica.

Spyer nel titolo del suo libro usa il termine "classe emergente", dato che in Brasile l'occupazione è un aspetto dell'esperienza recente di aumento della mobilità sociale di più di metà della popolazione e dell'aspirazione a ottenere un livello decente di reddito e consumo e almeno un parziale capitale culturale e d'istruzione. Il termine 'emergente' si poteva ugualmente applicare ai nostri campi di ricerca in Cina e in India, due aree che rappresentano forse la maggioranza della popolazione mondiale oggi. In questo contesto l'impiego non è solo attività lavorativa e fonte di reddito: lega le persone a un mondo formale più ampio che comprende non solo attività bancaria, regolamentazione, orari e lo stato, ma spesso anche alfabetizzazione e mobilità. Per chi in Brasile programma di frequentare un college, i social media diventano il posto per condividere opportunità o consigli per riuscire nel colloquio di lavoro. I social media per questo diventano un modo della 
solidarietà per questa classe emergente. Si possono utilizzare anche per collegare opportunità di lavoro correlate. Analogamente al Brasile, un idraulico nel nostro sito inglese userà i social media per connettersi con carpentieri o imbianchini, quindi un'occasione per cui spesso tutti possono ottenere del lavoro grazie a uno di loro.

\section{La rilevanza delle aziende di social media}

Il nostro progetto non riguarda le aziende di social media stesse in quanto datrici di lavoro. Il numero di impiegati è piccolo, e ci saremmo molto sorpresi se avessimo incontrato un unico impiegato di questo tipo in ciascuno dei nostri campi di ricerca. Eravamo molto più interessati a misurare quanto gli utenti si preoccupano dell'influenza di queste aziende sulle loro vite: per esempio, se erano interessati all'uso potenziale dell'enorme quantità di informazioni personali cui queste aziende hanno oggi accesso.

Abbiamo trovato scarsa evidenza di tale preoccupazione. Quando Miller ha indicato che Facebook aveva perso la sua attrattiva per i giovani ${ }^{11}$, ha sostenuto che questo accadeva non perché si preoccupassero di che cosa Facebook avrebbe potuto fare con i dati che li riguardavano, ma per ciò che i loro genitori avrebbero potuto fare. Il fatto che Facebook come azienda detenesse sia Instagram sia WhatsApp non ha cambiato il discorso pubblico. Nei nostri siti le persone comparano, mettono in contrasto e usano diversamente Instagram, Facebook e WhatsApp come piattaforme alternative oppure complementari non curandosi affatto della loro proprietà unica, una questione di cui la maggior parte delle persone sembra in generale inconsapevole e alla quale si disinteressa ${ }^{12}$.

Nel determinare la rilevanza delle aziende, conviene tornare a riflettere sulle loro storie descritte nel Capitolo 2. Quanta differenza avrebbe fatto, per esempio, se Facebook non fosse mai stato inventato? In Cina comunque non si usa. Presso altre popolazioni numerose come in Brasile e in India è stato Orkut a spopolare tra i social media; il motivo del passaggio a Facebook ha riguardato prevalentemente l'emulazione delle aree metropolitane. Se le cose fossero andate diversamente e Orkut avesse annullato Facebook divenendo il principale giocatore mondiale, la maggior parte delle persone se ne sarebbero accorte a stento. Nello stesso tempo, la scomparsa di Orkut (diventato proprietà di Google) e il relativamente scarso successo 
di Google+ indicano anche i limiti del potere delle corporazioni. Persino compagnie enormi, cross-commerciali come $\mathrm{QR}^{13}$ possono fallire. Abbiamo prova che gli utenti possono preoccuparsi di aggiungere una componente video alla comunicazione, per esempio, ma sono molto meno interessati se la ottengono tramite Skype, FaceTime o Facebook, o se un messaggio è di WhattsApp, Facebook o semplicemente del loro cellulare. Data la polimedialità dell'ambiente, è probabile che a entrare in gioco tra queste opzioni sia un valore culturale, qualcosa di gran lunga più importante per i nostri informatori delle diverse regioni, delle proprietà tecniche di ciascuna piattaforma o di chi ne sia il proprietario. C'è chiaramente un interesse verso Apple contro Android o Microsoft. Tuttavia, è probabile che anche questo sia per scopi culturali, per esempio per mostrare il proprio cellulare nuovo, piuttosto che per l'interesse nei confronti delle aziende in sé.

Riguardo alla questione della sorveglianza o del potere delle aziende, il senso del controllo monopolistico è forse maggiore in Cina dato il dominio della compagnia Tencent (che possiede le due piattaforme più popolari, QQ e WeChat). In Cina questo controllo è anche associato a uno stato monopolistico che, benché separato da Tencent, nondimeno interferisce nell'uso dei social media da parte delle persone, soprattutto per prevenire l'accesso a Facebook e Twitter. Come indica Wang, gran parte dello sviluppo commerciale delle aziende di social media in Cina è arrivato come esito di un'iniziativa governativa nel 1999 per espandere il settore, considerando che la Cina era indietro di molti decenni rispetto ad altri paesi in termini di tecnologie della comunicazione. La promozione dell'ICT come una della quattro "modernizzazioni” fu un successo spettacolare. Nel 2003 la Cina sorpassò gli USA come il più grande mercato telefonico mondiale ${ }^{14}$ e nel 2008 il paese aveva il più alto numero di utenti di internet $\mathrm{al} \mathrm{mondo}^{15}$.

Inoltre, l'impatto può essere più pervasivo. Come mostra McDonald, gli utenti di social media nel suo campo di ricerca nella Cina rurale erano sempre più dipendenti dal Tencent News Centre, loro principale fonte giornaliera di news, che diffonde aggiornamenti per le app. McDonald non è riuscito a trovare un solo utente che avesse disattivato tale funzione. La sua analisi mostra inoltre la differenza fra le news dei social media comparate con i network statali di news. Nei primi domina il crimine, seguito da argomenti generici come relazioni sentimentali, matrimonio, sesso. Il crimine riguarda anche molte vicende di corruzione, quindi dove la censura non viene sfidata la critica ai politici corrotti resta significativa. Sui social media 
tuttavia questa critica tendeva a incentrarsi su discussioni intorno a personaggi di altre aree. All'opposto, le persone erano più desiderose di parlare di questioni locali in situazioni informali faccia a faccia. Fuori dalla Cina sino a ora non ci sono indicazioni parallele riguardanti il controllo di Facebook sul contenuto di news a questo livello, mentre Twitter è visto come una strada che porta alle news piuttosto che come un creatore di contenuto. Di nuovo, se ci volgiamo alla Turchia sudorientale, il tema principale per le persone riguarda lo stato e non le aziende, e la minaccia che lo stato limiti l'accesso a siti come Twitter.

Un altro modo in cui l'attività commerciale può ledere i diritti degli utenti è mediante la pubblicità sui social media. Nel suo studio dell'uso commerciale dei social media sia nella città industriale sia a Shanghai, Wang ha trovato che relativamente poche persone dichiaravano di aver acquistato oggetti direttamente per via della pubblicità, ma molte erano state influenzate nei loro acquisti dalle opinioni di altre persone che avevano conosciuto sui social media (Fig. 4.16). Si tratta soprattutto di un modo indiretto, per esempio il riferimento a un ristorante di cui si è apprezzato il cibo. Mentre precedenti forme di pubblicità possono aver avuto un minore impatto sui social media, piccole attività commerciali hanno trovato nuove forme di pubblicità, per esempio raccogliendo "mi piace" su WeChat che usano per accostarsi ai consumatori ${ }^{16}$.

L'argomento della pubblicità mette in luce la questione più importante del controllo da parte delle aziende di social media e di quanto le aziende sanno sugli individui. Questo era un argomento diffuso nel sito inglese, dove i dati indicano che potrebbe esserci un difetto in ciò che molte aziende auspicano sia un piano finanziario sostenibile. AI fini del commercio, c’è oggi un enorme interesse nell'uso crescente di pubblicità mirata, in gran parte perché le aziende di social media, come molte altre aziende digitali, hanno individualmente fallito nel trovare un modello di business alternativo. Il problema è che mentre in Inghilterra le persone possono non essere troppo preoccupate dall'idea astratta di controllo, e nemmeno dalle questioni politiche sollevate dalle rivelazioni di Wikileaks nel 2001 e di Edward Snowden nel 2013, questi timori si fanno molto reali quando si sperimenta personalmente di essere destinatari di pubblicità talvolta decisamente inappropriate che possono derivare solo da una fonte che possiede una buona dose di informazioni private su se stessi. Ne erano esempi pubblicità che mostravano che le aziende sapevano di persone malate di tumore o andate 
in pensione. Sembra quasi che le aziende ignorino l'evidenza che questo tipo di pubblicità mirate possano davvero infastidire le persone piuttosto che costruire una relazione più stretta con l'azienda. Anche se la pubblicità mirata talvolta può occasionalmente persuadere le persone ad acquistare certe merci, è del tutto possibile che gli effetti negativi di questo costante ricordare quanto l'azienda conosce di te possa essere più dannoso ${ }^{17}$. Certamente si è trattato di una lamentela spesso espressa dagli informatori.

\section{Impresa e networking}

In diversi siti, social media e e-commerce più in generale si iniziano a vedere come uno strumento promettente per sviluppare business locali di piccole dimensioni. Nel nostro sito industriale cinese abbiamo incontrato A-mei, un'operaia. A-mei ha utilizzato le sue reti parentali e le immagini del sito di acquisti on line Taobao per fondare una vendita di cosmetici su WeChat, salvo poi scoprire che c'erano già troppe persone che facevano la stessa cosa, anche perché con i social media c'è davvero poco che ostacoli l'avvio di un commercio. Invece, una vendita di uova di galline allevate all'aperto e polli ruspanti nella stessa area ha avuto successo, ma in gran parte perché i clienti erano per lo più amici, parenti e colleghi. Questo tipo di imprese sono avviate per lo più da giovani donne in aggiunta al lavoro ufficiale e non come occupazione a tempo pieno. Più in generale McDonald e Wang sostengono che le raccomandazioni personali hanno di gran lunga più influenza su ciò che le persone comprano in Cina rispetto ai paesi occidentali, e questo alimenta la crescita di e-commerce su piattaforme come WeChat ${ }^{18}$.

McDonald nota che la mancanza di profili di vendita specifici sulle piattaforme di social media cinesi rende più difficile stabilire la presenza di un'attività, ma anche nella sua cittadina le persone usano i social media per promuovere imprese che si basano sulla clientela personale, come ristoranti o copisterie. Per esempio, si possono utilizzare il nome della azienda e la fotografia del negozio come foto identificativa del loro account utente. La chiave dell'utilizzo commerciale in Cina non è tuttavia l'uso diretto delle piattaforme di social media. Più importante è il modo in cui la piattaforma commerciale di Taobao ha sviluppato tratti sociali distinti da siti equivalenti non cinesi come Amazon. Questo è un caso di sviluppo ibrido, dove i siti web di e-commerce permettono alle caratteristiche dei social media di essere 
incorporate in essi. Su Taobao acquirenti e venditori hanno la possibilità di chattare direttamente. I prezzi possono essere fissati individualmente per specifici clienti, consentendo la negoziazione. Anche nella cittadina rurale di McDonald, almeno una donna gestiva il suo personale negozio Taobao vendendo vestiti online. In città è stato aperto anche un vero e proprio negozio Taobao che aiuta le persone nei loro acquisti Taobao online organizzando la consegna, altrimenti un ostacolo significativo all'e-commerce. Inoltre fornisce assistenza individuale, il che aiuta le persone a imparare a fare acquisti online, o chi non ha un conto bancario.

Analogamente c'è un più forte sviluppo in Cina dei servizi di pagamento online (spesso indicati come "portafogli digitali"), che si concretizzano in una monetizzazione più effettiva delle piattaforme di social. A volte ciò significa che i giovani spendono più direttamente sui social media, all'insaputa dei loro genitori. Tutto quanto detto sopra indica che piuttosto che cercare un effetto autonomo dei social media sul commercio, dovremmo considerare i social media come semplice parte di una nuova fusione di innovazioni personali, commerciali e comunicative.

In un modo leggermente diverso, la stessa conclusione si trae dal nostro campo di ricerca in Italia del sud. Anche qui gli imprenditori rappresentano il più esplicito esempio di uso dei social media. Sembrano stare perennemente al telefono. Possono avere due smartphone e aggiornano regolarmente i loro profili, personale e professionale, su Facebook. Eppure, di nuovo, non si tratta poi molto di uso diretto dei social media, dato che le limitate prove indicano che anche per attività come i parrucchieri la pubblicità su Facebook non è particolarmente efficace. Piuttosto questo massiccio uso da parte delle persone dedite al commercio ha più a che fare con l'espressività italiana e il desiderio di visibilità e di mettersi in mostra. Generalmente le persone qui sono a loro agio nel mescolare affari e uso personale con un senso comune di stile. Questo in parte perché gli affari sono stati tradizionalmente basati sulle relazioni personali, ma ancora di più perché la motivazione principale a monte del business non è il profitto, ma piuttosto il modo in cui esso mostra la posizione sociale e agevola la socializzazione, come fini in se stessi. $\grave{E}$ anche il modo in cui le persone rappresentano se stesse come buoni cittadini e ottengono prestigio sociale. Particolarmente attivi sono gli affari che cercano di coltivare lo stile e un senso di cool, come bar e ristoranti. $\mathrm{Al}$ contrario, gli usi più distanti e astratti dei social media come l'e-commerce sono di gran lunga molto meno sviluppati. 
La situazione a Mardin, nella Turchia sudorientale, ci aiuta a rifinire questo focus sulle connessioni personali. Costa sostiene che Facebook ha avuto un impatto considerevole sulle piccole attività, per esempio negozi, musicisti, agenti immobiliari, caffe, ristoranti e scuole private. In alcuni casi queste particolari forme di attività potrebbero non essere realizzabili altrimenti. Per esempio, un agenzia immobiliare usava Facebook per creare contatti con persone che non vivono a Mardin, inclusa la sua popolazione diasporica, e aiutava gli impiegati statali ovunque in Turchia a investire in proprietà immobiliari in città. Oltre a questa dimensione nazionale, i social media permettono a musicisti e artisti locali di entrare in contatto con persone in Europa, per richiedere e comprare strumenti musicali e equipaggiamento tecnico, o semplicemente per scambiarsi opinioni sul modo migliore di suonare una canzone. Il potere visivo di Instagram iniziava a divenire importante nella pubblicità della moda. Un ammonimento era però che i social media stavano rendendo l'età un fattore importante - soprattutto persone sotto i 30 guardavano queste opportunità, e le attività economiche svolte da persone più grandi raramente ne erano influenzate.

Una delle cose più rimarchevoli sul nostro sito cileno di Alto Hospicio è che, a parte pochi supermarket e un negozio di edilizia (di proprietà di Home Depot), ci sono solo pochi negozi, a conduzione familiare. La feria ('mercato') probabilmente vende di più di tutte queste attività formali messe insieme. È dove le persone comprano vestiti nuovi e usati, casalinghi, cibi pronti, cibi da cuocere, stoviglie per animali domestici, elettronica, ricambi per auto, attrezzi, dispositivi vari, ecc. Nondimeno, colpisce che uno dei nostri siti più grandi, con 100.000 persone, ha quasi la stessa quantità di attività commerciale. È difficile persino scovare un tabellone pubblicitario. In contrasto con la mancanza di business formale, dozzine di pagine sono fatte comparire all'improvviso su Facebook con nomi come "Buy and Sell Alto Hospicio". Questo fondamentalmente agisce come il sito USA Craigslist, dove le persone postano fotografie di qualsiasi cosa vogliano vendere (oggetti usati come vestiti, utensili, auto, case, case in affitto, strumenti, cibo pronto - il sushi è il più popolare, ecc.).

Altre persone avviano il loro business personale interamente tramite Facebook, vendendo cibo fatto in casa o vestiti e scarpe importati. Molte persone non hanno carta di credito e non si fidano della mail, per cui è molto raro che si ordinino oggetti online. Facebook ha dato però il via a un tipo di commercio online totalmente nuovo, interamente locale. Le persone 
dicono che si fidano dei social media più che di altre forme di e-commerce perché possono vedere chi sta vendendo loro qualcosa. Ciò si conforma alle più ampie osservazioni etnografiche su fiducia, legami personali e sospetto svolte in questo sito.

Via via che ci muoviamo attraverso i diversi campi di ricerca possiamo scorgere che in tutti i casi i social media creano nuove forme di imprenditoria. Il modo specifico in cui questo accade in ogni caso tende a dipendere da fattori locali, specialmente dal modo di vedere il lato personale come un fattore positivo o negativo delle transazioni commerciali. Per esempio, a Trinidad l'uso dei social media per usi commerciali è decisamente specifico. Un esempio è un negozio di fotografia la cui principale attività consiste nel fare servizi fotografici ai party dei trinidadiani con l'obiettivo poi di postare queste foto su Facebook; la presenza di questi fotografi è diventata uno dei modi in cui l'agenzia che promuove questi party ha attirato folle ${ }^{19}$. In più, un pubblicitario esperto di "social market" ha spiegato in un'intervista come le promozioni su Facebook tengano conto dell'orario della giornata, in relazione a quando le persone stanno mangiando, per esempio, o quando potrebbero recarsi in palestra ${ }^{20}$. Allo stesso modo, alcune attività come bar, parrucchieri o nightclub, dipendono da relazioni personali e, per questo, fanno uso dei social media. Per esempio, un bar a Trinidad che cercava di indirizzare il business verso una categoria di persone più esperte di media aggiornava regolarmente i suoi eventi e news.

Più in generale, tuttavia, Trinidad e l'Inghilterra si sono rivelati i due siti con evidenti limitazioni nell'uso commerciale dei social media in settori meno dipendenti da legami personali. Molti negozi e outlet commerciali nel nostro campo di ricerca inglese avevano tentato di usare i social media come Facebook, ma si poteva constatare come molti di questi siti online fossero abbandonati, molto semplicemente perché non valeva la pena dedicarvi il tempo che richiedevano. Ancora una volta, a meno di un forte elemento di personalizzazione, molti degli interessi commerciali locali colgono pochi benefici nell'usare i social media. Questo è vero anche a Trinidad, ma i motivi sono diametralmente opposti. Nel caso di Trinidad le persone preferiscono ancora la comunicazione faccia a faccia o il gossip come mezzi per ottenere informazioni sulla qualità di beni o servizi all'interno della città stessa. Nella loro tranquilla comunità di campagna conoscono da tutta la vita i proprietari dei negozi. Al contrario, nel campo di ricerca inglese questo può essere vero per quanto riguarda il macellaio o il fornaio, altrimenti i negozi e i 
servizi che vendono beni sono valutati in base ai prezzi e all'efficienza e non alle relazioni personali; spesso sono gestiti da gruppi etnici minoritari che sono tenuti a distanza. Qui è il desiderio di tenere separati denaro e relazioni personali che rende i social media inefficaci per le transazioni commerciali.

Infine, nel sud dell'India Venkatraman ha trovato che WhatsApp viene usato per modificare i network personali in modo da coordinare attività imprenditoriali. Poche giovani madri istruite (di 35 anni o meno), con alle spalle percorsi professionali ben pagati in azienda, volevano avviare attività imprenditoriali part-time dalle loro case. Queste spaziavano dalla preparazione di spuntini appena cucinati alla produzione di bigiotteria vivace e creativa o alla fornitura di supporto scolastico a domicilio per i bambini, e il target era rappresentato soprattutto da altre mamme con bambini. Tutti i loro annunci correvano mediante WhatsApp, preferito perché era economico, quasi simultaneo e facilmente accessibile dal cellulare.

In ogni caso, per imprenditori indipendenti orientati al business dei servizi che desiderano portare avanti un progetto più intenso, Facebook è la piattaforma più scelta, fornendo effettivamente un marketing a costo zero. Usha è una cantastorie professionista di 40 e passa anni. È stata chiara nel suo scopo di affermarsi come la cantastorie più richiesta, restituendo la cultura della narrazione orale dalle sue radici come una tradizione informale e rendendola disponibile nelle case a scopo formativo. In quanto imprenditrice autonoma, vede la tecnologia come la chiave sia per il marketing sia per l'organizzazione delle sue operazioni giornaliere. Di volta in volta ha usato Yahoo groups, poi Orkut e ora Facebook.

Usha ha individuato un potenziale per via della crescita delle famiglie nucleari, spesso prive di nonni. Per questo lei si offriva di insegnare ai genitori le abilità dei cantastorie. Vedeva anche che le aziende stavano mettendo in evidenza la necessità che anche i manager diventassero dei bravi narratori, quindi ha creato una pagina Facebook per se stessa, separando il suo profilo personale dalla sua pagina pubblica, collegata al suo lavoro. Postava storie, link a pagine sulla narrazione orale e narrazioni di storie da condividere tramite Facebook. Quindi ha postato fotografie delle sue sedute di narrazione, dove appare all'opera con diversi tipi di pubblico. Usha sentiva che le sue fotografie erano molto più d'impatto rispetto a qualsiasi testo. Soprattutto era contenta che Facebook le avesse fornito una piattaforma pubblicitaria a costo zero consentendole di mettersi sul mercato come imprenditrice indipendente.

Anche il campo di ricerca nel sud dell'India ha mostrato i temi particolari 
con cui si confrontano certi consumatori. Venkatraman ha scoperto che diversi uomini socialmente ed economicamente di caste basse usavano siti di e-commerce come Flipkart per acquistare T-shirt, scarpe, ciabatte e oggetti simili, dato che trovavano socialmente imbarazzante comprare in grandi magazzini di beni di marca. I commessi di questi negozi possono discriminare uomini di classi socialmente e economicamente basse. Senza carte di credito o di debito, facevano uso del sistema di pagamento in contanti alla consegna consentito da questo portale. Per di più vennero a conoscenza di Flipkart solo attraverso le sue pubblicità su Facebook, al quale avevano accesso tramite gli smartphone.

Questo aneddoto dall'India del sud ci ricorda che il commercio è una pratica enormemente diversificata; possiamo trovare storie che riflettono una gamma sorprendente di usi dei social media lungo tutti i nostri nove siti. E possibile però proporre qualche generalizzazione. In generale dalla nostra ricerca è derivata poca evidenza a sostegno di una forte spinta interna al commercio stesso per promuovere i social media come la chiave del marketing moderno. A parte LinkedIn, queste piattaforme erano in gran parte predisposte, e sono per lo più usate, per la comunicazione al di là del commercio, nell'ambito di piccoli gruppi e connessioni personali. Quindi, senza sorpresa, abbiamo trovato che i social media sono stati per lo più utili per quelle imprese commerciali che sono basate su legami personali e socialità di piccola scala. Per quanto riguarda i nostri informatori, l'impatto su operazioni commerciali di grande scala era molto più ridotto. Questa è l'area dove le persone si preoccupano per la diffusione di pubblicità mirata in quanto segno di indesiderate intrusioni nella vita privata ${ }^{21}$. Nello stesso tempo i più giovani in molte parti del mondo sono orami adusi a utilizzare gli outlet commerciali online come Amazon e Taobao, laddove quest'ultimo sta guadagnando le stesse caratteristiche di un social media.

\section{Valori più ampi}

Uno dei compiti specifici dell'antropologia è interrogarsi su ciò che a volte chiamiamo categorie "occidentali". Tendiamo a considerarle razionali, scientifiche e naturali mentre, comparativamente, quando leggiamo una descrizione di cinesi che bruciano soldi falsi ai funerali potremmo trovare strambo questo rapporto con il denaro. Eppure si tratta di qualcosa che potremmo 
spiegare facilmente mediante il concetto di cosmologie divergenti ${ }^{22}$.

Soggiacente a questa analisi della relazione dei social media e del commercio, c'è dunque l'esigenza di approfondire ciò che le persone concretamente intendono per cose come denaro, valore, scambio. Come esempio esamineremo innanzitutto il contrasto fra ideali cinesi antichi e tradizionali, e poi le convinzioni dei giovani contemporanei in posti come la Gran Bretagna e gli Stati Uniti. Un esempio da considerare è la monetizzazione di WeChat mediante il 'red envelope'. Questo si basa su un antica usanza consistente nel dare denaro in buste rosse durante festività come l'ultimo dell'anno e i matrimoni ${ }^{23}$. In questo caso però il denaro è digitalizzato e, in stile molto cinese, è introdotto un elemento di fortuna. 'Si racconta che dalla vigilia del Nuovo Anno cinese fino alle 4 del pomeriggio del primo giorno del nuovo anno cinese (31 gennaio 2014), più di 5 milioni di utenti hanno sperimentato la funzione di distribuire più di 75 milioni di buste rosse digitali' ${ }^{24}$, tutti utenti legati a WeChat. Mentre le buste rosse tradizionali andavano dalle generazioni dei più anziani alle generazioni dei più giovani, la versione WeChat è diventata più orientata da pari a pari.

Dobbiamo apprezzare il fatto che in Occidente l'ambito della vita privata e familiare è stato tradizionalmente definito in parte per opposizione all'area del denaro e della finanza ${ }^{25}$. Regalare denaro è percepito come meno personale che fare altri doni. In Cina il denaro e la finanza sono sempre stai visti come un aspetto integrante della vita familiare e intima, e non in opposizione ad essa. Il denaro in molte società asiatiche è considerato come il modo appropriato e forse il migliore di mostrare affetto e attenzione in famiglia, e di mostrare che i propri sentimenti sono autentici ${ }^{26}$. In alcuni casi i contanti possono essere visti come più appropriati per un regalo fra persone che sono intime rispetto a un regalo.

Inoltre, se guardiamo invece ai giovani in Gran Bretagna e in USA troviamo un parallelo interessante. I social media hanno facilitato un'intera gamma di pratiche come il Kickstarting, il Crowdsourcing e il Couch Surfing che, dobbiamo ammetterlo, erano quasi del tutto non contemplate nel nostro progetto di ricerca. Anche se alcune di queste sono legate alla possibilità di guadagno, per la maggior parte sono proprio l'opposto. Si basano sull'altruismo, incoraggiano le persone a dare denaro e prestazioni d'aiuto senza aspettarsi alcun ritorno immediato, e si collegano ad altre tendenze, come Open Access e Open Source, che sono importanti per l'ethos delle nuove pratiche digitali ${ }^{27}$. In tal senso non sono come la de-mercificazione della 
musica o dell'intrattenimento, dove piratare o ottenere qualcosa gratuitamente è chiaramente fatto nell'interesse delle persone coinvolte. In alcuni di questi altri nuovi movimenti, lo stretto interesse personale scompare in ciò che gli antropologi chiamano "scambio generalizzato o reciprocità generalizzata" ${ }^{28}$, dove si auspica che se siamo tutti generosi nel breve termine tutti ne beneficeranno nel lungo termine, inclusi noi stessi. In questi nuovi movimenti le persone investono in idee e sviluppi che potrebbero non essere finanziati altrimenti. I social media in questo caso hanno portato alla luce forme di altruismo nei giovani, basate su ideali in trasformazione riguardanti l'ambito pubblico.

Si può fare un punto conclusivo con la considerazione che la "busta rossa" su WeChat da una parte e l'Open Access o il Couch Surfing dall'altra rappresentano usi decisamente significativi dei social media per scopi che si collegano allo scambio, al denaro, alla de-mercificazione e al valore. Questi sono in ogni caso temi più generali dell'idea tradizionale di commercio. Una delle nostre conclusioni è che per quanto riguarda il lavoro e il commercio, come per tutte le altre questioni che solleviamo, i social media entrano in questa più ampia contestualizzazione, con riferimento particolare alle relazioni sociali che sono il principale contenuto dei social.

Una seconda conclusione è che se il fattore primario dell'uso dei social media nel commercio fosse stata la razionalità economica formale allora avremmo previsto una relativa omogeneità nell'impatto dei social media nei diversi campi di ricerca - almeno quelli a un livello simile di sviluppo economico e con storie e forme di capitalismo mercantile simile. Tuttavia non è stato così. Piuttosto, nel sud Italia il commercio era visto come qualcosa che avrebbe potuto espandere positivamente le relazioni sociali e la socialità; essere dentro il commercio era parte dell'essere sociale, pubblico e visibile. $\mathrm{Al}$ contrario, in Inghilterra abbiamo trovato che le persone preferiscono un e-commerce astratto e impersonale. Solo transazioni molto specifiche chiamano in causa l'elemento personale nella loro relazione con i clienti, mentre il principale impatto della nuova pubblicità è visto come una violazione di una privacy tenuta in alta considerazione.

Quindi il punto di questa parte finale è ricordare a noi stessi che il modo in cui i social media impattano sul commercio dipende da un insieme più vasto di valori - per esempio, quanto il denaro o gli affari sono intesi in alcuni posti come parte integrante, mentre in altri posti come opposti, alle relazioni sociali appropriate e private. La generalizzazione, che sembra resi- 
stere a questa contrapposizione e per questo è la nostra terza conclusione, è che nel complesso le persone associano i social media e il commercio per lo più quando quel commercio segue la comunicazione di scala ristretta, personale e di gruppo. 



\section{Capitolo 7 \\ Relazioni online e offline}

Con l'aumentare della popolarità e dell'ubiquità dei social media in tutto il mondo si fa strada la convinzione che ci sia una nuova generazione di cosiddetti 'nativi digitali' ${ }^{1}$, nati e cresciuti nell'era digitale. I social media sembrano pronti a diventare un fondamento, in costante crescita, di molte delle relazioni quotidiane. Di conseguenza, gran parte del mondo si sta sforzando di dare senso a questo nuovo fenomeno e al suo impatto. Proprio perché i social media sono oggi così incastrati nelle vite dei più giovani, viene fuori il timore che stiano sostituendo le interazioni offline con quelle online.

Un confronto fra i due tipi di relazioni indicati come online e offline potrebbe tuttavia implicare pure che si tratti di tipologie mutualmente esclusive o opposte. Attraverso tutta la nostra ricerca, però, abbiamo inteso le relazioni come create, sviluppate e alimentate mediante l'interazione integrata online e offline. L'intero ambito delle relazioni offline, dalla famiglia, alla scuola, al lavoro, alle relazioni sociali entro il vicinato più ampio, può anche presentarsi online in una maniera che raramente è separata dalla vita offline di ciascuno. La percezione comune delle relazioni online come qualcosa in contrasto con la 'vita reale' - popolata dalle proprie relazioni offline, più autentiche o reali - sembra pertanto semplicistica e foriera di fraintendimenti. Essa corrisponde a una iniziale critica del concetto di 'virtuale', un termine in evidenza durante il periodo inziale dell'uso di internet ${ }^{2}$. In breve, il nostro studio considera i social media pressoché nello stesso modo in cui ciascuno di noi considera la linea telefonica fissa, oggi mai descritta come un aspetto della vita, 'online/al telefono', separato.

È tuttavia essenziale, per noi in quanto ricercatori, riconoscere che per quanto si possano avere dei dubbi, come accademici, su questa terminologia dualistica, essa resta una modalità primaria secondo cui le persone in tutto il mondo intendono i media digitali e ne fanno esperienza. I nostri informatori davvero parlano costantemente di un mondo online separato. Inoltre, è necessario tener conto del fatto che le persone danno significati diversi ai termini 'online' e 'offline'. Per esempio, nel sito in India del sud, quando interrogate sulla privacy delle fotografie, molte persone rispondevano: "non le condividerò online, solo offline”. Come notato da Venkatraman, per 'of- 
fline' le persone di fatto intendono l'invio di foto ai loro amici più stretti via WhatsApp. Tecnicamente WhatsApp è 'online' nel senso che trasmette tramite un'app dello smartphone, ma è 'offline' per l'idea che ne hanno queste persone dal momento che per loro non si tratta di 'internet'. 'Offline' si riferisce anche alla natura strettamente privata della socialità, laddove online è inteso come la faccia pubblica di internet. Gli stessi informatori, però, in altri contesti si riferiscono a WhattsApp come un social media e dunque online. Pertanto, anche se vogliamo rispettare il fatto che i partecipanti al nostro lavoro di ricerca usano comunemente i termini online e offline, sia l'uso che ne fanno loro sia quello che ne facciamo noi sono spesso discordanti.

In questo capitolo innanzitutto avremo a che fare con il timore diffuso che la crescita della mediazione digitale conduca a relazioni meno autentiche di quelle offline, una convinzione che può portare le persone a considerare le società umane come qualcosa che sta diventando meno reale, dato che le relazioni sono mediate dalle tecnologie digitali. Il capitolo poi si sposterà verso il nostro approccio specifico, che esamina la socialità nel tempo dei social media tramite le lenti dell'etnografia. La parte finale del capitolo esplorerà ulteriormente le nuove possibilità di esperienza umana e di relazioni sociali in seguito all'uso globale dei social media in contesti diversi.

\section{'Autenticità' e 'mediazione': il grande dilemma}

Perché le persone sentono che usare le tecnologie digitali faccia perdere loro qualcosa di sé? Certo non è la prima volta che le società hanno temuto di perdere l'umanità nel fronteggiare una nuova tecnologia ${ }^{3}$. Come già indicato, i timori possono essere riportati indietro a quando fu adottata nell'Antica Grecia la scrittura. Socrate (come racconta Platone) mise in guardia le persone circa la minaccia che questa nuova tecnologia rappresentava per le tradizioni orali della società greca; secondo lui scrivere avrebbe creato l'oblio ${ }^{4}$. Ironia della sorte, senza la scrittura le osservazioni di Socrate avrebbero potuto restare a lungo dimenticate.

Nessuno oggi pensa che la scrittura ci renda meno veri o umani. Al contrario, l'analfabetismo può essere visto come mancanza di una capacità fondamentale che ogni essere umano dovrebbe possedere. Più familiare potrebbe suonare la critica che la televisione ci riduce a "vegetali". Epperò l'idea della famiglia riunita insieme a guardare la televisione è diventata oggetto 
di tenera nostalgia, a confronto con ciò che è percepito come il dominio dell'interazione digitale, più individualizzato e ancor meno reale 5 . Anche oggi troviamo accuse che evocano quelle di Socrate. Fra queste c'è l'idea che la tecnologia digitale riduca la nostra capacità di pensare, abbassando la nostra soglia di attenzione poiché funzioni cognitive come la memoria sono delegate ai nostri dispositivi digitali ${ }^{6}$.

In tutti questi casi i dispositivi digitali sono considerati come forme di incremento della mediazione che conducono a una perdita di autenticità. In ogni caso, gli antropologi rifiutano l'idea di un'autenticità senza mediazioni, e considerano tutti gli aspetti dell'identità e della relazione come intrinsecamente mediati dalle norme culturali e sociali, inclusi il genere e l'etnicità . È un assioma per l'antropologia il fatto che una società tribale non è meno immersa nella mediazione di una società metropolitana. Quando ci incontriamo, la comunicazione faccia a faccia è completamente mediata da convenzioni e etichette che riguardano il comportamento appropriato fra i partecipanti alla conversazione. Le regole della parentela possono limitare ciò che è permesso dire in modo altrettanto efficace dei limiti tecnologici. Per gli antropologi, quindi, comunicare online si può considerare come un passaggio nella mediazione culturale, ma non rende una relazione più mediata.

C'è il rischio che il nostro timore sulla nuova tecnologia ci porti a negare semplicisticamente la natura mediata della precedente socialità offline, considerata non solo come più vera, ma anche come 'più naturale'. Al contrario, in quanto antropologi intendiamo usare lo studio dei social media per approfondire la nostra valutazione della natura mediata della precedente socialità offline. Per esempio, c'è un uso crescente di webcam nella modalità 'always-on', così che coppie e altre persone che vivono in luoghi diversi possano sentirsi come se vivessero ancora insieme. Studiando queste pratiche possiamo arrivare a comprendere le forme di interazione, evitamento e silenzio adottate dalle persone in precedenza, quando vivevano insieme nello stesso spazio domestico, ma avendo imparato a rispettare l'autonomia reciproca e a creare momenti comuni e forme di interazione ${ }^{8}$.

Nel sito di Spyer in Brasile le persone usano il termine "amici di Facebook" per riferirsi a conoscenti con i quali si è in contatto solo attraverso i social media. In molti casi, non solo in Brasile, i cosiddetti "amici di Facebook" maturano l'implicazione che l'"amicizia" di quel tipo, anche se va sotto l'etichetta di "amici", è una categoria minore di amicizia rispetto a "veri amici". In Cina le persone usano 'jiangshi' ('zombie') o 'shiti' ('salma') per descrivere 
quei contatti sui social media con cui non si è mai avuto alcun tipo di comunicazione (nessun "mi piace", nessun commento e nessun messaggio privato o di gruppo); non sono nemmeno considerati come esseri umani viventi. Tali fenomeni sono stati osservati anche in altri siti. Ai fini dell'analisi accademica, tuttavia, è necessario riconoscere che le persone hanno sempre posto in questione le loro amicizie offline per determinare se si trattasse di "veri" amici e per stabilire quanto ci si potesse fidare di loro o farvi affidamento. Benché la terminologia faccia pensare a una semplice opposizione fra amicizia online e offline, quindi, queste domande sull'amicizia si sono in gran parte trasferite dalle relazioni offline a quelle online. Possiamo però ancora riconoscere che relativamente a questa esperienza di amicizia solo-online, e ai modi in cui usiamo queste relazioni, potrebbero esserci aspetti inediti.

Abbiamo scoperto che in gran parte dei posti le persone ormai si aspettano che ci sia concordanza fra i due ambiti. Se i brasiliani hanno relazioni cordiali offline, è probabile che anche sui social media abbiano relazioni affabili. Per un buon amico, è probabile che i social media siano di ausilio per coltivare e rilanciare l'amicizia, mentre se non c'è legame in prima istanza, essere amici su Facebook può fare ben poca differenza rispetto alla relazione offline. Nella gran parte dei casi i social media possono essere uno spazio dove gli amici di amici e di parenti si trasformano in propri amici. Questo è apparso particolarmente importante nelle regioni dell'America Latina e a Trinidad, e molto probabilmente riflette il modo in cui l'amicizia era intesa già prima. Da una parte, l'amicizia online potrebbe essere intesa come priva di tutti gli usi sociali ${ }^{9}$ presenti nell'interazione faccia a faccia. Dall'altra, sin dall'inizio di internet le persone hanno usato l'anonimato online per discutere temi che avrebbero potuto trovare difficile condividere con persone che li conoscevano offline. Per questo le persone con cui si condividono i segreti più intimi ora possono essere estranei online che non si conosceranno mai offline.

\section{'Cornici' e 'gruppi': approcci per comprendere la socialità}

Come possiamo allora, nell'era dei social media, trovare più senso nelle relazioni sociali? Anche il concetto teorico di "framing", che deriva dall'analisi di Goffman ${ }^{10}$ dell'azione sociale, è utile per pensare la relazione fra l'online e l'offline. Semplificando, il framing aiuta a posizionare il confine, con la defi- 
nizione delle regole e delle aspettative che guidano il comportamento. Stare dentro il "frame" di un teatro, per esempio, ti dice che dovresti applaudire alla fine e non correre sul palcoscenico a salvare l'eroina. Ci sono miriadi di frame invisibili ma davvero concreti nella vita sociale, e la conoscenza che ne abbiamo ci aiuta a comportarci appropriatamente, secondo le aspettative sociali. Per esempio, il genere di conversazione che si svolge in un pub inglese è diverso da quello di una riunione di lavoro perché il 'pub' e l'ufficio' incorniciano ('frame') differentemente tali comportamenti, anche se si tratta sempre di forme di parlato. Pertanto, dovremmo considerare l'offline e l'online anche come due "frame" nella nostra quotidianità ${ }^{11}$ che possono far scattare atteggiamenti e comportamenti differenti. Questo è anche il motivo per cui, in alcuni casi, le persone sentono che l'ambiente online induce aspetti diversi di certe relazioni. Invece di opporli, possiamo considerare questi contesti come frame complementari che si combinano a fornire una rappresentazione più completa della persona e delle sue relazioni.

In pratica, piattaforme diverse di social media, o gruppi e account diversi dentro la medesima piattaforma, funzionano come "sotto frame", consentendo agli individui con le abilità necessarie di localizzare una nicchia dentro cui confrontarsi con varie relazioni sociali ${ }^{12}$. Per esempio a Mardin, il sito della Turchia sud orientale, è normale trovare teenager e giovani adulti con account multipli sulla stessa piattaforma di social media (per esempio Facebook); possono comportarsi, e di fatto lo fanno, in modo differente a seconda dei gruppi con cui le condividono. Costa mostra come le persone passino agevolmente e velocemente da una piattaforma a un'altra, o tra diversi account all'interno della stessa piattaforma.

Piattaforme diverse o account multipli permettono agli utenti di collocare i loro contatti in categorie diverse come "compagni di classe", "colleghi", e "altri", un modo utile di utilizzare i social media per "separare" i propri network sociali. Anche queste "categorie" sociali organizzate sui social media esistevano nella vita sociale offline da molto prima che arrivassero i social media; ma i social media possono rendere quelle categorie più esplicite. Nel nostro sito nel sud dell'India, per questo, le persone possono trattenere l'assetto di default su Facebook, in modo che gli utenti non siano esplicitamente categorizzati, ma sanno molto bene quali contatti sono della stessa casta, ancora uno dei sistemi di classificazione principali della nazione.

Diversamente da Facebook, Twitter e Instagram, ma come Google +, la piattaforma di social media cinese QQ caratterizza in modo evidente catego- 
rie di amici. QQ incoraggia i suoi utenti a separare i contatti online in gruppi, per esempio "colleghi”, "amici speciali", "amici”, "parenti”, "estranei”, "blacklist" (usata per bloccare i contatti), e qualsiasi altro gruppo personale che si desideri creare ${ }^{13}$. In ogni caso, questi sono visibili solo al singolo utente, non ai suoi contatti. Un'espressione popolare cinese è "conoscerai meglio le tue relazioni se saprai in quale categoria i tuoi amici ti hanno messo nel loro QQ". Tali pratiche sembrano naturali in Cina dove (come sarebbe vero anche di molti altri posti fuori dalla Cina) la socialità è stata a lungo caratterizzata da diversi circoli e gruppi in sovrapposizione ${ }^{14}$. Il principale problema è che le categorie online non possono corrispondere alla complessità delle reti tradizionali di "guanxi" ("relazioni sociali”). Il motivo è che su QQ le categorie di amicizia sono mutualmente esclusive, mentre nella vita offline si può benissimo pensare dei propri amici come trasversali a diversi gruppi. Qui, come in tutti i siti, quindi, i social media possono rafforzare la categorizzazione sociale ma non possono corrispondere alla complessità di questa pratica nel mondo offline ${ }^{15}$.

\section{Dalla vicinanza all'anonimato: la socialità modulabile ${ }^{16}$}

Nel Capitolo 1 si è sostenuto che la forma particolare assunta tramite la polimedialità dentro i social media può essere definita "socialità modulabile". L'esempio iniziale tratto dal sito inglese si basava sugli studenti. Nel nostro sito in Brasile molte persone più grandi hanno accesso ridotto a internet per via della loro scarsa istruzione e per fattori economici, per cui i social media possono essere interpretati come lo spazio dove una persona giovane può creare comunità di pari e far mostra di modernità sfuggendo allo sguardo adulto. Qui, come altrove, i social media spesso diventano un posto per definire relazioni intime (legami di parentela, di stretta amicizia, di coppia), dove a essere modulata, sperimentata, conservata e rafforzata è la stessa relativa intimità. Nel sito dell'India del sud i social media funzionano come "parenti fittizi", molte persone vengono viste come "zii" o "cugini" (anche se non legate biologicamente) in linea con la tradizione delle famiglie estese e delle caste. Così, la parentela si usa come idioma per esprimere la distanza percorsa da una persona lungo questa stessa scala di intimità.

Nel sito di Sinanan a Trinidad molte famiglie hanno genitori, figli o cugini che vivono all'estero, e l'uso dei social media è vitale per il mantenimento 
della relazioni familiari di base. In alcuni dei nostri siti infatti i social media hanno anche consentito a famiglie che non vivono nello stesso circondario di costruire maggiori legami emotivi; possono trascorrere più tempo insieme online di quanto non farebbero se vivessero insieme. Per esempio, i teenager in molti paesi occidentali magari vivono a casa, ma a volte con una relazione davvero ridotta con la famiglia, anche scrivendo avvisi che vietano ai genitori di entrare in camera loro. Gran parte della loro socialità si dipana dagli schermi nelle loro camere, uno spazio tenuto nettamente separato dal resto della casa. Anche se vivono nella stessa casa insieme al resto della famiglia, quindi, si può affermare che questi teenager non vivono "insieme", non più di quanto non vivano "insieme" con le persone con cui socializzano online.

Haynes nel sito nel Cile del nord ha scoperto che WhatsApp è diventato incredibilmente importante per i minatori durante gli estenuanti periodi di separazione dalle famiglie mentre stanno lavorando lontano. E tuttavia, anche se per molte famiglie i social media risolvono i problemi di separazione, per altre possono anche arrecare problemi. Alcuni minatori hanno raccontato che i social media aumentano il sentimento di gelosia fra i partner maschili delle (poche) donne minatrici; in altri casi possono esacerbare relazioni familiari già problematiche. Haynes però riferisce pure che i minatori apprezzano i social media anche per il fatto di portare un elemento del mondo esterno, quindi un senso di umanità, al "manicomio" della miniera.

Nel sito della Cina industriale alcune coppie che hanno mantenuto una relazione reciproca a lunga distanza trovavano persino che i loro partner on line apparissero più premurosi, liberi dalle banali preoccupazioni della condivisione quotidiana. Alcuni membri giovani raccontavano anche che avevano trovato i familiari più grandi più sciolti e divertenti online, grazie all'uso che facevano di "emojis" carini sui social media, mentre negli incontri faccia a faccia si riteneva che queste relazioni dovessero essere più serie e deferenti ${ }^{17}$. Ancora una volta, lungi dall'essere separate dalle relazioni offline, i social media sono diventati una modalità prevalente con cui, in diversi casi, le relazioni tradizionali fra parenti stretti vengono mantenute, conservate, rinvigorite e anche, in certi casi, trasformate. In tutti questi casi la socialità modulabile include la possibilità che le relazioni sui social media siano più intense e intime di quelle offline.

Un altro motivo per cui tutto questo può diventare altamente significativo è qualora le persone siano impossibilitate e muoversi. Miller include nel suo lavoro sul campo uno studio estensivo del modo in cui un ospizio comunica 
con i pazienti, che in gran parte sono malati terminali a causa di un tumore a uno stadio avanzato. Egli ha scoperto che quel tipo di immobilità ha avuto un impatto forte anche in un'area rurale per via della particolare natura della socialità inglese. La socialità nel paese segue un modello generale per cui le persone sono molto socievoli in pubblico, ma fortemente sulla difensiva per quanto riguarda la sfera privata; questo si combina con una forte sensibilità fra le persone più anziane che non vogliono diventare un fardello sulle spalle dei loro parenti e amici. Ne consegue un sorprendente grado di isolamento e solitudine. Come esito di un progetto applicato, ciò ha portato a raccomandare all'ospizio di incoraggiare l'uso dei social media e di dispositivi di facile utilizzo come l'ipad, in particolare nelle fasi iniziali della malattia in cui le persone sono più disponibili a essere supportate e assistite. Avendo stabilito che la causa della solitudine è il dualismo fra socialità pubblica e privata, la definizione specifica dei social media, come forma di socialità modulabile che fa da ponte fra privato e pubblico, li rende una parte molto appropriata di una possibile soluzione al problema della solitudine nel contesto della riservatezza sociale inglese ${ }^{18}$.

In alcuni casi, più stretta è la relazione più sono supportate forme diverse di piattaforme e tecnologie per la comunicazione. Lo studio di Broadbent ${ }^{19}$ mostra che, nella regione dove ha lavorato (la Svizzera), la proliferazione di social media non porta necessariamente all'estensione di nuove connessioni sociali "ma piuttosto all'intensificazione di un piccolo gruppo di relazioni molto intime che ora sono gestite facendo coincidere la ricchezza della loro connessione sociale con quella di canali di comunicazione paralleli" ${ }^{20}$. Come accede spesso con il nostro progetto, questa generalizzazione funziona solo in certe regioni e non in altre.

La socialità modulabile può essere messa in relazione con molte altre dimensioni, per esempio il grado in cui le persone hanno bisogno, o credono di aver bisogno, del tipo di relazioni sociali a cui possono avere accesso attraverso i social. Nicolescu, nel sito in Italia del sud, ha rilevato che molti abitanti consideravano le loro precedenti reti sociali stabili e sufficienti. Dal momento che la maggioranza dei contatti Facebook riguarda persone della stessa città, il fatto di risiedere nello stesso posto comportava che ci fosse scarsa necessità dei social media come mezzo per mantenere queste relazioni. Di conseguenza, molte persone ritenevano i social media sostanzialmente irrilevanti per la loro vita. Nicolescu tuttavia ha notato anche che per un piccolo gruppo di persone che aveva conseguito un livello di istruzione 
più alto in grandi città e aveva reti sociali molto più diversificate fuori dal piccolo centro, ritornare in paese significava spesso la perdita di queste connessioni sociali estese. Per loro, i social media giocavano un ruolo vitale per il mantenimento di queste connessioni più ampie, considerate spesso più importanti delle relazioni quotidiane offline.

In generale, come si rileva nella nostra survey del Capitolo 4, le persone considerano positivamente il modo in cui i social media facilitano le relazioni sociali ${ }^{21}$. Per le relazioni più strette essi di solito sono un luogo in cui vengono coltivati e espressi fiducia e affetto. Nello stesso tempo, le persone sono consapevoli dell'altra faccia della medaglia, ossia il fatto che la maggiore visibilità delle relazioni porta alla gelosia e al controllo.

A confronto con questi più comuni atteggiamenti riguardo all'uso dei social media in riferimento alle relazioni sociali intime, si osserva una reazione molto diversa riguardo alla loro potenzialità in fatto di contatti con estranei.

I cileni tendono a non usare siti web per incontri o app come Tinder e Grindr, sempre più popolari invece in Inghilterra; qui le persone a volte sembrano avere meno sfiducia nei confronti degli estranei come potenziali partner sessuali piuttosto che come persone che potrebbero vedere ciò che postano sui social media. Per cercare relazioni d'amore online i cileni fanno invece affidamento sugli amici degli amici su Facebook. In entrambi i siti gli estranei non diventeranno contatti online senza una qualche forma di approvazione offline, per esempio conoscere la persona offline o conoscere qualcuno che la conosce bene ${ }^{22}$. Altrimenti gli estranei sono oggetto di sospetto.

Questo contrasta con il nostro sito nel Cile rurale, dove McDonald rileva che precedentemente all'uso dei social media le interazioni con "mosheng ren" ('estranei') erano in genere molto meno abituali. Adesso i giovani possono fare amicizia con 'luanjia' ('un grande numero di estranei) in modo casuale, anche se altri, specialmente le coppie sposate, nei confronti degli estranei on line mantengono un atteggiamento di cautela ${ }^{23}$. Analogamente, Costa ha trovato che a Mardin i social media, specialmente Facebook, sono stati usati per allargare le reti sociali di un individuo agli 'yabanci' ('estranei'), mentre tradizionalmente le reti sociali delle persone riguardavano per lo più sia 'akraba' ('parenti') sia 'komsu' ('vicini'), e per determinate categorie di persone anche 'arkadas' ('amici') 24 .

Anche nel sito della Cina industriale è stata osservata una trasformazione degli atteggiamenti nei confronti degli estranei. Qui molti considerano 
l'amicizia che è solo online come una relazione 'chun' ('più pura'), dato che non comporta le richieste pratiche che spesso caratterizzano pesantemente le relazioni offline ed è meno infusa di gerarchia sociale. Per come la vedeva Feige, un operaio:

A loro [gli amici online] piaci e conversano con te perché veramente ti apprezzano per come sei, non perché sei ricco e quindi possono chiederti denaro in prestito, o perché sei potente e possono ottenere un lavoro. Qui [online] ogni cosa è più pura, senza potere e denaro di mezzo.

Analogo sentimento è stato espresso da alcuni proprietari di fabbriche. Dicevano che certe volte evitavano di partecipare alle rimpatriate scolastiche nel timore di richieste di aiuto finanziario da parte dei vecchi compagni di classe. Tuttavia, parlavano volentieri online con estranei su WeChat per scaricare lo stress che secondo loro non bisognava mostrare ai loro subordinati o ai familiari. Benché, in questo sito, i proprietari e gli operai rappresentino i due redditi estremi, essi esprimono motivazioni simili riguardo all'amicizia e al contatto online con estranei. In entrambi i casi le relazioni online sono considerate più autentiche di quelle offline, che in molti casi sono molto mediate (o 'inquinate', come dicono) da fattori quali ricchezza e status sociale.

Nel caso di social media più pubblici, l'esposizione online di relazioni va anche interpretata come una "verifica ufficiale". Nel nostro sito in India del sud, le comunicazioni relativamente strette e intime favorite da WhatsApp si usavano per comunicazioni fra membri della famiglia. D'altro canto, Facebook, essendo la piattaforma più pubblica, è il posto dove i legami familiari e parentali sono messi consapevolmente in scena di fronte a un pubblico di contatti non familiari. Per esempio, un aggiornamento di Facebook per la nascita di un bambino di solito è seguito da molti "mi piace" e commenti da parte dei familiari, anche se tutti loro, prima di postare, hanno già fatto auguri e congratulazioni al telefono o di persona.

Queste performance sui social media sono fatte su misura per chi non fa parte della famiglia e per il più ampio pubblico che condivide Facebook. Haynes spiega che nel Cile del nord una relazione d'amore sarà riconosciuta "ufficialmente" solo quando sarà pubblicata su Facebook come "aggiorna relazione" o postando informalmente note d'amore sulla bacheca "altre informazioni significative". Analogamente, nella Cina industriale le giovani coppie hanno costante bisogno del pubblico online su QQ a testimonianza 
della loro storia d'amore. In alcuni casi, giovani operai migranti arrivano a formare gruppi su QQ con tutti i loro contatti online per dire "ti amo" in pubblico (sulla chat del gruppo online) al/alla loro fidanzato/a. Queste dichiarazioni online sono considerate una delle assunzioni di impegno più grandi in una relazione. Così, i social media ci rendono più consapevoli e auto-consapevoli sia delle nostre relazioni sia di noi stessi. In questi casi, le relazioni offline, per essere accettate come "vere", dipendono dal loro riconoscimento online.

Se mettiamo insieme questi esempi, vediamo che, in modo trasversale a tutti i siti, i social media hanno conseguenze del tutto contraddittorie, in parte anche perché con "social media" ci si riferisce a un ampio insieme di piattaforme. Se li consideriamo come polimediali, vale a dire come una gamma entro cui ognuno ha la propria nicchia complementare, allora vien fuori un po' più di consistenza. C'è l'uso di piattaforme per creare un opposizione fra socialità più pubblica e più privata, così come l'utilizzo sia di piattaforme diverse sia di usi diversi della stessa piattaforma, per poter organizzare il proprio mondo sociale in categorie separate. Ciò include $i$ diversi atteggiamenti degli insegnanti dell'India del sud verso WhatsApp, a seconda che ci si rivolga alla scuola o alla famiglia, l'uso di Facebook da parte dei teenager brasiliani per liberarsi dal controllo degli adulti o degli amanti adulti cileni per ottenere conferma ufficiale dal pubblico più ampio. Dalla possibilità di mantenere relazioni intime a quella di creare relazioni con gli estranei, i social media si possono considerare come una forma di "socialità modulabile" che mette le persone in grado di esercitare un miglior controllo sulla loro vita sociale. Questo si può fare adattando le norme sociali esistenti a contesti differenti o creando forme interamente nuove di relazioni sociali e di socialità sfruttando la possibilità di graduare prossimità e distanza.

\section{Identità online: dimensioni nuove e allargate della vita quotidiana}

Questo ci porta al nostro ultimo tema relativo all'identità online: la nostra tendenza a considerare l'identità come costruita attraverso l'interazione sociale e non come una condizione psicologica data. Il lavoro di Sennett ${ }^{25}$ mostra come l'identità un tempo fosse quasi del tutto ascritta. Una persona nasceva dentro una particolare classe, ambito d'occupazione, ruolo sociale e luogo d'origine e qualsiasi cosa accadesse, eri destinato a restare un macel- 
laio della Bretagna per tutta la vita. Giddens ${ }^{26}$ sostiene che nel mondo moderno, d'altra parte, le persone normalmente hanno a che fare con elementi identitari che non sono più fissi e stabili, ma riflettono le dinamiche delle situazioni in cui si trovano, fino al punto che oggi si combatte per preservare una narrazione coerente della propria identità. Entrambe le prospettive sono completate da Goffman ${ }^{27}$, il cui già citato concetto di framing riguarda anche il modo in cui le persone gestiscono le identità multiple legate ai vari ruoli e contesti sociali: madre, lavoratrice, atleta. Andare online aggiunge ulteriori frame a questo insieme. Quando questa socialità si fa più modulabile, può anche dotare l'identità online di una maggior flessibilità.

Si può analizzare tutto ciò attraverso le conseguenze della maggiore visibilità della persona e delle relazioni ${ }^{28}$. Questo è particolarmente importante a Trinidad, dove si è già sviluppata una associazione fondamentale con la costruzione del sé come progetto che può essere messo in atto solo attraverso lo specchio della visibilità sociale. In breve, gli abitanti di Trinidad riconoscono che la verità di una persona non è ciò che pensa di essere, ma ciò che gli altri ritengono sia sulla base del modo di apparire. Storicamente questo è emerso dai movimenti antischiavisti e di resistenza. Invece che avere uno status ascritto, nella schiavitù o nella gerarchia sociale, gli individui potevano tentare di creare una persona del tutto diversa agli occhi del pubblico attraverso una mascherata. Idealmente le persone dovrebbero essere giudicate non per la loro nascita o il loro lavoro, ma per la loro abilità di creare se stesse come affascinanti o potenti. Il modo in cui cammini e parli e ti vesti non è un'illusione o una semplice performance: è il solo modo giusto per cui potresti essere giudicato.

La stessa logica funziona per Facebook. Immagini come fotografie e memi dominano i post dei trinidadiani perché ciò che viene messo in mostra sui social media è la verità, curata, che si vuole raccontare agli altri. Attraverso questa cura essi si mostrano come internazionali e cosmopoliti, o come dediti alla famiglia, o come 'gangsta' (membro di una gang). A Trinidad i social media rendono iper-visibile ciò che di una persona è già visibile, rafforzando ulteriormente la sua identità costruita. Anche per questo Miller ha indicato Facebook a Trinidad come "The Book of Truth"29.

L'uso trinidadiano di Facebook forse è un esempio estremo di come i social media sono diventati un luogo in cui le persone creano un'identità visibile che può essere considerata più vera della loro persona offline. Un altro esempio sarebbe l'uso di QQ fra gli operai migranti cinesi, anche se in questo 
caso il risultato è totalmente invisibile al mondo offline. Nel sito della Cina industriale decine di migliaia di operai provengono da villaggi dell'interno del paese. Nel contesto dello sviluppo dell'economia cinese, migrano nelle città industriali in cerca di lavoro che si riduce in gran parte nel completare i compiti già svolti dalle macchine. I lavoratori migranti, chiamati 'liudong renkou' ('popolazione fluttuante') sono costantemente in movimento per procurarsi lavoro temporaneo e mal pagato. Non sono qualificati per ottenere miglioramenti economici e formativi destinati ai residenti urbani più stabili e tendono a vivere e lavorare in condizioni estremamente al di sotto degli standard. È la loro vita offline che questi lavoratori vedono come temporanea. Al contrario, considerano i social media come un posto molto più stabile e permanente dove possono tenere i contatti con la famiglia e con gli amici. Postando "foto fantastiche" (per esempio auto di lusso, vacanze romantiche, immagini di donne come principesse) e immagini 'feizhuliu' ('anti-mainstream'; per esempio fotografie di rocker ribelli), o condividendo 'zuppa di pollo per l'anima' (storie per trovare ispirazione, del tipo 'sei povero perché non sei abbastanza ambizioso') ${ }^{30}$, le persone costruiscono di fatto la loro identità online. Si tratta di qualcosa di molto diverso dallo stigma di 'di suzhi' ('bassa qualità umana') stampata addosso agli operai migranti cinesi offline.

Come indicato in altre ricerche $e^{31}$, le persone che hanno difficoltà nell'esprimere o "essere" se stesse nelle interazioni faccia a faccia molto più facilmente si costruiranno ciò che considerano come il "vero sé" e stabiliranno relazioni più strette con le persone che incontrano online. Come ci ha detto una tipica giovane operaia cinese che ha abbandonato la scuola, 'la vita fuori dal cellulare è insopportabile'. Potremmo essere tentati di ignorare le immagini di nozze perfette o di essere una principessa considerandole come mere fantasie. In questo caso, però, la vera ragione per assumersi questo compito è fornire un percorso verso una diversa modernità, di cui queste persone cercano per se stesse di immaginare la natura. Lo spazio per farlo offline è estremamente ristretto. $\grave{E}$ online dove le persone forgiano e mettono in atto una versione più permanente di se stesse, un'immagine che sperano nel tempo di riuscire a ottenere, grazie a maggiori guadagni. Per loro le aspettative, i desideri e la felicità che esprimono e di cui si vestono online sono fortemente reali. In effetti, i lavoratori migranti partecipano di una migrazione doppia ma simultanea. La prima è fisica, dal villaggio alle fabbriche in città; la seconda è dall'esperienza offline a quella online. I social media non sono 
semplicemente una tecnologia per la comunicazione, la fantasia o il divertimento. Per questi migranti i social media sono un posto davvero vissuto. Lavorano offline e vivono online.

\section{Conclusioni}

Questo capitolo è iniziato delineando la preoccupazione diffusa, almeno in alcuni paesi occidentali, che i social media, insieme al presunto aumento di relazioni mediate, siano qualcosa che sta rendendo gli esseri umani meno "veri", o quantomeno più lontani dalle realtà della vita ${ }^{32}$. L'ultimo caso di studio forse rappresentava l'evidenza più profonda del perché non è necessariamente questo il caso. Nel sito della Cina industriale i lavoratori migranti, la cui vita è dominata dal lavoro manuale, fanno esperienza dell'online come il posto il cui possono essere più umani, in grado di esprimere aspirazioni individuali e speranza per il futuro. La relazione tra esseri umani e nuove tecnologie per questi informatori non è ambigua: è essenziale. La discussione nei paesi occidentali dà per scontato cosa sia la vita offline delle persone. Anche in questo caso gli operai dormono, lavorano e mangiano offline, senza che molti di loro abbiano davvero molto di ciò che chiameremmo "vita" offline. Hanno invece una vita online quando non stanno dormendo, lavorando e mangiando, quando possono diventare se stessi e relazionarsi con altri. Si può anche vedere come, in certi casi, denigrare i social media perché inautentici può essere in parte una prassi elitaria. Questi gruppi, certi della loro capacità di costruirsi offline, forse cercano di svalutare i tentativi da parte della popolazione dotata di minor potere di affermare l'autenticità della creazione del loro sé online.

Analogamente, non possiamo ignorare la sensibilità dei trinidadiani, i quali suggeriscono che l'apparenza visibile che si è in grado di creare per se stessi online è proprio per questo più vera di quella su cui non si ha alcun controllo: per esempio, la particolare forma del naso con cui sei nato o la divisa che devi indossare per lavoro. In termini più generali, via via che passiamo attraverso i siti, la situazione si fa meno dualistica. In molti casi, l'online è diventato semplicemente un altro frame di contesto in linea con i molti contesti precedenti della vita offline, dove le persone, sempre, vivono sia al lavoro sia in famiglia durante le vacanze e così via. Quindi, in linea con un'ampia letteratura, che include i lavori di Turkle ${ }^{33}$, Baym ${ }^{34}$, Hampton e 
Wellman $^{35}$, Livingstone ${ }^{36}$ e altri, questo progetto porta prove a favore di un ulteriore richiamo ad andare oltre le argomentazioni semplicistiche, basate su opposizioni quali virtuale $v s$ reale, e a riconoscere invece che molte persone oggi sono impegnate in una molteplicità di comunicazioni e di identità online e offline senza un confine netto fra esse. Allo stesso tempo, va considerato il fatto che quasi tutti i nostri informatori distinguono offline e online, e se ne servono per esprimere un'ampia gamma di opposizioni diverse, usando spesso termini come 'reale' o 'virtuale'. Inoltre, riconosciamo anche che ci sono persone che privilegiano l'online come autentico e genuino, così come coloro che ritengono l'offline la condizione naturale.

La parte centrale di questo capitolo ha suggerito un diverso approccio a questo tema. Invece di partire dall'opposizione offline/online, il nostro progetto ha preso le mosse da una definizione di social media in termini di socialità modulabile. In altre parole, nello spettro delle relazioni delle persone osserviamo costantemente gradazioni possibili, dal più intenso, al meno intimo, al più privato, e così via. Da questa prospettiva la distinzione fra offline e online viene sostituita dalla considerazione delle molte diverse dimensioni in cui graduiamo le nostre relazioni, e quella distinzione diventa uno degli aspetti. Molti dei nostri esempi, infatti, hanno riguardato casi in cui l'online, prendendo per esempio a parametro l'intimità, stava più alto in questa scala rispetto all'offline. Ci sono esempi in cui si ha maggiore apertura verso gli estranei o preferenza ad aprirsi con i pari con cui non si vive e non con i genitori con cui si vive. In generale, tuttavia, il punto non è che o si è più intimi o più distanti. Le persone riconoscono piuttosto di poter utilizzare una varietà di contesti relazionali come frame differenti. Tra questi può accadere che ce ne sia uno solo che è online come opposto a offline. Infine, è possibile che questa nuova dimensione della creatività visibile sia nelle relazioni sia nell'identità ci stia rendendo più consapevoli sia delle nostre relazioni sia di noi stessi. 



\section{Capitolo 8 \\ Genere}

In questo capitolo esamineremo le influenze che i social media hanno avuto sulle relazioni di genere, sulle norme di genere e sulle identità nei nostri siti di ricerca. Per genere intendiamo le differenze, costruite socialmente e culturalmente, tra femminilità e maschilità, modellate da innumerevoli fattori incluso l'uso della tecnologia e dei media digitali. Le prime ricerche su internet ${ }^{1}$ spesso hanno espresso stupore davanti alla peculiarità degli spazi sociali online in cui potevano - o almeno così sembrava - sparire caratteristiche personali come il genere, la razza, l'età e anche tratti fisici evidenti del corpo, dato che si poteva adottare una maschera online totalmente distinta. Le studiose di internet femministe ${ }^{2}$ hanno enfatizzato il ruolo dei media digitali nel fornire strumenti di empowerment che consentono a uomini e donne di rappresentare liberamente i sé e le identità che scelgono per se stessi online, sfuggendo alle norme oppressive di genere del mondo offline.

Donna Haraway ${ }^{3}$ presenta uno degli argomenti più noti a favore di questa tendenza, e enfatizza il potere della tecnologia di trasformare le relazioni di genere e le identità. Inoltre, proprio perché il genere poteva almeno potenzialmente essere cancellato o reso irrilevante online, risultava più evidente che le nozioni di genere fossero costruite culturalmente, create mediante l'interazione tra il mondo sociale e la cultura materiale (e le tecnologie) che ci circonda, tutt'altro quindi che semplici dati biologici ${ }^{4}$. In quel periodo, altri ottimisti digitali ${ }^{5}$ enfatizzavano internet perché rendeva possibile la costituzione di nuove forme di identità di genere individuali e collettive. Secondo loro, internet agevolava l'espressione autentica dell'agency specifica delle donne invece di qualcosa che era loro imposto.

Retrospettivamente, tali nozioni di identità online, libere di fluttuare sganciate dai corpi del mondo vero, si sono rivelate di breve durata. Quando i social media hanno sostituito modalità più anonime di interazione come chat room e forum, le identità offline sono diventate sempre più importanti nel determinare quelle online, in special modo in contesti dove norme sociali e idee di decoro influenzano o controllano le reazioni sociali in modo significativo. Negli anni Novanta, alcuni videro l'online come potenzialmente libero da una rigida identità di genere. Altri, in continuità con una 
discussione che risale agli anni Sessanta e Settanta, tendevano a enfatizzare il ruolo della tecnologia nel riprodurre il dominio maschile. In questo caso, il riconoscimento che le tecnologie digitali possono destabilizzare le differenze di genere ${ }^{6}$ consentì una revisione critica.

Dopo questi decenni, che scivolarono da un'enfasi a un'altra, molte persone oggi riconoscono che la tecnologia crea potenziali, resi manifesti a seconda dei contesti in cui sono inseriti. Alcuni studi più recenti hanno messo a fuoco il modo in cui i social media hanno offerto nuovi strumenti di presentazione del sé e di gestione delle identità di genere, specialmente tra i giovani e i teenager ${ }^{7}$. Altri hanno sottolineato le dinamiche di apertura del sé come elemento principale di questo processo ${ }^{8}$. È rimasto tuttavia poco chiaro il grado di generalizzazione possibile al di fuori del contesto euro-americano in cui sono state svolte le ricerche più importanti.

Questo capitolo presenta esempi etnografici per analizzare i modi in cui i social media talvolta hanno rafforzato le relazioni di genere esistenti e gli ideali dominanti di maschilità e femminilità, ma in altri casi hanno portato a trasformazioni del modo in cui le differenze di genere sono immaginate e messe in atto. L'attenzione alla diversità culturale non solo rimanda alla gamma dei siti, ma anche alla diversità all'interno di ciascuno di essi, in considerazione della variazione regionale, religiosa, di classe, urbana/rurale, e etnica. Il capitolo è diviso in due parti principali. La prima analizza i modi in cui i social media hanno favorito la riproduzione e il rafforzamento delle norme di genere esistenti, mettendo in luce le similarità tra l'online e l'offline. La seconda parte discute alcune delle trasformazioni portate dai social media alle relazioni e alle norme di genere. Il capitolo termina con una breve discussione sulle identità e sulle pratiche sessuali non-eteronormative, inclusi gay, lesbiche, bisessuali e transgender, descrivendo se e in che modo i social media possano rappresentare, per le persone con questo genere di identità, una maggiore opportunità di interazione privata o riservata $\mathrm{o}$, invece, raggiungere una maggior visibilità pubblica.

\section{Continuità: presentazione di genere del sé}

In tutti e nove i siti del nostro studio, i social media hanno riprodotto e rafforzato le norme che regolano le differenze di genere nel mondo offline, ma in modi e in misura diversi. In particolare, la riproduzione degli ideali 
dominanti di femminilità e di maschilità nei social media pubblici ${ }^{9}$ come Facebook è un elemento comune trasversale a quasi tutti. Gli spazi pubblici on line si sono caratterizzati spesso come fortemente conservativi, rafforzando norme di genere consolidate. La costruzione del sé sui social media continua ad avere un aspetto di genere, come una parte delle varie e intersecate identità individuali, proprio come accade nella vita quotidiana offline ${ }^{10}$.

Il sito della Turchia sudorientale illustra forse meglio la natura conservativa delle piattaforme tipo Facebook. Qui gli uomini tendono a rappresentarsi come professionisti di successo, mentre le donne esaltano le loro qualità estetiche, oppure la loro adesione ai valori musulmani di modestia e purezza; tutti omettono quegli aspetti della loro vita che non riflettono i valori egemoni sull'onore femminile e maschile e l'etica musulmana. Per esempio, raramente sono rappresentate interazioni fra donne e uomini in quegli spazi di Facebook accessibili al più ampio pubblico di amici e parenti. Anche se sono normali le conversazioni fra giovani uomini e donne in ambiti secolari e misti come la scuola e l'università, questo non comparirà online in modo evidente. È raro che venga rappresentata anche l'occasione di incontro di gruppi misti di parenti per bere caffè o tè insieme in spazi semi-pubblici come bar e ristoranti, dato che potenzialmente potrebbero dare adito a pettegolezzi e fraintendimenti.

Le donne laiche e istruite che sono fidanzate ufficialmente non renderanno mai pubblico questo fatto sui social media. Un esempio è il caso di Leyla. Se qualcuna delle sue amiche dovesse scattarle delle fotografie insieme al suo fidanzato, lei è sempre molto attenta ad assicurarsi che nessuno posterà queste fotografie sulla sua bacheca di Facebook. Fortunatamente, prima di postare foto su Facebook le persone di solito chiedono il permesso. Su Facebook separazione di genere e conformità sono quindi anche più rigide ed estreme di quanto non siano offline, semplicemente perché questo spazio è soggetto a controllo continuo, in una misura molto maggiore della maggior parte degli spazi offline. È consueto per i giovani, donne e uomini, ricevere telefonate dai loro parenti più grandi con richieste di chiarimenti e di giustificazione in relazione a immagini specifiche che hanno visto su Facbook. Le persone trascorrono molto tempo controllando e speculando sulle bacheche dei loro amici di Facebook. Qualsiasi deviazione dalle norme può divenire oggetto di pettegolezzi e maldicenze, che potrebbero anche portare alla vergogna e alla rovina della reputazione individuale e familiare.

Un giovane di nome Fatih partecipava a un evento in un locale insieme a 
due ospiti speciali: DJ donne provenienti da Istanbul che dovevano esibirsi quella sera. Le donne avevano un abbigliamento che appariva del tutto indecoroso secondo gli standard locali. Anche se lui e i suoi amici trascorsero l'intera serata a scattare foto delle due donne, nessuno postò alcuna di quelle foto su Facebook. Sullo spazio online visibile a centinaia di amici e parenti questi uomini si conformarono strettamente alle norme morali che impongono una condotta virtuosa e pura.

Gli ideali dominanti di femminilità vengono riprodotti anche mediante una presenza di corpi femminili online molto contenuta: donne giovani non sposate di ambiente più tradizionale usano spesso Facebook con foto del profilo false e con nomi falsi per evitare di essere viste dai parenti maschi più anziani. Così facendo riproducono le norme sociali in vigore che per tradizione vietano loro di mostrarsi in spazi pubblici; però, nello stesso tempo questo produce possibilità nuove di trasgredire queste norme, tenendo segretamente uno spazio di rappresentazione fuori dal controllo della famiglia.

Lesclusione parziale delle donne dagli spazi più pubblici dei social media non è limitata alla Turchia sudorientale. Nel nostro sito nella Cina rurale, diverse donne sposatesi di recente hanno cancellato molti estranei dalla loro lista di contatti, arrivati in seguito a una pregressa frenesia di "aggiungi" prima del matrimonio. Altre possono anche smettere di usare i social media del tutto. Spesso le donne incinta cancellano molte persone e si rendono meno visibili sui social, mettendo in scena una transizione dalla vita pubblica verso l'istituzione privata della maternità. La perdita di visibilità delle donne sui social media quindi aumenta con il passaggio a certe fasi della vita. Però, viceversa, le donne sposate sono particolarmente attive e visibili su spazi pubblici offline, come in gruppi di danza organizzati.

In questo caso è la connotazione dell'online come spazio immorale a scoraggiare alcune donne da una esplicita attività online. Le donne che sono attive online usano moltissimo il maggiore anonimato consentito dai social media cinesi ${ }^{11}$. Per le donne era raro usare foto personali come loro avatar o nel loro profilo su QZone, e molte donne seguivano la prassi di "bloccare" il loro album QZone, di solito con domande del tipo "Come mi chiamo?", in modo da consentire l'accesso solo a chi le conosceva già personalmente. Nella Cina rurale la normatività online si esprime attraverso la reinvenzione delle relazioni basata sugli ideali tradizionali. Donne e uomini condividono pubblicamente materiali che rappresentano ciò che loro considerano 
come relazioni familiari tradizionali, con fotografie che ritraggono bambini e genitori, sposi felici, e memi con messaggi romantici. La piattaforma QQ è diventata uno spazio importante per esprimere questi valori familiari e romantici tradizionali, che considerano incarnare ideali modellati dal confucianesimo.

Anche nel sito italiano la visibilità limitata delle figure di donne negli spazi pubblici corrisponde a una assenza di visibilità sui social media. È estremamente insolito per le donne sposate postare foto di se stesse su Facebook, che limitano alle occasioni speciali, come feste di compleanno, riunioni familiari, o eventi specifici con amiche. L'assenza di fotografie che rappresentano il loro corpo è bilanciata dall'abbondanza di immagini di oggetti domestici, memi, fotografie artistiche o foto dei loro figli. In questo caso, le trasformazioni nel corso della loro vita riproducono il modo in cui cambia la loro visibilità negli spazi offline della città. Dalle donne sposate, specie dopo che sono diventate madri, ci si aspetta che mutino il modo in cui appaiono negli spazi pubblici e che esaltino i loro ruoli di mogli e madri. Di conseguenza sui social media non mostrano apertamente immagini di se stesse, che potrebbero essere interpretate come segni di flirt.

$\mathrm{Al}$ confronto, uomini adulti e amicizia fra uomini sono più visibili sui social media pubblici. Gli uomini adulti inoltre condividono tipici contenuti convenzionalmente associati alla maschilità, come la politica, le moto di grossa cilindrata e lo sport. Sono anche relativamente creativi nel postare. Per esempio, possono pubblicare foto per creare poster o specie di memi personalizzati, oppure usare una gamma più diversificata di aggiornamenti di status. Al contrario delle donne, gli uomini sono inclini all'auto-ironia online, per esempio, usando software gratuiti online per creare avatar di se stessi o brevi storie in cui si prendono gioco di situazioni specifiche della loro vita. Un altro esempio del modo in cui le norme di genere dominanti vengono riprodotte e rafforzate negli spazi più pubblici dei social media arriva dal nostro sito nel sud dell'India. Qui il genere emerge come un elemento dei tradizionali ruoli familiari, come il comportamento della sposa novella o della suocera. Le famiglie si impegnano collettivamente nei profili Facebook dei singoli membri della famiglia, in modo che l'effetto complessivo sia quello di dare all'esterno un'immagine di famiglia rispettabile e idealizzata. L'obiettivo è evitare qualsiasi apparenza di disputa o di disarmonia familiare, e rispettare online i ruoli familiari di genere è importante per questa rappresentazione. Le piattaforme di social media pubblici come 
Facebook, Instagram o QQ offrono uno spazio per la costruzione consapevole della propria immagine, mettendo in scena il sé en $^{12}$, come nel caso del sud dell'India, la famiglia. Mentre certi post possono essere rivolti a individui o gruppi specifici, i social media pubblici creano anche la sensazione di un pubblico più generale o di un'audience generica immaginata.

Il sito nel nord del Cile conferma ancora una volta l'osservazione che i social media pubblici hanno rafforzato le norme di genere; qui, entrambi donne e uomini, si comportano secondo le aspettative sociali, presentando una rappresentazione limitata di modelli di genere. Haynes scrive che spesso la differenziazione fra il lavoro degli uomini e il lavoro delle donne rafforza le norme di genere: gli uomini prevalentemente lavorano in miniera, nell'edilizia urbana e portuale, mentre le donne gestiscono la casa o lavorano nel servizio ai clienti o nelle professioni di cura. Sui social media l'orgoglio maschile nel lavoro è molto spesso espresso attraverso la rappresentazione del lavoro come un sacrificio di tempo necessario per mantenere la famiglia. Al contrario, il frame del lavoro femminile riguarda la cura della famiglia, un ruolo che può apparire come la naturalizzazione della differenza di genere. Questo tipo di rappresentazione a volte sopprime la variabilità individuale e le categorie di genere appaiono di gran lunga più nette di quanto non siano nel mondo offline, dove tantissime donne mettono in moto le loro auto e molti uomini sono tranquillamente dediti a cucinare torte.

Un pubblico generico corrisponde anche all'audience immaginata dei post visivi che spesso prevalgono sui social media. Il vantaggio di studiare le norme di genere mediante i social media emerge forse meglio dall'altro nostro volume comparativo, Visualising Facebook ${ }^{13}$. Ciò anche perché quel libro consta in gran parte di post visivi, mettendo i lettori letteralmente in grado di vedere come appaiono le norme di genere in differenti popolazioni nei siti in Inghilterra e a Trinidad. I ricercatori in ciascun sito hanno guardato migliaia di immagini fotografiche postate online. Ne risulta che le convenzioni e i tipi principali cui si ricorre nella rappresentazione di forme normative come il genere diventano evidenti dopo un po' di tempo e sono chiaramente supportate. Il punto è che, in quanto accademici, possiamo ormai letteralmente percepire le norme culturali come la costante ripetizione di immagini, e così anche le persone di cui stiamo guardano le immagini.

In particolare possiamo "vedere" come funziona la riproduzione delle identità di genere, spesso attraverso una serie di associazioni in contrasto. Per esempio, il materiale inglese mostra un'associazione molto comune fra 
maschi e birra. Esaminando i post online questa associazione compare centinaia di volte. Di contro, un certo numero di post istituisce un legame altrettanto forte fra donne e vino. Non c'è mai nessuna indicazione sui diversi tipi di vino, o su eventuali interessi o competenze femminili circa tali differenze. Emerge un'unica generica categoria "vino", a partire da cui si costruiscono molti memi divertenti incentrati sullo scarto tra il lavoro domestico che ci si aspetta che le donne facciano mostrando interesse per quella specifica attività, e il fatto di stare sedute con soddisfazione con un bicchiere di vino in mano. I memi possono semplicemente esprimere la passione per il vino come in "Al mio club del libro si leggono solo etichette di vini", o "Non sto bevendo di nuovo... Oh guarda... vino". Il vino ha dato alle donne qualcosa da applicare in modo simmetrico all'uso maschile della birra. Offline considereremmo l'abbigliamento come il mezzo attraverso cui il genere si confa alle norme, ma online dove, come in questo caso, il bere è diventato una sorta di vestito, ci sono spazi più ampi per potenziali distinzioni di genere che si possono rendere significative.

Le corrispondenti differenze nei post visivi a Trinidad di solito si basano sul genere in quanto dimensione fisica: immagini femminili collegate al glamour, alla sessualità e alla carne, maschilità legata all'abbigliamento e agli accessori. Molti uomini appaiono con quello che è chiamato "metallo": per esempio, pesanti catene d'oro, anelli o bracciali che si suppone indichino ricchezza e anche forza. A Trinidad le norme di genere si intrecciano anche con la classe e con l'etnicità. Per esempio, uomini giovani afro-trinidadiani tendono a sottoscrivere o ad assumere l'immagine "gangsta" che deriva dall'hip-hop statunitense, postando immagini di se stessi con occhiali da sole, jeans a gamba larga, vestiti di marca e gioielli vistosi; gli uomini indo-trinidadiani, invece, su Facebook sottolineano la loro immagine professionale o la loro vocazione. Le donne di classe media solitamente postano su argomenti quali stile di vita e consumo; a qualsiasi età, nelle loro immagini, tendono a evidenziare gli elementi attraenti e, in ogni caso, non posterebbero mai un'immagine in cui non apparissero attraenti. Ancora una volta, questo è in forte contrasto con l'uso inglese. Non ci sono segni di un qualsiasi cambiamento o trasformazione negli usi femminili e in quelli maschili di queste associazioni. Semplicemente, si rende molto più visibile la dimensione materiale più ampia legata all'essere maschi o femmine. Se c'è un elemento addizionale, questo riguarda la capacità di prendersi gioco di tali associazioni mediante altri elementi visivi come i memi. È molto fre- 
quente che le donne si lamentino del modo in cui un'altra donna ha scelto di presentarsi online e lo denigrino, ma questo sembra essere esattamente come accade offline.

Per concludere questa parte, abbiamo trovato che in molti dei nostri siti si pone un'associazione fra visibilità delle piattaforme di social media più pubblici e conservatorismo delle norme di genere rappresentate on line, che spesso supera quello offline. In un'ottica di genere, social media pubblici come Facebook potrebbero essere descritti quasi come iper-conservatori. Questo sembra un chiaro riflesso del grado di controllo assoluto al quale le immagini sui social media pubblici sono soggette. Questi pertanto sono diventati luoghi in cui gli individui vogliono dimostrare la loro adesione alle norme culturali, e trovarne conferma.

\section{Discontinuità}

Ci occupiamo ora dei modi in cui i social media hanno portato a una rottura delle norme e delle pratiche di genere offline. Nei nostri siti, lo scombussolamento delle norme di genere esistenti provocato dai social varia molto, e gli elementi in comune fra i diversi siti sono pochi. Inoltre, il focus passa da azioni dirette alla costruzione del sé da mostrare su spazi online semi pubblici e pubblici alle implicazioni dei social media in quanto siti in cui le persone interagiscono, a volte in situazioni online più chiuse. Nella Turchia sudorientale l'impatto dei social media sulla trasformazione delle relazioni di genere forse è più significativo che quello sul conformismo. I social media hanno ampliato moltissimo le occasioni delle donne di relazioni sociali laddove queste nel mondo offline sono ridotte. A Mardin la presenza di donne in spazi pubblici è già aumentata negli ultimi dieci anni, con maggiore accesso femminile all'istruzione e maggiori opportunità di lavoro fuori casa. Tuttavia, molte giovani donne esprimono il desiderio di maggiori opportunità di estendere le loro reti sociali, lamentando non solo la mancanza di eventi sociali, ma anche il controllo che genitori o mariti esercitano su di loro. Dato che, di norma, hanno un accesso limitato agli spazi pubblici come bar, ristoranti e strade, le giovani usano i social media per mantenere relazioni sociali e ampliarle fuori dal controllo familiare. Nel caso di donne con un retroterra più conservatore e religioso, di fatto confinate in casa perché i padri o i mariti non permettono loro di studiare, lavorare o incontrare 
amici, i social media diventano un mezzo di straordinaria importanza per stare in contatto con il mondo esterno. Anche se fisicamente a casa, possono lo stesso stare in contatto con gli amici spesso ricorrendo a un account falso che ne garantisce l'anonimato.

Così come si sono creati nuovi spazi per l'autonomia delle donne, sono emerse anche nuove possibilità per gli uomini di controllare le loro parenti, per flirtare e molestare altre donne e per ingannare le mogli. Grazie alla facilità con cui estranei e amici di sesso opposto possono dialogare, i social media sono diventati anche il luogo principale del corteggiamento e dove si vivono le relazioni amorose. Mentre le possibilità offline restano molto limitate, per giovani non sposati è adesso molto comune trovare una storia d'amore mediante la messaggistica privata di WhatsApp, Facebook, Viber, Tango o via SMS.

Anche nell'Italia del sud i social media hanno espanso le occasioni di comunicazione per le donne. Nonostante siano meno visibili online, le donne tendono a usare i social media come WhatsApp e i commenti su Facebook con maggiore intensità degli uomini, specialmente nelle famiglie più a basso reddito. Ancora una volta, sono le donne con opportunità più limitate offline per le ragioni di rispettabilità, a fare maggiormente uso dei social media in quanto importante strumento di comunicazione con un esteso network di altre persone. Gli uomini hanno più occasioni di conversare $\mathrm{e}$ di partecipare a eventi sociali offline, spesso incontri nei bar, nelle strade e in piazza. Di contro, le donne raramente si fermano a parlare in strada per timore di sembrare indolenti. Come abbiamo visto nella parte precedente, questa comunicazione così estesa viene bilanciata dal modo in cui queste donne sviluppano in parallelo strategie a difesa della loro rispettabilità come madri e mogli.

Nella Cine rurale, come nella Turchia sudorientale, i social media hanno aperto nuove opportunità d'interazione fra donne e uomini. Nella cultura cinese c'è stata una lunga tradizione di "interiorità femminile", con le donne storicamente confinate entro la sfera domestica. Benché gli ultimi decenni abbiano portato grandi aumenti di libertà e di partecipazione nel lavoro per le donne, nondimeno esse restano fortemente associate alla sfera domestica; la mobilità ha a che fare con gli uomini. Per fare un esempio estremo, è tipico che le donne siano confinate in casa durante il periodo di "sitting the month" immediatamente successivo alla nascita di un figlio. Di fatto oggi molte donne usano i social media per mantenere regolarmente i contatti con 
gli amici condividendo online foto del bambino.

Nello stesso tempo, i social media hanno anche allargato i network sociali degli uomini: il loro accesso più facile alle auto, per esempio, significa che possono viaggiare con molta più facilità delle donne. Molti uomini viaggiano spesso e alcuni usano servizi per la ricerca localizzata tipo Momo o "People Close by" di WeChat per parlare con estranei, di solito di sesso opposto. Alcuni uomini hanno detto a McDonald che tramite questo canale avevano organizzato tresche con donne residenti in grandi aree urbane; e anche se probabilmente si trattava di numeri piccoli, era stato sufficiente per provocare nella popolazione locale un timore diffuso sull'impatto dei social media sul matrimonio. Le attività maschili sui social media erano in parte legittimate anche dall'aspettativa della maggiore "espansività sociale" degli uomini rispetto alle donne, e dal fatto che essi coltivavano connessioni sociali con altri uomini. In questo senso, gli uomini tendono a essere utenti più pesanti delle applicazioni dei social, il che aiuta a ottenere "coperture" per attività vietate.

In India del sud la situazione è per alcuni aspetti simile. È relativamente comune trovare giovani donne di casta alta e giovani uomini che usano i social media per flirtare, nonostante le significative differenze di classe sociale e di casta. Il principale effetto dei social media è stato consentire alle persone di incontrarsi al di là dei confini delle loro reti sociali tradizionali, espandendo i contatti oltre il posto di lavoro e le istituzioni formative. Venkatraman mostra come, nel suo sito, giovani uomini facciano amicizia con donne di altri stati indiani, o anche di paesi stranieri come il Brasile o gli Stati Uniti.

L'idea generale è che quando gli uomini usano Facebook potrebbero fare amicizia con donne di tutto il mondo, con le quali potrebbero arrivare a flirtare online o potenzialmente arrivare ad avere relazioni d'amore offline. Per riuscirci, in genere mentono sul loro status sociale, affermando di frequentare una prestigiosa università o di essere impiegati presso una nota azienda, sperando che queste affermazioni possano accrescere il loro appeal. Nel sito indiano, come in quello della Turchia sudorientale e della Cina rurale, questo desiderio di provare nuove relazioni intime e di amicizia spinge sia le donne sia gli uomini all'interno di un ampio network di persone. In questi tre diversi siti, i social media hanno aperto canali di comunicazione privati nuovi, cambiando la nozione di amore e ridefinendo le nozioni locali di maschile e femminile. Le reti sociali delle donne si sono allargate e in alcuni casi quelle degli uomini ancora di più. 
Nel sito in Brasile, i social media sembrano aver contribuito a una maggiore uguaglianza di genere in riferimento a numerosi ambiti della vita sociale. In conseguenza dell'espansione della tecnologia della comunicazione, della crescita dell'industria del turismo e dell'espansione della Chiesa Protestante, le donne hanno ora più accesso alle opportunità di lavoro e più visibilità pubblica. Spyer suggerisce che i social media hanno contribuito a un movimento generale verso la visibilità di nuove identità femminili e maschili alternative, che riguardano nello stesso tempo sia il mondo online sia quello offline. I profili Facebook, per esempio, si usano per condividere immagini che mostrano il proprio orgoglio per l'autonomia guadagnata grazie a un lavoro regolare. Il lavoro è diventato l'alternativa alla subordinazione nei confronti all'uomo, al punto che gli imprenditori dell'area preferiscono assumere donne, considerate più produttive e responsabili. Le giovani donne nel complesso non considerano la famiglia come il solo o il principale obiettivo nella loro vita; avere una carriera e guadagnare denaro è ugualmente importante.

Una rottura significativa con i modelli normativi di femminilità e maschilità si è verificata nella Cina industriale. Mentre nella Cina rurale i social media più pubblici vengono usati per rafforzare le nozioni normative di amore e famiglia, nella Cina industriale, tra gli operai, sia donne sia uomini si mettono in scena pubblicamente in modi non convenzionali. Nella Cina industriale i social media sono uno spazio dove le persone possono sperimentare ciò che non possono nelle loro vite offline, e funzionano in parte come uno scudo contro i vincoli che pesano sulle persone offline. Nel sito della Cina industriale, le costrizioni sociali che governano il comportamento pubblico sono meno forti e rigide che altrove. Qui i social media permettono alle persone di mettere un mondo di fantasia desiderato. Gli uomini appaiono mostrare una maggiore variazione fra la loro vita online e offline rispetto alle donne. Nella loro quotidianità devono rispettare norme di maschilità chiare che non includono romanticherie, dolcezza e sensibilità; devono essere duri emotivamente e decisi, e nei luoghi offline della socialità, come a tavola durante la cena, parlano di politica e raccontano barzellette sconce. All'opposto, sui social media si sentono liberi di esprimere il loro lato romantico e condividono gli stessi post sull'amore delle donne. Rappresentano se stessi come giovani donne, e esprimono ideali nettamente amaschili. Facendo così, si oppongono alle aspettative sociali, anche se avrebbero paura di perdere la faccia se esprimessero offline tali sentimenti. 
Parlando delle donne, Wang descrive come "sajiao" ("agire come un bambino viziato") una strategia comune usata dalle donne giovani per ottenere l'affetto degli uomini nelle situazioni offline. Oltretutto, è socialmente accettabile che le donne si rappresentino come dipendenti e vulnerabili, enfatizzando la loro debolezza e il bisogno di aiuto per ricevere attenzione, cura o favori da parte di altri. Ciò che è cambiato è che online postare sajiao visivi è popolare sia per i lavoratori migranti maschi sia per le femmine. Gli operai cinesi maschi tendenzialmente vivono in condizioni molto dure; in questo contesto i social media diventano uno spazio molto importante dove sono in grado di ritagliare vite alternative entro un mondo più desiderabile. Di contro, fra il mondo online e quello offline per le donne c'è maggiore coerenza, per quanto essere pubblicamente rispettosi della morale e rispettabili resta una priorità in entrambi gli ambiti.

\section{Sessualità non-eteronormative}

Non sorprende che i social media abbiano influenzato anche la popolazione LGBT. Nelle aree urbane più cosmopolite i media digitali sono stati usati dagli attivisti LGBT per impegnarsi nella politica organizzata, facendo sì che l'identità gay acquistasse maggiore visibilità. Nel sito in Brasile, i social media hanno contribuito a un processo di generale aumento di visibilità della popolazione LGBT, parte di un più ampio processo favorito dai social che ha reso le peculiarità individuali e collettive più evidenti. Nel contesto di una cittadina come Mardin, invece, i social media hanno semplicemente facilitato la capacità di incontrarsi. In posti come la Turchia sud orientale dove essere dichiaratamente gay è socialmente inaccettabile, i social media hanno creato più occasioni tra gli uomini di interazioni segrete. Le identità gay non sono divenute più visibili e pubbliche, ma gli incontri omosessuali possono ora essere organizzati in modo riservato con più facilità.

Nel Cile del nord, dove le pratiche sessuali non normative sono in qualche modo più accettate, i social media a volte si usano per rivelare queste identità, per esempio su Instagram con selfie etichettati come \#instagay o \#instalesbian. Grindr è una app diffusa anche fra uomini in cerca di un partner dello stesso sesso (sia per incontri romantici sia solo sessuali), benché lo sia per lo più fra gli uomini più ricchi nella città più grande di Iquique. La presenza di gay e lesbiche sui social media pare accettata finché essi si mettono in scena 
secondo modalità di genere normate, per esempio restando in stretto contatto con la famiglia d'origine e facendo lavori considerati adatti al loro genere; in questi casi raccontano di aver subito poche discriminazioni. Coloro che invece agiscono fuori dalle norme di genere subiscono disapprovazione. Pertanto, gli individui che si rappresentano come drag costruiscono profili falsi sui social media a tale scopo, "per non far preoccupare la mamma", come racconta uno di loro.

Ovviamente, come è evidente negli esempi della Turchia sudorientale e del Cile del nord, la visibilità dei post influenza il tipo di informazioni che possono essere rivelate. Così, nel sito inglese, un gay, un uomo di mezza età posta continuamente immagini esplicitamente sessuali e a volte deliberatamente oltraggiose sul suo Facebook; e, ancora, una giovane lesbica non si riferisce mai esplicitamente alla sfera sessuale, ma la sua relazione con le donne può essere dedotta dalle sue fotografie. In questi casi, la disinvoltura con cui gli uomini rappresentano pubblicamente la sessualità e la relativamente meno esplicita rappresentazione delle donne continuano a seguire le norme di genere. Non solo ciò illustra l'ambito di visibilità rispetto a cui persone diverse sono a loro agio, ma mostra anche i modi in cui i ruoli di genere più tradizionali continuano a influenzare l'uso dei social media di uomini e di donne, anche quando essi si identificano come LGBT ${ }^{14}$.

\section{Conclusioni}

In questo capitolo abbiamo visto quando e dove i social media hanno rafforzato, distrutto o solo modificato sistemi di relazioni di genere e norme di genere. Gli esempi dimostrano che internet, e le tecnologie digitali più in generale, non sono in se stessi innovativi o conservativi. Al contrario, essi esistono solo dentro pratiche sociali variabili. Se, come si sostiene in questo libro, il mondo ha cambiato i social media, allora ne è esempio il modo in nei social media si sono inscritte relazioni di genere diverse, e idee diverse di cosa significhi essere uomo o donna. Le aspettative culturali relative agli uomini e alle donne in Turchia e a Trinidad difficilmente potevano essere più diverse tra loro. A Trinidad, ci sono occasioni, come il periodo che precede il Carnevale, durante le quali le donne cercano di esibire quanta più carne possibile nell'ambito di una sessualità roboante, accanto alla iper-maschilità nello stile "gangsta" degli uomini. All'opposto, le donne religiose nel sudest 
della Turchia si preoccupano moltissimo di evitare la visibilità della carne, persino di un solo capello. In ciascun caso c'è una notevole variazione interna. Lo stesso sito includeva un'ampia gamma di preoccupazioni diverse riguardo alla rispettabilità, dal momento che entrambi i siti includono anche laici, individui etnicamente diversi e un ampio spettro di religiosità, per esempio, a Trinidad, il Pentecostalismo. In altri posti, come i nostri siti in Italia del sud o nella Cina rurale, le principali differenze emergono nel corso degli eventi della vita, con le donne che cambiano radicalmente le loro rappresentazioni online in seguito al matrimonio o alla maternità.

Non abbiamo tuttavia limitato la nostra analisi all'osservazione del modo in cui i social media riproducono le relazioni di genere. L'opportunità unica di comparare nove diversi siti ci ha permesso di identificare e sottolineare configurazioni comuni e di fare generalizzazioni antropologiche. Abbiamo scoperto che in quasi tutti i nostri siti (eccetto la Cina industriale e in una certa misura il Brasile), i social media più pubblici hanno rafforzato la normatività di genere e sono spesso più tradizionalisti della socialità di genere offline. Al contrario, mentre nei contesti offline le donne e gli uomini hanno la tendenza a mettere in scena pratiche e ruoli più vari, a volte incorporando aspetti sia maschili sia femminili, sui social media tendono ad aderire strettamente alle aspettative della società riguardo a come ci si dovrebbe comportare. Spazi social più pubblici, come Facebook o QZone, hanno creato spazi estremamente conservativi dove le donne e gli uomini si fanno particolarmente attenti agli ideali di genere. A questo riguardo le parti pubbliche dei social media emergono come l'antitesi dell'uso di internet precedente allo sviluppo di queste piattaforme, quando venne salutato come uno spazio di liberazione da parte delle teoriche del femminismo. Anche se "online" può riferirsi sia a internet di prima sia agli attuali spazi social, le conseguenze sul genere sono state davvero diverse.

$E$ ancora, dato che i social media sono una configurazione di forme diverse di socialità modulabile, essi includono anche gruppi più piccoli e più privati dove donne e uomini sono meno preoccupati di seguire i comportamenti appropriati. Ciò riguarda piattaforme come WhatsApp o altra messaggistica privata. Uomini e donne trovano inoltre il modo di interagire nell'anonimato. Sono proprio queste opportunità a creare nuove e inusuali relazioni sociali che hanno portato alla disgregazione delle relazioni di genere esistenti e dei ruoli di genere. Una delle conseguenze più significative dei social media potrebbe consistere nel fatto che le donne che vivono in condizioni di 


\section{Genere}

massima costrizione possono ora, per la prima volta, trovare modi per creare relazioni online personali con persone che prima non avrebbero mai potuto incontrare. Per questo motivo, in conclusione, è importante capire i modi in cui i social media possono simultaneamente essere sia più conservativi sia più liberatori rispetto alla vita offline. 



\section{Capitolo 9 \\ Disuguaglianza}

Come ci si potrebbe attendere, c'è un notevole interesse per la capacità di internet e dei social media di produrre cambiamenti sociali di grande scala. Eppure persiste il dubbio se l'accesso a internet e ai social media abbia migliorato la situazione critica delle popolazioni più svantaggiate del mondo o abbia invece esacerbato le disuguaglianze e si è lontani da una risoluzione. Come indicato nei precedenti capitoli, i social media hanno avuto un importante impatto sull'istruzione, sul lavoro e sulle relazioni di genere, tutte componenti preminenti di tale questione più ampia. Diversi dei nostri campi di ricerca rappresentano popolazioni a basso reddito e svantaggiate. Qui esaminiamo i modi in cui i social media possono avere un impatto sulle persone che non hanno facile accesso alle risorse digitali, e il modo in cui il loro uso potrebbe essere un modo per cambiare o, al contrario, per confermare la loro attuale posizione sociale.

Il numero di persone che usano la comunicazione digitale è aumentato sensibilmente dal lancio dell'accesso commerciale a internet alla metà degli anni Novanta del Novecento. E non si tratta solo dei ricchi, cosmopoliti e istruiti; la combinazione attuale di tecnologia mobile e social media ha creato un forte interesse fra varie popolazioni socialmente deprivate, inclusi popolazioni analfabete o semi-analfabete, lavoratori manuali migranti sottopagati e migranti, in posti come la Cina, l'India e il Brasile ${ }^{1}$.

Come negli altri capitoli di questo libro, qui saranno presentate le risultanze del nostro impegno etnografico di lunga durata presso nove differenti popolazioni. Vedremo che in ogni posto esiste disuguaglianza e si esprime in modi diversi, a seconda dei processi storici, delle politiche attuali e delle strutture sociali. Trarre comparazioni, per questo, non sempre è lineare. In ogni sito le disparità di guadagno e ricchezza, così come di status sociale, sono associate con altre forme di differenza sociale del tipo genere, età, istruzione, religione e disuguaglianza razziale. I gruppi in ogni campo di ricerca hanno una percezione della propria posizione sociale che è largamente relativa agli altri della stessa società, piuttosto che essere messa a fuoco sullo sfondo di una qualche scala astratta. 


\section{Che cos'è la disuguaglianza?}

Se asseriamo che la disuguaglianza esiste in varie forme, che cosa esattamente intendiamo quando ci chiediamo se i social media influenzino la disuguaglianza? Di certo una forma di disuguaglianza riguarda la distribuzione della ricchezza e la povertà. Spesso pensiamo alle persone più svantaggiate come a quelle che vivono negli slums, senza possibilità di lavoro e senza speranza in un futuro migliore. Eppure le disuguaglianze economiche sono spesso il risultato concomitante di più fenomeni, come il razzismo, l'assenza di rappresentanza politica e lo scarso accesso sia alle risorse materiali, come acqua potabile o elettricità, sia a quelle più astratte come l'istruzione. In alcuni dei nostri siti le persone non sono necessariamente ridotte in povertà, ma prive di potere politico. In altri, una frettolosa analisi dei loro beni, di cui potrebbero far parte televisori a schermo piatto e telefoni cellulari Samsung di ultima generazione ${ }^{2}$, nasconde il fatto che a volte essi non possono pagare l'elettricità. In altri casi, disuguaglianza significa semplicemente che certe parti della popolazione sono discriminate in termini di accesso alle risorse, sulla base di tratti come la razza o la religione . $^{3}$.

Bourdieu ha indicato tre diversi tipi di disuguaglianza corrispondenti a diversi tipi di "capitale". Il capitale economico, in genere relativo all'accesso al denaro. Il capitale sociale, che descrive le relazioni sociali e le reti istituzionalizzate di cui un individuo è parte. Il capitale culturale, che include conoscenze o capacità ottenute mediante l'istruzione, beni culturali, qualifiche. Ciascuno di questi tipi di capitale è influenzato dagli altri, e la attenzione primaria di Bourdieu riguarda il modo in cui sono utilizzati dai gruppi elitari per riprodurre i loro privilegi.

Connesso alla disuguaglianza è il concetto di mobilità sociale, che si riferisce alla capacità di un individuo o gruppo di migliorare la propria posizione sociale. Di nuovo, ciò può assumere molte forme, dal posto di lavoro migliore alle risorse formative così che i figli avranno prospettive future migliori. La mobilità sociale non significa solo avere più soldi, ma mostrarlo nel modo giusto; in sostanza mettersi in scena come parte di una particolare classe sociale. Ciò può significare acquistare i marchi "giusti", possedere i "giusti" apparecchi; mandare i figli alle scuole "giuste" o anche vestire in un modo che è conforme alle norme di quella classe sociale. Ciò che è "giusto" è

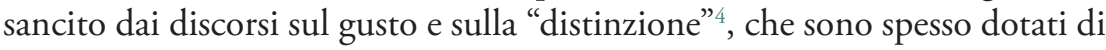
un valore morale ${ }^{5}$. Ciò spesso richiede volgere il capitale economico in capitale sociale e culturale o anche trovare i modi per acquisirlo in mancanza di 
capitale economico. La tecnologia mediatica è diventata uno dei principali modi in cui certe persone meno privilegiate possono farsi capaci di accedere a risorse, quali l'informazione, prima disponibili solo ai più privilegiati. Non sorprende dunque che internet possa essere situato fra gli strumenti di mobilità sociale.

Ne consegue che facilitare l'accesso ai new media si ritiene sia diventato basilare per lo sviluppo moderno e per aiutare le persone a trovare una "voce" ${ }^{6}$. Persone che non hanno accesso a internet sono prive di possibili risorse cui potrebbero accedere online. Senza internet sperimentano nuove e ulteriori barriere al miglioramento del loro accesso ai capitali economico, sociale e culturale, mentre il resto della società è in grado di ottenere risorse maggiori grazie all'accesso alle nuove tecnologie. Questa mancanza di accesso perciò emerge come una forza capace di esacerbare e ampliare precedenti forme di disuguaglianza. L'accesso a internet però non si traduce automaticamente in maggiore accesso alle informazioni e alle risorse ${ }^{7}$. I nostri campi di ricerca hanno infatti mostrato che i discorsi precedenti sulle differenze e sulle distinzioni continuano a influenzare, in grande misura, i modi particolari in cui le persone usano internet e i social media, spesso riflesso della classe sociale. Per di più, è del tutto possibile che la diffusione straordinaria di smartphone e social media rappresenti in sé e per sé una forma di maggiore uguaglianza, ma senza che ciò abbia necessariamente una qualsiasi ricaduta sulla disuguaglianza offline.

\section{Approcci a social media e disuguaglianza: il positivo, il negativo, il "grounded"8}

La letteratura rilevante può essere grosso modo divisa in due campi quasi del tutto opposti. Il primo sostiene che i social media sono destinati a introdurre maggiori disuguaglianze nella società perché concentrano le risorse educative e relazionali fra coloro che sono già privilegiati. In alternativa, l'approccio "tecno-utopico" vede i social come una panacea per i problemi della disuguaglianza, poiché offrono alle persone svantaggiate accesso a risorse più grandi mediante internet.

La letteratura appartenente alla categoria del "digital divide" spesso sostiene che le nuove ICT aumentano preesistenti disuguaglianze nelle società: gli individui più poveri sono esclusi, mentre i più ricchi usufruiscono di accessi 
migliori. I primi studi, focalizzati sull'accesso a internet e non tanto ai social media, furono realizzati in gran parte all'interno di paesi sviluppati e posero l'enfasi sul fatto che, benché la grande maggioranza della popolazione avesse accesso a internet, una minoranza rilevante o non ne aveva affatto o aveva connessioni sotto la media. Spesso i vincoli che impedivano alle persone di beneficiare della comunicazione online erano imposti da fattori come l'età, il reddito familiare, il livello d'istruzione, il livello di conoscenza dell'inglese, la disabilità, la residenza rurale o urbana?.

Via via che l'accesso a internet è migliorato e i social media e altre risorse online sono divenuti sempre più disponibili, gli studiosi hanno indicato l'emergere di altri tipi di "spartiacque". Molto dipende dalle differenti forme di accesso, dalle specificità del contesto locale, che esercitano un'influenza su come le persone usano queste tecnologie ${ }^{10}$. È stato proposto che il "network divide" ha un impatto più forte del "digital divide", e che la distinzione chiave oggi riguarda la capacità delle persone di acquisire le competenze necessarie per coltivare con successo i loro social network online ${ }^{11}$. Questa trasformazione è stata influenzata da un accento sempre crescente sulle "alfabetizzazioni digitali” da parte di alcuni studiosi, che enfatizzano che padroneggiare l'uso di questi network è divenuto tanto importante quanto l'essere semplicemente capaci di accedervi (come discusso nel Capitolo 5) ${ }^{12}$. Infine, ci sono approcci che guardano di più alle disuguaglianze sistemiche globali. Anche se gli individui hanno sia accesso sia capacità, restano enormi squilibri fra la quantità di contenuto disponibile in lingue diverse o prodotto in aree del mondo come America Latina, Africa e India ${ }^{13}$.

Nonostante queste questioni sulla disuguaglianza d'accesso sopra menzionata, i discorsi "tecno-utopici" affermano che internet rappresenti egualitarismo, libertà di parola e democrazia ${ }^{14}$. Questi lavori descrivono i social come uno strumento che può essere usato per consolidare il potere collettivo contro istituzioni potenti, spesso rappresentate attraverso la polarità dell'"individuo contro il governo" o del "consumatore contro le corporazioni aziendali”. Questi discorsi suggeriscono, pertanto, che il networking sociale agisca come una forma di empowerment, promuovendo l'attivismo civile che sfida i sistemi che producono disuguaglianza ${ }^{15}$.

Come dimostrato nel capitolo precedente sul genere, i primi osservatori di internet si domandavano se le comunità virtuali online che erano emerse durante gli anni Novanta avrebbero favorito l'uguaglianza lasciando le persone libere di creare identità immaginarie online indipendenti dai loro 
corpi. Questo tipo di concetto ha implicazioni anche per le disuguaglianze basate su altri aspetti dell'identità, per esempio l'età, la razza, la ricchezza o la classe ${ }^{16}$. Una questione complementare è se, dato il potenziale dell'anonimato online, le relazioni sociali potessero esistere a prescindere dalle differenze incorporate o da altre circostanze "offline" e se, invece, si potesse apprezzare il dominio online come un nuovo spazio indipendente in cui la mente potesse godere della libertà da vincoli precedenti ${ }^{17}$. Tale questione è una componente chiave del capitolo precedente sul genere.

Meno evidente in entrambe queste categorie della letteratura, e di più grande rilevanza per il nostro progetto, è stato il modo in cui la disuguaglianza in se stessa può significare cose diverse per persone diverse. Un numero crescente di studiosi ha preso le distanze da lavori che presumono che i social media debbano avere un effetto positivo o negativo sulla disuguaglianza, invitando invece ad approcci che considerano il complesso, sfumato e spesso contraddittorio spettro di effetti che i social media hanno sulla "realtà caotica" delle esistenze delle persone ${ }^{18}$. Approcci etnografici più fondati riguardanti l'uso di internet da parte dei teenager negli USA suggeriscono che molti dei proble$\mathrm{mi}$ che i giovani affrontano online restano radicati in disuguaglianze sociali e razziali di lunga data ${ }^{19}$. Per esempio, uno studio che traccia il passaggio di teenager americani istruiti, bianchi, di classe media da MySpace a Facebook metteva in relazione la percezione che Facebook fosse in grado di dare maggiore sicurezza rispetto a MySpace con il "White flight" (un fenomeno per cui bianchi ricchi si spostano in periferia, lontano dalla popolazione non bianca residente nei centri urbani) ${ }^{20}$. Un altro studio condotto negli USA guarda a come l'uso dei social media possa accentuare le differenze di classe, dal momento che genitori svantaggiati esercitano un maggior controllo sull'uso dei social media da parte dei loro figli per equilibrare i rischi che queste persone giovani corrono vivendo in quartieri meno abbienti ${ }^{21}$. Il nostro progetto ha preso altre vie ${ }^{22}$ per estendere lo scopo di queste inchieste e in particolare per comparare la disuguaglianza trovata all'interno di ciascuno dei nostri siti con quella trovata tra i diversi siti tra loro.

\section{La diversità delle differenze}

Nei nostri campi di ricerca in Brasile e nella Cina rurale molti individui hanno elevate aspirazioni di mobilità sociale collegata all'istruzione. In par- 
ticolare, nella Cina rurale l'istruzione è vista come la chiave per la futura mobilità sociale. Eppure il nostro lavoro ci porta a essere anche cauti nel generalizzare questa osservazione comune sull'istruzione in Cina, poiché dal nostro sito di ricerca nella Cina industriale risulta evidente che la migrazione degli stessi lavoratori rurali nelle fabbriche ha creato una classe di centinaia di milioni di individui che probabilmente ora vedono l'istruzione come una cosa di poco valore, riconoscendo che sono destinati a entrare in fabbrica come forza lavoro sin da giovani. I social media sono tuttavia usati per condividere suggerimenti di auto-aiuto su QQ, in particolare consigli legati al successo finanziario, molto di frequente condivisi. In generale, i social media erano visti come un posto per rafforzare la cooperazione, per condividere informazioni su opportunità di lavoro o di formazione non tradizionale.

In Brasile i lavoratori manuali sottopagati aspirano all'istruzione come fondamento della mobilità sociale, ma l'effettiva qualità dell'istruzione locale disponibile in città era generalmente molto scarsa. Queste popolazioni di giovani a basso reddito usano i social media come una valida risorsa educativa alternativa. I video d'istruzione su YuoTube che insegnavano capacità lavorative, come Microsoft Word, erano popolari e spesso molto efficaci come risorsa per i giovani che speravano di trovare un impiego che avrebbe consentito loro di ottenere l'agognata mobilità sociale. Anche se non è il principale oggetto della nostra inchiesta, per molti dei nostri siti sarebbe stato difficile esagerare l'importanza crescente di YouTube, in particolare, come una modalità di istruzione informale.

In Brasile e in entrambi i nostri siti cinesi la capacità di accedere a queste risorse dava anche alle persone un senso di auto-stima, come se questa tecnologia evocasse la sensazione di muoversi da uno stile di vita "arretrato" verso la "modernità". In tutti e tre i siti gli informatori vedevano il campo del digitale come in grado di offrire una certa emancipazione, non tanto perché potevano avere lo stesso smartphone delle persone delle classi più ricche, ma per il fatto di avere un certo controllo sulla loro auto-presentazione. Tale controllo permetteva loro di costruirsi un'apparenza che si avvicinava di più al modo in cui ora sentivano di essere.

Le opportunità di autopresentazione però non sempre sono considerate vantaggiose. Nel sito italiano, dove la disoccupazione in tutti i gruppi è elevata, abbiamo visto (nel Capitolo 6) come i giovani laureati in prestigiose università usano i social media per dimostrare capacità professionali che possono agevolarli a ottenere un lavoro. Per i giovani di provenienza meno 
abbiente, tuttavia, i social media possono spesso presentare pressioni non gradite a costruirsi una immagine pubblica di se stessi. Questi giovani spesso frequentano scuole incentrate su capacità professionali quali, per esempio, quelle richieste per il mestiere di idraulico, di meccanico o di segretaria, e sono spinti a iniziare a lavorare prima possibile per contribuire al reddito familiare. Per loro, stare sui social media può essere avvertito come un peso legato a un obbligo sociale - per esempio come quando si va a un matrimonio chic e ci si deve vestire in modo appropriato - rendendoli così più consapevoli della loro bassa posizione sociale. Qui sono i genitori a spingere i figli a creare un account su Facebook, perché si preoccupano che l'assenza possa rendere esplicita la loro posizione di disuguaglianza.

Per questi italiani svantaggiati, dunque, stare sui social media spesso è un obbligo che preferirebbero non avere. Già ora, quindi, possiamo scorgere delle differenze molto nette nella relazione basilare tra social media e ambizione alla mobilità sociale.

\section{Rendere visibile la mobilità sociale}

Il controllo sulla presentazione, importante per gli operai brasiliani e cinesi, riflette la percezione che, nella vita moderna, chi si può essere dipende sempre di più da chi si appare essere. Per questo occorre una buona conoscenza degli atteggiamenti prevalenti riguardo al buon gusto, il che ci riporta alle precedenti considerazioni su come le persone cercano di convertire il capitale economico in capitale sociale e culturale. In Brasile, ottenere visibilità sociale grazie a una ricchezza materiale recentemente accresciuta e ad altri segni di realizzazione permetteva a persone di gruppi svantaggiati di rivendicare l'ingresso in nuove comunità. Ciò implica che i social media hanno spostato lo status sociale ancora di più in direzione della visibilità; oggi semplicemente è più facile e effettivamente più comune vedere persone, almeno online.

Il matrimonio di Sandra è un esempio di questo processo. Sandra, una brasiliana afro-discendente, venne data dai suoi genitori a lavorare come domestica quando aveva cinque anni. Questa pratica di "dar os filhos" 23 ("dare i propri figli") era comune fra le famiglie povere della regione, specie quelle di discendenza africana ${ }^{24}$. Così facendo i genitori si assicuravano che i figli avrebbero avuto cibo, vestiti e a volte sarebbero anche stati mandati 
a scuola mentre imparavano un mestiere, le ragazze quello di domestiche. Sfortunatamente, come era del resto comune in tali circostanze, Sandra e le sue sorelle furono esposte ad abusi fisici, emotivi e sessuali ${ }^{25}$. Sandra tuttavia considera positivamente un aspetto di quell'esperienza: l'essere entrata nella Cristianità evangelica.

La Cristianità le offrì un incentivo per imparare a leggere, per poter capire la Bibbia. In seguito, tramite le relazioni della chiesa trovò un lavoro come commessa part-time nella locale libreria cristiana del paesino. Questo lavoro informale paga la metà del salario minimo e non include benefit, ma Sandra può usare il computer del negozio durante le ore di riposo per giocare online e guardare film cristiani su YouTube. Lo scopo principale di Sandra a questo punto, considerando che ritiene di aver fatto la sua parte di lavoro duro nella vita, non è aumentare il suo capitale economico; desidera invece una forma di capitale culturale che abbia valore all'interno della sua specifica comunità di chiesa.

Quando Sandra sta progettando il suo matrimonio, lo vede come un modo importante per ottenere la piena appartenenza alla comunità evangelica. I Cristiani evangelici nel nostro sito in Brasile spesso appartengono alle famiglie locali più prospere. Grazie all'aiuto finanziario di amici e familiari, la sua cerimonia avrà decorazioni uguali a quelle viste nei programmi sui matrimoni in televisione: fiori, frutta, abito colorato e illuminazione di lusso, un vero e proprio abito nuziale e una festa per più di 300 invitati. Grazie a questa pompa, lei rappresentava se stessa come una persona che aveva pienamente compiuto un processo di mobilità sociale e come parte della comunità evangelica.

La sua famiglia, inoltre, affermò la propria forma di mobilità sociale scegliendo molte foto ufficiali delle nozze per mostrarle sull'account Facebook della sorella. Questo per una settimana diede l'opportunità a tutti gli invitati di trovarsi nelle immagini e di dire chiaramente quanto l'evento fosse stato meraviglioso. In sostanza, sia Sandra sia i suoi invitati poterono accedere a una forma di capitale culturale attraverso l'organizzazione, la partecipazione e infine il ricordo e la rappresentazione dell'evento tramite le fotografie sui social media. Proprio come i giovani nel sito brasiliano preferirebbero scattare un selfie in palestra o in piscina piuttosto che contro un semplice muro di mattoni, Sandra e i suoi ospiti sapevano che ritrarre se stessi come socialmente mobili era importante proprio come essere realmente in grado di organizzare raffinate decorazioni di nozze o essere socio in una palestra. 
Ciò per via del modo in cui diverse forme di capitale, come le risorse economiche, il gusto e i legami sociali, si coniugano, conferendo un'apparenza naturale al fatto che alcune persone hanno più privilegi di altre.

Dall'esempio di Sandra si può ricavare una lezione chiave, ossia che non possiamo dare per scontato che l'enfasi sulla visibilità sia da interpretare come una nuova forma di superficialità che arriva con i social media e il loro focus sull'apparenza. In questo caso la forza trainante è il principio fondamentale dei Cristiani Protestanti, che secoli prima dei social media sostenevano che solo attraverso l'apparenza un individuo poteva stabilire se stava fra i "salvati", aspirazione primaria per questo ramo della Cristianità ${ }^{26}$. Analoghi principi stavano anche dietro la spinta verso l'ascesa sociale attraverso il duro lavoro e la ricchezza. Zuckerberg può aver fornito i mezzi, ma Calvino concepì la causa.

\section{I limiti all'impatto dei social media sulla mobilità}

Sebbene in Brasile la rappresentazione visiva della mobilità in ascesa sia importante, la mera evidenza fotografica di beni considerati di buon gusto non garantisce l'accesso alle classi elitarie in tutti i contesti. Gli antropologi si imbattono in molte strategie per la mobilità sociale che falliscono, mentre le differenze di classe sono riprodotte mediante le azioni quotidiane, spesso inconsapevolmente ${ }^{27}$. Questi stessi discorsi quotidiani sul gusto sono usati per mantenere la disuguaglianza fra gruppi con livelli di ricchezza simili: certi gusti sono denigrati in quanto volgari, kitsch o poco sofisticati ${ }^{28}$ nelle critiche spesso associate alla razza, alla religione, alla regione, alla opposizione urbano / rurale e anche al senso di "vecchio" vs "nuovo" rispetto alle identità di classe (per esempio, la nuova classe media vs la vecchia classe media). Così come, a Trinidad, le persone si servono maggiormente della visibilità su Facebook per affermare la ricchezza, altri trovano modi creativi e umoristici per ridicolizzare i loro tentativi in quanto semplicemente volgari e sempliciotti. Denigrare il gusto scorrendo gli account Facebook degli altri è parte sostanziale dell'intrattenimento dei trinidadiani oggi.

Possiamo vedere questi limiti tornando al caso di Sandra. La sua capacità di usare i social media per creare una nuova identità visiva che mostrasse chiaramente quanto fosse riuscita ad ottenere, funzionava in relazione alla gran parte di questo sito, composto da popolazione a basso reddito. 
Tuttavia, esiste una parte di villaggio nuova, gentrificata, un'area con un resort turistico di fronte all'oceano, distante solo un kilometro circa dal centro del villaggio dove vivono i lavoratori manuali sotto pagati come Sandra. Nonostante questa vicinanza, il contatto quotidiano fra questi due mondi capita quasi esclusivamente in conseguenza di relazioni di lavoro: un gruppo lavora per l'altro. I datori di lavoro più ricchi, sia online sia offline tendono a stare in contatto solo con coloro che condividono la stessa appartenenza di classe e perciò lo stesso gusto. Vedono i loro valori in contrasto con quelli degli impiegati paesani, che descrivono come grossolani, ignoranti e persino eccessivamente fissati con il sesso e eccessivamente religiosi.

Queste distanze sociali, che nella regione derivano da secoli di lavoro basato sulla schiavitù, restano come naturalizzate e quindi indiscusse. Solo di recente questi abitanti più ricchi hanno anche riconosciuto che nella regione $\mathrm{i}$ social media sono ugualmente popolari fra le famiglie sottopagate, e tuttavia non hanno alcuna voglia di fare amicizia con i domestici che lavorano per loro. Online questi locali abbienti possono anche condividere fra loro visioni sociali e politiche progressiste, ma questo raramente risulta nel trascendimento dei confini sociali fra loro e i loro vicini meno benestanti. Danno invece voce a temi ecologici, spesso lamentando che la problematica degli accordi salariali al ribasso influenza negativamente l'ambiente o richiede accresciute misure di polizia per prevenire il crimine. I social media, quindi, possono pure aver cambiato la posizione di Sandra in relazione ai suoi pari, ma non avranno alcun impatto su questo più ampio chiasmo sociale fra la sua parte di villaggio e quella dove risiedono i datori di lavoro.

Analogamente, in Italia molti utenti dei social media appartenenti alle élite sono impegnati attivamente in politiche progressiste attraverso Twitter o Facebook, dimostrando la loro simpatia per posizioni di sinistra. Come in Brasile, tuttavia, questo raramente si connette a una qualsiasi azione pratica che potrebbe ridurre la disuguaglianza nel posto in cui vivono. In Italia "curarsi dei poveri” è una cultura in sé e per sé che è facile esprimere sui social media, ma che può avere molto poco a che fare con le reali persone povere della regione.

Nello stesso modo in cui "curarsi dei poveri" segnala una certa posizione di classe in Italia e in Brasile, rappresentarsi come cosmopolita o internazionale raggiunge un obiettivo simile in altri siti. In India del sud le divisioni sociali tradizionali sono rigidamente conservate e controllate online, in particolare mediante il contenuto che le persone condividono. Su Facebook 
gli individui più ricchi condividono articoli, spesso in inglese, e prodotti da media internazionali come The Guardian o The New York Times. Sia i dirigenti IT usciti dal college sia i tradizionali abitanti del villaggio hanno un forte interesse nel cinema, nella politica e nel cricket locali. E probabile tuttavia trovare equamente distribuito nelle timeline dei più ricchi e dei più poveri solo il contenuto relativo al cricket. Le news di cinema e di politica sono differenziate a seconda della casta e della classe, così che gli abbienti postano di arte e cinema internazionale e di Hollywood mentre le persone a basso reddito postano clip di film tamil. Il principale impatto dei social media sta nell'ampio uso di rivendicazioni di cosmopolitismo che enfatizzano le differenze sociali precedenti.

$\mathrm{Nel}$ sito di Trinidad anche le giovani donne professioniste all'inizio dei loro 20 anni, con diploma universitario, condividono immagini sui social media che mostrano influenze globali. Queste immagini riguardano elaborate pietanze servite in ristoranti di alta classe e fotografie di vacanze internazionali ${ }^{29}$. Altri riferimenti riguardano il contenuto di fashion blogger e "vloggers" di YouTube ${ }^{30}$ con base negli USA, in Gran Bretagna, a Singapore. Queste risorse online le aiutano a modellare un senso di cosmopolitismo basato sullo scambio di riferimenti alle tendenze globali in fatto di bellezza, consumi e stile di vita. I post sui social media rendono ancora più visibile l'accesso differenziale a tali esperienze internazionali che questi individui hanno. Questa stessa classe più alta cerca di evitare forum online che discutono di relazioni sessuali e romantiche, un interesse e una pratica centrali per gli abitanti di Trinidad di basso reddito. Postano, invece, materiali sull'essere vicini alla propria famiglia, a sostegno di un matrimonio di lunga durata e del supporto che si può ottenere da un partner. I confini sociali sono anche resi espliciti dall'uso online di espressioni dispregiative: per esempio "ghetto" si riferisce a persone che mostrano attributi come volgarità, mancanza di gusto e scarsa educazione formale, tutti associati agli afro-trinidadiani di basso reddito. Nel caso di Trinidad, tuttavia, troviamo notevole evidenza del modo in cui i gruppi a basso reddito contestano questi tentativi di mantenere la separazione mediante la manipolazione del gusto. Molte frasi e gesti umoristici e ironici sui social media sono esplicitamente finalizzati a punzecchiare le pretese e le affermazioni fatte da coloro che pensano di essere più sofisticati.

Trinidad ha sempre avuto una potente pressione sottotraccia di egalitarismo, che ha le sue specifiche armi in questa lotta. Se i social media fa- 
voriscono una visibilità che consente ai più ricchi di rappresentare la loro ricercatezza, sono anche diventati un sito chiave per l'umorismo, i memi e l'intrattenimento. Una delle versioni più comuni di questo è un'ampia gamma di materiale divertente finalizzato a screditare le pretese di questi cosiddetti cosmopoliti e a canzonare l'“arroganza" di coloro che sembrano "pensare di essere migliori di chiunque altro". Questo è quanto tocca alle élite per il fatto di canzonare la volgarità dei loro inferiori sociali, l'altra faccia dell'intrattenimento prima menzionato.

Il sito cileno, situato nel nord del paese, è caratterizzato da un certo genere di disuguaglianza. La regione è molto ricca di risorse minerali, che si traducono in una grande quantità di ricchezza per le classi elitarie del paese nel suo complesso. Tuttavia, nella specifica città del campo di ricerca la maggior parte è composta da lavoratori manuali sottopagati, che non ricevono granché di benefici finanziari dalle miniere da cui estraggono i minerali. Benché il lavoro regolare nelle miniere sia facilmente disponibile, e sia pagato più di altri lavori, la popolazione locale continua a considerarsi come marginalizzata e sfruttata dalle compagnie internazionali e dal governo nazionale che ne traggono la gran parte dei benefici.

Questo era un altro sito in cui alcuni avrebbero mostrato sui social media foto di beni di lusso, vestiti di marca o vacanze al fine di rendere visibile la loro nuova ricchezza. Questo però distrugge la più larga solidarietà della regione locale ${ }^{31}$ che si definisce in relazione alla nazione nel suo insieme. $\mathrm{Ne}$ risulta che alcune persone erano rappresentate come egoiste, per il fatto di non condividere la loro ricchezza, e sciocche, per il fatto di spendere il loro denaro insensatamente, ed erano persino fatte oggetto di dicerie su loro possibili coinvolgimenti nel mercato della droga. Si riteneva che le persone troppo rapide nel mostrare ricchezza sarebbero veramente state meglio in una città più grande, dove quelli attorno a loro valutavano i beni materiali al di sopra della mentalità comunitaria. Esse sarebbero dunque state escluse rispetto ai confini sociali costruiti attorno a un'ideologia di reciproca solidarietà e aiuto.

Usare i social media come un metodo per legarsi agli altri tramite l'umorismo è più popolare che l'ostentazione della ricchezza individuale. Dalla perdita dei diritti civili in politica fino alle frustrazioni per non essere in grado di pagare la bolletta del telefono o di comprare un pasto particolare come sushi, in forma di memi, fotografie e testi finiscono su Facebook rubricati come "fa ridere quanto sono povero". Anche gli abitanti relativamente be- 
nestanti di Alto Hospicio postano questo genere di memi e testi comici su quanto è divertente essere poveri, riconoscendo questa solidarietà più estesa basata sull'idea che, nel grande schema delle cose, nessuno è privilegiato. Questo a sua volta significa alimentare un certo tipo di orgoglio per la propria marginalità collettiva.

Benché queste lamentele siano di solito finalizzate semplicemente a far ridere il pubblico locale, nell'aprile del 2014 un terremoto di magnitudine 8.2 in scala Richter colpì la regione e il pubblico cui rivolgersi cambiò. Nelle settimane e anche nei mesi che seguirono al terremoto, i social media - in primis Facebook e Instagram - divennero spazi dove concentrare l'attenzione sulla difficile situazione delle vittime e per evidenziare l'assenza di aiuto da parte del governo nazionale. In particolare, più di 4000 famiglie furono lasciate senza casa e vissero nelle tende per quasi due mesi prima che l'agenzia governativa preposta provvedesse a fornire loro case provvisorie. Durante questo tempo, i post sui social si trasformarono rispetto alla consueta forma di socialità interna alla comunità al fine di attirare l'attenzione sull'emergenza delle vittime, e così fare pressione sul governo nazionale per ricevere risorse.

\section{Conclusione}

Come mostrato nella nostra rassegna della letteratura, è necessario fare attenzione a ciò che si intende per disuguaglianza e a come si generalizza al riguardo. È necessario anche differenziare due potenziali conseguenze dell'accesso ai social media: l'uguaglianza che questo rappresenta in sé e il conseguente potenziale impatto su più ampie forme di disuguaglianza. In un certo senso abbiamo prova del fatto che i social media hanno creato una forma di uguaglianza. Il possesso di uno smartphone e l'accesso ai social media da parte di una vasta popolazione di persone a basso reddito in posti come il Brasile, la Cina e l'India rappresenta un profondo cambiamento nelle loro vite. Ora possiedono dispositivi estremamente sofisticati, spesso identici a quelli usati dai ricchi. Ci sono molti esempi descritti in questo libro che dimostrano come, per questo, essi sono in grado di fare molte cose che prima non erano possibili. Sarebbe dunque del tutto sbagliato negare o ignorare questa forma di uguaglianza. Eppure la principale preoccupazione di questo capitolo ha riguardato una questione più complessa: qual 
è la conseguenza di questa eguaglianza online rispetto alla disuguaglianza offline? Come la maggior parte degli esempi ha mostrato, il possesso di uno smartphone e l'accesso o anche la capacità d'uso dei social media non sono assolutamente garanzia di un qualsiasi cambiamento nella disuguaglianza offline; potrebbe diminuire, ma allo stesso modo potrebbe aumentare.

Questo è uno dei capitoli che più chiaramente giustifica la nostra scelta del titolo per il volume. Invece di considerare semplicemente il modo in cui i social media cambiano il mondo, abbiamo posto l'accento sul modo in cui il mondo ha diversificato i social media. Via via che diventano sempre di più parte integrante delle nostre vite, essi finiscono con il riflettere la diversità culturale del nostro mondo. Più in particolare, la relazione fra social media e mobilità sociale è estremamente diversa quando vista lungo tutti e nove i siti.

Questo riflette più a pieno la precedente letteratura su questo argomento, caratterizzata inoltre da un'enorme varietà di posizioni dalle più ottimistiche alle più pessimistiche. Così, in qualche modo, la nostra conclusione è a supporto di questo ampio spettro contemplato in letteratura, ma per suggerire infine che piuttosto che andarle incontro come una lotta fra generalizzazioni globali riguardo a un presunto impatto generale, sarebbe meglio riconoscere che gran parte di queste istanze sull'impatto dei social media sull'uguaglianza sono probabilmente appropriate, anche se in riferimento a differenti regioni e popolazioni.

Uno degli esempi più semplici di come il mondo ha cambiato i social media si verifica nel caso dell'India del sud. Qui strutture sociali altamente rigide e gerarchiche sviluppatesi nel corso di molti secoli, a loro volta hanno colonizzato questi nuovi media facendo sì che essi riflettono queste distinzioni. I social media hanno un impatto soprattutto nella più grande enfasi su rivendicazioni differenziali di cosmopolitismo, che possono essere sostenute da azioni di condivisione di materiale internazionale trovato online. Abbiamo trovato questa enfasi crescente sul cosmopolitismo in gran parte dei nostri siti, dove essa in genere esaspera disuguaglianze preesistenti. Un problema simile si presenta nel nostro sito in Italia. Qui i social media sono percepiti come l'obbligo oppressivo a prendere parte a qualcosa che ti costringe a essere ancora più consapevole della bassa posizione sociale. In tutti questi casi i social media rendono la disuguaglianza più visibile e radicata.

Quando ci siamo dedicati alla relazione fra social media, istruzione e mobilità sociale abbiamo trovato però alcuni casi estremamente diversi. Come 
indicato nel Capitolo 5, l'attenzione al ruolo dei social media in relazione a un elevato impegno verso l'istruzione formale nella Cina rurale è molto diversa dall'uso dei social media nell'apprendimento informale. Questo forse perché le persone non si preoccupano tanto dell'istruzione formale, come nel caso della Cina industriale, o perché non possono avere accesso a una istruzione formale di qualità decente, come nel sito brasiliano. L'uso dei social media come una risorsa per l'istruzione informale è probabilmente la componente aggiuntiva più importante sottolineata in questa prima sezione, che aiuta le persone a lottare contro la disuguaglianza.

Per gran parte del capitolo abbiamo visto una tensione fra due proprietà dei social media tra quelle più comunemente osservate. La prima riguarda l'aumento della visibilità che essi consentono, ma la seconda, decisamente importante quanto la prima, è l'uso dei social media come luogo di umorismo e di ironico dileggio. Nel caso di Sandra e in quello del Brasile abbiamo visto che la visibilità può cambiare la propria posizione sociale dimostrando la propria rispettabilità, e perciò può essere uno strumento di mobilità sociale. Eppure, per converso, abbiamo scoperto i limiti di questo processo, dato che nessuno di questi obiettivi è raggiunto in relazione a una più estesa distinzione sociale che includa datori di lavoro e impiegati. L'uso dei social media per esprimere attenzione verso i poveri, di fatto, è emerso come uno dei fattori chiave di separazione della ricchezza dai poveri.

A Trinidad come in Cile abbiamo visto il modo in cui le persone usano la visibilità per cercare e rappresentare cosmopolitismo e ricchezza, contrastato dall'uso dell'umorismo per bucare queste bolle di pretenziosità e spingere per un'agenda più egualitaria. Nel caso finale del Cile, tuttavia, abbiamo visto anche che, come in precedenza nel caso brasiliano, molto dipende da come si mette a fuoco o si assume una prospettiva grandangolare rispetto al panorama più ampio. Quando le persone di questo luogo considerano se stesse in relazione alla nazione più grande, come sono state costrette a fare per il terremoto, le divisioni interne diventano meno importanti rispetto alla più grande relazione con la nazione o con i contesti internazionali in cui tutti loro vivono.

Per concludere, questo capitolo ha mostrato la difficoltà di fare affermazioni rispetto all'impatto dei social media sulla disuguaglianza. Chiaramente non vogliamo contribuire alla disuguaglianza venendo meno al rispetto dei diversi modi in cui le persone, in ogni sito, concepiscono le differenze sociali e ne fanno esperienza. Nello stesso tempo, c'è un'altra questione delicata, 
ugualmente importante, che consiste nel non mettere insieme la parità di accesso con qualsiasi presunta conseguenza, dato che una delle nostre osservazioni più generali riguarda il fatto che il grande incremento nell'accesso ai social media non è di per sé garanzia di cambiamento in altre forme di disuguaglianza sociale. 


\section{Capitolo 10 \\ Politica}

Nel dibattito accademico esiste un problema del tutto ragionevole circa il grado in cui i social media stanno trasformando la politica, intesa come istituzioni di governo con tutti i dibattiti e i conflitti ad esse relativi. Nello studio etnografico, tuttavia, questo non è il nostro focus privilegiato. Cerchiamo invece di derubricare la politica semplicemente a ciò che concretamente emerge dall'osservazione dei social media utilizzati dai nostri informatori. Se si cerca il dibattito politico lo si troverà, ma ciò non consentirà di valutarlo in modo imparziale come un elemento della vita quotidiana delle persone in luoghi specifici. In effetti i nostri casi di studio aperti rivelano che nei siti maggiormente dominati da conflitti politici, la politica, proprio per questo, può non essere dominante sui social.

Mardin, nella Turchia sud orientale, ha avuto una lunga storia di conflitto e violenza politica. Vicino al confine siriano, i vari gruppi etnici, linguistici e religiosi che vivono nella cittadina si sono scontrati in varie occasioni. Per Costa, che ha avuto un particolare interesse verso l'impegno politico nell'area, Mardin come campo di ricerca era una scelta obbligata. Ha tuttavia trovato che la gente di Mardin evitava di discutere apertamente o comunque in luoghi pubblici di politica e di altri argomenti sensibili. Per lo più si parlava di politica a casa, con familiari o amici stretti con cui si condividevano opinioni e valori. Sui social media, e su Facebook in particolare, c'erano pochi riferimenti alla politica, specialmente quella locale. Durante le infuocate campagne elettorali in vista delle elezioni locali del marzo 2014, alcuni sostenitori ruppero il silenzio e si unirono alla raccolta pubblica di sostegno al loro partito politico. Anche in quell'occasione, però, su Facebook poche persone condivisero post sulle loro pagine personali. Al massimo un gruppetto seguì le pagine di politici locali e mise "mi piace" ad alcuni dei loro post.

Facebook è usato primariamente per mantenere buone relazioni con amici e parenti, quindi il silenzio pubblico sui temi politici locali è importante per la coesistenza pacifica sia online sia offline. Una certa attività politica come quella presente sui social media di solito riguarda il livello nazionale ed è condotta maggiormente da parte dei sostenitori del governo in carica, 
dato che le persone sono consapevoli del controllo statale on line. Questi sostenitori governativi possono esprimere solidarietà nei confronti dell'AKP al governo e del primo ministro, Recep Tayyip Erdogan. Durante le proteste al Gezi Park nella primavera e nell'estate del 2013, i sostenitori dell'AKP condivisero memi, video e news che riproducevano la propaganda statale e screditavano gli attivisti, agevolati dall'impegno attivo del governo sui social media. Nel settembre del 2013 l'AKP, a quanto si dice, riuscì a reclutare oltre 6000 utenti di social media per influenzare l'opinione pubblica ${ }^{1}$.

$\mathrm{Al}$ di là della politica locale, la popolazione curda di Mardin usa Facebook per esprimere solidarietà verso i Curdi in Siria e in Iraq, specialmente nella battaglia contro il cosiddetto Stato Islamico (IS). Nella misura in cui il tema politico era esterno alla Turchia si sentivano liberi di essere fortemente attivi sui social a sostegno del popolo curdo residente in queste nazioni vicine. Molti cambiarono l'immagine del profilo con il termine Rojava ("Occidente"), con riferimento alla regione libera curda nel nord della Siria. Quando lo Stato Islamico (IS) occupò Sinjar nell'Iraq curdo, la popolazione curda di Mardin usò Facebook per mostrare sostegno e organizzare una raccolta di fondi, vestiti e viveri da distribuire ai rifugiati yazidi che erano arrivati in città. Analogamente, quando l'IS occupò Kobane, nella regione di Rojava, molti curdi di Mardin postarono memi, immagini e news in segno di solidarietà con la popolazione sotto assedio. Benché avessero trovato lo stato turco complice di questi avanzamenti, di nuovo evitarono di postare materiale esplicitamente di condanna del ruolo dello stato. In generale, per questo, i social media rafforzano le condizioni preesistenti della politica nella città, incluse l'assenza di discussione sulla politica locale e sulla soppressione di posizioni di opposizione, ma favoriscono l'espressione di solidarietà curda ai curdi in altri stati.

L'enorme letteratura disponibile su internet e politica, e più specificamente su politica e social media, è cambiata nel tempo. Iniziò con un focus sul ruolo di internet nei nuovi movimenti sociali negli anni Novanta ${ }^{2}$ e fu seguita dal problema del "digital divide" e dell'"e-governance" ${ }^{3}$, il ruolo delle piattaforme Web 2.0 e del contenuto generato dagli utenti ${ }^{4}$. Studi più recenti hanno esaminato le conseguenze delle affordance del WIFI e dei media mobili come gli smartphone, in modo particolare in riferimento al loro ruolo nell'organizzare l'attività politica collettiva ${ }^{5}$. Chadwick e Howard presentano un volume eccellente sul dibattito critico sulle relazioni tra internet, politica statale e cittadinanza, mentre Postill sintetizza la ricerca chiave 
sulla politica digitale e i modi in cui l'analisi etnografica contribuisce alla comprensione dell'ecologia dei movimenti di protesta ${ }^{6}$. Agli inizi degli anni 2000 si ebbe un evidente sentimento di ottimismo riguardo all'e-governance e all'e-government, e al potenziale che entrambi presentavano al fine di ridurre il "digital divide" in procinto di trasformare la "sfera pubblica", un concetto legato a teorici sociali come Habermas ${ }^{8}$. Più recentemente, l'attenzione si è rivolta al ruolo dei social media nell'organizzazione dell'azione politica, in particolare nelle diverse esperienze regionali della "Primavera araba" . Ciò, in un certo senso, ha rappresentato il punto di svolta di tali studi, sollecitato dal lavoro di Morozov sull'uso delle tecnologie digitali nella repressione politica durante questi eventi. Da allora c'è stato un corpus crescente di ricerche critiche sull'assunto che il ruolo principale delle tecnologie digitali sia quello di aumentare in modo significativo la partecipazione democratica ${ }^{10}$. In ogni caso, c'è anche un interesse notevole nell'uso dei nuovi media come basi per forme alternative di azione collettiva, per esempio le ricerche dell'antropologo Coleman su Anonymous e altri attivisti politici online ${ }^{11}$.

In quanto studio antropologico, in questo capitolo piuttosto che applicare una qualsiasi definizione data relativa al processo democratico, alla società civile o alla governance ${ }^{12}$, per "politica" si intende tutto ciò che è ritenuto "politico" dai nostri informatori attraverso tutti e nove i siti di ricerca. Lo stesso varrà per la partecipazione politica. Molti ricercatori con un interesse per la politica sono attratti da situazioni ritenute significative in termini politici o con un elevato livello di attività politica, specie quella che potrebbe risultare dell'uso dei social media ${ }^{13}$. Con l'eccezione di Mardin selezionata da Costa, tutti i nostri campi di ricerca invece sono stati scelti proprio perché "normali". Il risultato è che noi siamo in grado di valutare il grado di concreta attività politica al di là degli stereotipi creati dalla scelta di campi con l'esplicito scopo di studiare la politica.

La nostra principale scoperta è stata l'ampia diversità nel modo in cui i vari significati di "politica" si intrecciano con i social media attraverso i nove siti, benché, in generale, la nostra ricerca mostri che i social media stanno avendo meno impatto di quanto si sarebbe dedotto dalla letteratura precedente $^{14}$. Saremmo tuttavia concordi nel ritenere che questa è una scoperta altamente significativa, poiché è altrettanto importante accertare il grado in cui i social media sono coinvolti nella politica per le persone comuni nelle situazioni più tipiche quanto lo è lo studio del ruolo dei social media in 
momenti e luoghi più politicamente densi.

Come abbiamo rilevato in posti come Mardin, generalmente i social media riflettono le strategie della discussione e dell'assenza di discussione politica che si erano sviluppate nel mondo offline. Comunemente abbiamo lavorato in campi dove l'impegno politico per gran parte delle persone è limitato e spesso indiretto. Ci sono molte ragioni per cui il dibattito politico offline può non riprodursi online, tra cui sentimenti di indifferenza, disillusione o apatia, o anche il non voler essere visti dagli altri come "politicamente impegnati”. Inoltre, le persone nel nostro sito in Italia del sud prevalentemente hanno espresso disillusione verso la corruzione dei politici nazionali. Nel sito inglese prevalentemente si è coinvolti con la politica come fonte di umorismo, mentre nel campo di ricerca a Trinidad le persone erano attirate dai temi politici soprattutto quando questi davano spettacolo o suscitavano scandali.

Nonostante questa diversità nel modo in cui le persone sono coinvolte con la politica sui social media, la nostra ricerca ci ha condotto a una conclusione generale che sembra applicarsi a tutti e nove i siti. La partecipazione politica sui social media in cittadine piccole, medie e semi-urbane è enormemente influenzata dalle relazioni sociali all'interno di questi posti relativamente piccoli. Gli informatori erano preoccupati nel mantenere o rafforzare relazioni con i loro contatti sui social, e non volevano rischiare di danneggiare amicizie o relazioni con familiari o colleghi. Persino in nazioni come la Turchia e la Cina, dove lo stato controlla più esplicitamente e sistematicamente gli usi dei social media, non è solo tale controllo in sé a influenzare maggiormente l'espressione politica. Piuttosto è il modo in cui il potere dello stato si manifesta attraverso le norme sociali che determinano le relazioni fra gli individui ${ }^{15}$. Questo capitolo al riguardo identifica tre tematiche: come le relazioni sociali impongono norme che sono valide sui social media; come il controllo statale e la politica nazionale permeano la discussione e in modi diversi arrivano a includere il modo in cui le persone si esprimono sui social media; e come, in conseguenza di queste ultime due pressioni, la partecipazione politica generalmente assume forme diverse sui social media. 


\section{Politica}

\section{L'attenzione alle relazioni sociali rende i social media un posto conservatore}

In modo simile a quanto argomentato nel capitolo precedente con riferimento al genere, le concezioni politiche personali in spazi pubblici (o semi pubblici) come Facebook sono espresse in un modo che protegge le relazioni sociali delle persone e la loro reputazione personale. Potrebbero fare eccezione piccole minoranze che si identificano con il loro essere attivisti politici, per esempio studenti universitari militanti o membri di partiti politici locali, ma queste nei nostri siti rappresentano una piccola minoranza. D'altra parte, i social media sono in gran parte usati nel tentativo di aumentare il proprio status sociale o la propria popolarità, di affiliarsi a gruppi ritenuti di prestigio o semplicemente di mantenere buone relazioni con gli altri. La stessa osservazione si potrebbe fare a proposito delle interazioni quotidiane offline, laddove le persone tendono a stare attente a discutere di politica con coloro che hanno punti di vista radicalmente diversi, o a provocare coloro che fanno parte della loro cerchia sociale ma hanno posizioni politiche diverse. Noelle-Neumann ha coniato l'espressione la "spirale del silenzio" per descrivere questo fenomeno e il timore delle persone che la politica possa condurle a essere isolate o ostracizzate ${ }^{16}$. Ciò è evidente in modo particolare sui social media, a causa della loro visibilità amplificata. Al contrario, i social diventano uno spazio per esprimere idee e valori condivisi.

Per esempio, nel nostro sito dell'Italia del sud, per quanto sia frequente essere amici con politici di differenti gruppi politici, gli individui sono estremamente cauti nell'impegnarsi politicamente in uno spazio così visibile come Facebook. Sarebbe altamente inusuale mettere un "mi piace", commentare o condividere un qualsiasi post politico relativo a un politico locale. Sono invece i politici locali a condividere prontamente qualsiasi tipo di pubblicità positiva sui media locali e a costruire lunghi aggiornamenti di stato su Facebook per descrivere qualsiasi risultato ottenuto nell'arena locale. Invece di impegnarsi in questo genere di cose, le altre persone usano i social media per indirizzare critiche verso figure pubbliche che rappresentano l'“Europa”, lo "stato", e la "Regione", o per riferirsi a temi generali con cui ciascuno di quelli che conoscono sarebbe d'accordo, per esempio la disoccupazione, la corruzione o l'inefficienza del governo.

La situazione è simile nel sito dell'India meridionale. Gli utenti dei social media raramente postano un contenuto serio relativo alla politica locale che 
possa essere visto dai loro pari, e potrebbe di fatto provocare una reazione negativa da parte degli stessi politici locali. Le critiche sullo stato della politica e del governo locali hanno luogo invece nelle conversazioni private. I soli a essere politicamente attivi sui social media sono coloro che lavorano per i partiti politici locali; i loro post sono chiaramente finalizzati a conquistare sostegno evidenziando alcuni eventi o alcune azioni positive. Tali post attirano i "mi piace" e i commenti solo da parte di amici stretti o di altri che lavorano nel partito. Molti di questi attivisti appartengono a una classe socio-economica bassa o vivono in zone rurali, e i loro post raramente potrebbero essere visti dai membri delle nuove classi medie e medio-alte delle aree urbane. Per il resto, su Facebook c'era molto poco impegno rispetto alla politica locale. I post sulla politica a livello nazionale o statale, visti come espressione di un'opinione politica più ampia, erano più comuni. In entrambi i siti dell'India del sud e brasiliano c'è il sincero timore delle conseguenze negative dirette per sé e per i propri familiari se si dovessero sviluppare rapporti ostili fra membri di partiti rivali.

Parallelamente a questo esiste la commisurata attenzione a mantenere buoni rapporti con i leader e i partici politici locali. Un altro fattore potrebbe riguardare situazioni in cui i parenti fossero attivisti di politici o di partiti diversi, il che renderebbe le persone preoccupate che postare su temi politici locali possa avere ripercussioni per questi loro parenti. Nell'agosto del 2014, durante la campagna elettorale brasiliana, i veicoli di propaganda viaggiavano per diversi villaggi, diffondendo al megafono slogan e motivetti. Gruppi di persone reagivano alla loro presenza con brevi conversazioni, ma non c'erano commenti politici online se non per esprimere sostegno o critica a partiti di livello statale o nazionale.

Nella Cina rurale questa tendenza assume una forma diversa. Tema politico ammissibile è la corruzione, che è anche la questione più pressante per i residenti dell'area. Invece di esprimere le loro opinioni apertamente online, però, gli interessati cercano di sistemare le loro situazioni personali mediante approcci discreti con gli individui che ritengono abbiano più potere $\mathrm{e}$ influenza. Qualora questa strada si riveli fallimentare, le persone potranno prendere in considerazione la possibilità di fare una petizione a un livello più alto, ma quasi mai daranno voce alle loro lamentele o esprimeranno le loro frustrazioni sulle piattaforme di social media. Quando il tema della corruzione appare online, si tratta di corruzione in altre città $\mathrm{o}$ in altre parti del paese. 
Tutto questo significa che i social media visibili al pubblico sono generalmente diventati un luogo altamente conservatore, che riflette i valori e le norme sociali prevalenti che regolano le relazioni fra le persone a livello locale. Se non altro, la politica online è sfruttata come fonte di spettacolo per aumentare la popolarità tra gli amici o per creare nuove, utili connessioni sociali. Ciò è particolarmente evidente nel sito inglese, dove le discussioni politiche sui social media prendono in gran parte la forma di scambio di battute online fra amici. Meme e commenti più seri si trovano di più o tra i gruppi di livello economico più alto che si schierano a favore di temi ambientalisti o fra i gruppi a basso reddito che vivono nelle case popolari e sostengono cause nazionaliste come dare supporto all'esercito o mettere al bando gli immigrati. A parte questi, il modo predominante è l'umorismo a spese dei politici. La politica sui social media è utile per lo più per divertire e stringere legami con amici.

Infine, il sito della Cina industriale offre una variazione su questo tema delle relazioni personali. Ci sono qui esempi di social media usati con l'intenzione di rafforzare strategicamente le "guanxi" ("relazioni strumentali"). Lazienda proprietaria dei profili WeChat mostra che le persone scelgono di non postare niente sulla politica per evitare di mettersi in situazioni potenzialmente rischiose. A volte invece usano i social media per coltivare relazioni con funzionari del governo locale, nella speranza che queste amicizie li aiuteranno a tenere buoni contatti con il governo per potenziali benefit per i loro affari.

\section{Controllo statale e politica nazionale}

Per coloro che risiedono in molti dei nostri siti di ricerca l'attenzione alle relazioni sociali ha costituito un deterrente nell'esprimere un disaccordo, nel discutere o nel manifestare opinioni politiche, specie in riferimento alla politica locale. Questo paragrafo prende in considerazione un altro aspetto che dà forma alla partecipazione politica sui social media, rendendo questi spazi online radicalmente diversi rispetto al modello habermasiano di "sfera pubblica", l'ambito in cui le persone possono discutere liberamente di argomenti e interessi comuni al fine di raggiungere un comune accordo ${ }^{17}$.

In Cina e in Turchia lo stato controlla gli usi dei social media e i contenuti cui si può avere accesso tramite internet. Il controllo statale è una pressione 
potente che ha influenzato i modi in cui gli spazi semi-pubblici dei social media sono utilizzati.

Nei due campi cinesi non c'erano prove di una preoccupazione diffusa relativa alla censura statale dei social media. Il controllo del governo centrale su internet è stato non solo di ampio raggio ma anche particolarmente preciso e sofisticato, comparabile alla diretta coercizione, e alle costrizioni, dei cittadini. Come esito della storia dei media locali e della loro posizione rispetto ad altre porzioni di vita, i residenti di quei siti semplicemente non concepiscono i social media come un ambito appropriato o potenziale per discutere di politica e criticare il governo centrale. Al contrario, considerano le piattaforme di social del tipo QQ e WeChat alla stregua di posti per divertirsi, creare nuove relazioni e rafforzare quelle già esistenti. Questa percezione è comune sia alla Cina industriale che a quella rurale.

Questa assenza di critica verso il governo o di qualsiasi altro tipo di impegno politico online appare più significativa se confrontata con il forte interesse delle persone per la discussione di argomenti politici offline: intensi dibattiti spesso si svolgono a cena o all'ora del tè. Il potere dello stato cinese si esprime anche nell'uso dei social media per fini propagandistici, mediante la distribuzione di nuove narrazioni che spesso seguono o riproducono la linea ufficiale del partito su argomenti diversi. Sia QQ sia WeChat diffondono tre news report al giorno, prodotti dalle aziende stesse di social media. C'è la percezione generale che queste news siano utili, benché ci sia anche una certa consapevolezza critica verso l'inclinazione delle news a favore dello stato. La propaganda statale inoltre diviene incorporata e riprodotta da parte degli utenti dei social media, come è evidente nell'abbondanza di post nazionalisti come quelli che diffondono sentimenti anti giapponesi o ammirazione per i leader del partito centrale.

Il risultato è che non occorrono azioni specifiche di coercizione. I ricercatori in Cina non si sono trovati in mezzo ad alcun caso di repressione o restrizione negli usi dei social media nei loro rispettivi siti. Ci sono stati pochi casi di critica individuale verso il governo centrale nel sito della Cina industriale, ma questi non hanno avuto come riscontro alcuna ripercussione del tipo esplicita messa al bando dell'account o altre misure punitive. Questi esempi allora supportano le scoperte di studi precedenti, che sostengono che la censura in Cina è maggiormente diretta a prevenire qualsiasi azione collettiva piuttosto che a sopprimere critiche individuali ${ }^{18}$.

$\mathrm{Al}$ di fuori della più ampia sfera politica, McDonald ha osservato altri casi 
di resistenza quotidiana attraverso l'aggiramento delle restrizioni imposte dal governo. Per esempio, per tutta la Cina, c'è il divieto per i giovani sotto i 18 anni di usare internet caffè e agli utenti viene richiesto un documento di identità prima di consentire il collegamento. Nell'unico internet caffè nel campo di ricerca, tuttavia, il gestore teneva una riserva di carte d'identità in più (prestate da amici e membri della famiglia estesa) per prestarle agli utenti minorenni così che potessero connettersi.

Questi utenti minorenni erano fatti accomodare in due stanze "segrete" nel retro del caffè, lontano dalla strada e quindi fuori dalla vista di altri del posto e di ispettori. Nonostante solo il due per cento degli studenti di scuola media presi in esame identificasse l'internet caffè come il posto principale per usare internet, il tipo di clientela più comune nel caffe sembrava essere rappresentata proprio da questi studenti.

Nella Turchia sud orientale i curdi e i dissidenti di sinistra hanno sofferto le conseguenze delle violenze sancite dallo stato in diverse recenti occasioni. Durante gli anni Ottanta e Novanta del Novecento, arresti e torture, sparizioni e assassinii riguardarono gli attivisti politici, e altre forme di repressione, per esempio il divieto di parlare in curdo, erano parte della vita quotidiana. Oggi i simpatizzanti del movimento curdo $^{19}$ sono apertamente e pubblicamente restii a criticare uno stato che è chiaramente intenzionato a usare la violenza contro di loro. In questo contesto la censura di internet è un'altra espressione del potere e della violenza dello stato, e in questo modo determina l'uso dei social media.

A differenza del caso cinese, gli abitanti di Mardin sono spesso stati oggetto diretto di divieti e blocchi; pagine su Facebook che sostengono quello che all'epoca era il Kurdish Peace and Democracy Party (BDP) in certi casi sono state chiuse; inoltre, alcune persone sono state incriminate per diffamazione per aver criticato personaggi potenti. Gli oppositori al governo generalmente sentono la pressione di essere osservati e sotto sorveglianza. Come abbiamo visto, ciò ha portato alla soppressione della discussione politica aperta online, dato che essa era stata già in precedenza repressa offline. I social media portano a un intreccio tra la struttura sociale e la politica, fino al punto che il controllo statale si sovrappone alla sorveglianza sociale di amici, conoscenti o parenti e ne viene rafforzato. 


\section{Questioni locali di partecipazione politica}

Questo capitolo è iniziato con la discussione del caso di Mardin. Abbiamo visto che in posti dove la tensione politica è forte, postare sui social media è un'azione altamente limitata e cauta. Questo paragrafo descrive altre forme di impegno politico sui social media. Come in gran parte di questo studio, vedremo il modo in cui sono i fattori locali a essere della massima importanza. Ancora, gli esempi sono conformi alla nostra più ampia generalizzazione relativa al fatto che l'uso dei social media è attentamente controllato, in modo da non danneggiare le relazioni social individuali e le reputazioni personali.

Abbiamo visto come nel sito inglese l'umorismo politico è davvero largamente indicato per scherzare fra amici e per mostrare quanto l'individuo sia arguto o spiritoso. Analogamente, nel sito dell'India meridionale il modo più comune di impegno politico sui social media è mediante l'umorismo e il sarcasmo. Come messo in luce all'inizio del capitolo, il commento politico diretto è raro. Tuttavia, il trolling (postare dispregiativo o offensivo indirizzato verso un individuo) contro personalità politiche tamil è diffuso fra coloro che hanno un' età compresa fra i 20 e i 45 anni, e appare sulle timeline di Facebook oppure nelle conversazioni su Whatsapp. Tali post sono indirizzati con attenzione, per lo più ai politici tamil non troppo potenti, e la maggior parte degli osservatori troverebbe questi post divertenti per il loro tono sarcastico. Eppure, le stesse persone che potrebbero far circolare questo tipo di post prenderebbero le distanze da troll particolarmente offensivi o dal linguaggio osceno per puntualizzare qualcosa, ritenendo che tali utenti abbiano spinto lo scherzo troppo oltre.

Solo l'anonimato consente alle persone di esprimersi più direttamente su temi particolarmente sensibili. Diversi troll anonimi su You Tube usano un linguaggio offensivo e esprimono scarso rispetto verso i politici. Alcuni impiegati nell'IT del sito indiano commentano argomenti molto sensibili, per esempio la conversione al Cristianesimo di Hinduisti, il terrorismo islamico o la critica del Pakistan, ma in gran parte sotto falsi nomi per evitare di essere identificati da altre persone. Per esempio Sandeep, un impiegato di 24 anni di una piccola azienda di IT app development, non perde occasione di lasciare un commento sarcastico sul Pakistan e sui suoi legami con il terrorismo islamico usando uno dei suoi sei falsi account. È tuttavia molto serio nel fare attenzione a non svelare la sua reale identità.

Il campo di ricerca a Trinidad offre un altro esempio di come si è svolto online un evento politico. Sinanan descrive uno sciopero della fame da 
parte dell'attivista dott. Wayne Kublalsingh, docente nella University of the West Indies, St. Augustine, Trinidad. Kublalsingh era il leader di un gruppo attivista, l'Highway Re-route Movement (HRM), che protesta contro la costruzione di un tratto di autostrada nel sud del paese. A cominciare dal 2006, un gruppo di abitanti nell'area aveva cercato senza successo di ottenere informazioni e ascolto da parte del governo statale a proposito di ciò che percepivano essere la loro infelice ricollocazione. Quando la costruzione ebbe inizio nell'area coinvolta, Kublalsingh cominciò uno sciopero della fame di fronte all'ufficio del primo ministro, che durò 21 giorni. Il suo scopo era forzare il governo a riconsiderare la propria decisione di costruire questo tratto di autostrada. Anche se la costruzione è andata avanti, le azioni di Kublalsingh ebbero successo nella misura venne avviata una commissione d'inchiesta indipendente che pubblicò poi i risultati.

LHRM, il gruppo attivista alle spalle di Kublalsingh, aveva una pagina Facebook molto attiva che ricevette centinaia di commenti durante lo sciopero della fame. Alcuni erano molto divertenti e altri avevano un contenuto molto serio, ma per lo più erano di persone che non si identificavano come "attivisti". La strategia personale di Kublalsingh era attirare l'attenzione sulla questione mediante l'azione spettacolare dello sciopero della fame. Il suo problema era promuovere la discussione pubblica, a prescindere dal fatto che le persone fossero o meno d'accordo con lui. Lo sciopero della fame era comunque un evento mediatico, post e fotografie del corpo di Kublalsingh che deperiva venivano mostrate sia sui social che sui media mainstream. La gente per questo non solo fu coinvolta nel dramma che si stava svolgendo, ma fu anche più esposta a temi di sviluppo e di governance che ruotavano attorno $\mathrm{al}$ rafforzamento dell'autostrada come progetto statale di larga scala. Dopo che il primo ministro accettò di rivalutare la decisione di costruire quel tratto di autostrada e che Kublalsingh pose termine al suo sciopero, queste conversazioni in gran parte si affievolirono, anche se i circoli attivisti continuarono a postare aggiornamenti su Facebook, blog e piattaforme online indipendenti.

Se però esaminiamo questo evento attraverso le lenti locali del nostro sito a Trinidad, le persone raramente si impegnavano in proposito sui social se non attraverso pochi scherzi o memi, anche se esse esprimevano volentieri le loro idee nelle conversazioni di tutti i giorni. Questo caso illustra due punti a proposito di partecipazione politica e social media a Trinidad. In primo luogo, benché fossero emerse discussioni sui temi importanti dello sviluppo e della governance, queste furono di gran lunga sommerse dallo spetta- 
colo dello sciopero della fame in se stesso. È caratteristico della politica a Trinidad il fatto che ciò che trovò risonanza nelle persone fu la mostra del corpo successiva alla decisione di Kublalsingh di avviare uno sciopero della fame e la performance delle persone legate a questo evento, e non qualsiasi impegno verso i temi ben più profondi che erano contestati ${ }^{20}$. In secondo luogo, il caso ha mostrato che per le persone di una piccola città l'attivismo e la partecipazione politica pubblica sono qualcosa cui la maggior parte della gente non vorrebbe essere associata. Discorsi e ironia sulla politica vanno bene, ma "essere politici", come riflesso in commenti seri sui social media, no. Le persone delle cittadine sono attente a evitare di essere ritenute attivisti politici, un ruolo che percepiscono come proprio di élite metropolitane, studenti universitari o cerchie di artisti. Il punto qui è simile a quello fatto a proposito dell'umorismo in Inghilterra. Scandali, pettegolezzi, o cose che fanno spettacolo sono divertenti e hanno risonanza, per cui questi sono gli aspetti dell'evento politico che concretamente trovano il loro spazio nei social media locali. Il contrasto si nota con ambiti come la morale o la religione, dove le persone si scambiano di norma memi piuttosto seri.

Per capire la situazione nel nostro sito in Cile bisogna di nuovo considerare ciò che intendiamo per "politica". Abbiamo discusso a lungo se la gente si impegna in argomenti di politica locale o nazionale o li evita, ma in questo caso il tema politico centrale era la costruzione di qualcosa che potesse essere considerato come locale, in quanto distinto da nazionale. Come indicato in precedenza, gli abitanti dell'area percepiscono che la regione (che divenne parte del Cile solo nel diciannovesimo secolo) è sfruttata per le sue risorse naturali che sostengono l'economia dell'intero paese, eppure loro sono trascurati dal governo. La maggior parte della gente si sente lontana rispetto alle espressioni della nazione come entità politica. Eppure Haynes nota che questi settentrionali sono molto orgogliosi di essere "culturalmente cileni" allorché si tratta di tifare per la nazionale di calcio, preparare cibo o bevande cileni tradizionali, e addirittura nel discutere rivalità economiche o sociali rispetto alle nazioni vicine. Anche lo slang usato online è unicamente cileno, e difficile da capire per altri parlanti spagnolo. I social media in particolare diventano un luogo dove rivendicare la cilenità mediante riferimenti culturali e linguistici ma anche, nello stesso tempo, criticare il governo nazionale e affermare un regionalismo basato sulla marginalità, utilizzando memi ironici, divertenti. Questo sito rappresenta il nostro caso più evidente di social media come strumento per mettere insieme gli abitanti di una città che si è 
battuta per ottenere maggiore visibilità all'interno della nazione.

Un altro tratto comune della partecipazione politica sui social media, è ciò che può essere chiamato "partecipazione passiva", in riferimento alla tendenza a criticare le cose in un modo più rassegnato. Nella Cina industriale la locale classe media maschile, per esempio i proprietari di piccoli negozi, usavano i social media per prendersi gioco della politica e per creare scherzi innocui sui politici, nel tentativo di apparire divertenti e arguti agli occhi dei loro pari. In modo analogo a quello proprio dei media "più vecchi“, i social erano prevalentemente usati dalle persone comuni per "osservare" la politica, proprio come gli spettatori guardano una partita di calcio, e non per "fare" politica. Come nei casi inglese e di Trinidad, la chiave della relazione dei social media con la politica è che quest'ultima è vista come fonte di intrattenimento. La politica, come lo sport, è qualcosa che offre uno spettacolo banale che le persone possono sfruttare dove e quando decidono.

Lo stesso principio si può applicare all'utilizzo serio tanto quanto a quello umoristico. Nel sud Italia i social media erano diventati il luogo in cui le persone esprimevano frustrazione e collera contro la politica in generale, questioni sulle quali la maggior parte della gente concorda. Non è che Facebook venga usato per protestare o avviare cambiamenti della situazione attuale, nonostante un grande e diffuso malessere circa la condizione della politica contemporanea. Al contrario, le persone usano i social media per criticare una questione ben nota che molti sentono di non essere in grado di modificare. Come per l'umorismo, allora, essere impegnati in politica non corrisponde a "fare" politica, ma ad usarla per scopi locali come motivo di conversazione quotidiana e spesso per esprimere frustrazione ("sfogare"). Il vantaggio della politica è che, come lo sport o le celebrità, offre una cultura comune condivisa.

\section{Conclusioni}

Scopo di questo capitolo è stato esplorare come e perché i social media possono essere impegnati con la politica all'interno di siti di ricerca scelti primariamente per motivi che non avevano nulla a che fare con la politica. $\mathrm{Ci}$ auguriamo che questo possa bilanciare la maggior parte delle discussioni su questo soggetto basate su ricerche in posti dove la politica rivestiva un particolare significato. In generale, abbiamo trovato che, se ci si rivolge a siti di ricerca ordinari, la politica sui social media ha un profilo più basso di 
quanto ci saremmo aspettati. In alcuni casi, perché forse è stata soppressa e questo porta a una rappresentazione altamente conservatrice della vita e delle opinioni delle persone online. In altri casi, tuttavia, è perché i social media sono associati più con l'intrattenimento e la costruzione di legami sociali che non con argomenti seri come la politica.

Queste considerazioni possono essere sintetizzate come le tre principali scoperte che corrispondono alle tre sezioni in cui è diviso questo capitolo. In primo luogo, le relazioni personali costituiscono il fattore maggiore che orienta online l'impegno e l'azione politica. La principale attenzione delle persone riguarda come i loro post avranno impatto sulla loro famiglia e sui loro amici. In diversi dei nostri campi di ricerca le persone sentivano che esprimere opinioni e concezioni politiche poteva risultare antagonista o conflittuale; ne risulta che la politica resta invisibile e la discussione è riservata agli spazi privati con i propri familiari e amici più stretti. Solo in posti dove le persone usano profili falsi e anonimi il commento politico esplicito è frequente. Abbiamo, tuttavia, molti casi di commento politico indiretto, per esempio nel sud dell'India e in Cina i commenti riguardano argomenti nazionali piuttosto che locali.

In secondo luogo le norme sociali, i tipi di media e i confini di accettabilità esercitano anche influenza nel determinare se gli individui prendono parte alle discussioni politiche o meno. Per esempio, abbiamo trovato che nei nostri siti in Cina la repressione diretta della politica individuale era assente e forse persino non necessaria. Più importante si è rivelato lo sviluppo storico dei social media che significava che questi siti erano naturalmente visti come posti per l'intrattenimento e l'amicizia; l'impegno politico sarebbe apparso non adatto ai social media.

Nella nostra terza sezione l'enfasi era posta più su fattori locali: il modo in cui la politica tende a essere vista nel contesto dello spettacolo a Trinidad o come un mezzo per creare identità locale in opposizione alla politica nazionale, nel nostro sito in Cile. In un altro caso, per esempio il sito inglese, le persone semplicemente sentono più utile sfruttare la politica come una fonte di humor e non impegnarsi seriamente in essa. Ciò ci porta indietro al nostro punto iniziale e più ampio: i social media sono più spesso una modalità dell'interazione di gruppo su piccola scala e della socialità. Per la maggior parte della gente, per questo, ha più senso sfruttare la politica per rilanciare i social media piuttosto che usare i social per "fare" politica. 


\section{Capitolo 11 Immagini visive}

Angela ha 23 anni e vive nel campo di ricerca di Trinidad. Suo padre possiede un negozio di forniture per uffici dove lei lavora la maggior parte del giorno. Durante le pause lei fa scorrere la sua timeline di Facebook sul suo BlackBerry. Posta anche due o tre volte al giorno, iniziando con un saluto del tipo “Good morning peeps!” (“buongiorno, gente!”). A volte fa un'apertura su ciò che sta accadendo nella sua vita: "Anche se sorrido ancora, la gente non sa quanto può farti male". I post che attirano la maggior parte dei "mi piace" e dei commenti, all'incirca 20 "mi piace", sono però quel tipo di memi che Sinanan definisce "ispirati": versi religiosi o motti motivazionali con uno sfondo scenografico o animato. Quando Angela è annoiata e si dedica ai capelli e al trucco e posta un selfie, può attirare più di un centinaio di "mi piace". Dalla prospettiva dei suoi amici, i post visivi di Angela sono di gran lunga quelli più comunicativi.

In molti dei nostri siti, sui social media i post in modo preponderante sono visivi. La crescente popolarità di piattaforme come Instagram e Snapchat dimostra che i social media possono funzionare davvero quando il contenuto centrale è fotografico e il testo è relativamente periferico o quando, come in Snapchat (l'indizio è nel nome), ciò che siamo stati abituati a pensare come una conversazione può essere quasi interamente basata sul visivo ${ }^{1}$. È anche possibile popolare WhatsApp con fotografie di bambini e Facebook con selfie e memi.

Gli aspetti visivi dei social media sono stati un argomento costante per tutti i capitoli precedenti. Nei capitoli sul genere e sulla politica ci aiutavano a spiegare perché i social media visibili al pubblico sono in generale molto conservatori. Abbiamo anche visto l'uso di immagini fotografiche nei social media in relazione alla collocazione di classe e alle ambizioni sociali. Nei nostri siti in Brasile e a Trinidad, la classe sociale viene comunemente rivendicata visivamente grazie all'associazione con beni firmati. Un giovane può postare immagini di se stesso con bottiglie di liquori costosi e occhiali da sole di stilisti. Il suo pubblico tuttavia non necessariamente accetta questo come una dimostrazione di concreta ricchezza o status. I partecipanti alla ricerca in genere presumono che le persone tentano di mostrare ai loro pari le versioni migliori o idealizzate di se stesse, almeno sulle piattaforme più pubbliche. 
Ci sono diversi passaggi nella nostra analisi. Possiamo iniziare esplorando il modo in cui una persona cerca di usare associazioni visive per influenzare gli altri nella decisione di chi potrebbero essere, e in particolare quanto socialmente "esclusivi". Dobbiamo poi prendere in considerazione come gli altri rispondono a ciò e, soprattutto, come tali azioni rispondono a norme che riguardano il modo in cui, in quella società, è considerato appropriato o meno presentarsi, ossia ciò che definiamo comportamento normativo. Dato che questo è uno studio comparativo, a sua volta l'analisi metterà in luce in che modo ciò che è considerato normativo in una società si può comparare con ciò che lo è in altre. Tutto questo sarebbe vero per l'analisi visuale a prescindere dai social media, ma qui potrebbero anche esserci cambiamenti e elementi inediti. Per esempio, vedremo, in particolare, come i memi ora chiariscono bene non solo ciò che si considera normativo, ma anche come le persone ambiscono a controllare la dimensione normativa nei mondi online.

Per certi aspetti, tuttavia, la situazione in cui ci siamo imbattuti in questo progetto è stata profondamente diversa rispetto all'ambito dell'antropologia visiva tradizionale. Mentre nel passato gli antropologi avrebbero potuto dare la macchina fotografica alle persone locali per studiare come l'avrebbero usata, da circa un decennio le fotocamere dei telefoni sono oggetti comuni all'interno della maggior parte dei nostri siti. Le persone oggi sono decisamente aduse a fotografare e mostrare ciò che per loro è importante, anche oggetti che in precedenza probabilmente non erano così importanti da meritare una fotografia. I social media, così, sono molto di più che il semplice post di una propria foto. Le persone sanno come rielaborarle, come usare filtri su Instagram, come aggiungere testi personali a corredo e condivisione, come rimettere in circolo e ricostruire le immagini che costituiscono esse stesse dei memi.

I social media hanno dato alla fotografia una ubiquità senza precedenti, come parte della vita quotidiana. Mentre, un tempo, farsi scattare una fotografia rappresentava una sorta di piccolo evento, oggi dozzine di immagini possono fluttuare in pochi minuti proprio come lo svolgersi di una conversazione. Per di più, con la diffusione degli smartphone un'ampia popolazione di famiglie a basso reddito di tutto il mondo è ora diventata capace di fare fotografie sempre. Iniziamo con due affermazioni essenziali. La prima è che la stragrande maggioranza di tutta la fotografia oggi è fotografia sui social media. La seconda è che la nostra relazione con l'immagine visiva ha raggiunto un livello di ubiquità storicamente senza precedenti. Mentre mettiamo l'accento sulla fotografia, più di recente stiamo assistendo a un'altra rapida espansione 
di video clip brevi, postati anche su WhatsApp e condivisi su YouTube.

In ambito antropologico si è sviluppata una certa varietà di approcci all'immagine visiva e alla fotografia. Molti di questi riguardano la relazione fra le immagini, in quanto forma di rappresentazione, e le persone che le hanno prodotte. Esempi del modo in cui gli antropologi hanno studiato la fotografia includono Ginsburg, MacDougall, Sprague e, per delle rassegne ampie, Edwards e Pinney ${ }^{2}$. L'antropologia visiva è anche stata a lungo attenta ai sistemi visivi di particolari società ${ }^{3}$.

Altri approcci evidenziano il ruolo dell'etnografo nel rappresentare la società in cui lavora attraverso i film o la fotografia ${ }^{4}$. Nell'ambito di un progetto basato su nove siti di ricerca, dobbiamo essere attenti al tema della differenza culturale esattamente tanto quanto al modo in cui le immagini si collegano alle relazioni sociali in generale, anche se il nostro obiettivo principale è quello di definire il ruolo della componente visiva nei social media. Questo capitolo inizia con una breve discussione su come, in antropologia, generalmente è stato trattato il materiale visivo per mettere a fuoco, poi, la questione più specifica dei social media visivi. La tematica include l'auto-presentazione, la crescente accessibilità della comunicazione e il ruolo del visivo nel creare la socialità modulabile. Questi temi sono esplorati anche nel Capitolo 3 delle nostre rispettive monografie e nel volume dedicato alla comparazione del postare visivo in Inghilterra e a Trinidad ${ }^{5}$. Una delle questioni poste da questo volume comparativo riguarda il tipo di etnografia che emergerebbe se il focus fosse posto quasi interamente su ciò che impariamo da questo postare visivo, facendo un confronto con quanto sappiamo dalla più convenzionale ricerca etnografica offline. Il punto è che la nostra attuale capacità di vedere migliaia di immagini della vita quotidiana rende l'analisi visuale una parte estremamente significativa dell'inchiesta etnografica.

\section{Auto-presentazione}

Anche se esiste una percezione diffusa del fatto che l'auto presentazione online ha creato una relazione con l'espressione del Sé più artificiale o del tutto costruita, come Sherry Turkle ${ }^{6}$ indica nei sui primi scritti, gli esseri umani hanno di fatto sempre vissuto all'interno di ruoli e frame. Possiamo considerare il postare online come qualcosa che ha a che fare con la volontà di mostrarsi in pubblico in un certo modo, ma è importante notare anche 
che, almeno da Ervin Goffman in poi, gli studiosi hanno compreso che gli individui sono sempre impegnati in un processo di costruzione di se stessi, mettendosi in scena e presentandosi, consapevolmente, in certi modi. Questo, per esempio, è ovvio nello studio dell'abbigliamento. È del tutto possibile anzi che alcune persone ritengano di poter apparire più naturali online, all'interno dei loro gruppi di pari, indipendentemente da determinate interazioni offline che li rendono più consapevoli di loro stessi (per esempio, nell'incontro con un antropologo).

Il selfie è spesso criticato in quanto forma di mero narcisismo ${ }^{7}$. Di fatto i selfie possono forse essere visti come un genere importante per comprendere meglio temi come l'identità, l'aspirazione, le aspettative sociali. Certo, nello scattare selfie gli individui manipolano attivamente le impressioni che sperano di trasmettere, rendendo questo tipo di immagini una forma significativa dell'espressione del sé ${ }^{8}$. Il termine narcisismo, tuttavia, suggerisce un orientamento verso il sé laddove i selfie sono per lo più usati in relazione a pubblici specifici e per mantenere relazioni sociali ${ }^{9}$. Per esempio, nel nostro sito inglese i giovani postavano su Facebook cinque volte di più selfie di gruppo di quanto non facessero con immagini individuali. Inoltre, in quanto elemento interno ai social media, i selfie, insieme ad altre foto, hanno a che fare molto di più con azioni di condivisione e circolazione. Sia in termini di contenuto sia per quello che sono, i selfie rappresentano probabilmente un'attività focalizzata più socialmente che individualmente rispetto alla fotografia tradizionale - quasi l'opposto di ciò che si afferma comunemente ${ }^{10}$. Questa circolazione di immagini rafforza la condivisone di esperienze correnti e di ricordi ${ }^{11}$.

Nel confrontare il materiale presentato nel terzo capitolo di ciascuna delle nostre monografie, abbiamo capito che, mentre è banale rilevare che le persone forse vogliono creare versioni "idealizzate" di se stesse attraverso le immagini che postano, queste sono in relazione con contesti storici e sociali particolari ${ }^{12}$. In particolare, "aspirazione" e "sé idealizzati" sembrano significare cose molto diverse nei vari siti. Nella Cina industriale dominano post che esprimono l'aspirazione al consumo, fatto che non sorprende dal momento che si tratta di una popolazione di operai migranti che vedono il lavoro come una tappa per il raggiungimento della ricchezza. Fantasie di consumo di uomini giovani riguardano post con immagini di automobili, belle donne e vestiti firmati, mentre le donne postano stanze da letto da principesse. Soprattutto i giovani, postando queste immagini mostrano un mondo fantastico in contrasto con 
esistenze spese a lavorare in fabbrica. Grazie alle immagini visive, inoltre, possono comunicare emozioni, mentre non si sentirebbero particolarmente sicuri o eloquenti se postassero testi o se parlassero.

(a)
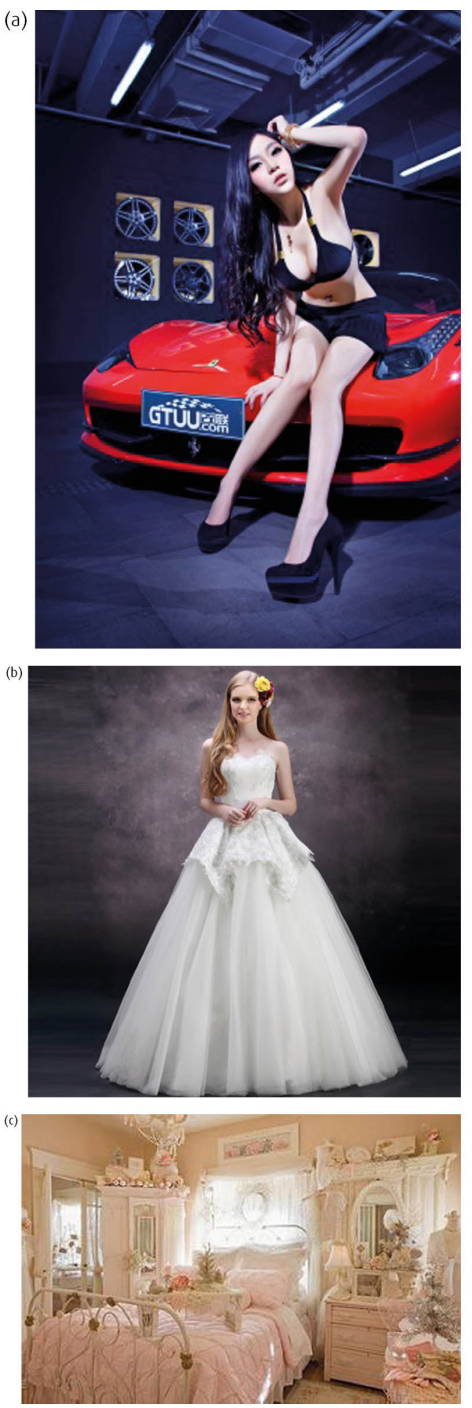

Fig. 11.1 Immagini di fantasie di consumo postate su $Q Q$ 
Viceversa, nel nostro sito nella Cina rurale, le aspirazioni economiche si intrecciano con le tradizioni conservatrici. Dominano post di bambini in momenti significativi, di cui fa parte la tradizione per cui i genitori spendono considerevoli somme di denaro negli studi fotografici. Queste foto, specialmente quelle scattate dopo i primi cento giorni del bambino, fanno riferimento anche a un debito del bambino verso i suoi genitori e all'obbligo che i figli devono avere verso i genitori più avanti nella vita. Accanto a post che riguardano i bambini, i post sull'amore e il matrimonio enfatizzano l'importanza di relazioni durature a fondamento della vita familiare, mentre altri esprimono affetto e riconoscenza, ostensivamente rivolti ai genitori. Benché il focus principale riguardi i valori tradizionali, la fotografia sui social media sta anche iniziando a esprimere le aspirazioni individuali, per una storia d'amore nella relazione di coppia, per esempio, o per l'autonomia dei giovani dai loro genitori.

(a)

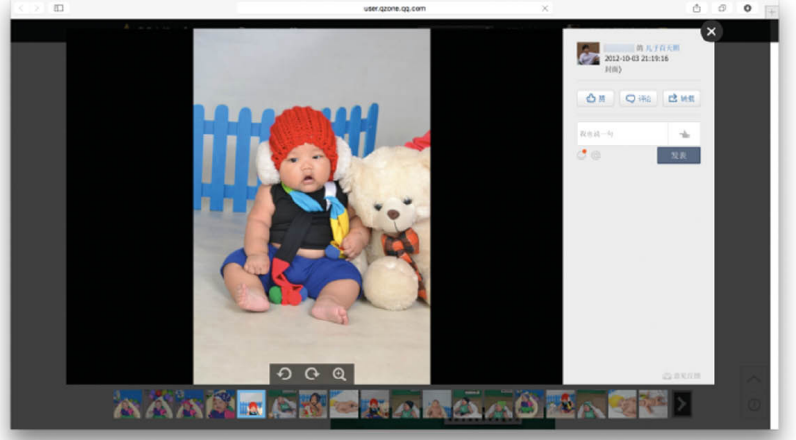

(b)

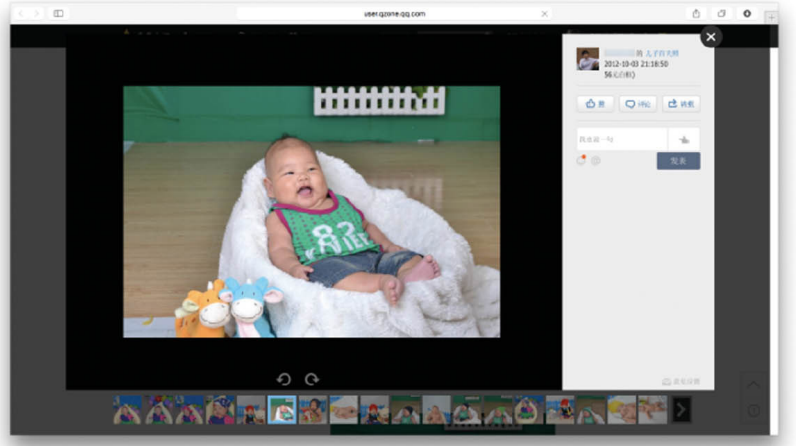

Fig. 11.2 Immagini di bambini scattate in uno studio professionale e postate dai genitori su $Q Q$ 
Nei casi del Brasile e di Trinidad, guadagnare più grande visibilità sociale è un'aspirazione in se stessa e appare soprattutto su Facebook. Nel sito brasiliano i social media si affiancano alla chiesa come spazio che conferisce visibilità a nuova ricchezza o all'aspirazione a essa. La storia di Sandra nel Capitolo 9 è in questo senso significativa. I Cristiani evangelici credono che la ricchezza ottenuta mediante il duro lavoro rappresenti una delle virtù pubbliche di una persona; di conseguenza abbracciano un materialismo visibile come segno di religiosità, non di superficialità. A Trinidad, le persone considerano il culto dell'apparenza esteriore come prova dell'effettivo sforzo che una persona compie piuttosto che come distorsione di un sé autentico e naturale. Le persone per questo si impegnano in modo considerevole per creare immagini che mostrino questo processo di costruzione della bellezza, e dunque l'idea della profondità dell'apparenza.
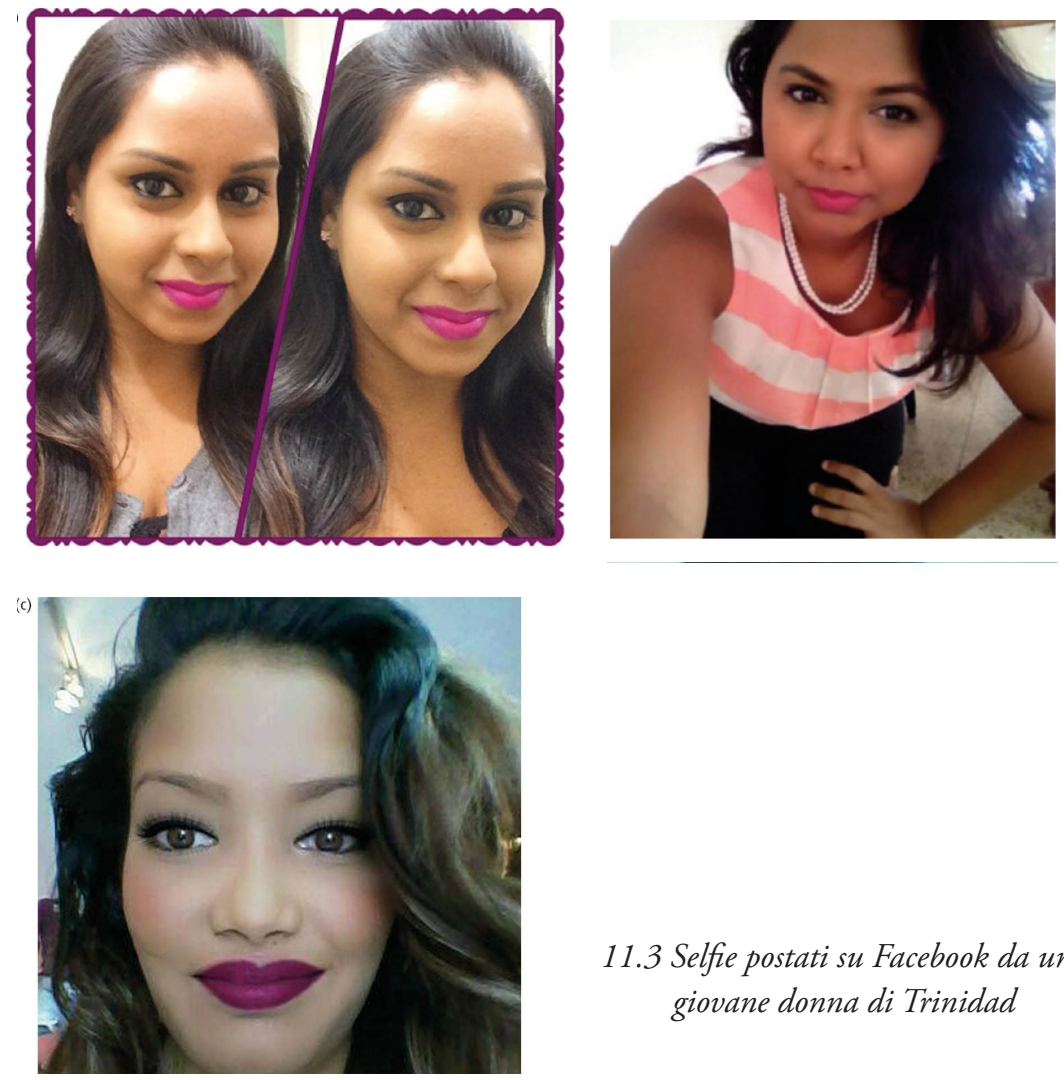

11.3 Selfie postati su Facebook da una giovane donna di Trinidad 
Nell'Italia del sud la visibilità di una persona sui social media pubblici è pesantemente intrecciata con la sua posizione sociale. Le persone percepite come più in alto nella gerarchia sociale locale postano più fotografie di se stesse di quanto non facciano quelle di livello inferiore. Nicolescu sostiene che questo fenomeno è collegato a più antiche tradizioni di visibilità della società italiana per chi fa parte delle classi più alte. Eppure, questa tradizione è posta in discussione dalle nuove tendenze di teenagers e giovani di postare selfie su Facebook. Le donne giovani trascorrono ore a provare un "buon" selfie. Scelgono dalle molteplici foto che hanno scattato, le editano online e infine ne caricano una come immagine del profilo. Questa passione per l'autoespressione online cala dopo il matrimonio, quando le italiane cominciano a postare meno di se stesse e di più della loro famiglia, dei traguardi dei figli e di riunioni fra diverse famiglie. [Fig. 11.3 a, b, c]

La cura di come si appare su Facebook era importante anche nel nostro sito del sud dell'India. Qui era visto in termini di costruzione di un'identità personale, spesso come parte di un'affermazione di status associata al proprio lavoro. Numerose persone postavano immagini di se stesse in tenuta da lavoro o in contesti evidentemente professionali, anche qualora il loro lavoro reale fosse qualcosa di completamente diverso da ciò che queste immagini visive implicassero. In tutti questi casi, quindi, possiamo affermare che le persone usano le immagini visive per esprimere aspirazioni, ma ora è evidente che la natura di queste aspirazioni è piuttosto locale e specifica.

In certi casi, le regole che normano l'auto-presentazione non riguardano l'apparire al proprio meglio, ma solo il presentare se stessi in linea con le aspettative sociali. Questo è emerso molto chiaramente nei contesti di ricerca del Cile settentrionale e della cittadina inglese; qui a colpire è la prevalenza, nelle immagini postate, della semplice ordinarietà. I selfie tipici nel Cile del nord, anche per i giovani, sono di solito scattati a casa loro o di amici, al lavoro o durante le pause; non trasmettono il senso di un gran fascino. Teenager e giovani spesso postano molte immagini al giorno, di solito su soggetti banali come le nuove scarpe da ginnastica, la colazione, l'auto appena lavata, selfie scattati a scuola o al lavoro e collage di foto fatti con un'altra app, per trasmettere il senso della monotonia quotidiana della vita e spesso con hashtag come \#annoiato \#aburrido o \#fome ${ }^{13}$. Poche fotografie esprimono persino il massimo della noia: attendere in coda mentre si sbrigano commissioni.

Una versione comune del selfie è la foto dei piedi, o "footie", quasi sempre 
scattata in posizione distesa mentre si guarda la televisione o si gioca a un video game. Non solo questo dà all'osservatore il senso della vita quotidiana che essi vogliono cogliere, ma è anche una dimostrazione del fatto che semplicemente non serve mettersi in posa. La "footie" è così casuale che il fotografo non deve nemmeno muoversi dalla sua posizione di riposo.

(a)

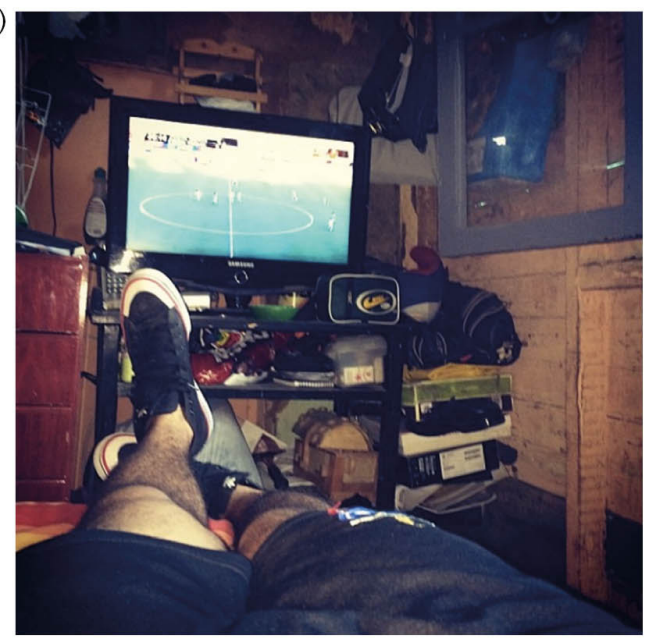

(b)

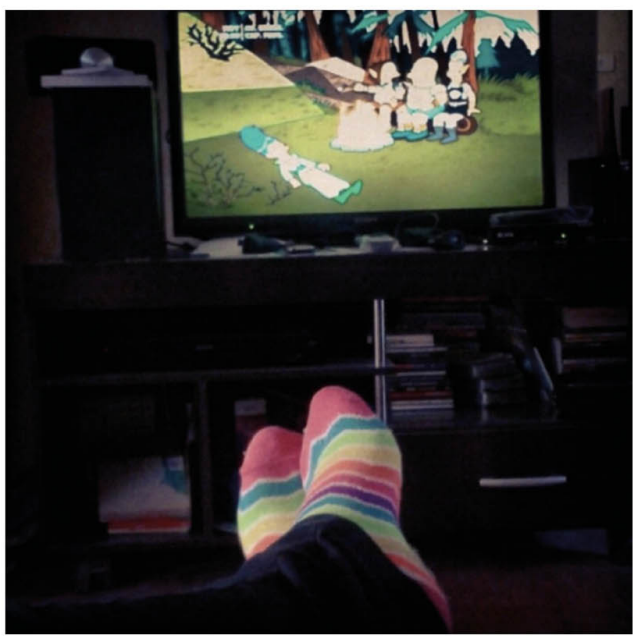

Fig. 11.4 "Footies" postati da giovani cileni 
Nell'Italia del sud appare lo stesso genere, ma quasi sempre preso sulla costa o con i piedi rivolti verso il mare. Qui il footie simbolizza la presenza dell'individuo immerso nello spettacolo e nella bellezza della natura. La stragrande maggioranza dei post fatti da adulti nella cittadina inglese sembra mostrare l'assenza di qualsiasi tentativo di essersi vestiti o preparati per l'immagine. La fotografia sui social media sembra essere associata al declino del trucco e all'onnipresenza di vestiti come i jeans e le T-shirts, all'insegna di una modestia senza pretese. In effetti, gli adulti della cittadina cominciavano a postare selfie solo dopo che una causa apparentemente pia basata su post del tipo "no make-up selfie" ("selfie senza trucco") li aiutava a prendere le distanze dall'associazione prevalente 'selfie' e 'narcisismo'.

Qui si trovano elementi sia di continuità sia di cambiamento rispetto agli usi precedenti di questo tipo di fotografia. Nello suo studio sulla fotografia contadina in Francia negli anni Sessanta, Bourdieu notava che le fotografie scattate più di frequente riguardavano nozze e feste collettive, anche se queste immagini venivano custodite nelle scatole, e solo poche fotografie delle nozze erano esposte in casa ${ }^{14}$. Egli afferma che questi tipi di fotografie sono "sociogrammi", e sono una testimonianza visiva di ruoli e relazioni sociali. Queste cerimonie sono ritenute degne di essere fotografate perché esulano dalla quotidianità. Inoltre, rendono solenni e materializzano questi "picchi di vita sociale" allorché il gruppo riafferma la propria unità ${ }^{15}$.

Viceversa, molta della fotografia sui social media è oggi banale, e su siti come WhatsApp ha a che fare più con ciò che passa piuttosto che con la memoria. Si possono tuttavia trovare anche esempi di continuità, per esempio quando Facebook è usato oggi come il luogo in cui registrare in qualche modo occasioni speciali o celebrazioni. La fotografia oggi è anche associata con i biglietti d'auguri. Le persone riconoscono relazioni inviando e condividendo foto, ma anche memi nello stile di biglietti d'auguri in occasioni speciali, in particolare a Trinidad e nel sud dell'India. Diwali, Natale, Capodanno, compleanni e lauree sono tutti eventi per i quali tradizionalmente, a Trinidad, si può inviare un biglietto; queste occasioni oggi spesso sono marcate facendo circolare immagini su Facebook. 
(a)

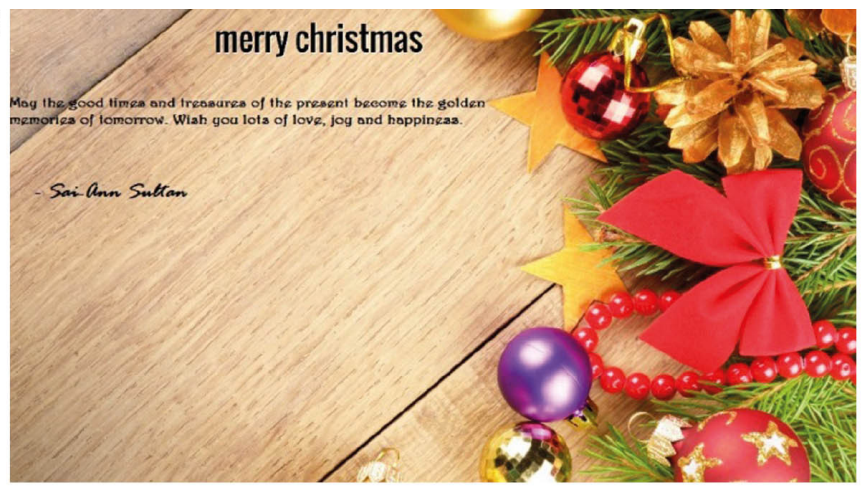

(b)

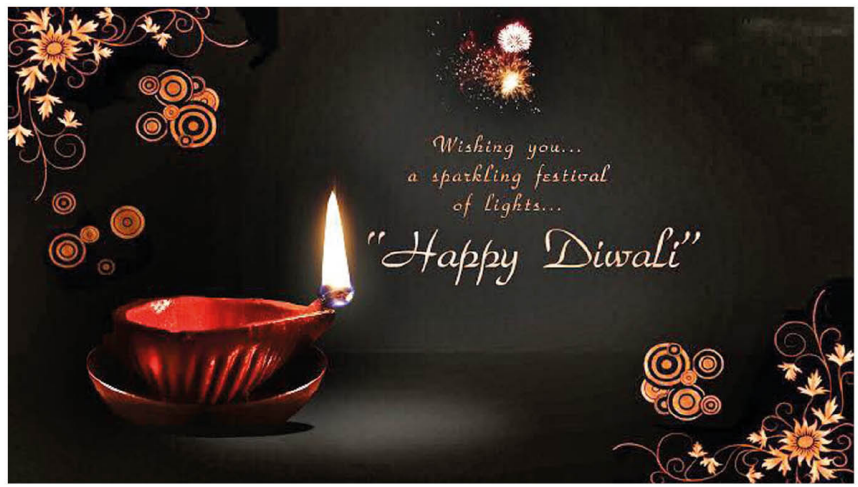

Fig. 11.5 Biglietti di auguri condivisi dagli abitanti di Trinidad su Facebook

Nel nostro sito nel sud dell'India, oltre alle occasioni speciali, vengono postati saluti quotidiani quasi ritualisticamente, per la comune credenza, basata sul concetto hindu di karma, che la positività deve essere condivisa con una persona della propria rete di relazioni per bilanciare la negatività che c'è intorno. Questi post sono di solito parole su uno sfondo semplice o con un'immagine scenografica, accompagnate da un titolo che recita "Good morning", "Have a grt...dy" o "Have a good day". 
Come il mondo ha cambiato i social media

(a) Gud afternoon friends - with

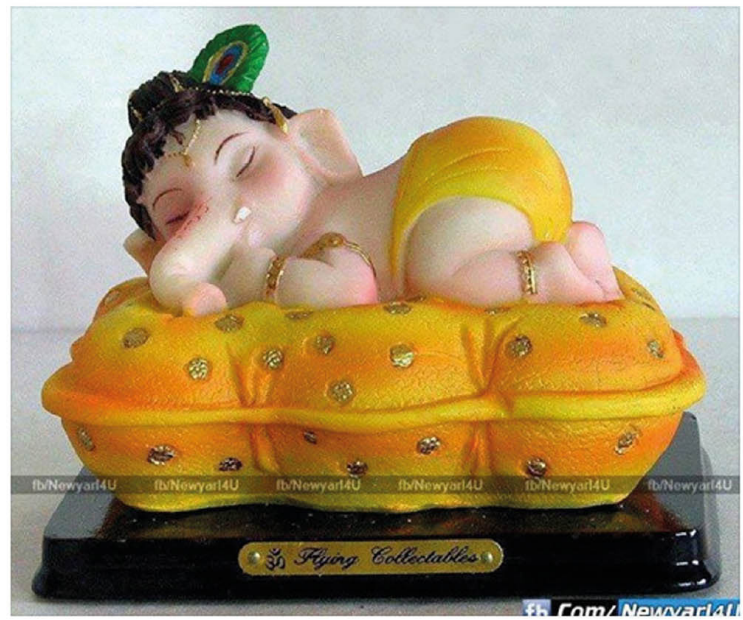

Share

1 share

(b) Gud even.

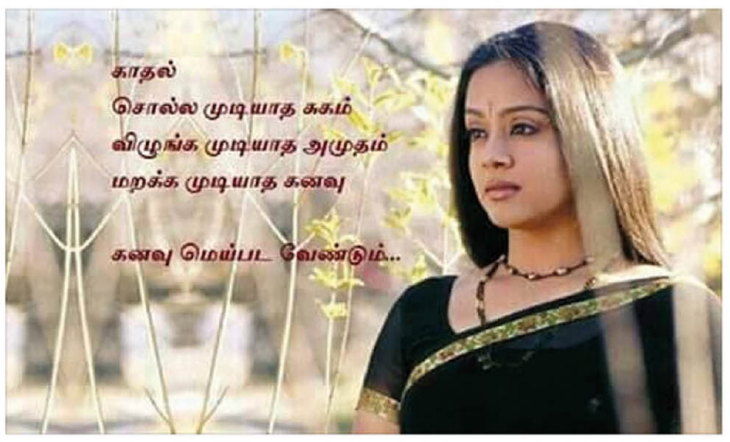

$$
\text { It Like Comment }
$$

20 people like this.

Write a comment.

이 (ㄷ)

Press Enter to post.

Fig. 11.6 Saluti pomeridiani e serali circolati su Facebook nel Tamil Nadu 
I social media non rappresentano soltanto un passaggio verso l'onnipresenza delle immagini visive. In alcuni casi questo emerge dall'uso di postare giornalmente fino a centinaia di fotografie che, in precedenza, erano rigorosamente tenute fuori dallo sguardo pubblico. Questo rappresenta probabilmente uno dei cambiamenti principali non solo nella fotografia personale, ma anche nel modo in cui le persone intendono le relazioni sociali. Lo vediamo nel nostro sito nella Turchia sudorientale. Qui, prima dei social media, le fotografie erano un fatto privato, eccezion fatta per i ritratti formali o per le foto di nozze, esposti in casa o conservati con cura negli album. Le immagini tenute nelle scatole erano più informali e ritraevano membri della famiglia vestiti in modo più casual, e circolavano solo nell'ambito familiare, in poche occasioni limitate e controllate. Con Facebook, tuttavia, gli eventi familiari quotidiani come pranzi e cene sono diventati importanti eventi visibili. Eppure, a causa di limiti e convenzioni locali di rendere pubbliche fotografie di persone, molti utenti di Facebook hanno iniziato a trovare modi per esprimere lo stesso il loro attaccamento ad altre persone e la qualità del tempo trascorso insieme, ma senza mettere troppo a fuoco il loro viso. Quindi alle riunioni familiari si preferisce fotografare i diversi piatti serviti durante la cena e le tavolate preparate prima. In questo modo, essi rispondono alle nuove possibilità di circolazione, ma, tenendo conto degli aspetti tradizionali della privacy, hanno preso l'accorgimento di abbassare lo sguardo dal volto al cibo.

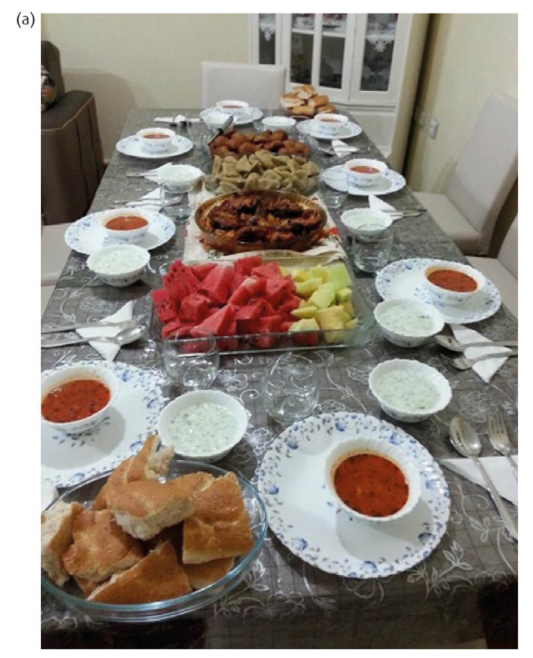



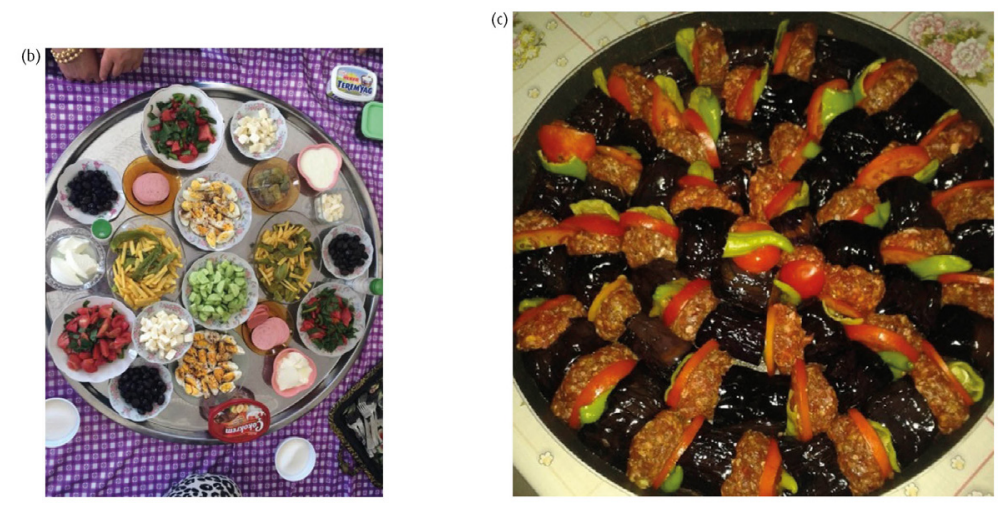

Fig. 11.7 Immagini di cibo scattate in occasione di riunioni familiari nel sudest della Turchia.

\section{Comunicazione sempre più accessibile e morale}

Un risultato abbastanza netto della comunicazione online diventata più visuale riguarda il fatto che essa è anche più accessibile per chi ha un basso livello di alfabetizzazione. Proprio come i fumetti invitavano coloro con scarsa istruzione a leggere di più, gli aspetti visuali dei social media incoraggiano gruppi analoghi a farsi più attivi digitalmente ${ }^{16}$. Nel sito in Brasile, sui social media gli adulti volevano essere attivi proprio come gran parte della popolazione più giovane. Ciò non tanto perché volessero mantenersi al passo con le tendenze giovanili, quanto piuttosto perché essere presenti sui social media era un modo per mostrare se stessi come più aperti all'esterno e più mondani, in possesso di risorse sufficienti per acquistare uno smartphone e competenti e a proprio agio con le nuove tecnologie, tutte cose che connotano "l'essere moderni". Nell'ultimo ventennio, in Brasile il livello di alfabetizzazione tra i più poveri è rapidamente cambiato. Circa un terzo della popolazione sopra i 50 anni è analfabeta, mentre tra i teenager lo è solo il due per cento ${ }^{17}$. Mentre i più giovani probabilmente vedono i social media come lo spazio dove costruire la propria immagine personale al riparo dallo sguardo degli adulti, sempre di più anche gli adulti desiderano semplicemente socializzare nello stesso spazio, con i loro coetanei. 
Gli adulti con un basso livello di alfabetizzazione usano Facebook, più per far circolare contenuti che per postarne di loro. Il "mi piace" era l'attività più comune, benché le persone facessero anche commenti più brevi, in forma abbreviata, tipo "kkkkk", l'equivalente di "lol" ("lots of laughs", "un sacco di risate"). Anche se questo gruppo non posta così tanti stati personali, attraverso memi, immagini e video condivisi può continuare a esprimere la sua opinione, i suoi valori e le sue preferenze. I memi, pertanto, diventano più importanti in quanto modo di esprimersi per coloro che sono meno capaci di esprimersi e meno a loro agio con le parole in uno spazio pubblico.

(a)

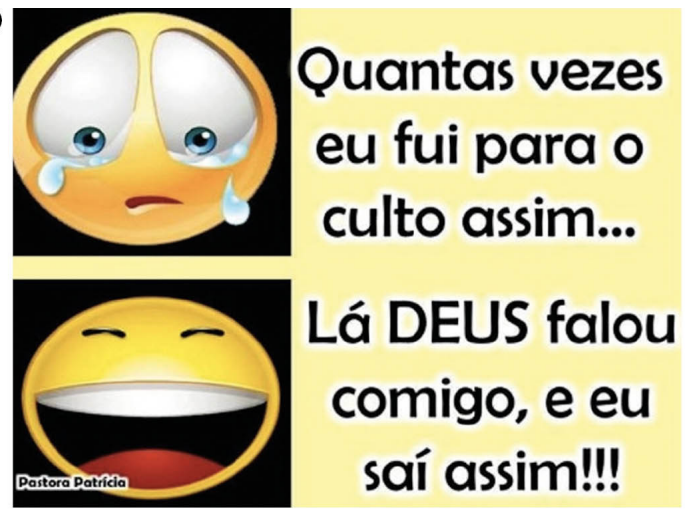

(b)

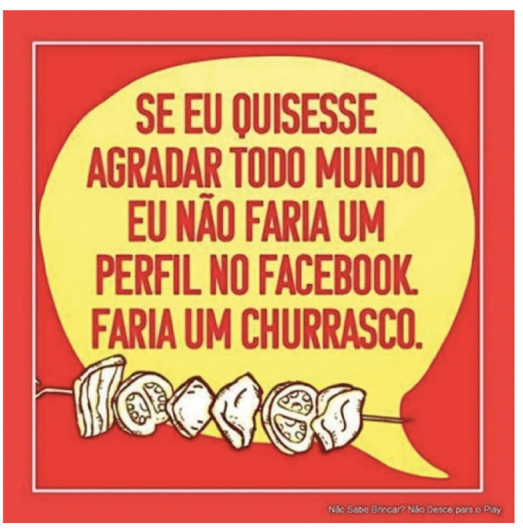

Fig. 11.8 Memi circolati su Facebook nel nordest del Brasile: "Sono andato in chiesa tante volte cosi... poi Dio mi ha parlato e ora mi sento cosi!" e "Se avessi voluto far contenti tutti non avrei fatto un profilo su Facebook, avrei fatto un barbeque" 
Nel sito dell'India meridionale, i simboli di Facebook, per esempio pollici in alto per "mi piace" e la freccia per condividere, consentono di partecipare anche ai giovani con basso livello di alfabetizzazione. Come nel nostro sito brasiliano, le condivisioni riguardavano per lo più immagini, memi e video clip. Il semplice fatto di essere in grado di mettere "mi piace" ai post di altri rende questi utenti più a loro agio nell'essere socialmente presenti online. Anche se il livello di alfabetizzazione nel sito della Cina industriale era più elevato, anche diversi gruppi poco istruiti usavano il visivo sui social media come forma di espressione. Per quanto riguarda la popolazione rurale migrante nella città industriale, soprattutto i giovani condividevano immagini editate da altri con testualità motivazionale e emotiva e immaginario pittoresco. Alcuni accompagnavano queste immagini condivise con poche parole personali, come "wo" (Io), intendendo che l'immagine esprime ciò che la persona pensa o sta provando.

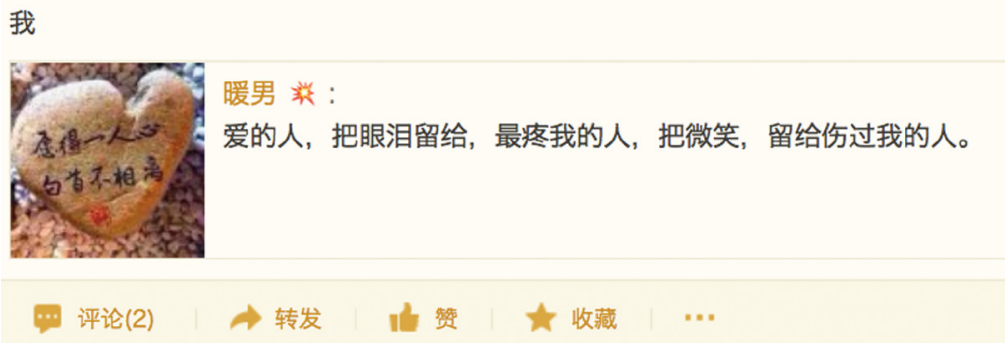

Fig. 11.9 Questo post su QQ recita: "lascerò le mie lacrime a coloro che mi hanno davvero amato, e lascerò un sorriso a coloro che in passato mi hanno ferito". La persona che lo ha condiviso ha commentato "wo" - "io" in mandarino - in cima al post.

Per popolazioni più istruite le immagini servono a espandere l'ambito degli argomenti e delle esperienze comunicate. Il genere più popolare di memi nell'Italia meridionale riguarda idee e ideali più astratti che le persone non necessariamente avrebbero voglia di articolare in un testo, per esempio quando criticano la politica. Più in generale, i memi creano un modo indiretto di riflettere sulla società che non viola le norme sociali, al contrario di un post verbale che vincola le persone alla loro posizione sociale.

I memi circolano come una modalità per moralizzare e per fare umorismo; in questo senso, sono un modo per rafforzare le norme sociali. Nel nord del Cile, i memi spesso rappresentavano l'accettazione della vita quotidiana o an- 
che il fatto di esserne orgogliosi. Esempi chiave riguardavano il genere 'Kermit la Rana' ("la Rana Rene" in spagnolo), per l'espressione di sentimenti tipo "A volte vorrei avere una casa davvero grande. Poi mi ricordo che non mi piace spazzare e non ci penso più". Altri mettono in contrasto le "aspettative", foto di corpi in forma, abbronzati, auto nuove fiammanti o cibo di classe, con la "realtà" di corpi sovrappeso, veicoli arrugginiti e una cena bruciata.

(a)

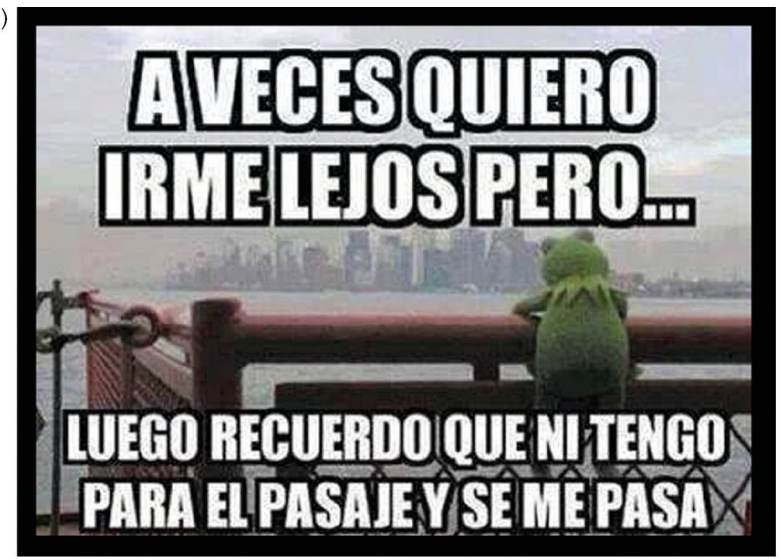

(b)
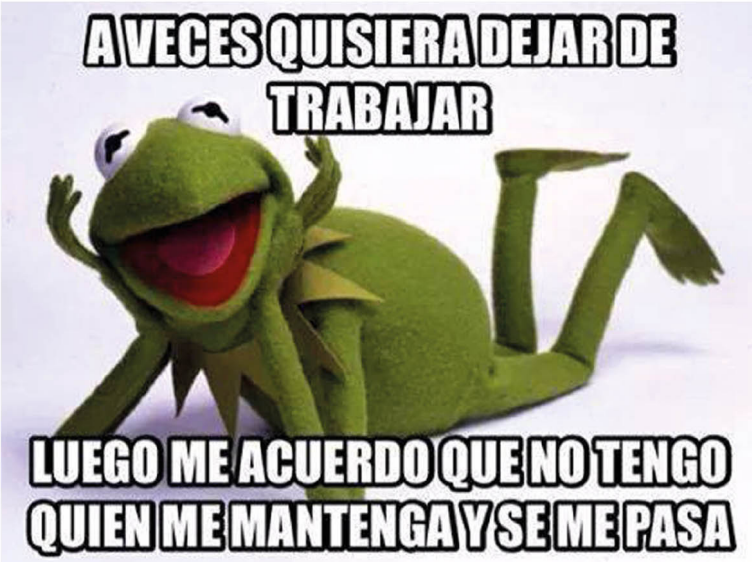

Fig. 11.10 Memi di Kermit postati su Facebook nel sito in Cile: "A volte vorrei andarmene lontano, ma poi mi ricordo che non ho abbastanza nemmeno per coprire il costo di un biglietto e non ci penso più" e "A volte vorrei licenziarmi. Poi mi ricordo che non ho nessuno che mi mantenga e non ci penso piü". 
Scherzare consente alle persone, secondo una modalità sicura e comune, di esprimere le loro preferenze per lo stile di vita con cui si sentono a loro agio, così come di criticare quelli che li guardano dall'alto in basso ${ }^{18}$. Trinidad mostrava la sua specifica declinazione del meme Kermit, ispirata a una pubblicità televisiva del Tè Lipton ${ }^{19}$. Nella pubblicità, Kermit è seduto in un bar e osserva da lì il caos di New York. Sorseggia con calma il suo Tè e pronuncia la frase a effetto: "Ma non sono fatti miei". Nei memi che apparivano nel sito di Trinidad, la frase a effetto era usata per far notare che cosa stava facendo un'altra persona o un altro gruppo. Sotto questa forma umoristica possono circolare commenti critici molto seri. Dato il comune uso dei memi di questo tipo in tutti i nostri siti, sembra rilevante considerarli più in generale come una forma di "polizia in rete", un tentativo di affermare un controllo morale attraverso i social media.

\section{(a) You Be Lurking On My Page}

Cause You Miss Me But Thats

None Of My Business...

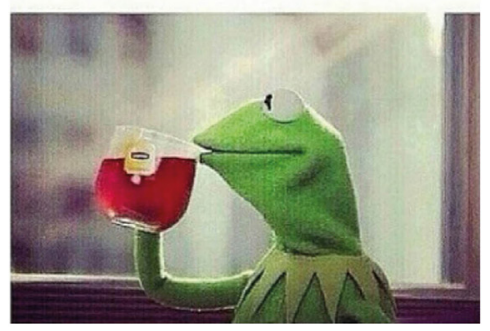

b) dudes be like "these hoes ain't loyal" but have a wifey, 4 side chicks and 2 "friends"... but that's none of my business

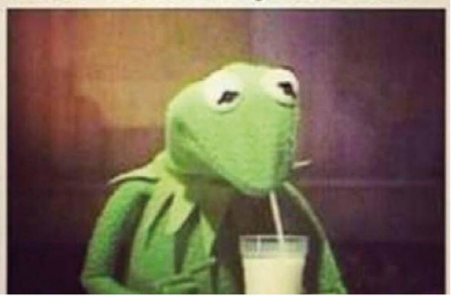

Fig. 11.11 Memi di Kermit che circolavano su Facebook a Trinidad 


\section{Socialità modulabile}

Ci sono dei precedenti rispetto al modo in cui sui social media circolano immagini, fondati sulla preesistente diffusione di telefonini con macchina fotografica e, più in generale, di immagini digitali, studiati ampiamente nell'ambito di altre discipline quali HCI (Human Computer Interaction) e STS (Science and Technology Studies). Ito, che ha condotto uno studio etnografico sugli usi delle fotocamere telefoniche in Giappone, descrive questa condivisione pervasiva di foto come "stretta co-presenza visuale": una nuova forma di consapevolezza sociale e di scambio di percezioni e prospettive ${ }^{20}$. Suggerimenti simili sono stati fatti riguardo alla diffusione di fotocamere telefoniche e piattaforme di condivisione di foto più in generale ${ }^{21}$, anche con dei rimandi al tema precedente relativo a modo in cui queste vengono usate per mantenere le relazioni sociali ${ }^{22}$.

Lindtner et al. e altri hanno notato che le immagini visive sono condivise con una varietà di tipi diversi di "pubblico" 23 . Per esempio, quando gli amici vedono ciò che hanno postato amici dei loro amici allora il senso di "questo è fatto apposta perché io lo veda" va oltre la precedente relazione fra queste persone. Molte persone oggi hanno numerosi network che probabilmente prima erano distinti, per esempio amici, colleghi di lavoro e familiari, ora messi tutti insieme dentro lo spazio aperto di Facebook. Un modo in cui le persone affrontano la questione del "collasso del contesto" ${ }^{24}$ è postando immagini che hanno riferimenti comprensibili solo ad alcuni di quelli che le guardano e non ad altri. Come indicato nel Capitolo 1, la socialità modulabile può essere costruita in questo modo dentro una data piattaforma, e non solo mappando gruppi sociali diversi in piattaforme diverse.

Benché l'intenzione sia quella di confermare un in-group rispetto a un pubblico, sulla piattaforma restano comunque "quelli che sentono di sfuggita" ("overhearers"), coloro che non interagiscono con la persona che posta, che non sono il pubblico cui ci si riferisce, ma che attingono significati da ciò che vedono ${ }^{25}$. Quando le persone prendono parte a molteplici pubblici contemporaneamente, non deve sorprendere che possa esserci un problema di immagini considerate appropriate da alcuni e non da altri. Abbiamo notato che in ogni contesto le persone trovano il modo di far fronte a questo fatto. In alcuni casi, questo riguarda la socialità modulabile, per esempio a Trinidad, utilizzando contenuti incomprensibili per alcuni. Oppure, come nel nostro sito in Italia meridionale, molte persone scelgono di postare su 
piattaforme più ristrette, per esempio WhatsApp, cose che si possono ritenere inappropriate. Nel sito della Turchia sudorientale, invece della socialità modulabile abbiamo trovato che le persone tendono sempre più a postare foto di cibo invece che di persone a eventi, così che nessuno sia escluso. A volte, però, queste strategie volte a tenere ciò che si posta all'interno di uno specifico pubblico, falliscono e il collasso del contesto semplicemente espone immagini inadatte a pubblici inadatti. L'esempio più ovvio di esposizione non intenzionale riguarda la circolazione di immagini sessuali, in origine scattate e condivise come messaggi privati nel contesto di relazioni intime. Nell'India del sud c'è un fenomeno di fotografie indiscrete di donne prese dai loro telefoni cellulari. Le donne non postano queste immagini da sole, ma sono gli uomini a cui le inviano a tradire la loro fiducia caricandole online. Di conseguenza, le donne stanno diventando sempre più attente nell'accertarsi che i loro telefoni siano protetti e sempre vicini; si assicurano anche che i loro partner siano altrettanto accorti con i loro telefoni. Infine, le donne raramente postano fotografie di se stesse da sole, mentre posano per foto di gruppo e le caricano. Postano anche immagini con valore simbolico, per esempio l'eroina di un film o un'icona della cultura pop, utilizzate per rappresentare se stesse.

Il fenomeno noto come "revenge porn" ("porno vendetta") venne rilevato a Trinidad un po' di tempo prima che questa espressione fosse inventata per descrivere il dilagare della pubblicazione deliberata di materiali intimi dopo la fine di una relazione. Nelle scuole inglesi e altrove c'è stata una breve fase nello sviluppo di Snapchat durante la quale gli utenti non avevano ancora capito che le immagini potevano facilmente essere catturate dallo schermo. La conseguenza fu che alcune ragazze condivisero immagini di cui in seguito si pentirono.

Questa involontaria fuoriuscita di immagini non è limitata a contenuti sessuali e al nudo. Per esempio, problemi simili emergono quando un post diretto ai propri pari che si lamentano di un amico è poi visto dai colleghi di lavoro. La sola presenza di un'immagine di qualcuno dell'altro sesso può esacerbare timore e gelosia all'interno di una coppia sposata. Un altro scenario si presenta quando fotografie postate sono prese fuori contesto. Potrebbero ritrarre feste e celebrazioni dove le persone tendono a bere e comportarsi diversamente da come farebbero al lavoro o in famiglia; in queste circostanze è difficile controllare la percezione dell'immagine da parte del nuovo pubblico. 
Non si dovrebbe però presumere che la fuga di immagini sia sempre involontaria. Esiste un confine sfumato fra questo argomento e la nascita dei "messaggi indiretti". Per esempio, uno studente inglese potrebbe postare la foto di un maiale su una spiaggia intendendo fare riferimento a qualcuno della classe, ma senza dire chi. A Trinidad, la popolazione tende a vedere se stessa come particolarmente dedita al gossip e allo scandalo (il termine locale è "bacchanal"). Per questa ragione vi è un interesse considerevole per il modo in cui gli oggetti visuali forniscono "evidenza" per la giustapposizione fra persone che si presta a infinite interpretazioni ${ }^{26}$. Il postare visivo ambiguo è a volte incoraggiato come forma di intrattenimento popolare e come equivalente visivo dell'allusione. D'altra parte, il fatto che non si può mai sapere chi potrebbe scattare foto e postarle sui social media, a quanto pare, a Trinidad, ha reso l'infedeltà meno comune e, in Cina, ha ridotto la corruzione. Da questi cambiamenti possono scaturire conseguenze impreviste. Un'azienda che vende alcolici di alto livello in Cina ha dichiarato un crollo delle vendite per il fatto che ormai i funzionari temevano che sui social potessero apparire foto che li traevano mentre bevevano questi liquori costosi.

\section{Conclusioni}

Sottolineare molte delle discussioni di questo capitolo è un altro punto generale. I social media rappresentano una significativa accelerazione della possibilità che la comunicazione possa diventare più visiva, nel senso che è ora possibile tenere qualcosa di molto simile a una conversazione fatta quasi interamente senza voce o testo. Nel sito inglese, Snapchat è la piattaforma di social media dominante per molti teenager, ed è tipicamente usata in questo modo conversazionale. Le persone scattano fotografie del loro viso chiaramente esemplate sulle emoticon come mezzi semplici per trasmettere come ci si sente in un dato momento. [Fig. $11.12 \mathrm{a}$, b] Il fatto che qualcosa come 750 milioni di fotografie sono in circolazione ogni giorno su questa piattaforma significa che oggi si tratta di un contributo rilevante all'uso delle immagini visive nella comunicazione. 
(a)
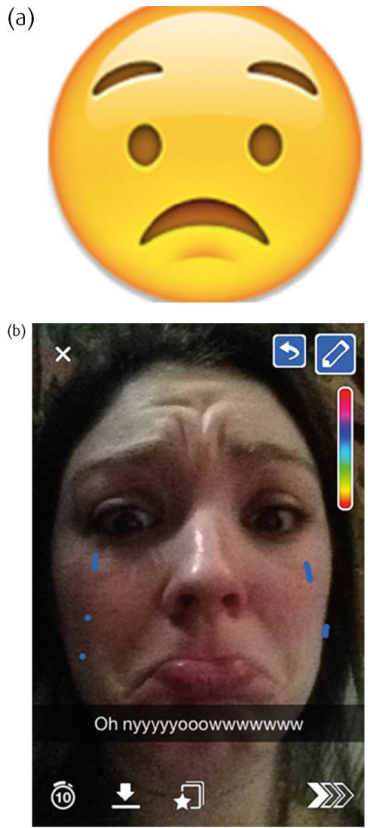

(c)

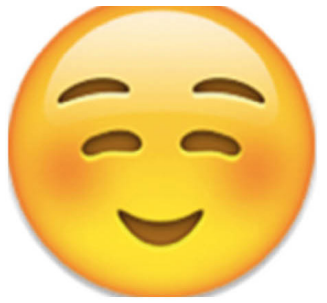

(d)

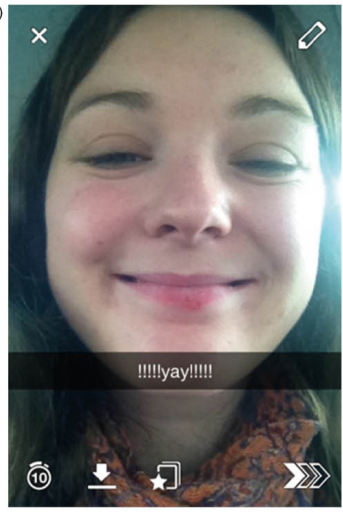

Fig. 11.12 Immagini che mostrano come l'espressione facciale possa emulare quelle delle emoticon su Snapchat.

Questo è solo un esempio di una più ampia crescita di nuovi modi per mezzo dei quali si possa dire di più con le immagini, messi a punto dagli utenti dei social media e da coloro che sviluppano queste tecnologie. Altri esempi riguardano il potenziale di espansione legato al fatto che persone con un basso livello di alfabetizzazione hanno oggi la possibilità di partecipare ai social media. Un altro consiste nella capacità delle persone che hanno una scarsa fiducia in se stesse di trovare modi nuovi di esprimere sentimenti in modo indiretto attraverso i memi. Un terzo riguarda lo sviluppo del meme come un mezzo potente, anche se indiretto, di sorvegliare la "moralità" di internet.

Niente di tutto ciò significa necessariamente che ciò che viene comunicato sia nuovo. Come abbiamo sostenuto in altri capitoli, per esempio quello sul genere, la crescita in visibilità creata dai social media ha spesso portato a un maggiore conservatorismo. Questo si è verificato in special modo nel nostro sito nel sudest della Turchia. Abbiamo visto anche come la fotografia venga usata per rafforzare valori familiari tradizionali nella Cina rurale. Eppure, 
allo stesso tempo, troviamo conseguenze del tutto diverse nel nostro sito della Cina industriale, più orientato alla fantasia e al futuro piuttosto che alla tradizione e al presente ${ }^{27}$. I post visuali dimostrano che le persone usano i social media per reinventare la loro personale idea di tradizione, conformismo e normatività, motivo per cui la comprensione esatta del contesto è essenziale per cogliere ciò che le persone scelgono di postare.

Questo capitolo ha pure dimostrato che la socialità modulabile riguarda anche la gestione multipla di pubblici diversi all'interno della medesima piattaforma, e non solo fra più piattaforme. La combinazione indica che navigare tra questi diversi tipi di pubblico all'interno del proprio social network è un problema crescente. Uno stesso social medium potrebbe essere usato per creare gruppi piccoli e separati, con particolari interessi, ma anche per rivendicare e creare un ampio consenso attorno a questioni morali o, semplicemente, per pratiche quotidiane come l'espressione di saluti giornalieri nel sud dell'India o il rifiuto del glamour nei selfi degli adulti nel nostro sito inglese e per i 'footie' cileni. Tutto ciò può essere usato per costruire, sui social media, una banale "estetica ordinaria" della onnipresente fotografia ${ }^{28}$. Oggi, come nel passato, la questione della rappresentazione e della comunicazione è più complessa per il fatto che una foto è considerata una sorta di 'prova', cosa che implica ulteriori questioni di affidabilità e verità ${ }^{29}$.

Questo potrebbe essere il punto di interesse antropologico finale. Con le nostre etnografie cerchiamo di seguire le dinamiche delle norme culturali delle popolazioni che studiamo. Possiamo, letteralmente, vedere se la comprensione del genere che riguarda uomini uniformemente rappresentati come lavoratori manuali e donne come lavoratrici di cura è in aumento o in diminuzione. Questo enorme aumento di immagini visive, insieme al consistente numero di foto e al carattere morale dei memi oramai abbastanza esplicito, può assisterci in questa impresa. Per cercare di capire i valori delle persone abbiamo tratto un grande vantaggio dalla nostra capacità di esaminare migliaia di post visuali che fanno parte della comunicazione quotidiana. $\mathrm{Ci}$ troviamo a un vero e proprio stadio iniziale, ma è probabile che se nella comunicazione testuale e orale le immagini visive stanno diventando sempre più un importante complemento, allora queste costituiranno anche una componente sempre più importante dell'antropologia ${ }^{30}$. 



\section{Capitolo 12 \\ Individualismo'}

\section{Individualismo e network}

Al centro del pensiero occidentale c'è stata, almeno a partire dal Romanticismo ${ }^{2}$, una particolare attenzione per la natura dell'individualismo, in equilibrio tra il timore che potesse crescere a discapito del nostro impegno sociale, e il desiderio di alimentarlo in quanto progetto esistenziale. Pressoché tutti i fondatori delle scienze sociali si collocano nella grande narrazione che dà per scontato nel mondo occidentale un movimento complessivo verso l'individualismo ${ }^{3}$. In modi diversi questa include Mauss, Marx, Simmel, Tonnies e Weber. Questa grande narrazione è una storia su come, una volta, le persone vivevano in sistemi di relazioni sociali intense all'interno di comunità di solito consolidate da un qualche sistema di parentela. Negli ultimi due secoli una combinazione di forze fra cui il capitalismo, l'industrializzazione e l'urbanizzazione ha provocato un indebolirsi del tessuto di queste forze sociali. Questo indebolimento ha portato da un lato a uno sviluppo proporzionale di nuove forme di autonomia personale e di individualismo, ma alla solitudine, all'isolamento e alla frammentazione sociale dall'altro. Ciascun teorico sociale ha dato a tutto questo una declinazione differente. Per esempio, de Tocqueville ${ }^{4}$ ha scritto sull'ambivalenza dell'individualismo come forma di pensiero sociale e politico. Durkheim ha messo in opposizione l'olismo e l'individualismo, mentre Marx ha visto un potenziale rivoluzionario in questo ritrarsi delle forze conservatrici. I sociologi come Giddens, più di recente, discutono alcuni aspetti della particolare natura di questo individualismo moderno - per esempio, il modo in cui le persone si muovono verso un sé basato su una narrazione più auto-consapevole di se stessi ${ }^{5}$.

Questa presupposizione accademica è supportata in pieno dagli argomenti più forti del giornalismo popolare moderno. L'idea che i social media stanno contribuendo a portare avanti il declino dei valori comunitari e sociali e al corrispondente emergere di individui egocentrici e narcisisti, è forse 
l'unico argomento molto comune negli articoli di quotidiani chiaramente destinato a dare risposta ai timori popolari, ma anche a crearne ${ }^{6}$. Inoltre, che i social media siano un ulteriore passo avanti verso l'espressione di sé individualizzati o verso un ritrarsi dalla vita sociale sono state affermazioni molto comuni fra i nostri informatori in diversi dei nostri siti. Forse questo è stato particolarmente vero in Inghilterra, benché là fosse spesso mescolato al timore persino maggiore di perdere la privacy.

Nel Capitolo 2 abbiamo esaminato le principali scuole di sociologia che ci sono parse rilevanti rispetto alla nascita dei social media. Nessuna si adatta facilmente a questo percorso. Castells si è più occupato di argomenti relativi alla nascita di una nuova era di "capitalismo dell' informazione" . Il lavoro di Rainie e Wellman è invece più rilevante. Essi hanno sostenuto un susseguirsi di tre rivoluzioni guidate da spettacolari avanzamenti nella tecnologia e nella comunicazione, ossia la Social Network Revolution, l'Internet Revolution e la Mobile Revolution. Rainie e Wellman hanno fatto un lavoro importante, mettendo in discussione il racconto semplicistico presentato dai giornali e non considerando la nascita di network individualizzati come un ritrarsi dalla vita sociale. Si è trattato, piuttosto, di un potenziamento spesso legato ad un aumento della socialità offline.

Al contempo, essi sostengono che c'è stato un indebolimento dei legami tradizionali basati sui gruppi, sostituiti da un fenomeno che descrivono come "individualismo di rete". I due autori vedono il mondo sociale come un concatenarsi di reti" egocentrate in cui " $i$ new media sono il nuovo vicinato" ${ }^{10}$; qui "le persone sono consapevoli di essere individui, ma sono dentro le reti" ${ }^{11}$. Precisano, tuttavia, che questo non segna un passaggio verso l'isolamento sociale, piuttosto verso un'autonomia flessibile ${ }^{12}$. Sia Castell sia Wellman, in modi diversi, cercano di andare oltre questo dualismo individuale / sociale mediante un focus sulle reti.

Per sintetizzare i risultati descritti in questo capitolo, presenteremo alcuni esempi che sembrano in accordo con le argomentazioni di Rainie e Wellman. Le nostre scoperte e le nostre generalizzazioni più condivise sono però in forte contrasto con la loro posizione. Invece di vedere i social media come un proseguimento delle tendenze che si sono sviluppate attorno alla nascita di Internet, abbiamo molti esempi che sembrano andare in direzione contraria. La nostra definizione generale più importane di social media in termini di socialità modulabile suggerisce che i social media possano rappresentare un aumento, benché flessibile, dell'orientamento ai gruppi, e 
non, come è apparso agli esordi di internet, un continuo emergere di reti egocentrate. Questi gruppi possono essere tradizionali, come le caste e la famiglia indiane, o nuovi, come nel caso degli operai cinesi. In ogni caso, una caratteristica chiave dei social media è la loro capacità di porre rimedio alle separazioni che la vita moderna ha provocato nei gruppi tradizionali come la famiglia, e di agevolare un ritorno verso il gruppo. Il punto è: perché e quando le tendenze sembrano preferire la traiettoria che Rainie e Wellman descrivono, e perché e quando sembrano invece andare in direzione opposta. Insieme a loro, contestiamo l'idea che i social media debbano necessariamente renderci, a seconda dei casi, più o meno sociali, anche se qualche volta lo fanno ${ }^{13}$. Esaminiamo invece gli effetti più specifici dei social media su come socializziamo, e i modi in cui gli individui e i gruppi si relazionano reciprocamente attraverso questo medium.

Vale anche la pena di notare che il concetto di gruppo in sé non si oppone a quello di rete basata sull'individuo. In effetti, l'antitesi classica all'idea dell'individualismo frammentato della società contemporanea è stato il focus dell'antropologia della parentela come forma primaria di organizzazione sociale delle popolazioni tribali e di altre società di piccola scala ${ }^{14}$. Tutte le società però sono fatte di individui, e uno dei modi più comuni di rappresentare la parentela è stato in termini di una rete individuale di relazioni basata su Ego. Un tratto distintivo dell'antropologia, in opposizione alla psicologia o all'economia, è che non si considerano le persone come individui: si considera invece l'individuo come un ambito di networking sociale. La differenza stava nel grado in cui questo a sua volta definiva la posizione e l'identità di quell'individuo, e nel grado in cui questo era prescritto dalla cultura. Nei sistemi tradizionali di parentela gli obblighi sociali di una persona derivano dal fatto di essere figlio del fratello della madre, e non da un network di amici scelto individualmente che determina il grado di obbligo reciproco.

\section{Che differenza fanno i social media?}

Tornando ai nostri risultati, nel nostro sito italiano, in termini generali, fintanto che le vite offline delle persone erano per lo più soddisfacenti i social media sono stati usati poco. É solo quando esse hanno provato difficoltà o insoddisfazione nella loro vita che i social media sono potuti diventare un 
modo importante di affrontarle. Per esempio, le madri single, in genere, sono molto più attive su Facebook delle loro coetanee, e dai loro amici online ottengono un sostegno affidabile e crescente. Le famiglie separate spesso usano una combinazione di Facebook, Skype e WhatsApp per ricostruire gli spazi domestici. Quindi, in questo sito italiano, i social media hanno principalmente senso come complemento, o anche spesso come modo per rimettere in sesto la vita sociale offline.

"Ristabilire" è un tema comune, dato che molte persone in molti posti sentono che l'intensità delle relazioni sociali associata a un ideale comunitario del passato si è persa nella vita moderna. Questo si percepisce come l'esito di un individualismo crescente, che riflette le trasformazioni della politica economica moderna, insieme a un aumento della migrazione, più lunghe migrazioni interne per lavoro e molti altri casi di separazione sociale. Di conseguenza, in alcune società esiste il timore diffuso che la socialità stessa sia qualcosa che stiamo perdendo, o di fatto abbiamo perso, e che l'individualismo moderno concede meno e ha scarso interesse per istituzioni come la famiglia o la nazione, rappresentando uno slittamento verso il proprio interesse individuale. Non sorprende allora che i social media siano visti come potenzialmente in grado di riaffermare la connettività sociale.

Una delle ragioni principali per cui nel nostro sito inglese le persone usavano i social media come Facebook, e prima le piattaforme come Friends Reunited, era proprio per compensare un percepito indebolimento della socialità. I social media sono importanti anche per le neo mamme; li usano per creare nuovi social network con altre mamme in un periodo in cui hanno bisogno di sostengo e altrimenti rimarrebbero isolate in casa. I giovani del nostro sito brasiliano trovano anche conforto nell'idea che i social media consentiranno loro di mantenere i contatti con i loro amici mentre vanno incontro a nuove occasioni nel mondo dell'istruzione, del lavoro o altro. Un simile punto, porre rimedio alla lontananza, è centrale anche nell'uso dei social media da parte delle famiglie transnazionali di Trinidad.

La situazione è più complessa nel sito in Turchia sudorientale, dove i social media stanno esercitando un impatto molto più profondo sul modo in cui le persone socializzano. Per esempio, dove tradizionalmente le famiglie limitavano la libertà di movimento delle giovani donne e le loro reti sociali, i social media sono diventati per loro la maniera principale di avviare nuove relazioni sociali, e anche individuali, fuori dalla famiglia. Alcune studentesse di scuola superiore contano centinaia di amici su Facebook; in alcuni casi 
usano falsi account per non essere riconosciute dai loro parenti o amici di famiglia. Come sottolineato nel Capitolo 8 , nelle società musulmane l'onore e il valore degli uomini sono tradizionalmente dipesi dalla capacità di controllare i movimenti e le reti sociali delle donne; adesso, i social media stanno offrendo alle donne l'occasione di sfuggire a tali controlli. La capacità di impegnarsi in comunicazioni online ininterrotte fra giovani donne e uomini, il che implica anche l'innamoramento, prima era rigorosamente ristretta ai partner nel periodo pre-matrimoniale.

Questa sarebbe una prova a sostengo dell'aumento di network individualizzati secondo quanto descritto da Rainie e Wellman, ma anche questo caso non va a discapito dei legami di gruppo. Specie nel caso della popolazione curda, l'effetto primario è riprodurre forme tradizionali di organizzazione della parentela. I curdi usano ampiamente Facebook e WhatsApp per tenersi in contatto con parenti distanti che vivono in altre città in Turchia e all'estero. I giovani curdi spesso hanno centinaia di parenti con cui sono in contatto su Facebook. Sono di solito coetanei dello stesso sesso che, insieme ai fratelli dell'altro sesso e ai parenti più grandi che non sono connessi a Internet, costituiscono un intero lignaggio. Diversi di questi lignaggi formano così una tribù, storicamente l'unità sociale primaria della società curda. Alcuni di questi parenti non si sono mai incontrati di persona. I social media sono diventati uno spazio online dove uomini e donne si possono sentire parte della famiglia estesa, del lignaggio o della tribù anche solo passando un po' di tempo a guardare le immagini e le fotografie, e a leggere le notizie sui loro parenti. I social media hanno in questo modo configurato forme nuove, moderne, di alleanza tribale. In parte questo compensa migrazioni e migrazioni forzate per cui le persone non vivono più vicine le une alle altre, e così c'è ancora un modo per rimediare.

Analogamente, nel nostro sito indiano la società è organizzata sulla base della stretta osservanza di una serie di categorizzazioni sociali che includono la parentela, l'età, il genere, la classe e la casta. Molte di queste categorie tradizionali si trovano a loro volta sui social media, tanto che - almeno per quanto riguarda le classi e le caste inferiori - i social media si possono a ragione definire "i media della parentela". L'uso altamente socializzato dei social media si estende fino all'utilizzo delle piattaforme anche per controllare altri membri all'interno della medesima famiglia o casta. In questo senso è difficile per le persone usare i social media per affrancarsi online come individui autonomi, a meno di non usare profili falsi, cosa che alcuni fanno, 
anche se si tratta di eccezioni e non della norma. I social media agiscono per mettere a freno precedenti spinte verso l'individualismo e lontano dal gruppo. A volte questo senso di tradizione e abitudine sociale è decisamente esplicito. In particolare, è così nel nostro sito nella Cina rurale, dato che si trova vicino al luogo di nascita di Confucio e i locali avvertono ancora una forte affinità - dettata da questo e altri fattori - con una filosofia centrata sull'ideale del conformismo sociale e sul valore del rispetto verso genitori e anziani.

Comune a tutti e tre i casi c'è che i modi in cui i social media alimentano la socialità sono relativi alle condizioni della precedente socialità offline. In Italia, spesso le persone semplicemente non hanno bisogno di usare strumenti online per creare questa socialità. Nella Turchia sudorientale, d'altro lato, soprattutto i giovani si sono misurati con le nuove possibilità offerte dai social, sia per porre rimedio a relazioni interrotte dalle separazioni sia per creare nuove forme di networking individualizzato, che di conseguenza hanno alterato profondamente le loro vite.

Il problema è che non si può ignorare il retroterra originario di questa discussione che ci pone nella posizione di contrastare le argomentazioni a favore di una tendenza generale all'individualismo, solo perché questo tipo di narrazione domina il giornalismo e la percezione popolare dell'impatto dei social media. Un genere nuovo sui social media come il selfie viene quindi automaticamente visto come un ulteriore esempio di individualismo emergente. Anche negli studi accademici è presente l'idea che il selfie debba implicare una crescente espressione di sé. Tuttavia, come abbiamo visto nel Capitolo 11, studiando il selfie etnograficamente, ancora una volta troviamo una maggiore continuità con i tradizionali legami fra i gruppi piuttosto che mere autorappresentazioni. In prospettiva più generale, non troviamo prove per proporre che i selfie abbiano un'associazione speciale con le società più individualiste. I selfie sono spesso un mezzo per mostrare il proprio legame con la famiglia o con la chiesa (come nelle Filippine) ${ }^{16}$. Chiaramente il selfie è anche parte dell'auto-espressione dei teenager e del loro profondo interesse per come appaiono, ma questo non significa necessariamente che il selfie abbia creato o anche accentuato questo tipo di attenzione.

Nel nord del Cile l'aumento di visibilità consentito dai social media pubblici funzionava come un mezzo per cui i membri della comunità si attribuivano reciproco riconoscimento, fiducia e appartenenza alla comunità. Nel rendere visibili le relazioni, le persone sottolineavano la famiglia e l'amicizia, 
consolidando e allargando realmente questi legami. I selfie quotidiani erano considerati parte integrante di questa socialità, insieme all'autoderisione, alle battute fra amici e all'intenso scambio di commenti. Tali dati non fanno che rafforzare la semantica dei social media in quanto quasi sempre "social".

\section{Conformismo e collettività: "perché lo fanno tutti"}

Il nostro progetto collettivo ha raccolto notevole evidenza del fatto che i social media tendono a dare maggiore visibilità alle cose - e quindi diventano un mezzo potente per creare e mantenere il conformismo. I genitori, nel sito italiano, incoraggiano i figli a entrare nei social media per essere più simili ai loro coetanei. In entrambi i siti cinesi le persone condividono pensieri idealistici su WeChat e QQ perché lo fanno tutti gli altri. Quasi ovunque le persone mostrano un supporto reciproco costante mediante i "mi piace" e i commenti, nel desiderio di associarsi a persone simili a loro, o di renderle più simili a loro.

Questo è stato particolarmente chiaro nel sito cileno. Le persone là spesso prendono parte a un dialogo semi-pubblico sui loro profili social, visibile agli amici degli amici che si uniscono al genere più diffuso dello scambio di scherzi. Le prese in giro e gli insulti per gioco legano un pubblico più ampio all'interno di un sapere culturale condiviso. Questi scambi umoristici quasi sempre avvengono sulla base di un ampio uso di convenzioni linguistiche tipo slang, che consente alle persone di marcare la loro autenticità in quanto membri della medesima comunità linguistica cilena. Gli individui che non si conoscono diventano così conoscenti e, a volte, in seguito, amici attraverso questi scambi. A volte, diventare amici su Facebook porta le persone ad avviare attività di svago faccia a faccia, per esempio mangiare insieme, praticare sport o uscire. Avere conoscenze più locali aiuta anche a trovare un nuovo lavoro, comprare e vendere online oggetti usati e a cercare potenziali partner.

Le questioni presentate nei capitoli precedenti sull'impatto conservativo dei social media pubblici rispetto al genere e alla politica sono altrettanti esempi di questo uso dei social media a garanzia del conformismo. Un tale senso di conformismo è evidente nel sito dell'Italia del sud, dove le persone hanno in gran parte rifiutato le piattaforme che considerano individualistiche come Twitter e Instagram. Le persone vogliono esprimere non differen- 
ziazione individuale ma collettiva, per esempio un senso sociale di bellezza ${ }^{17}$, che si può trovare nel paesaggio locale ma anche nell'adozione collettiva di stili e accessori promossi da professionisti locali e nazionali, come designer, stilisti e acconciatori.

Roberta, per esempio, è una donna di circa 40 anni sposata e madre di due figli. Come molte donne della sua età, ci tiene a essere ben vestita con uno stile personale e ad avere una casa perfettamente in ordine e curata. Va almeno una volta al mese da un parrucchiere che conosce da molti anni e che le consiglierà l'acconciatura di moda per la stagione. Così, Roberta acquista sempre occhiali da sole e da lettura raccomandati dal suo ottico, che sa ciò che va di moda. Stare al passo con la moda si fa non per emergere individualmente, ma per conformarsi agli stili delle altre donne della cittadina che hanno la stessa posizione sociale. Essendo sposate, queste donne possono astenersi dal postare loro fotografie su Facebook. Usano invece WhatsApp per condividere immagini di vestiti, tagli di capelli e accessori, con i familiari e le loro pari.

Mentre questi modelli di consumo nel sito italiano sono stabili, si trasformano in forme più dinamiche di aspirazione nel sito del Brasile, caratterizzato dalla presenza di una classe sociale emergente. In Brasile le grigliate, le auto, i vestiti firmati e i musicisti costosi sono sempre stati associati con la ricchezza e il potere, e i post che li ritraggono sono spesso modi per affermare mobilità verso l'alto, un argomento discusso nel Capitolo 12. Tuttavia, i dati da Trinidad mostrano che questa affermazione va moderata. Anche se apparire bene a Trinidad è importante, i post su Facebook che indicano che un individuo cerca di apparire migliore di chiunque altro o di emergere troppo attirano insulti e ironia, il che si configura ancora una volta come un freno a ogni tendenza all'individualismo. Ciò suggerisce che i social media si possono utilizzare per la differenziazione e la mobilità, ma questo può non avere a che fare con l'individualismo dal moneto che tutto è messo in mostra sempre sotto la censura morale della conformità sociale. Punto comune è che i social media rendono la creazione e il mantenimento del conformismo molto facili.

\section{Privacy e socievolezza}

Di tutte le discussioni sull'impatto dei social media, le riflessioni su privacy e social media rappresentano il modo migliore per sottolineare come persone di posti differenti la pensino molto diversamente su ciò che, per certi 
aspetti, si ritiene un concetto comune. Il dibattito molto acceso sulla privacy copre numerose e diverse dimensioni ${ }^{18}$. Una riguarda i dati personali ricavati dalle aziende di social media e usati a scopo commerciale, oppure per il controllo da parte dello stato. In pratica, questo timore si è rivelato molto meno evidente nei nostri siti di quanto si ritenesse. Le persone nel nostro sito in Turchia sudorientale parlavano sì di una potenziale perdita di privacy, ma erano anche preoccupate di cosa potesse accadere alle loro informazioni personali in una situazione di forte conflitto politico. Nel Capitolo 5 abbiamo mostrato come le persone nel sito inglese siano infastidite dall'uso commerciale dei loro dati per la pubblicità mirata. Considerando però il nostro progetto complessivamente, questo tipo di timori sono stati poco espressi.

Al centro di questa discussione ci stanno i presupposti dei dibattiti iniziali in Europa e in America del Nord: la convinzione che la privacy è una sorta di condizione naturale ora messa a rischio dalla visibilità online ${ }^{19}$. Nel sito inglese era constante e ripetuto il timore riguardo al possibile uso dei social media per scavare nella vita privata di ciascuno. In Cina la situazione è molto diversa. Già in prospettiva storica, il modo locale di intendere tali argomenti appariva diverso dal concetto occidentale di "privacy" nell'ambito della vita sociale tradizionale ${ }^{20}$. Anche oggi, mentre nelle aree urbane della Cina la gente ha forse maggiore familiarità con il concetto di "yinsi" (privacy), nelle aree rurali la "privacy" si considera un termine di moda o occidentale. La famiglia rurale tradizionale era un'unità più collettiva, in cui non esisteva che le persone potessero volere o aver bisogno di uno spazio privato. Non avevano questo "frame" di attesa di privacy.

Anche nel sito della Cina industriale gli operai vivono o insieme dentro dormitori collettivi per i lavoratori, in sei o otto in una stanza, oppure con un'intera famiglia in stanze in affitto. Cucinano in spazi collettivi e entrano uno nella stanza dell'altro senza bussare. La vita quotidiana è anch'essa decisamente in comune. È convinzione diffusa che una persona cercherebbe di nascondere allo sguardo pubblico solo un brutto segreto. In un contesto siffatto, l'uso dei social media, soprattutto per il fatto che esso, in Cina, è in gran parte anonimo, è diventato una delle prime occasioni di riconoscimento e di esperienza di una propria privacy. Sui social gli operai migranti possono annotare i loro pensieri e i loro segreti entro un ambito relativamente privato $^{21}$, senza preoccuparsi dei giudizi istantanei delle persone che vivono nello spazio offline in comune. Evidentemente, in questo caso, la tecnologia digitale ha avuto un impatto significativo sulle vite degli operai cinesi, e in 
un modo che va esattamente all'opposto rispetto a quei dibattiti che danno per scontato che i social media rappresentano la distruzione di ogni privacy.

Un aspetto piuttosto diverso di queste discussioni sulla privacy proviene da una tesi comune sin dagli esordi di internet: andare online era per certi aspetti uno sviluppo antisociale ${ }^{22}$. Il punto è stato concretamente criticato da Hampton e Wellman, che hanno scoperto che la socialità online poteva altrettanto efficacemente far crescere quella offline ${ }^{23}$. Ciò apre a una questione più generale: in che modo questa socialità online si riflette sulla socialità offline? Per fare un esempio, nel sito in Italia del sud le persone scarsamente istruite in genere non usano i social media per essere più social, mentre quelle con livelli più alti di istruzione, e che hanno vissuto in grandi città come Milano, Roma, Bologna, in effetti lo fanno. Il motivo è che gli appartenenti al primo gruppo non hanno ambizioni cosmopolite. Per loro ha più senso sottolineare le loro relazioni sociali offline, molto di più che quelle online. Tuttavia, i loro vicini più cosmopoliti vogliono usare i social media per tenere i loro contatti più estesi con persone di altri spazi urbani - se non altro, questi utenti desiderano attenuare il fatto di essere associati con ciò che percepiscono come l'opprimente connettività della società locale. Ciò che hanno in comune questi due gruppi è che entrambi vogliono usare i social media e le nuove tecnologie digitali per rendere le loro relazioni sociali più significative, in entrambi i contesti online e offline.

C'è sempre stata una contraddizione implicita nell'affermazione comune che la nostra crescente devozione per gli schermi ci avrebbe reso antisociali. Aveva più senso quando, per esempio, giocare al computer era praticato in grandissima parte da individui isolati. Tuttavia, quando oggi le persone usano prevalentemente dispositivi proprio per socializzare con altre persone mediante i social media (oppure tramite giochi di gruppo), quella critica lascia molto perplessi. Il fatto che le persone nei nostri siti in Turchia, Trinidad e in Brasile trascorrono grandi quantità di tempo cercando i profili social di altre persone non significa che davvero parlino di meno nelle interazioni faccia a faccia. Al contrario: sullo schermo trovano più argomenti che hanno in comune e che possono trattare nelle conversazioni dirette. Inoltre, con le webcam, la distinzione fra conversazioni online e offline è essa stessa diventata un po' confusa, specie quando le persone usano quelle che si definiscono webcam "always-on" e che restano sullo sfondo anche quando nessuno ci parla direttamente. Questo è uno degli esempi più chiari in cui ha senso parlare di persone che convivono, ma solo in rete ${ }^{24}$. 
Il fatto che nel nostro sito italiano la maggior parte delle persone non controlli tanto spesso direttamente i reciproci profili di Facebook non significa che esse non siano interessate ai loro pari o non cerchino di allargare le loro conoscenze. In Italia del sud, l'alto tasso di disoccupazione e i lunghi tempi di riposo non hanno prodotto maggiore uso dei social media. In altri posti, il tempo libero sarebbe ritenuto adatto per il networking online. Però non è così che si intende il riposo. Le persone, invece, collegano la socializzazione online con le loro tradizioni di socialità offline legate a particolari momenti e situazioni, per esempio quando si è al lavoro, quando si prende un caffè, o in piazza la sera. Le persone che trascorrono più tempo di altri a guardare i profili Facebook sono allora, per lo più, commessi o impiegati pubblici che lo fanno durante i loro momenti di inattività sul lavoro, proprio come quando chattano.

Abbiamo sostenuto molte volte che l'uso dei social media riflette il modo in cui le persone intendono la socialità e le relazioni sociali nei loro rispettivi contesti locali, per esempio attraverso il prisma dei diversi concetti di privacy oppure, in alcuni casi, come un modo per promuovere il conformismo sociale. Vogliamo ora analizzare una delle nostre principali scoperte, che riguarda il modo in cui le persone usano i social media per regolare le loro relazioni effettive, per esempio rendendole più o meno visibili, con l'intenzione primaria di creare un senso di equilibrio nella loro socialità.

\section{Trovare un equilibrio: usare i social media per regolare le relazioni}

Invece di vedere i social media come un modo per diventare o più social o più individualisti, le persone del nostro villaggio inglese usano le piattaforme di social media, come Facebook, in primis per tenere le persone alla giusta distanza ${ }^{25}$. Possono calibrare le loro relazioni in molti modi differenti, in quanto forma di socialità modulabile. Si può andare dal commento costante fino al "mi piace" occasionale ai post di un'altra persona; dall'inserirla dentro un piccolo gruppo di WhatsApp fino al semplice riconoscimento come follower su Twitter. Da lì ci si può muovere in direzione di relazioni più personali tramite messaggi privati e chiamate telefoniche o in direzione di relazioni essenzialmente professionali tramite LinkedIn e email. Ciò esemplifica il concetto più generale di socialità modulabile.

Si possono sfruttare a tale scopo caratteristiche dei social media come 
l'a-sincronicità, come quando le persone decidono di rispondere subito agli amici stretti e con più calma alle persone che non desiderano incoraggiare in modo particolare nella comunicazione troppo frequente. Un certo ritardo di replica ai messaggi sui social media è accettabile, le persone hanno tempo sufficiente per gestire strategicamente i loro modi di presentarsi ${ }^{26}$. In luoghi come la Cina industriale, i migranti tendono a limitare gli argomenti delle telefonate quotidiane fatte a familiari e amici nelle città d'origine, spesso molto lontane, e parlano di denaro, soluzioni a problemi finanziari e pettegolezzi. Considerazioni sui desideri personali e la vita in generale si considerano "non affrontabili" tramite conversazioni telefoniche, e per questo sono messe su QQ dove appaiono più adatte.

Le persone spesso usano i social media anche per negoziare un equilibrio tra persone che conoscono e il potenziale ruolo di estranei ${ }^{27}$. Data l'importanza notevole del rispetto per la tradizione nel nostro sito nella Cina rurale, le persone erano spesso in imbarazzo nel dichiarare che usavano le loro pagine sui social media per comunicare solo con persone che conoscevano dal paese d'origine, o di altre reti come la famiglia, il lavoro o la scuola. Tuttavia, McDonald ha trovato che questo era in contraddizione con il loro uso di funzioni all'interno delle più popolari piattaforme di social, che consentivano loro di connettersi con perfetti sconosciuti. Grazie a questo mezzo si impegnavano in un tipo di socialità del tutta nuovo che in precedenza sarebbe stata impensabile. Spesso è proprio questo che rende le persone curiose di tali incontri. Dal momento che possono anche ottenere un grado di anonimità prima impossibile, ciò diventa un altro esempio del modo in cui i Cinesi usano i social media per provare ciò che per loro è un'idea di privacy ampiamente inedita. Oltre alla socialità modulabile, le persone hanno anche un senso di scala che va dalle maniere di socializzare più tradizionali fino alle più moderne. Di nuovo, si può cercare qui un equilibrio, usando i social media sia per le forme di socializzazione completamente innovative sia anche per quelle fortemente tradizionali. Un altro esempio è l'uso prima descritto dei social media in Turchia sudorientale, sia per nuovi tipi di flirt e di chat individuali sia per un rinsaldamento dei legami con i gruppi tradizionali come la tribù.

Questo concetto di equilibrio inoltre ci riporta a un nostro punto precedente, evidente negli studi sulle subculture giovanili: in generale, diventare individui è un progetto sociale ${ }^{28}$. Come i punk o $\mathrm{i}$ ghot, i giovani considerano se stessi come soggetti assertivi dell'individualità contro l'assetto sociale 
dominante, ma dall'esterno essi sono visti come conformi all'estetica della subcultura che hanno scelto. Possono anche essere interessati alla moda e al cambiamento, ma l'adattamento a questi processi avviene sempre sulla base dell'appartenenza a un gruppo. I social media li aiutano in una misura prima semplicemente impossibile, perché l'aumento della visibilità significa che oggi è più chiaro cosa fanno gli altri e come appaiono. È persino più facile diventare un punk o un ghot individualizzato che sembra proprio come gli altri punk e ghot. Molte delle considerazioni di questo capitolo qui convergono. Se consideriamo l'espressione di molte versioni di individualismo come la dimostrazione di fatto del modo in cui i social media si possono usare per rafforzare il conformismo sociale, allora siamo arrivati molto lontano rispetto alla semplice opposizione, da cui siamo partiti, fra individuale e sociale.

$\mathrm{Ci}$ sono quindi tre principali conclusioni da trarre. Abbiamo visto come la visibilità dei social media sostenga il progetto della conformità sociale, anche quando le persone si ritengono utenti dei social per esprimere individualità. Abbiamo anche visto come i social media in quanto socialità modulabile offrano una serie di meccanismi attraverso cui le persone possono trovare un equilibrio, per esempio, tra tenere gli altri o vicini o a distanza, o tra forme di socialità nuove oppure tradizionali. Tuttavia, c'è una terza generalizzazione che emerge dall'analisi comparativa. Laddove le persone sentono declinare la socialità comunitaria, allora la componente di gruppo dei social media può essere vista come un modo per mantenere o rafforzare la socialità di gruppo della famiglia, della casta o della tribù. In molto casi si tratta di un tentativo decisamente deliberato di arrestare ciò che si percepisce come un indebolimento degli orientamenti tradizionali verso la società e verso i gruppi come la famiglia. All'opposto, laddove le persone trovano opprimenti il conformismo sociale o la comunità come, per esempio, nel caso dei vincoli relativi al comportamento delle donne in numerose società, allora i social media consentono un certo movimento verso il networking individualizzato. 



\section{Capitolo 13 \\ I social media rendono la gente più felice?}

Per gli antropologi, valutare se qualcosa rende la gente felice è un compito complesso se non impossibile. I modi ampiamente variabili in cui gli individui concepiscono la felicità spesso si allineano a più ampi ideali culturali e sistemi di valori associati con la classe, la religione, il genere, l'età, certe prospettive filosofiche, il livello d'istruzione e qualsiasi altra caratteristica collettiva. Oltre a questi orientamenti culturali alla "felicità", gli individui hanno sensibilità variabili rispetto a ciò che li rende felici, che quasi certamente cambiano anch'esse nel corso del tempo. Inoltre, non è del tutto chiaro da che distanza dovremmo osservare le affermazioni di felicità delle come prova di quanto effettivamente sono felici. Per circoscrivere una domanda sulla felicità, quindi, dobbiamo considerare non solo quali fattori culturali formano la felicità, ma anche come gli individui interpretano le domande sulla felicità e quali discorsi sulla felicità sono accettabili pubblicamente. Per esempio, dobbiamo considerare se per ogni contesto culturale la felicità si riferisca a gratificazioni momentanee o a soddisfacimenti che durano tutta la vita, a un leggero miglioramento dell'umore o a un più profondo senso di realizzazione del sé. Le persone considerano la felicità come qualcosa che arriva da un concetto metafisico come la religione o la natura, le relazioni umane o persino i beni materiali? Sarebbe volgare pretendere di essere felice, o sarebbe volgare o ingrato pretendere di essere qualsiasi cosa eccetto che felice? Tutto ciò influenzerà profondamente le risposte individuali a qualsiasi domanda sulla felicità.

Il relativismo culturale implicito nel termine felicità può spiegare perché gli antropologi sono stati storicamente riluttanti a intraprendere quella che si potrebbe definire una "ricerca sulla felicità". Questo è uno di quei capitoli che abbiamo incluso perché abbiamo sentito il dovere di rispondere alle domande che le persone in genere ci pongono, ma anche a quelle a cui vorremmo rispondere. Eppure, anche dalla nostra prospettiva approcciare questo tipo di argomento mantiene un certo significato, in particolare nel contesto di un cambiamento tecnologico.

Se la modernità avrebbe portato più o meno felicità è tema di dibattito almeno a partire da Rousseau ${ }^{2}$, che sosteneva che la civilizzazione si era ac- 
compagnata a una perdita di autentica felicità. La nozione di felicità è anche significativa nel lavoro di Durkheim ${ }^{3}$, James ${ }^{4}$, Weber ${ }^{5}$, Locke $^{6}$ e Comte ${ }^{7}$, che hanno tutti riflettuto sui vari modi in cui la modernità influenza le nozioni di felicità e individualità. Come però argomenta Thin ${ }^{8}$, l'antropologia del ventesimo secolo (e ora del ventunesimo) è stata ampiamente silente sul tema, in parte perché il relativismo culturale ha scoraggiato le comparazioni interculturali ${ }^{9}$.

Valutazioni interculturali della felicità sono state invece l'ambito di economisti come $\operatorname{Sen}^{10}$, che suggerisce che le misurazioni del benessere basate sul PIL ${ }^{11}$ dovrebbero essere integrate con indicazioni quali l'Indice di Felicità Interna Lorda (Gross National Happiness) ${ }^{12}$ o il World Happiness Report $^{13}$. Questi prendono in considerazione fattori come la salute, il livello d'istruzione e altri indicatori dello "sviluppo umano" definiti dalle Nazioni Unite $^{14}$. Tali indici seguono Maslow ${ }^{15}$ nel riconoscere che la felicità dipende da molto di più che da necessità fisiologiche e di sicurezza; comprende emozioni positive e negative, così come sentimenti di scopo o di significato di vita ${ }^{16}$. Includere la "felicità" nella valutazione del benessere è certamente preferibile a una semplice misura economica, ma queste ampie misure quantitative ci dicono poco sulle differenze locali e individuali nel definire la felicità.

In quanto antropologi, studiare la felicità comparativamente facendo etnografia conferisce a tale questione maggiore profondità, ma pone anche il problema di comparare un concetto diversamente definito a seconda dei contesti. Come punto di partenza prendiamo l'ampia definizione di felicità di Thin ${ }^{17}$ che delinea tre significati generali: motivazione, valutazione e emozione. Nella sua formulazione, la motivazione è correlata all'ambizione e a come si percepisce lo scopo della vita. La valutazione è riferita alle percezioni che gli individui hanno della loro personale qualità di vita in relazione ai valori culturali e alla moralità. L'emozione è associata ai piaceri temporanei e a stati emotivi più duraturi di benessere. La felicità allora comprende sensazioni, espressioni e resoconti emotivi, così come le storie importanti che le persone raccontano, le relazioni che costruiscono e le loro preferenze estetiche ${ }^{18}$.

La definizione di Thin comporta non solo la variazione culturale nei concetti di felicità, ma anche l'emozione individuale e la variazione entro questi concetti. La condotta morale in un luogo può essere strettamente legata alla religione, in un altro possono essere dominanti fini più umanistici di istru- 
zione o di consapevolezza ambientale. I modi in cui le persone cercano il piacere può implicare estetiche, sessualità, relazioni sociali, cibo, manifestazioni e feste, droga, trasformazioni religiose, consumo, avventura o diverse loro combinazioni.

\section{Studi sulla felicità e i social media}

Anche se l'antropologia può evitare questo tipo di argomento, i dibattiti pubblici sulla relazione fra social media e felicità personale (o più spesso, infelicità) sono ampi e sempre più presenti in pubblicazioni di alto profilo. Negli ultimi cinque anni, numerose agenzie d'informazione (compresi Time Forbes, The Huffington Post, The New Yorker e The Daily Mirror) hanno citato studi psicologici che indicano che i social media possono far aumentare la depressione $^{19}$, l'insoddisfazione ${ }^{20}$, la gelosia ${ }^{21}$, l'immagine negativa del proprio corpo $^{22}$ e il senso di solitudine ${ }^{23}$. Questi studi attribuiscono le sensazioni negative a una varietà di motivi. A volte gli utenti possono pensare di essere ignorati dai loro contatti sui social media $^{24}$. In diversi studi gli utenti comparano la loro vita personale alle immagini della vita dei loro conoscenti, che vedono online $e^{25}$. In altri casi ancora, le persone si sentono depresse perché considerano il loro tempo su Facebook improduttivo ${ }^{26}$. Altri studi, però, criticano e contraddicono questi risultati ${ }^{27}$. Alcuni ritengono che usare i social network per chattare o fare programmi aumenta il senso di appagamento $^{28}$; si potrebbe scoprire una correlazione positiva fra uso di Facebook e aumento di capitale sociale, fiducia e impegno civile, e dunque un senso di gratificazione nella vita ${ }^{29}$. Anche Facebook ha condotto il suo studio controverso $^{30}$, da cui è risultato che quando agli utenti venivano mostrati post con più parole positive, i loro post ne contenevano di più e quando invece con meno parole positive, anche i loro post ne contenevano meno. I ricercatori hanno confermato che il "contagio emotivo" dei post di Facebook funzionava proprio come il contagio emotivo offline, dove la presenza di persone felici nella propria vita rende un individuo più felice, mentre persone negative lo rendono meno contento.

Il fatto che alcuni studi evidenzino conseguenze emotive negative dei social media mentre altri ne vedano il potenziale per un supporto emotivo positivo potrebbe semplicemente riflettere il fatto che le persone sui social fanno cose molto diverse. Un utente potrebbe infatti avere esperienze di- 
verse dei social media giorno per giorno o anche minuto per minuto. Le piattaforme di social media come Facebook o QQ non sono una cosa unica, ma prendono forme differenti a seconda dell' uso individuale. Possono essere usate per tenersi in contatto con la famiglia, mostrare le fotografie delle vacanze o sviluppare nuove amicizie sulla base dello scherzo o dell'emozione. Ovviamente, quando i social media giocano ruoli simbolicamente diversi in momenti diversi, non influenzano le emozioni in un modo unico e diretto ${ }^{31}$. Il nostro problema è che possiamo continuare ad asserire che chiedere se i social media rendano le persone più felici è una domanda troppo semplicistica, non possiamo però negare che la popolarità della domanda rivela un enorme interesse nell'impatto dei social media sulle emozioni delle persone. Questo vale non solo per il giornalismo. Domande del genere, se i social media ci rendano felici o infelici, o più in generale se siano buoni o cattivi per noi, oggi fanno del tutto parte delle conversazioni standard tra le persone in tutto il mondo.

Prendendo in considerazione questo interesse pubblico, ciascuno dei nostri studi dei nove ricercatori ha incluso una domanda sulla felicità nell'indagine sugli utenti dei social media ${ }^{32}$. Lo scopo non era sostenere o smentire i risultati di questi altri studi, piuttosto trasmettere il senso di come le risposte differissero ampiamente lungo le molteplici collocazioni geografiche. Infatti, come mostra la fig. 4.26, le risposte alla domanda "I social media ti rendono più felice?" 33 variano in modo significativo a seconda della collocazione.

Naturalmente, questi risultati non dovrebbero essere sovra-interpretati. È possibile che in Cina le persone sentano che l'associazione positiva con i new media è ciò che si suppone che loro stesse pensino, come riflesso della promozione che il governo cinese fa dei new media come avanguardia della modernità; ne risulta che rispondono in modo preponderante che i social media li rendono più felici. Analogamente, alcuni individui nel nostro sito in India del sud e in quello in Turchia sudorientale potrebbero sentire che attribuire felicità ai social media sminuisca la loro posizione rispetto ai sistemi di valori tradizionali associati con la famiglia, la casta o la tribù, come risulta da una percentuale relativamente alta di persone che affermano che i social media le rendono meno felici.

In molti dei siti, la maggioranza delle persone afferma che i social media non hanno fatto differenza nella loro felicità. Relativamente pochi seguono i resoconti più comuni sui media suggerendo vari modi in cui sono stati resi 
infelici. Calando nel contesto questi dati e esaminando i concetti di felicità nell'ambiente culturale degli individui, possiamo almeno apprendere qualcosa su ciò che è in gioco quando le persone fanno affermazioni riguardo alla felicità, anche se alla fine non possiamo determinare se i social media rendono le persone felici o meno.

\section{Capacità e aspirazioni}

Accesso a internet e social media spesso offrono alle persone maggiori possibilità, che siano per acquisire abilità professionali o per connettersi con persone nuove e idee o semplicemente per farsi sentire in pubblico. La connessione fra nuove possibilità, maggiori aspirazioni e aumento della felicità basato su ciò che Thin definisce "motivazione" tuttavia non è sempre nettamente chiara.

La nuova tecnologia spesso permette agli individui di immaginare vite diverse, o la "capacità di avere aspirazioni", che Appadurai ${ }^{34}$ sostiene sia un elemento chiave per l'empowerment dei poveri. Si potrebbe allora presumere che se i social media forniscono un accesso a nuove aspirazioni, e a volte danno maggior accesso a ciò cui le persone aspirano, è per questo che accrescono la felicità. Questa correlazione è cruciale nello studio di Wang ${ }^{35}$ degli operai cinesi, molti dei quali rappresentano il tipo di poveri ai quali Appadurai si riferisce. Questi migranti rurali trasformati in operai spesso usano QQ per rappresentare le loro fantasticherie di stili di vita "cool e moderni”. Come illustrato nella figura 11.1, le operarie postano foto di abiti nuziali e sogni di "principessa", mentre gli uomini preferiscono foto di rock star, donne sexy e costose auto sportive. In ogni caso le immagini servono da via di fuga dalle fatiche del lavoro in fabbrica.

Questo tipo di aspirazione però non è inevitabile. Nel campo di ricerca di Haynes $^{36}$ nel nord del Cile, popolato anch'esso da migranti per motivi economici che spesso pensano che la loro città sia un posto marginale, brutto e noioso, Facebook e Instagram non sono spazi per fantasticare, ma servono piuttosto per rappresentare le loro vite proprio come sono. Nelle Fig. 11.4 e 11.10 vediamo le fotografie e i memi che presentano le loro esistenze contenute entro ambizioni semplici, strettamente aderenti ai tipi di normalità mostrati dai loro vicini. Scacciano la noia postando su Instagram foto della coda per pagare la bolletta del telefono o del loro computer in funzione, con 
hashtag come \#instanbored. Anche i memi divertenti di cui vanno così spesso matti rappresentano le loro aspirazioni come minime, con foto di pietre tombali con l'iscrizione "La mia voglia di studiare" o i memi di Kermit la Rana della fig. 11.10.

A prima vista, i contesti di questi due siti appaiono simili, popolati di migranti in cerca di nuove opportunità di lavoro. Eppure, mentre nella Cina industriale gli individui migrano verso le aree relativamente sviluppate e più cosmopolite come Shanghai, il Cile del nord è spesso concepito dagli abitanti come "solo parzialmente incorporato nella nazione", come a dire che si sono spostati, sia fisicamente sia simbolicamente, dal centro cosmopolita verso la periferia ${ }^{37}$. Invece di ambire a una maggiore ricchezza, a un'istruzione più elevata o a social network più estesi, le ambizioni dei nord cileni riguardano più il benessere e il sostegno delle loro famiglie. I cinesi sono migrati verso il centro e i Cileni verso i margini, il che può in parte spiegare qualcosa delle differenze nelle loro aspirazioni. Per tornare ad Appadurai, questi due esempi indicano che il ruolo dei social media nella felicità, più che avere a che fare con la capacità di nuove aspirazioni, può mettere in luce come le aspirazioni riflettono il contesto più ampio sociale e economico in cui le persone vivono.

Un esempio del sito brasiliano di Spyer ${ }^{38}$ rende tutto ciò ancora più evidente. Spyer usa l'espressione "classe emergente" per descrivere gli abitanti del suo sito che si sono spostati anche loro per opportunità di lavoro. Abbiamo visto come i social media sono importanti per rappresentare la mobilità sociale. Le immagini del consumo prese in luoghi esclusivi come la palestra o la piscina, o che ritraggono vestiti di marche prestigiose, riflettono in forma materiale l'immaginazione degli individui sul loro futuro e su chi sognano di diventare. All'opposto, condividere una foto vicina a qualcosa di associato all'arretratezza, per esempio un muro di mattoni non terminato, è tabù. Aspirazioni elevate a volte portano a un aumento della pressione a soddisfarle, e alcuni individui temono di essere considerati come coloro che sono rimasti indietro rispetto al vicinato come chiunque altro si sposti. La mobilità sociale porta a una notevole competizione fra vicini, colleghi o compagni di scuola, e persino fra amici. Mentre i residenti considerano vantaggiose queste nuove capacità, essi pertanto si ricordano del passato come di un periodo di maggiore gratificazione, un tempo in cui tutti si conoscevano, i giovani erano più rispettosi, criminalità e violenza erano minimi e il denaro non era una preoccupazione quotidiana. Benché le nuove capacità 
e le nuove aspirazioni connesse ai social media possano in certi casi portare a immaginare una felicità più grande, possono anche catalizzare le nuove pressioni e i fallimenti che complicano questa narrazione.

\section{Valori sociali duraturi}

In ognuno di questi casi le persone associano il cambiamento alla distruzione di valori, all'alienazione e alla rarefazione di ideali sociali. Possono allora guardare ai social media per rafforzare ciò che percepiscono come i loro valori tradizionali e stili di vita, vitali per preservare ciò che considerano come condizioni per la felicità. In tali casi, i social media possono essere usati non per incrementare le capacità, ma per reiterare gli ideali convenzionali. Per esempio, nel sito di McDonald ${ }^{39}$ nella Cina rurale, la rettitudine e l'adesione ai valori morali sono molto considerate e vengono viste come derivanti in parte dalla storia di ambito confuciano, taoista e buddista, oltre che in relazione con lo stato cinese moderno.

Gli individui spesso usano i social media per propagare una vita morale, rispondente alle preoccupazioni sia proprie sia del governo cinese relative al fatto che le nuove tecnologie "straniere" come internet potrebbero essere incompatibili con i valori morali e con i modi di agire corretti, e potrebbero avere effetti negativi sui valori sociali cinesi ${ }^{40}$. Queste persone della Cina rurale online sviluppano atteggiamenti riferiti non a fantasticherie ma all'amore filiale, al dovere, alla sincerità, alla saggezza, all'onestà, al corretto agire e al coraggio ${ }^{41}$, sperando di volta in volta che ciò possa influenzare positivamente il governo e la società. La famiglia è centrale nella moralità confuciana, e quindi usare i social media presentando il sé come incorporato nella vita familiare è importante. I post più popolari sono memi che celebrano l'amore e il matrimonio, foto di neonati e messaggi di status che ringraziano i propri genitori per la loro devozione e il loro supporto. Anche quando i messaggi di devozione filiale su QZone non vengono visti dai genitori, sono espressione della versione idealizzata della devozione nei loro confronti, messa in mostra per gli altri al fine di rappresentare il sé come persona morale. Questo genere di post popolari rivela un insieme di ideali che enfatizza il detto cinese bao xi, bubao you ("condividi la felicità, non le preoccupazioni”), che pertanto si appropria dei social media per riprodurre e rafforzare le cornici morali esistenti. 
L'esempio di McDonald riflette le scoperte di Miller nei suoi primi studi a Trinidad $^{42}$, nei quali concludeva che Facebook si comprendeva meglio non come uno spazio radicale di cambiamento sociale, ma come uno spazio di conservazione che si adopera per effettuare un ritorno ai valori tradizionali della comunità. Si tratta di un ideale piuttosto astratto. Un uso più originale dei social media riguarda il modo in cui le persone realmente trovano modi pratici di affrontare le domande tradizionali della comunità. Mentre i social media possono funzionare come uno strumento di nostalgia per il senso di comunità, possono anche agire come filtro quando la domanda di vera comunità sovrabbonda. Nell'etnografia della cittadina di Trinidad, Sinanan ${ }^{43}$ nota che le persone spesso si sentono oppresse dalle richieste senza fine fatte sui social dalla famiglia estesa e dagli amici. Spesso le persone si preoccupano che trascorrendo del tempo con una persona ne potrebbero offendere altre sei i cui inviti erano stati ignorati. A volte socializzare mediante i social media può completare la socializzazione di persona, consentendo di far spazio per più relazioni sociali.

Nel sito inglese molti individui, dopo essersi rimessi in contatto con compagni di scuola mediante i social media, poi si ricordavano del perché allora non avevano fatto in modo di restare in contatto. Ritenevano invece che $\mathrm{i}$ social media fossero un modo per recuperare queste persone, che stavano tra gli amici intimi e i conoscenti distanti, in una posizione che non era né troppo "calda" né troppo "fredda": la "Goldilocks Strategy". Restando connessi sui social, gli utenti erano in grado di dare l'apparenza di essere in contatto senza dover perdere tempo prezioso e energie per incontrarsi di persona. In questi casi i social media potevano essere molto efficaci per rafforzare ideali convenzionali, sia fornendo uno spazio per reiterare valori tradizionali sia offrendo nuovi meccanismi per alleviare impicci sociali di lunga data.

Insomma, per molti il tratto principale relativo al loro senso di felicità è la relazione con altre persone. Se i social media possono supportare le persone nel gestire gradi di socialità modulabile mediante le piattaforme che usano, allora questo può essere d'aiuto per tenere tutto in equilibrio e sotto controllo. Così, ci siamo spostati dall'idea che per le persone è importante una astratta relazione con i valori del passato all'osservazione che i social media possono davvero essere diventati una componente importante del modo in cui le persone fanno fronte agli obblighi tradizionali nel presente. 


\section{Piaceri temporanei}

Mentre le aspirazioni e i valori sociali di solito sono strutture di lunga durata che influenzano i dettagli della vita quotidiana, le persone possono anche fare esperienza della felicità in modo più fluido come una forma temporanea di piacere. Uno dei discorsi più comuni attorno ai social media in numerosi dei nostri siti, in particolare tra i più giovani, riguarda il fatto che i social media portano sì la felicità, ma solo di tipo effimero. Proprio come gli individui provano entusiasmo e piacere in tecnologie come i giochi elettronici, la musica, la televisione e i film, così i social media forniscono una fonte di intrattenimento che rende le persone felici, per un momento.

Per esempio, un giovane del nostro sito nella Cina rurale descriveva così a McDonald i giochi online: "Quando salgo di livello mi sento incredibilmente felice. Via via che il mio punteggio cresce sempre di più, e gradualmente va verso il top del livello, divento sempre più felice... ma non ci sono [diretti] benefici, solo la tua abilità è aumentata, in realtà non significa nulla, è solo un divertimento". È comune per i giovani in Cina cercare di aumentare il loro prestigio su QQ aggiungendo lingotti d'oro e gadget ai loro profili. Queste icone del prestigio si possono comprare utilizzando denaro digitale guadagnato semplicemente restando loggati e attivi. Nel nostro sito nella Cina industriale s'è trovato che alcuni operai spendono l'equivalente di un mese di paga acquistando auto digitali di lusso o un "titolo nobiliare su una piattaforma di social media come YY" ${ }^{4}$. Chiaramente spendere tanto tempo e denaro potrebbe indicare che questi individui sono felici durante le ore trascorse online. Quando però uno degli informatori di McDonald commenta: "in realtà non conta nulla", questa battuta indica che egli si gusta il piacere che arriva da giochi e social media in quanto individuo, ma deve anche essere deferente rispetto ai discorsi culturali più larghi e collettivi che sottolineano che "la vera felicità" è duratura; il piacere transitorio del gioco non può "contare" in questo gioco più grande della felicità.

Il coinvolgimento romantico è un'altra forma di felicità (a volte effimera) spesso associata all'uso dei social media. Nel nostro sito brasiliano tra i più importanti vantaggi dei social media ci sono le nuove occasioni di flirtare. Questo è positivo sia per le persone timide, che sono in grado di nascondere le loro insicurezze dietro lo schermo, sia per gli estroversi, che sono in grado di accedere a più persone e stabilire canali di comunicazione meno visibili per potenziali storie d'amore. Spyer scrive che Facebook è visto come una sorta di Disneyland per flirtare, ma molti individui nel suo sito raffigurano 
il flirt sui social media come una forma di piacere temporaneo e non come un tentativo di scoprire l'amore per la vita. Questi atteggiamenti riflettono la critica comune ad applicazioni come Tinder o Grindr come strumenti per relazioni superficiali, dominate da utenti in cerca di incontri sessuali occasionali e di una notte, e non di relazioni durature. Proprio come per il gioco, gli individui possono trovare piacevole un flirt online, ma loro stessi quasi sempre descrivono queste attività come superficiali e inferiori rispetto alle forme di felicità più durevoli.

Allo stesso tempo, mentre non sembra ancora esserci alcuna ricerca quantitativa sul tema, le persone in molti dei nostri siti indicavano che le applicazioni di incontri spesso portano realmente a relazioni durature e aiutano gli individui a superare ogni tipo di ostacolo, ansia o impedimento a costruire una relazione offline. I discorsi dominanti tuttavia continuano a bollarli come strumenti per incontri solo occasionali e carnali. Questo ci porta a una delle conclusioni più importanti di questo capitolo. Forse non possiamo imparare molto sulla questione se i social media portano felicità alle persone, ma esaminando i dettagli, stiamo imparando molto sulle concezioni di felicità e sui modi in cui le persone aderiscono a questi discorsi, per esempio la svalutazione della felicità transitoria, anche quando perseguono la felicità in modi che sono contrari alla retorica popolare.

\section{Social media e stress aggiuntivi}

Anche se il materiale della nostra inchiesta presta poco credito all'enfasi giornalistica sui social media come fonte di infelicità (nella fig. 4.26 i numeri che affermano che i social media rendono infelici sono decisamente bassi), resta ancora un elemento importante di questa questione. Mentre il nostro ultimo paragrafo rifiuta del tutto la pretesa di Tolstoj che "tutte le famiglie felici si assomigliano; ogni famiglia infelice lo è a modo suo", riteniamo ci sia tanta variazione nell'essere infelici quanta nell'essere felici.

I social media a volte presentano una visione bellissima della vita, e questo potrebbe aggiungere pressione perché altri la ottengano. Nella Cina industriale, specialmente fra gli abitanti locali di classe media, "conoscere la vita quotidiana di ciascuno" può portare gli individui a confrontarsi rispetto a ciò che fa sentire inferiori. Alcune persone riferiscono di sentirsi soli o depressi mentre altre riferiscono di un certo aumento dell'ansia, preoccupati per quello che 
potrebbero pensare gli altri che guardano il loro profilo sui social media ${ }^{45}$. In alcuni casi estremi, l'isolamento sui social media e il bullismo online possono avere impatti gravi. Un funzionario di fabbrica, Da Fei, nel sud della Cina descrive di essere stato escluso da un gruppo di WeChat dai suoi colleghi come colpo finale che l'ha portato a lasciare il posto di lavoro e la città. Questi effetti trovano eco in alcuni casi di bullismo tra compagni di scuola nel sito inglese. Le persone hanno sempre avuto la capacità di essere velenose e maligne, ma i social media potrebbero bene aver aumentato sia la capacità sia la gamma di possibilità per rendersi insopportabili l'un l'altra.

Spesso le persone che affermano che i social media le rendono meno felici tendono ad aver avuto qualche particolare esperienza negativa online, per esempio di stalking, molestie o un partner infedele. Nicolescu ${ }^{46}$ scrive che nel suo sito in Italia del sud gli avvocati concordano sul fatto che un aumento dei divorzi è provocato da Facebook e dal telefono cellulare perché i partner spesso scoprono le conversazioni inequivocabili che si svolgono tramite questi media. La visibilità di Facebook mette a rischio sia la coppia romantica sia la famiglia. Ne risulta che molte persone della regione che iniziano una relazione seria preferiscono chiudere l'account Facebook oppure aprire un account condiviso, che è considerato prova definitiva della serietà della relazione.

McDonald aggiunge che nella Cina rurale alcune donne controllano i telefoni dei mariti, cercando prove di infedeltà e parlano malissimo dei social media come una minaccia per il matrimonio. A Trinidad la sola differenza è che tale controllo dei telefoni funziona in entrambe le direzioni. Essere troppo visibile sui social media, o visibile nei modi sbagliati, può comportare il rischio che la propria intenzione venga fraintesa da altri e vengano gettate calunnie sopra la propria moralità. $\operatorname{Costa}^{47}$ riporta che le donne nella Turchia sudorientale ritengono che i social media possano contribuire all'aumento degli inganni parte dei mariti. Uno dei primi incontri di Costa nel campo di ricerca riguardò una donna che piangeva perché il suo ex marito l'aveva lasciata per andare a vivere con una donna più giovane che aveva incontrato su Facebook. Aveva preso i loro figli e non avrebbe consentito al suo partner precedente di incontrarli. Per le donne in Turchia e in Cina, proprio come per gli avvocati italiani, i social media sono spesso visti come la matrice dell'infedeltà - e quindi dell'infelicità - e non il mezzo mediante cui si manifesta. Se, da un lato, i telefoni cellulari e i social media rendono l’organizzazione di incontri privati più discreta, la possibilità che emergano 
prove fotografiche di questi incontri li rende più rischiosi. Un aumento della sorveglianza dei partner era evidente anche in Brasile e a Trinidad, dove i sospetti di infedeltà sono comuni, aggiungendo stress per entrambi i partner. In tutti questi casi stiamo riportando una percezione dei motivi e tutt'altro che una comparazione sistematica con le relazioni coniugali prima dei social media, dato che a quel tempo non c'eravamo. Potrebbe davvero non esserci alcun aumento nelle infedeltà o nei divorzi.

Ciò che possiamo dire è che la visibilità associata con i social media ha aggiunto stress da paura di infedeltà, ma ha anche contribuito al controllo in altri modi. Mentre le donne in Turchia sudorientale hanno guadagnato più grandi capacità di relazioni sociali come conseguenza dei social, sono sempre i media quelli con cui gli uomini le molestano con maggiore frequenza. Nei capitoli precedenti abbiamo anche visto lo stress che deriva dall'uso dei social media per il controllo sia da parte dello stato che della società. C'è più controllo politico e censura degli attivisti curdi, così come più controllo sociale su una scala intima effettuato dalla famiglia, da conoscenti, da amici.

Persino in contesti dove l'onore è meno preoccupante, i social media possono alimentare lo stress per come gli individui si presentano agli altri. Per esempio, nel suo studio in Italia, Nicolescu ha trovato che gli individui (in particolare le donne adulte) ritengono sia un peso creare un certo tipo di immagine positiva in pubblico. Ciò sta al centro di valori sociali locali e precede lo sviluppo dei social media, ma i social media diventano lo spazio dove tale pressione si avverte. Le donne hanno a lungo considerato necessario vestirsi bene quando escono, anche solo per andare in giro, e ora sentono la pressione nel costruire la loro auto-presentazione con cura quando caricano foto o quando fanno l'aggiornamento di status. Proprio come queste donne avvertono la responsabilità di rappresentare un ideale italiano di bellezza e di eleganza mediante i loro post su Facebook, l'élite istruita sente di dover mostrare le proprie credenziali intellettuali o artistiche online al fine di sostenere il proprio prestigio sociale.

In ognuno di questi casi i social media creano uno spazio per mostrare e ricostruire il capitale culturale in un'ambientazione in cui le persone sentono di dover avere una presenza pubblica che corrisponda alla loro posizione sociale. Questo, tuttavia, spesso è avvertito come un obbligo e non come un'opportunità. Un aumento generale nella visibilità procura non solo opportunità, ma anche ansia e un certo peso, comparabili almeno alla preoccupazione su cosa indossare in pubblico. 


\section{Discussione: i social media fanno apparire le persone più felici?}

Un risultato di questa pressione ad apparire online in modo appropriato è stata notata da Venkatraman nel suo sito in India del sud ${ }^{48}$. Egli osserva che le persone sembrano sempre felici su Facebook, proprio come riferiscono della pressione ad apparire felici in qualsiasi occasione pubblica. Con fotografie felici, messaggi positivi e anche frustrazioni espresse scherzando, la felicità non è in questo caso il prodotto di altre aspirazioni: è il vero oggetto dell'aspirazione stessa. Dato un contesto in cui media molteplici forniscono la scelta per diversi modi di comunicazione, conversazioni private tramite messaggi Facebook, WhatsApp o altre forme di messaggistica diadica possono convogliare più informazione sugli "autentici" stati emotivi, mentre Tweet o aggiornamenti di stato di Facebook più pubblici sono luoghi in cui gli individui con maggiore probabilità "mettono in mostra" la felicità al fine di costruire una particolare visione delle loro esistenze per il loro presunto pubblico $^{49}$. In India, la felicità è il valore sociale, e quando le forme pubbliche di social media proliferano diventano semplicemente nuovi spazi per esprimere un'apparenza di felicità.

I social media possono essere un luogo per riconnettersi o per sentirsi oltremodo connessi. Possono essere un luogo per esprimere vera felicità o per assumere, in modo ingannevole, un viso sorridente da mostrare. Forse questa ambiguità è il motivo per cui così tante persone rispondevano che i social media non fanno differenza rispetto alla loro felicità ${ }^{50}$ (una percentuale del 56.68 per cento in tutti e nove i siti di ricerca). Allora, anche se riconosciamo il problema di definire la felicità, c'è qualche vantaggio ad aver posto la domanda in questo capitolo perché i social media hanno creato un enorme spazio in più dentro cui le persone presentano e ripresentano felicità ed emozioni. Abbiamo anche visto come questo spazio regola l'equilibrio fra il visuale, l'aumento dell'accesso alle informazioni, lo sviluppo della capacità di avere aspirazioni a questo associato e, in certi casi, per esempio nella Cina industriale, la possibilità di soddisfare queste aspirazioni. Così, il valore di chiedersi se i social media abbiano un impatto sulla felicità potrebbe consistere non tanto nell'imparare se le persone sono più felici per via dei social media, quanto piuttosto nell'apprendere come possiamo usare i social media per capire di più cosa la felicità significhi per i loro utenti, e come e dove lo esprimono. 



\section{Capitolo 14 \\ Il futuro}

Forse la principale ragione per cui gli antropologi sono cauti nel lanciarsi in predizioni sta nel fatto che nello studio del presente vediamo anche il destino delle predizioni del passato. Più di questo, comprendiamo perché esse siano raramente significative. La vita quotidiana come viene osservata in etnografia con la sua contestualizzazione olistica è molto più complessa che un laboratorio in cui si possono controllare le variabili. Apprezziamo anche che una predizione spesso è altamente motivata. Data la natura aperta dei mercati moderni, ci sono molte persone che guadagnano dalla corretta valutazione di ciò che accadrà.

Il timore di contribuire a tale frenesia non dovrebbe però impedirci di esplorare altre istanze rispetto al futuro. Potremmo ancora redigere documenti contenenti linee guida per un ospizio suggerendo come potrebbero usare i social media nel futuro ${ }^{1}$, o programmare di rifinire i nostri metodi di studio in antropologia per prendere in considerazione l'impatto dei new media. Con una certa ragionevolezza ci assumiamo la responsabilità di aiutare le persone a considerare le conseguenze dei social media in modi che potrebbero nel futuro rilanciare, e non deprimere, il loro benessere, per esempio fare disposizioni anticipate riguardo a ciò che accadrà ai loro materiali online alla loro morte. Così, ci sono molte buone ragioni per tentare almeno di usare le evidenze del presente per intravedere che cosà potrebbe accadere in seguito.

La "teoria del raggiungimento" ${ }^{2}$, che è stata sviluppata precedentemente a questo progetto al fine di teorizzare l'impatto delle tecnologie dei new media sulla nostra comprensione dell'umanità, sostiene che, tipicamente, i new media all'inizio sono usati in modo conservativo, per ottenere qualcosa che si desidera già, ma più facile da ottenere con il loro l'aiuto. Per esempio, i social media si usano per porre rimedio alla frattura cui vanno incontro le famiglie transnazionali divise o per andare incontro al desiderio, prima insoddisfatt, di condividere più facilmente le fotografie. Presto, però, le cose si muovono verso nuovi ambiti. Nel 2016 l'enfasi sull'uso dei social media non sta più nella possibilità di ricucire contatti persi (come in Friends Reunited) o nel realizzare la fantasia di connessioni del tutto nuove. Ciò che invece osserviamo oggi rivela 
qualcosa di più simile a un raffinamento. Considerati alla luce della nostra teoria della socialità modulabile, i piccoli gruppi di WhatsApp vengono ora usati per equilibrare i più grandi gruppi di Facebook. L'intimità di Snapchat bilancia il contatto fra estranei di Twitter o Instagram. Le console di gioco online come Xbox e Playstation, insieme ai giochi dei social media che hanno fatto seguito a Farmville, ora bilanciano il precedente isolamento del singolo individuo che gioca con nuove possibilità sociali. La socialità modulabile non è solo l'osservazione di una configurazione che si dipana attraverso i social media, ma anche una previsione circa il fatto che le nuove piattaforme colonizzeranno altri spazi lungo queste scale.

I nuovi media raggiungono costantemente nuove possibilità. In relazione al tempo, ci sono opportunità maggiori sia per la comunicazione simultanea sia per varie versioni di conversazione asincrona. In relazione allo spazio, osserviamo la "fine della distanza" arricchita da nuove possibilità di una "specie di co-presenza", per esempio coppie che vivono in nazioni differenti che vivono una "specie di" vita insieme online; tali possibilità si possono analizzare più a fondo nello studio della webcam ${ }^{3}$. I dispositivi digitali inoltre modificano il nostro senso della memoria collettiva ${ }^{4}$, creando una nuova forma di combinazione di facoltà interne e esterne per raccogliere informazioni. Non ci prendiamo più tanto gioco dell'online come una seconda mente 5 Assistiamo a una radicale espansione non solo in fotografia e nella comunicazione visiva $^{6}$, ma anche, forse più marcatamente, nella nostra capacità di condividere. La socialità modulabile è, per questo, solo uno dei molti modi in cui il nostro progetto riconosce che i social media espandono la nostra capacità ma, ribadiamo, non cambiano la nostra umanità essenziale ${ }^{7}$. Il punto della teoria del raggiungimento è che, in nessun caso, è in questione né una perdita di umanità né una condizione possibile di post-umanità.

\section{Quattro tendenze}

Entro il vasto ambito di conseguenze dei nuovi social media, come antropologi la nostra ricerca focalizza selettivamente le loro implicazioni per la socialità. Entro questo più stretto compito, questa sezione esaminerà quattro tendenze. La prima è già chiara come socialità modulabile: un crescente numero di piattaforme di social media può essere messo in linea con la diversità dei gruppi sociali ai quali desideriamo collegarci. Sembra esserci 
un'accelerazione dello sviluppo di nuove piattaforme, probabilmente perché, come sembra essere stato il caso di WhatsApp, i costi dello sviluppo di nuove piattaforme start-up sono crollati, mentre il tempo che ci vuole perché si consolidino si è contratto. Ne risulta anche un minor impedimento a che nuovi dispositivi e nuove piattaforme occupino piccole nicchie in attesa di essere sfruttate. La sottile sintonia di questo equilibrio fra comunicazione privata e pubblica sta diventando cruciale per il modo in cui le persone, nel nostro sito brasiliano e in quello italiano per esempio, fanno esperienza dei social media.

Nel Capitolo 1 è stato indicato che la socialità modulabile dovrebbe includere anche i media precedenti, per esempio le chiamate telefoniche diadiche e la trasmissione pubblica. E possibile che via via che viene coperto maggiormente, sarà più difficile individuare lungo questo spettro un gruppo particolare che possa essere isolato come "social media". In breve, una conseguenza di questa previsione è che la stessa idea di "social media" potrebbe gradualmente sparire; invece, abbiamo semplicemente un insieme di media sempre più diversi e uno sfruttamento delle possibilità che questi media hanno creato sempre più sofisticato, e anche altre tendenze come: ottenere informazioni, condividere informazioni o rendere la comunicazione più visiva. Tutti i media sono sempre stati sociali, e la separazione di un gruppo di essi definito come social all'interno dello spettro generale potrebbe, nel tempo, diventare meno utile.

La previsione che la conclusione del successo dei social media possa consistere nel loro non essere più una sfera separata indica inoltre un altro importante sviluppo tecnologico: lo smartphone. Per quanto sia diventato potente e importante l'avvento dei social media in alcuni dei nostri siti, sarebbe difficile metterlo davanti all'impatto e al significato degli smartphone, entro cui le piattaforme di social media potrebbero spesso essere visti solo come un altro tipo di $a p p^{8}$. Per di più, sono gli smartphone che consentono l'importanza dei social media come un insieme di multimedialità, rendendo chiaro l'ambito di possibilità dei media in quanto posti fianco a fianco dentro un dispositivo facilmente accessibile. Le persone non possono soltanto scegliere la scala del gruppo che vogliono per comunicare, ma anche l'insieme di componenti testuali, visive e uditive appropriate alla situazione. Come si può notare con Snapchat o l'uso di Facebook da parte di popolazioni analfabete in India meridionale, è possibile che una "conversazione" sia ora quasi interamente visiva. Man mano che gli smartphone diventano più smart, 
possono ben accelerare la dissoluzione dei social media in questo più ampio schieramento di possibilità comunicative. Questo insieme fluido di forme comunicative si adatta al modo in cui gli utenti fluttuano fra attività come parlare, giocare, comporre testi, masturbarsi, imparare, fare acquisti.

Con una maggiore enfasi sullo smartphone non sono solo i social media che possono diventare più integrati, ma anche le stesse piattaforme individuali. Al momento è consueto discutere di social media in termini di QQ o Facebook, Twitter o Instagram. Però fino a poco tempo fa la messaggistica istantanea sarebbe stata intesa anche in termini di piattaforme come AIM, MSM o BB. Oggi, tuttavia, un messaggio di testo può ugualmente arrivare tramite WhatsApp, WeChat, il dispositivo per messaggiare proprio dello smartphone, un messaggio privato di Facebook o un'app come Viber. Le persone stanno diventando sempre meno attente a quale di questi sia. $\mathrm{La}$ tendenza è, per questo, verso un contenuto che trascende la piattaforma dalla quale è comunicato, e questo potrebbe certamente continuare. Inoltre, questo progetto prova che queste piattaforme non sono solo dispositivi tecnici, ma che con esse si ha anche un rapporto come con una "sorta di" amico. QQ è stato presente per un periodo più lungo rispetto alla maggior parte di altre piattaforme di social media; un tempo considerato come nuovo e entusiasmante, ora è percepito da molti un po' come un parente rassicurante ma anziano. A giudicare dalla perdita di attrattiva fra i giovani in Inghilterra, Facebook potrebbe aver iniziato ad andare allo stesso modo. I giovani in cerca di nuove esperienze e nuovi spazi possono creare una dinamica nei social media che non ha niente a che fare con la funzionalità e ha invece a che vedere con la moda e con le loro relazioni tra pari. Forse potremo continuare a usare piattaforme come marchi di fabbrica che stanno a indicarne la funzione, come gli inglesi facevano con Hoover (per aspirapolvere), Biro (per penna a sfera) o Sellotape (per nastro adesivo). Potremmo continuare a parlare di twittare anche quando Twitter non esisterà più. Una chiara conclusione di questo progetto è che le piattaforme contano molto meno di quanto una volta pensassimo.

Entrambe queste tendenze sono strettamente legate a una terza molto chiara predizione, il movimento verso l'ubiquità. Un libro eccellente sulla nascita dei new media si intitola The Great Indian Phone Book'. Benché non centrato particolarmente sui social media, presenta una rassegna ben documentata di ciò che sembra stare accadendo nel momento in cui la grande popolazione dell'India si compone di proprietari di telefono. Carla Wallis 
ha scritto un libro simile con riferimento alla Cina ${ }^{10}$. Insieme, queste aree coprono metà della popolazione mondiale. Questi libri dimostrano che non avremo da attendere fino all'eliminazione della povertà per vedere la possibile ubiquità dei social media basati sul telefono. Questo è in parte dovuto al fatto che i prezzi di tali dispositivi continuano a abbassarsi in conseguenza della produzione di massa cinese di smartphone di alta qualità, ma anche al senso priorità che tali dispositivi rappresentano per i relativamente poveri. Tra gli informatori nella cittadina industriale cinese, il 91 per cento ora accede a internet tramite questo tipo di smartphone ${ }^{11}$.

La crescita più veloce della popolazione globale è in Africa. Qui Kenya e Sudafrica insieme sono avanti, e gli esperti più in generale prevedono una crescita nel possesso di smartphone estremamente rapida ${ }^{12}$. Nel complesso, The Economist ${ }^{13}$ prevede che l' 80 per cento della popolazione mondiale avrà smartphone decentemente connessi entro il 2020, sottolineando che le dieci magiori app di messaggistica rappresentano già tre bilioni di utenti. Né si tratta di semplice possesso. Le persone trascorrono diverse ore al giorno su questi dispositivi. Questo si potrebbe rappresentare come la fine del digital divide. E ancora, mentre molte persone nel mondo avranno accesso a parte rilevante della tecnologia, il nostro capitolo sulla disuguaglianza indica che la parità di condizioni di accesso ai dispositivi può avere un impatto molto limitato sulla più ampia disuguaglianza sociale. Inoltre, come sostiene Mark Graham che lavora sui media africani, la presenza di un uso africano di internet consistente non significa necessariamente che su Wikipedia o Google le questioni africane saranno automaticamente più presenti $\mathrm{i}^{14}$.

Una delle spinte trainanti del nostro progetto risiede nel fatto che i precedenti studi hanno in gran parte mancato l'obiettivo di seguire l'espansione globale dei social media. Anche se in futuro i dispositivi potranno essere presenti in ogni angolo del globo, resterà la disuguaglianza in termini di servizi disponibili in certi posti e di mancanza di attenzione alle esigenze e ai desideri di determinate popolazioni. Nello stesso tempo, riconoscere che questo può non necessariamente avere effetti su qualsiasi altro aspetto della disuguaglianza non dovrebbe impedirci di riconoscere che, sotto un certo aspetto, esiste una crescente e significativa parità: il semplice fatto di avere uno smartphone consente un cambiamento significativo nelle capacità di chi lo possiede.

In un movimento simile verso l'onnipresenza, i social media non stanno solo diventando indispensabili alla popolazione dispersa dal punto di vista 
geografico, ma anche rispetto a una sfera anagrafica più frammentata. Il conservatorismo relativo ai social media è stato fino a poco tempo fa per lo più associato all'età. All'inizio del nostro progetto, a metà del 2012, molte persone presumevano che i social media avessero una sorta di affinità naturale con i giovani, soprattutto perché raggiungere i giovani era stata la priorità, almeno idealmente, di tutte le aziende commerciali che avevano creato piattaforme di social media. In un certo senso, però, c’è una relazione inversamente proporzionale fra queste forze commerciali e ciò che gli antropologi hanno precedentemente osservato in molti paesi, ossia il fatto che la comunicazione sociale come attività è molto più comunemente associata alle donne più anziane, che non ai giovani maschi. Sono più spesso le donne d'età a svolgere il lavoro collettivo di tenere insieme la comunità e le norme sociali tramite ciò che viene definito, in modo dispregiativo, "pettegolezzo". In molti dei nostri siti rimaneva una qualche divisione generazionale, con un uso ancora molto ridotto da parte delle persone più anziane tra le popolazioni del Brasile rurale, della Cina e dell'India. Ciò tuttavia si sta erodendo, e sembra molto meno vero se riferito alla diffusione degli smartphone che non alle piattaforme di social media. Come indicato, la pervasività crescente dello smartphone catalizza un uso più generale dei social media. Le persone più grandi in alcuni dei nostri siti di ricerca sembravano usare con scioltezza media visivi come Skype o Facetime e tablet come iPad. Nella misura in cui i social media restano associati con il nocciolo duro della comunicazione sociale, tipo il pettegolezzo, il futuro dei social media potrebbe davvero fondarsi più sulle donne anziane che sugli uomini giovani (si pensi alla linea fissa).

Giustapporre queste tre tendenze separate entro il contesto dei nostri risultati di campo, ci porta a una quarta predizione che è molto meno ovvia intuitivamente, ma che emerge dalle nostre prove etnografiche. Come suggerito nei capitoli di questo volume dedicati al genere, alla politica e alla disuguaglianza, i social media esercitano il loro impatto più profondo sulle popolazioni che sono state tradizionalmente le più costrette. Anche se per nessuna popolazione i social media sono né del tutto positivi né del tutto negativi, sembra davvero che abbiano effetti emancipativi per molte popolazioni marginalizzate. Forse il miglior esempio di trasformazione radicale è il lavoro di Costa tra le donne a Mardin, le quali usano i social media per superare rigidi vincoli alla loro libertà di comunicare con gli altri; questo tipo di pratica inoltre richiama scoperte fatte altrove, in Medioriente, 
sull'impatto dei social media in paesi come l'Arabia Saudita ${ }^{15}$. Il punto è davvero piuttosto semplice: più gli individui vivono all'interno di vincoli sulla comunicazione imposti culturalmente, più una nuova tecnologia può significare che ciò che prima era vietato ora diventa possibile. Lo studio di Costa mostra che l'impatto cambia radicalmente le relazioni sociali fra uomini e donne e, potenzialmente, la posizione generale delle donne all'interno della società. Niente di questo è contraddetto dalla constatazione che i social media sono ugualmente e nella medesima società di gran lunga più conservatori di quanto ritenessimo in passato.

\section{Sia l'uno che l'altro, nello stesso tempo}

Mentre alcune di queste tendenze potrebbero basarsi su sviluppi tecnici attuali e futuri, come rivelano tutti i siti di questo progetto, la scelta di un preciso social medium in un ambiente polimediale più che sulle affordance tecnologiche si basa sui generi locali di interazione sociale o di costruzione del significato culturale. In Inghilterra, le persone più grandi erano molto soddisfatte dalle affordance tecnologiche offerte dalla diffusione di Facebook. Se i giovani, tuttavia, smettessero di postare su Facebook e i più grandi volessero mantenere i legami con i giovani potrebbero essere costretti a seguire i giovani su una piattaforma meno agevole: la connessione sociale è più importante di quanto una piattaforma risponda alle loro esigenze in modo soddisfacente.

Analogamente, in Cina la configurazione dell'uso differenziale delle piattaforme piuttosto che mostrare semplicemente ciò che di buono si può con fare una specifica piattaforma tende a riflettere la consapevolezza delle classi emergenti e la precedente separazione urbano-rurale. Quando gli operari cinesi acquisiscono competenza in una piattaforma come QQ, possono cercare siti più nuovi che li aiutino a restare moderni nel confronto con gli altri. Nel sito della Cina rurale, McDonald indica come la migrazione alle aree urbane per brevi periodi di lavoro o di studio spesso portava le persone a iniziare a usare WeChat per comunicare con i nuovi contatti presi in questi posti.

Il desiderio di emulare regioni più metropolitane è forte in alcune aree che si considerano periferiche, per esempio nel nostro sito nell'Italia del sud, dove le persone di più basso capitale culturale e d'istruzione tentano di emu- 
lare quelle che ne hanno di più. Tuttavia, come sempre, nel nostro progetto ci sono eccezioni, laddove le persone non usano i social media per esprimere la distinzione sociale in questo modo. Nell'analisi di Haynes del suo sito in Cile una questione centrale riguarda il fatto certo che la città crea la propria identità esattamente nel rifiuto di emulare, e persino di rispettare, la regione metropolitana rappresentata dalla capitale Santiago. Ad Alto Hospicio le persone spesso si riferiscono all'area come "Santi-asco", dove "asco" è il termine spagnolo per "disgusto".

Così, nell'individuare quale medium usano le persone, si possono fare generalizzazioni sull'importanza permanente dello status sociale e dell'emulazione di contro alla mera affordance tecnica, ma non si possono fare semplici previsioni su un qualsiasi esito di tutto questo. Mentre qualcuno potrebbe aspettarsi una tendenza, sul lungo termine, a una maggiore e più efficiente convergenza tra affordance ed esigenze, è del tutto improbabile stabilire se fattori come la moda e l'emulazione continueranno a rivelarsi più potenti della funzionalità.

Dopo tutto, non ogni cosa cambia nel tempo per diventare più funzionale o efficiente. Della maggior parte dei vestiti o del cibo non diremmo che sono oggi più efficienti rispetto a diversi secoli fa: piuttosto sono aumentati nella diversità per ragioni legate alle mode e alla differenziazione sociale. Al contrario, l'abbigliamento funzionale, come anche il cibo salutista, sono esempi ricorrenti di stili di vita. Così, stiamo dicendo semplicemente che non ci siamo ancora resi conto del fatto che i social media possono essere più come i vestiti o il cibo. Ciò che i nostri studi scoprono come relativamente solido e duraturo sono non le piattaforme di social media che le persone usano, quanto piuttosto i generi di comportamento configurati culturalmente messi in scena su e attraverso questi siti. I modi in cui i cinesi socializzano sul cibo, gli inglesi al pub, gli abitanti di Trinidad a un party, gli indiani attorno alla famiglia estesa o gli italiani nello spazio pubblico sono quasi sempre riprodotti sui social media. Qui, così come in molte altre parti del mondo, i social media rappresentano un cambiamento del luogo dove le cose accadono, non di ciò che accade.

Per questi motivi gli antropologi si aspetterebbero che gli sviluppi futuri su queste piattaforme includano anche un adattamento e un'affinità maggiori rispetto ai generi culturali locali. Come indicato nella discussione sul lavoro e il commercio nel Capitolo 5, le questioni finanziarie sono viste come opposte alla vita intima e personale in Europa, ma come un'espressione di intimità in Cina. Di conseguenza, Amazon rappresenta una ulte- 
riore de-socializzazione del commercio se comparata al fare acquisti offline: nel fare le transazioni abbiamo anche meno connessioni con le persone reali $^{16}$. Tuttavia, Taobao, l'equivalente cinese per lo shopping, rappresenta una ri-socializzazione della vita economica, un posto dove le persone costruiscono legami personali. McDonald indica che, almeno nella sua cittadina della Cina rurale, usare i social media per fare acquisti è visto come una parte integrante della vita familiare; è un'attività morale in sé e per sé. Mentre nell'area nord-atlantica a sostenere il commercio sono per lo più le aziende come Facebook, nel nostro sito cileno una quantità crescente di transazioni commerciali è gestita su Facebook da individui. Essi creano gruppi come "Compra e vendi di tutto", che funzionano come un forum online sia per postare oggetti in vendita sia per richiederne. Sarebbe però un fraintendimento considerarla solo come una forma di commercio. In questo genere di gruppi, come nel nostro sito italiano, il commercio di solito apre una finestra sulla vita sociale tanto quanto sul guadagno di denaro.

Lo studio delle affordance tecniche ha forse un potenziale maggiore per formulare previsioni qualora venga considerato in possibile convergenza con le preferenze culturali. Il Brasile, per esempio, è stato dominato da Orkut, che legava gli individui non solo a chiunque già conoscessero, ma anche a coloro con i quali si aveva già molto in comune, anche se non ancora parte del proprio network sociale. Questo andava incontro a una sensibilità brasiliana per cui i social media dovrebbero essere un posto dove "sta chiunque". Benché per molti aspetti simile a Facebook, Orkut era sganciato da qualsiasi servizio di messaggistica. Dato questo allargamento all'inclusione di nuovi amici che erano estranei, le persone volevano tuttavia avere la certezza di poter svolgere conversazioni private. Ciò ha portato prima a un passaggio a Facebook e poi, a sua volta, a WhatsApp, che sembravano più brasiliani perché incorporavano questi tratti. In un certo senso, quindi, i social media aiutano i brasiliani a diventare anche più brasiliani di quanto non fossero stati in grado di esserlo nel passato. Come nella nostra teoria del raggiungimento, uno studio della vita sociale precedente rivelerebbe un desiderio latente di essere legati agli altri in questa maniera complessa che comprende sia una dimensione privata sia una pubblica. Tale desiderio è soddisfatto più pienamente grazie ai social media. Analogamente, i social media consentono ai brasiliani di sentirsi più moderni, negando la sensazione di essere arretrati e rurali, e ottenendo questo senza dover rompere i loro contatti con le famiglie e i posti di origine. Qui non siamo discutendo dunque di alcune 
tendenze generali verso una funzionalità più efficace, ma dello sfruttamento di quelle potenzialità dei social media che soddisfano un modello di socializzazione locale, sintonizzato culturalmente.

Queste previsioni inoltre spiegano perché spesso la risposta a molte delle domande poste in questo volume è "né ' $\mathrm{X}$ ' né ' $\mathrm{Y}$ ', ma l'uno e l'altro allo stesso tempo". Le teorie della cultura, al contrario delle discussioni comuni, tendono a non enfatizzare tendenze in una qualsivoglia direzione, piuttosto a riconoscere le contraddizioni ${ }^{17}$. Abbiamo mostrato che i social media rendono il mondo sia più omogeneo globalmente sia più eterogeneo localmente, più individuale e più sociale, più uguale e più disuguale, più liberale e più conservatore, aumentando sia la mercificazione sia la de-mercificazione. Il futuro è solitamente di più di tutt'e due. Per esempio, a Trinidad l'affermarsi del telefono cellulare individuale può davvero aver agevolato le relazioni sessuali segrete, dato che il contatto diretto era più facile che in precedenza; tuttavia, l'essere taggato in una fotografia su Facebook insieme al partner sbagliato rende molto più difficile nascondere una relazione. WhatsApp offre più spazio per la privacy, ma molte persone sarebbero colpite dal vedere le loro erranze quotidiane registrate fedelmente tramite la cronologia dei movimenti su Google. Riscontriamo maggiori minacce per la privacy, come evidenziato da Edward Snowden e Wikileaks, ma anche maggiore capacità di mantenere la privacy e l'anonimato, come nel resoconto di Costa sulle relazioni intime a Mardin e come nella prime esperienze di privacy di alcune persone in Cina. Così, la migliore risposta a molte domande sul futuro impatto dei social media sul comportamento sociale è "sia l'uno che l'altro, nello stesso tempo", dove ciò equivale allo sviluppo di tendenze contrastanti ${ }^{18}$.

\section{Immaginare il futuro}

Una delle cose che possono essere cambiate durante lo svolgimento del nostro lavoro sul campo fra il 2013 e il 2014 riguarda la relazione fra social media e concettualizzazione del futuro. La nuova tecnologia non si limita a cambiare il modo in cui le persone si comportano nelle loro vite quotidiane: essa facilita anche la nostra immaginazione del futuro. C'è spesso un legame implicito fra i discorsi sul futuro e le idee di tecnologia, per cui se guardiamo un programma televisivo con un titolo come Il mondo di domani ci aspettiamo che l'argomento riguardi la tecnologia. 
Anche se non fa parte del nostro studio, abbiamo chiaro che lo sviluppo dei social media in quanto nuova tecnologia dipende da persone che trascorrono una grande quantità di tempo a pensare al futuro e a interrogarsi su esso e a come la tecnologia possa aiutarli a realizzarlo. Malaby ha passato in rassegna le varie forme di ideali tecno-liberazionisti spesso utopistici ${ }^{19}$, importanti nello sviluppo di alcune piattaforme digitali come Second Life. Questi includono gli ideali più anarchici trovati da Coleman nel suo studio sulle comunità di hacker ${ }^{20}$, o lo studio di Kelty sugli idealisti che assumono a modello per la sfera pubblica del futuro lo sviluppo della codificazione Open Source ${ }^{21}$. Anche se questi gruppi sono relativamente piccoli e specifici, parti più ampie della popolazione vi partecipano indirettamente mediante attività come le campagne di Crowdsourcing e Kickstarter o di diffusione di Open Access publishing. Tutti questi parlano a un nuovo futuro immaginato che tende all'idealismo. Anche se niente di ciò ricade nel nostro studio, questo libro presenta molti esempi di come i social media si siano erti a segno di aspirazione e di modernità, spiegando il passaggio da QQ a WeChat in Cina o il declino dello status di Facebook per i giovani.

Per le popolazioni occidentali, per molti anni, grazie allo sviluppo incrementale di hardware come il pc e software come Windows Office, si era manifestata una visione verosimilmente autentica di un futuro sempre in espansione. Per più di una decade, ogni anno sembrava portare con sé nuovi modelli e miglioramenti. Tuttavia, si comprese anche che non si trattava di un processo infinito: una volta raggiunto un certo livello, avere un PC o una versione di Office ancora migliore da motivo di intenso entusiasmo si trasformò in qualcosa quasi privo di interesse. Windows ha appena annunciato che il proprio sistema operativo non avanzerà oltre il numero dieci. L'attenzione si sposta invece verso qualcos'altro, come l'ultimo tablet. Per le persone di paesi a basso reddito l'equivalente di tutto ciò è stato l'arrivo annuale di nuovi smartphone, e la loro anticipazione dei social media e il loro incontro con gli stessi. Per un po' potrebbe essere sembrato che i social media avessero terminato la loro evoluzione con la pervasività di Facebook e QQ. Tuttavia, gli ultimi due anni hanno mostrato un nuovo movimento verso la multimedialità e piattaforme come Tinder o Snapchat o WeChat sono cresciute in popolarità; tutto ciò ci aiuta a mantenere il legame fra nuovi social media e anticipazione degli sviluppi futuri.

Così come l'idea che l'ultimo computer esprima il futuro si è oggi attenuata, è anche probabile che il ruolo dei social media come dimensione 
simbolica del futuro possa già essere in declino. Al contrario, come con tutte le tecnologie digitali e come sostenuto da Miller e Horst ${ }^{22}$, il punto più sorprendente di queste nuove tecnologie riguarda il fatto che dall'essere emblematiche di un futuro quasi irraggiungibile possono facilmente diventare così scontate che quando non funzionano sembra quasi un dispetto personale. Questo processo può richiedere solo qualche mese o anche qualche settimana. Forse il primo esempio di questo durante il periodo di campo sono stati WhatsApp e WeChat. Uno dei nostri primissimi blogpost per questo progetto è stata una nota sul fatto che a Trinidad le persone stavano adottando WhatsApp in un momento in cui in Inghilterra era quasi sconosciuto $^{23}$. Quindici mesi dopo, quando il nostro fieldwork era finito, WhatsApp era chiaramente diventato una delle tante piattaforme globali e ubique; date ora del tutto per scontate, facciamo fatica a immaginare che prima non ci fossero.

Nel modo in cui la tecnologia in se stessa diventa simbolo di modernità risiede una delle ragioni per cui essa diviene espressione di valori culturali, piuttosto che esserne distinta ${ }^{24}$. Anche questo, tuttavia, dipende dallo specifico senso culturale di cosa significhi diventare moderni, proprio di ciascun sito. Come discusso nel volume sulla Cina industriale ${ }^{25}$, una storia "umiliante" - che, in termini comparativi, rappresenta una mancanza di sviluppo tecnologico nella Cina moderna per gran parte del Ventesimo secolo - è ciò che ha portato l'intero paese, dall'élite ai contadini, a vedere la nuova tecnologia come simbolo del revival nazionale. Ciò ha portato a investimenti governativi considerevoli, e realmente gran parte della popolazione condivide il focus del governo su questa impresa. Di conseguenza, è meno probabile trovare in Cina il tipo di resistenza digitale notato da Sinanan nel suo sito a Trinidad, dove il rifiuto di accogliere nuova tecnologia mediatica può essere una sorta di ostentazione ${ }^{26}$, anche se ci sono atteggiamenti equivalenti anche nel sito della Cina rurale. Questo ha comportato anche risultati a balzi. In Cina, per esempio, le persone stavano già archiviando tutti i loro contenuti online $^{27}$ prima dello sviluppo dell'archiviazione cloud in Occidente; non avevano preso parte alla fase precedente di sviluppo e così non avevano nessuno degli atteggiamenti conservatori derivanti da precedenti attaccamenti. 


\section{Conclusioni}

È ovviamente problematico prevedere il futuro per qualcosa di così dinamico come i social media. La sola previsione che riteniamo possibile fare è che ciò che prevediamo si rivelerà errato. L'aspetto più importante di questo libro tuttavia riguarda il riconoscimento del fatto che prevedere il futuro è, in qualche modo, difficile quanto conoscere il presente. Lo scopo di questo nostro studio è stato, soprattutto, argomentare in che modo molte delle nostre assunzioni rispetto a ciò che riteniamo di conoscere già, di fatto, sono sospette. Nel momento in cui comprendiamo che conoscere i social media non riguardi tanto la descrizione di un insieme di piattaforme, quanto piuttosto riconoscere cosa il mondo ci ha messo dentro, va da sé che l'enormità della questione è già palese. Come possiamo sapere che cosa sono diventati i social media per i lavoratori nei pozzi petroliferi in Alaska, per i popoli tribali in Amazzonia e per i nuovi ricchi a Mosca?

Ovviamente non possiamo sapere che cosa siano davvero i social media, rispetto a un ideale di conoscenza totalizzante, ma possiamo almeno assumere contezza di quanto non sappiamo. Dando ugual peso ai nove siti sparsi nel mondo, speriamo che con questo libro abbiamo anche dato un contributo per chiarire quali forme di conoscenza sono ancora possibili. Possiamo dare il senso della creatività di più ampi settori della popolazione come anche dei fanatici della tecnologia (geeks) nella produzione dei social media che concretamente usiamo. Possiamo mostrare in che modo l'obiettivo di comprendere i social media ci abbia a sua volta regalato ritratti ravvicinati delle vite dei nostri contemporanei. Abbiamo mostrato in che modo l'antropologia risulti complementare alle molte altre discipline che studiano i social media, ognuna delle quali da un propria prospettiva. Infine, abbiamo cercato di mostrare in che modo l'antropologia comparativa produca particolari aspetti della conoscenza sia in ampiezza sia in profondità. A rendere questo essenziale, nel contesto della complessità nostro mondo moderno, è tuttavia il fatto che queste forme di comprensione si basano sull'empatia. 



\section{Note}

\section{Capitolo 1}

1 Nella versione inglese del volume gli autori hanno usato il singolare per riferirsi ai social media in generale e il plurale se il riferimento era specifico a social media come un ambito di piattaforme. Nella traduzione questo è stato modificato in base alle regole della lingua italiana $[\mathrm{NdT}]$.

2 Incluso D. Miller, parte del nostro gruppo di ricerca. Miller, D. 2011. Tales from Facebook. Cambridge: Polity Press.

3 Anche se in alcune aree WhatsApp spesso si usa per inviare messaggi vocali.

4 Lo studio della socialità ha una lunga storia. Il suo fondatore forse è stato il sociologo Georg Simmel, che agli inizi del Novecento tentò di elaborare le differenti caratteristiche delle diadi di due, le triadi di tre e le qualità più grandi di ciò che definiamo società o comunità. Si veda Simmel, G. Wol, K.H. 1950. The Sociology of Georg Simmel. Glencoe, IL: Free Press.

5 Madianou, M., Miller, D. 2012. Migration and New Media. London: Routledge.

6 Si veda anche Marwick, A., boyd, d. 2014. «Networked privacy: How teenagers negotiate context in social media». New Media \& Society, 16(7): 1051-1067.

7 Il materiale di comparazione è disponibile nei dettagli nelle rispettive nove monografie e anche in un altro volume della serie intitolato Visualising Facebook, che compara in modo sistematico i post visivi in Inghilterra e a Trinidad.

8 Miller, D., Sinanan, J. 2014. Webcam. Cambridge: Polity Press. Capitolo uno.

9 Goffman, E. 1959. The Presentation of the Self in Everyday Life. Garden City, NY: Anchor Books.

\section{Capitolo 2}

1 La scelta di tenere il nome minuscolo è dell'autrice

2 Non significa ignorare decenni di lavoro precedente sulla comunicazione mediata dal computer, ma semplicemente riconoscere il momento in cui tale lavoro sembrò realmente il punto di partenza per lo studio specifico dei siti di social networking.

3 boyd, d., Ellison, N.B. 2007. «Social Network Sites: Definition, History and Scholarship», Journal of Computer-Mediated Communication, 13(1): 211-230.

4 boyd, d. 2014. It's Complicated: The Social Lives of Networked Teens. New Haven, CT; London: Yale University Press: 6-14.

5 Quando l'espressione "siti di social networking" prevaleva c'era forse anche un focus 
maggiore su approcci riguardanti il networking, per esempio Papacharissi, Z., ed. 2011. A Networked Self. London: Routledge.

6 boyd, d., Ellison, N.B. 2007. «Social Network Sites: Definition, History and Scholarship», Journal of Computer-Mediated Communication 13(1): 211-230.

7 Weller, K. et al., eds. 2013. Twitter and Society. New York: Peter Lang. Si vedano in particolare i saggi di Rodgers, Schmidt, Bruns e Moe e di Halavais.

8 Non abbiamo la tentazione di definirli "network privati".

9 Van Dijck, J. 2013. The Culture of Connectivity. Oxford: Oxford University Press.

10 Per esempio Graham, M., Dutton, W., eds. 2014. Society and the Internet. Oxford: Oxford University Press. L'introduzione offre una breve guida a ciò che insegna la storia degli studi di internet e un sommario degli ambiti chiave più importanti.

11 Si veda, per esempio, Miller, D., Slater, D. 2000. The Internet: An Ethnographic Approach. Oxford: Berg.

12 Miller, D., Slater, D. 2000. The Internet: An Ethnographic Approach. Oxford: Berg.

13 http://www.danah.org/researchBibs/sns.php

14 Non abbiamo citato fonti per queste figure solo perché non sappiamo se c'è una qualche fonte autorevole che possa essere affidabile. Invece abbiamo preferito "browsizzare" internet alla ricerca di quelle che si possono definire figure "tipiche" di base su diversi siti. Le nostre immagini per questo riflettono un internet visivo "generico" per come si presentava nei mesi di maggio e giugno del 2015.

15 C'è un riferimento bibliografico al sito cinese di Renren: Qiu, L., Lin, H., Leung, A. K.-y. 2013. «Cultural Differences and Switching of In-Group Sharing Behavior Between an American (Facebook) and a Chinese (Renren) Social Networking Site», Journal of Cross-Cultural Psychology, 44(1): 106-121.

16 Non siamo a conoscenza di una bibliografia di riferimento comparabile in lingua cinese.

17 boyd, d., Ellison, N.B. 2007. «Social Network Sites: Definition, History and Scholarship», Journal of Computer-Mediated Communication 13(1): 210-230

18 Hjorth, L. 2009. "Gifts of Presence: A Case Study of a South Korean Virtual Community, Cyworld's Mini-Hompy», Internationalising the Internet. Goggin, G., McLelland, M., eds. 237-251. London: Routledge. Hjorth, L. 2010. «The Game of Being Social: Web 2.0, Social Media, and Online Games», Iowa Journal of Communication 42(1): 73-92.

19 Chambers, D. 2013. Social Media and Personal Relationships: Online Intimacies and Networked Friendship. Basingstoke: Palgrave Macmillan. Mehdizadeh, S. 2010. «SelfPresentation 2.0: Narcissism and Self-Esteem on Facebook», Cyberpsychology, Behaviour and Social Networking, 13(4): 357-364. Rainie, L., Wellman, B. 2012. Networked. Cambridge, MA: The MIT Press.

20 Per esempio, Fuchs, C. 2013. Social Media: A Critical Introduction. London: Sage

21 Come possono gli antropologi, per esempio Gershon, I. 2011. «Un-Friend My Heart: Facebook, Promiscuity and Heartbreak in a Neoliberal Age», Anthropological Quarterly 84(4): 865-894.

22 De Tocqueville, A. 1994 (1840). Democracy in America. London: David Campbell.

23 Xiong, C., Lv, Y. 2013. "Social Network Service and Social Development in China», Studies in Communication Sciences, 13(2): 133-138.

24 A novembre del 2014 Renren aveva 45 milioni di utenti attivi ogni mese secondo http:// 
www.chinainternetwatch.com/10928/renren-q3-2014/, e nel 2015 WeChat ne aveva 600 milioni e QQ 843 milioni secondo http://www.chinainternetwatch.com/14304/ wechat-maus-reached-600-million-in-q2-2015/.

25 In questo libro una app su uno smartphone può essere una piattaforma di social media che non occorre presentare come un sito.

26 Ci sono molti libri sullo sviluppo della rete, il web e la Silicon Valley, per esempio Naughton, J. 2012. From Guttenberg to Zuckerberg. London: Quercus.

27 Come indicato in precedenza queste si basano su "tipiche" immagini online tratte da diversi siti nel periodo maggio/giugno 2015.

28 Un libro come Twitter and Society (Weller, K. et al., eds. 2013. New York: Peter Lang) mostra mediante la varietà dei suoi capitoli la tensione fra il desiderio di studiare una piattaforma nella sua ragione d'essere e il riconoscimento sia che tale piattaforma si è diversificata ormai in molti usi differenti e relative conseguenze sia che è sempre di più il prodotto dei suoi utenti.

29 Miller, D. 2011. Tales from Facebook. Cambridge: Polity Press.

30 Per uno dei pochi tentativi di osservare in modo sistematico questi processi e l'impatto dei media si veda Rantanen, T. 2005. The Media and Globalization. New York: Sage.

31 Questa rassegna certamente è carente nel senso che tiene conto solo delle pubblicazioni in inglese, pur riconoscendo che esistono molti studi locali significativi nella lingua propria del luogo considerato.

32 Non siamo qualificati per valutare tale lavoro.

33 Per esempio, Ellison, N.B., Steinfield, C., Lampe, C. 2007. "The Benefits of Facebook "Friends": Exploring the Relationship between College Students' Use of Online Social Networks and Social Capital», Journal of Computer-Mediated Communication, 12(3), 1143-1168.

34 boyd, d., Ellison, N.B. 2007. «Social Network Sites: Definition, History and Scholarship», Journal of Computer-Mediated Communication 13(1): 211.

35 boyd, d. 2008. «Facebook's Privacy Trainwreck: Exposure, Invasion, and Social Convergence», Convergence 14(1): 13-20. Livingstone, S. 2008. "Taking Risky Opportunities in Youthful Content Creation: Teenagers' Use of Social Networking Sites for Intimacy, Privacy and Self-expression», New Media \& Society 10(3): 393-411.

36 boyd, d., Ellison, N.B. 2007. «Social Network Sites: Definition, History and Scholarship», Journal of Computer-Mediated Communication 13(1): 211.

37 Una guida a molti di questi si può trovare in Wilson, R., Gosling, S., Graham, L. 2012. 'A Review of Facebook Research in the Social Sciences.' Perspectives on Psychological Science 7 (3): 203-220.

38 Baym, N. 2010. Personal Connections in the Digital Age. Cambridge: Polity Press.

39 Chambers, D. 2013. Social Media and Personal Relationships: Online Intimacies and Networked Friendship. Basingstoke: Palgrave Macmillan.

40 Noor Al-Deen, H., Hendricks, J., eds. 2012. Social Media: Usage and Impact. Lanham, MD: Lexington Books.

41 PerunesempiorecentesivedaLenhart,A.2015. «Teens,SocialMedia\&TechnologyOverview» http://www.pewinternet.org/2015/04/09/teens-social-media-technology-2015/

42 Per esempio Adorno, T.W., Horkheimer, M. 2002. Dialectic of Enlightenment. Stanford, 
CA: Stanford University Press.

43 Fuchs, C. 2013. Social Media: A Critical Introduction. London: Sage.

44 Per esempio Hunsiger, J., Senft, T., eds. 2014. The Social Media Handbook. New York: Routledge.

45 Per esempio Hanna, R., Rohm, A., Crittenden, V. L. 2010. «We're All Connected: The Power of the Social Media Ecosystem», Business Horizons, 54(3): 265-273. Jussila, J., Kärkkäinen, H., Leino, M. 2011. «Benefits of Social Media in Business-to-Business Customer Interface in Innovation", Proceedings of the 15th International Academic MindTrek Conference: Envisioning Future Media Environments: 167-174. Mangold, W.G., Faulds, D.J. 2009. «Social Media: The New Hybrid Element of the Promotion Mix», Business Horizons 52(4): 357-365. Culnan, M.J., McHugh, P.J., Zubillaga, J.I. 2010. «How Large US Companies Can Use Twitter and Other Social Media to Gain Business Value», MIS Quarterly Executive 9(4): 243-259.

46 Lomborg, S. 2014. Social Media, Social Genres: Making Sense of the Ordinary. New York: Routledge. Si vedano anche Jones, G., Schieflin, B., Smith, R. 2011. "When Friends Who Talk Together Stalk Together: Online Gossip as Meta Communication», in Thurlow, C., Mroczek, K., eds. Digital Discourse: Language in the New Media. Oxford: Oxford University Press: 26-47.

47 McLaughlin, C., Vitak, J. 2012. "Norm Evolution and Violation on Facebook», New Media \& Society 14(2): 299-315.

48 Le curatele spesso offrono la giustapposizione di casi da differenti parti del mondo, per esempio Alev Degim, I., Johnson, J., Fu, T., eds. 2015. Interpersonal Interactions Across Borders. Amsterdam: Institute of Network Cultures.

49 boyd, d. 2014. It's Complicated: The Social Lives of Networked Teens. New Haven; London: Yale University Press: 6-14. Clark, L.S. 2013. The Parent App. Oxford: Oxford University Press. Ito, M. et al. 2010. Hanging Out, Messing Around, and Geeking Out. Cambridge, MA: The MIT Press. Livingstone, S. 2009. Children and the Internet. Cambridge: Polity Press. Livingstone, S., Sefton-Green, J. 2016. The Class: Connections and Disconnections in the Digital Age. New York: New York University Press.

50 Levine, G., ed. 1971. Georg Simmel on Individuality and Social Forms. Chicago: Chicago University Press.

51 Castells, M. 1996. The Rise of the Network Society, The Information Age: Economy, Society and Culture, Vol. I. Oxford: Blackwell. Castells, M. 2000. The Information Age: Economy, Society and Culture, edizione aggiornata, vol. I. Oxford: Blackwell.

52 Castells, M. 1996. The Rise of the Network Society. The Information Age: Economy, Society and Culture, Vol. I. Oxford: Blackwell: 370.

53 Miller, D., Slater, D. 2000. The Internet: An Ethnographic Approach. Oxford: Berg. Askew, K., Wilk, R. 2002. Anthropology of Media: A Reader. Oxford: Blackwell. Postill, J. 2008. "Localizing the Internet Beyond Communities and Networks», New Media \& Society 10(3): 413-431.

54 Rainie, L., Wellman, B. 2012. Networked. Cambridge, MA: The MIT Press.

55 Si veda il Capitolo 12.

56 Si veda il Capitolo 7.

57 Boase, J., Wellman, B. 2006. "Personal Relationships: On and Off the Internet», in Perlman, D., Vangelisti, A., eds. Handbook of Personal Relations. Cambridge: Cambridge 
University Press.

58 Godelier, M. 2012. The Metamorphosis of Kinship. London: Verso.

59 Mauss, M. 1966. The Gift. London: Cohen and West.

60 Un focus sulle normative è naturalmente riconosciuto da altre discipline, per esempio i media studies. Couldry, N. 2015. "Social Media: Human Life», Social Media + Society $1(2)$.

61 Bourdieu, P. 1972. Outline to a Theory of Practice. Cambridge: Cambridge University Press.

62 Askew, K., Wilk, R. 2002. Anthropology of Media: A Reader. Oxford: Blackwell. Ginsburg, F., Abu-Lughod, L., Larkin, B., eds. 2002. Media Worlds. Berkeley, CA: University of California Press.

63 Per esempio http://www.media-anthropology.net/.

64 Coleman, G. 2010. "Ethnographic Approaches to Digital Media», Annual Review of Anthropology 39: 487-505. Horst, H., Miller, D., eds. Digital Anthropology. Oxford: Berg.

65 Landzelius, K., ed. 2006. Native on the Net. London: Routledge.

66 Bernal, V. 2014. Nation as Network. Chicago: University of Chicago Press.

67 Greschke, H. 2012. Is There a Home in Cyberspace?: The Internet in Migrants' Everyday Life and the Emergence of Global Communities. London: Routledge.

68 Oosterbaan, M. 2010. "Virtual Migration. Brazilian Diasporic Media and the Reconfigurations of Place and Space", Revue Européenne des Migrations Internationales 26(1): 81-102. Oosterbaan, M. 2010. «Virtual Re-evangelization: Brazilian Churches, Media and the Postsecular City», in Beaumont, J., Molendijk, A., Jedan, C., eds., Exploring the Postsecular: The Religious, The Political, the Urban, Leiden: Brill, 281308. Schrooten, M. 2012. "Moving ethnography online: Researching Brazilian migrants' online togetherness», Ethnic and Racial Studies 35(10): 1794-1809. McKay, D. 2011. "On the Face of Facebook: Historical Images and Personhood in Filipino Social Networking», History and Anthropology 21(4): 483-502. Nur Muhammad, R., Horst, H.A., Papoutsaki, E., Dodson, G. 2015. «Uyghur Transnational Identity on Facebook: On the Development of a Young Diaspora» Identities 23(4): 485-499.

69 Postill, J. 2008. "Localizing the Internet Beyond Communities and Networks", New Media \& Society 10(3): 413-431.

70 Coleman, G. 2012. Coding Freedom: The Ethics and Aesthetics of Hacking. Princeton, NJ: Princeton University Press.

71 Uimonen, P. 2012. Digital Drama. New York: Routledge.

72 Rappresenta un'eccezione Barendregt, B. 2012. "Diverse Digital Worlds", in Horst, H., Miller, D., eds. Digital Anthropology. Oxford: Berg, 203-224.

73 Boellstor, T. 2008. Coming of Age in Second Life. Princeton, NJ: Princeton University Press. Si veda anche Hine, C. 2000. Virtual Ethnography. London: Sage.

74 Information and Communication Technologies for Development.

75 Per esempio, Tacchi, J. 2012. «Digital Engagement», in Horst, H., Miller, D., eds. Digital Anthropology. London: Berg, 225-241.

76 Per esempio, Van Dijk, J.A.G.M. 2013. «Inequalities in the Network Society», in OrtonJohnson, K., Prior, N., eds. Digital Sociology. London: Palgrave Macmillan. 
77 Per esempio, Graham, M. 2014. «Internet Geographies: Data Shadows and Digital Divisions of Labour», in Graham, M., Dutton, W., eds. The Internet and Society. Oxford: Oxford University Press, 99-116.

78 Dalsgaard, S. 2008. «Facework on Facebook: The presentation of Self in Virtual Life and its Role in the US Elections", Anthropology Today 24(6): 8-12. Kuntsman, A., Stein, R. 2015. Digital Militarism: Israel's Occupation in the Social Media Age. Stanford, CA: Stanford University Press.

79 Broadbent, S. 2011. L'Intimité au Travail. Paris: Fyp Editions. Gershon, I. 2010. Breakup 2.0: Disconnecting Over New Media. Cornell: Cornell University Press.

80 Per esempio, Rangaswamy, N., Arora, P. 2015. «The Mobile Internet in the Wild and Every Day: Digital Leisure in the Slums of Urban India», International Journal of Cultural Studies. Kumar, N. 2014. «Facebook for Self-empowerment? A Study of Facebook Adoption in Urban India», New Media \& Society 16(7): 1122-1137.

81 Per esempio, Gingrich, A., Fox, R. G., eds. 2002. Anthropology, By Comparison. London: Routledge. Holy, L., ed. 1987. Comparative Anthropology. Oxford and New York: Blackwell.

82 Il relativismo culturale è la pratica di valutare il comportamento umano nei termini del contesto culturale locale.

\section{Capitolo 3}

1 Costa, E. 2016. Social Media in Southeast Turkey, London: UCL Press.

2 Soprattutto in ambito commerciale.

3 Miller ha vissuto vicino al suo terreno di ricerca, ma vi era presente per la maggior parte del tempo.

4 Miller, D. 1996. A Theory of Shopping. Cambridge: Polity Press.

5 Insieme a Ciara Green. Uno strano uomo che bussa alla porta da solo in Inghilterra potrebbe rivelarsi una tecnica di ricerca molto inefficace.

6 Con l'eccezione di Miller, D., Sinanan, J. 2017. Visualing Facebook. London: UCL Press.

7 Per esempio Kala Shreen del Center for Creativity, Heritage and Development, Chennai, per i nostri film relativi all'India.

8 www.ucl.ac.uk/why-we-post.

9 Boellstorff, T., Nardi, B., Pearce, C., Taylor, T. 2012. Ethnography and Virtual Worlds: A Handbook of Method. Princeton, NJ: Princeton University Press.

10 Con indiretto si intende un post che sembra essere diretto a un individuo senza specificare chi sia.

\section{Capitolo 4}

1 Siamo tuttavia consapevoli che si tratta di un campione piccolo. Come emerge dalle conclusioni di questo capitolo, pensiamo che questi numeri siano più importanti per difender- 
si dal tipo di generalizzazioni che troviamo in letteratura (tutte le persone fanno questo o quello nei social media) piuttosto che come base per una generalizzazione a tutti gli effetti.

2 Laddove uno specifico sito non sia incluso nella carta o nella tavola, questo è dovuto al fatto che si aveva un numero troppo basso di risposte a una data domanda. Ci sono casi in cui le percentuali all'interno del sito potrebbero collocarsi al $99 \%$ o al $101 \%$ invece che al $100 \%$. Ciò è dovuto all'arrotondamento dei punti decimali al numero intero più vicino.

3 McDonald, T. 2016. Social Media in Rural China. London: UCL Press.

4 Dati che derivano da una inchiesta indipendente di Wang su 238 migranti rurali nella Cina industriale.

5 Il blog di Spyer su questo è: http:// blogs.ucl.ac.uk/ global- social- media/ 2014/ 08/ 31/ thequalitative-insights- we- get- from- applying- questionnaires/.

6 Miller, D. 2011. Tales from Facebook. Cambridge: Polity Press.

7 Un'inchiesta indipendente condotta da Xinyuan Wang tra 200 utenti di smartphone nel sito della Cina industriale.

8 Maggiori particolari sull'impegno delle persone in relazione al commercio si trovano nel Capitolo 6, specificamente dedicato all'argomento.

9 McDonald, T. 2016. Social Media in Rural China. London: UCL Press. Wang, X. 2016. Social Media in Industrial China. London: UCL Press.

10 Miller, D. 2011. Tales from Facebook. Cambridge: Polity Press.

11 Bernard, H.R. 2011. Research methods in anthropology: Qualitative and quantitative approaches. Lanham, MD: Altamira Press.

12 Per ragioni etiche le risposte a tutte le nostre inchieste sono state rese anonime.

13 I libri della serie Why We Post, come elencati alla fine di questo volume.

14 Anche se sarà evidente sin da ora il fatto che spesso ci siamo sbagliati.

\section{Capitolo 5}

1 Buckingham, D. 2003. Media Education: Literacy, Learning and Contemporary Culture. Cambridge: Polity Press.

2 Ito, M., Horst, H., Bittanti, M., boyd, d., Herr-Stephenson, B., Lange, P. G., Pascoe, C. J., Robinson, L. 2008. Living and Learning with New Media: Summary of Findings from the Digital Youth Project. Cambridge, MA: The MIT Press. Ito, M. et al. 2010. Hanging Out, Messing Around, and Geeking Out. Cambridge, MA: The MIT Press.

3 Gli antropologi hanno spesso lanciato questa sfida tra apprendimento formale e informale. Si veda, per esempio: Borofsky, R. 1987. Making History: Pukapukan and Anthropological Constructions of Knowledge. Cambridge: Cambridge University Press. Akinnaso, F.N. 1992. «Schooling, Language and Knowledge in Literate and Nonliterate societies", Comparative Studies in Society and History 34: 68-109.

4 Lange, P. 2014. Kids on YouTube. Walnut Creek, CA: Left Coast Press.

5 Wesch. M. 2008. 'An Anthropological Introduction to YouTube.' YouTube, 26 July 2008. http:// www.youtube.com/ watch?v=TPAO- IZ4_ hU, (accesso 14 settembre 2014). 
6 Scobie, W. 2011. «An Anthropological Introduction to YouTube by Michael Wesch», American Anthropologist 113(4): 661-662.

7 Un contributo chiave all'antropologia dell'apprendimento viene dai lavori di Jean Lave. Si veda: Lave, J. 1993. Situated Learning: Legitimate Peripheral Participation. Cambridge: Cambridge University Press. Particolarmente influente a proposito dell'apprendimento informale online è Wenger, E. 1998. Communities of Practice: Learning, Meaning, and Identity. Cambridge: Cambridge University Press. Si veda anche, per esempio, Kimble, C., Hildreth, P.M., Bourdon, I., eds. 2008. Communities of Practice: Creating Learning Environments for Educators. Charlotte, NC: Information Age.

8 Ito, M., Horst, H., Bittanti, M., boyd, d., Herr-Stephenson, B., Lange, P. G., Pascoe, C. J., Robinson, L. 2008. Living and Learning with New Media: Summary of Findings from the Digital Youth Project. Cambridge, MA: The MIT Press.

9 boyd, d. 2014. It's Complicated: The Social Lives of Networked Teens. New Haven, CT; London: Yale University Press.

10 Clark, L.S. 2013. The Parent App. Oxford: Oxford University Press.

11 Livingstone, S., Sefton-Green, J. 2016. The Class: Connections and Disconnections in the Digital Age. New York: New York University Press.

12 Si vedano, per esempio: Cross, M. 2011. Bloggerati, Twitterati: How Blogs and Twitter are Transforming Popular Culture. Santa Barbara, CA: Praeger. Wallis, C. 2013. Technomobility in China: Young Migrant Women and Mobile Phones. New York; London: New York University Press.

13 Livingstone, S., Mascheroni, G., Ólafsson, K., Haddon, L., with EU Kids Online and Net Children Go Mobile. 2014. «Children's Online Risks and Opportunities: Comparative Findings from EU Kids Online and Net Children Go Mobile», novembre 2014. http:// eprints.Ise.ac.uk/ 60513/. Hasebrink, U., Livingstone, S., Haddon, L. 2008. Comparing Children's Online Opportunities and Risks across Europe: Cross-National Comparisons for EU Kids Online. 2nd edition. http:// eprints.lse.ac.uk/ 24368/.

14 È importante sottolineare che questa 'regola generale' di comparazione dello sviluppo economico riguarda i nostri siti di ricerca e non i paesi in cui essa si è svolta.

15 Si vedano, per esempio: Fong, V.L. 2004. Only Hope: Coming of Age under China's One-child Policy. Stanford, CA: Stanford University Press. Kipnis, A. B. 2011. Governing Educational Desire: Culture, Politics, and Schooling in China. Chicago, IL: University of Chicago Press.

16 Di default, gli utenti di Snapchat non sono capaci di inoltrare questo tipo di immagini. Tuttavia, molti utenti sanno che possono prendere facilmente le immagini di Snapchat ricevute e poi inoltrarle attraverso la funzione del cellulare 'built-in screen capture'.

17 Smith, P. K., Brain, P. 2000. «Bullying in Schools: Lessons from Two Decades of Research", Aggressive Behavior 26(1): 1-9.

18 Hull, G., Schultz, K. 2002. School's Out: Bridging Out-of-school Literacies with Classroom Practice. New York, NY: Teachers College Press.

19 Street, B. 2003. «What's "New” in New Literacy Studies? Critical Approaches to Literacy in Theory and Practice», Current Issues in Comparative Education 5(2): 77-91.

20 Potter, J. 2011. «New Literacies, New Practices and Learner Research: Across the SemiPermeable Membrane between Home and School», Lifelong Learning in Europe (3): 174-180. 
21 Filmati che illustrano questo esempio si possono trovare su: www.ucl.ac.uk/ why-we-post.

22 Si tratta di gruppi formati dalla scuola per incoraggiare e organizzare la partecipazione dei genitori nella gestione della scuola stessa.

\section{Capitolo 6}

1 Adorno, T.W. 1991. "Free time», in Bernstein, J.M., ed. The Culture Industry: Selected Essays on Mass Culture. London: Routledge, 162-170. Broadbent, S. 2011. L'Intimité au Travail. Paris: Fyp Editions. Grint, K. 2005. The Sociology of Work: Introduction. Cambridge: Polity Press.

2 Broadbent, S. 2012. «Approaches to Personal Communication», in Horst, H., Miller, D., eds. Digital Anthropology: 127-45. London, Oxford: Berg.

3 Baba, M.L. 1998. "The Anthropology of Work in the Fortune 1000: A Critical Retrospective», Anthropology of Work Review 18(4): 17-28. Jordan, A. 2003. Business Anthropology. Long Grove: Waveland Press. Ortiz, S. 1994. "Work, the Division of Labour and Co-operation», in Ingold, T., ed. Companion Encyclopedia of Anthropology. London: Taylor \& Francis. Wallman, S., ed. 1979. Social Anthropology of Work. Vol. 19. London: Academic Press.

4 Holmström, M. 1976. South Indian Factory Workers: Their Life and Their World. Cambridge: Cambridge University Press. Vidyarthi, L.P., ed. 1984. Applied Anthropology in India: Principles, Problems, and Case Studies. New Delhi: Kitab Mahal.

5 De Neve, G. 2005. The everyday Politics of Labour: Working Lives in India's Informal Economy. Oxford: Berghahn Books. Holmström, M. 1976. South Indian Factory Workers: Their Life and Their World. Cambridge: Cambridge University Press.

6 Vedi Figura 4.21.

7 https://press.linkedin.com/about-linkedin (accesso 28 agosto 2015).

8 L'espressione italiana per 'artisanal business', imprese artigiane, comprende molto di più che artigianato, e include ogni tipo di attività eseguita a mano, dall'agricoltura all'idraulica, dalla ceramica all'acconciatura.

9 boyd, d. 2014. It's Complicated: The Social Lives of Networked Teens. New Haven, CT; London: Yale University Press.

10 Fuller, C. J., Narasimhan, H. 2007. «Information Technology Professionals and the Newrich Middle Class in Chennai (Madras)», Modern Asian Studies 41(1): 121-150. Nisbett, N. 2009. Growing Up in the Knowledge Society: Living the IT Dream in Bangalore. New Delhi: Routledge.

11 http://blogs.ucl.ac.uk/global-social-media/2013/11/24/what-will-we-learn-fromthe-fall-of-facebook/

12 È solo una supposizione, ma forse il motivo di questa attenzione limitata è una sorta di consapevolezza che se un'azienda dovesse davvero offenderli ci sarebbero sempre più alternative disponibili.

13 Un'espansione del codice a barre che proliferò in molti settori (persino quello delle pietre tombali) per un po' di tempo: http://www.theatlantic.com/technology/archive/2014/05/ 
qr-codes-for-the- dead/370901/.

14 Lee, K., Kim, J. H., Woo, W. T., eds. 2009. Power and Sustainability of the Chinese State. New York: Routledge.

15 http://www.nytimes.com/2008/07/26/business/worldbusiness/26internet.html?_r=0.

16 Che è anche il perché la nostra inchiesta li mostra come pubblicità "liking", anche se non acquistano poi il prodotto. Per ulteriori dettagli si veda la discussione della Fig. 4.19 riportata sopra.

17 Goldfarb, A., Tucker, C. 2011. «Online Display Advertising: Targeting and Obtrusiveness", Marketing Science 30(3): 389-404.

18 Questo non è così evidente dalla Fig. 4.19, ma la domanda posta era leggermente diversa. In ogni caso abbiamo inteso le prove etnografiche raccolte per 15 mesi come di gran lunga più autorevoli delle risposte a una survey superficiale.

19 È il soggetto di uno dei nostri film su: ucl.ac.uk/why-we-post.

20 Soggetto di un altro film su: ucl.ac.uk/why-we-post.

21 Lo stiamo osservando dalla prospettiva degli utenti quotidiani. Riconosciamo che per un'azienda che paga molto poco la pubblicità sui social media, anche un utilizzo proporzionalmente debole può avere un valore.

22 Si veda anche Chu, J.Y. 2010. Cosmologies of Credit: Transnational Mobility and the Politics of Destination in China. Durham, NC: Duke University Press.

23 Staord, C. 1995. The Roads of Chinese Childhood: Learning and Identification in Angang. Cambridge; New York: Cambridge University Press.

24 http://www. wantchinatimes.com/news-subclass-cnt.aspx?id=20140205000127 \&cid $=1102$.

25 Zelizer, V. 2011. Economic Lives. Princeton, NJ: Princeton University Press.

26 McKay, D. 2007. «Sending Dollars Shows Feeling: Emotions and Economies in Filipino Migration", Mobilities 2(2): 175-194.

27 Queste non dovrebbero quindi essere confuse con altre nuove pratiche digitali di commercio come Airbnb o Uber, che in tutto e per tutto stanno usando le stesse funzioni digitali per guadagnare denaro.

28 Sahlins, M. 1972. Stone Age Economics. Chicago: Aldine-Atherton.

\section{Capitolo 7}

1 Si veda Prensky, M. 2001. "Digital Natives, Digital Immigrants», On the Horizon 9(5): 1-6.

2 Si veda Miller, D., Slater, D. 2000. The Internet: An Ethnographic Approach. Oxford: Berg.

3 Si veda il dibattito più articolato sul timore della società verso le nuove tecnologie in Marvin, C. 1988. When Old Technologies were New. New York: Oxford University Press.

4 Platone 2008 (360 BC). Phaedrus. Charleston, SC: Forgotten Books.

5 Si veda Turkle, S. 2011. Alone Together: Why We Expect More from Technology and Less from Each Other. New York: Basic Books. 
6 Si veda Carr, N. 2011. The Shallows: What the Internet Is Doing to Our Brains. New York: W.W. Norton \& Company.

7 Horst, H., Miller, D., eds. 2012. Digital Anthropology. London: Berg, 11-15. Vedi anche, Butler, J. 1990. Gender Trouble. New York: Routledge. Baker, L.D. 1998. From Savage to Negro: Anthropology and the Construction of Race. Los Angeles, CA: University of California Press.

8 Miller, D., Sinanan, J. 2014. Webcam. Cambridge: Polity Press.

9 Si veda la discussione dei concetti chiave usati per analizzare modalità differenti della connessione personale in Baym, N. 2010. Personal Connections in the Digital Age. Cambridge: Polity Press, 6-12.

10 Si veda Goffman, E. 1975. Frame Analysis. Harmondsworth: Penguin.

11 Si veda Aarsand, P.A. 2008. «Frame Switches and Identity Performances: Alternating Between Online and Online», Text \& Talk. 28(2): 147-165.

12 Lomborg, S. 2014. Social Media, Social Genres: Making Sense of the Ordinary. London: Routledge.

$13 \mathrm{Su}$ Facebook è possibile, ma tale funzione non è molto usata dai nostri informatori.

14 Si veda Yan, Y. 1996. The Flow of Gifts: Reciprocity and Social Networks in a Chinese Village. Stanford, CA: Stanford University Press.

15 In Cina si può anche usare la funzione gruppo di QQ ('QQ qun'), simile a quella di WhatsApp, che consiste nell'usare i contatti offline per creare gruppi di conversazione online. Come con WhatsApp, ciò permette di avere una varietà di gruppi definiti - più in linea con il modo in cui ci relazioniamo agli amici offline.

16 Si veda la discussione della "socialità modulabile" nel Capitolo 1 di questo volume.

17 Si veda Jankowiak, W. 2002. "Proper Men and Proper Women: Parental Affection in the Chinese Family», in Brownell, S., Wasserstrom, J., eds. Chinese Femininities/Chinese Masculinities: A Reader. Berkeley, CA: University of California Press, 361-381.

18 Miller, D. 2015. "The Tragic Dénouement of English Sociality», Cultural Anthropology 30(2): 336-357. È anche l'argomento principale in Miller, D. 2016. Social Media in an English Village. London: UCL Press.

19 Si veda Broadbent, S. 2012. «Approaches to Digital Communication», in Horst, H., Miller, D., eds. Digital Anthropology. London: Berg, 127-145.

20 Broadbent, S. 2012. «Approaches to Digital Communication» in Horst, H., Miller, D., eds. Digital Anthropology. London: Berg, 127-145.

21 Vedi la Figura 4.2.

22 Queste generalizzazioni variano in base alla variabilità del tipo di uso delle piattaforme. Le persone non si preoccupano tanto dei follower su Twitter o su Instagram, che non si considerano come delle vere e proprie connessioni sociali. Si preoccupano, invece, quando accade qualcosa su piattaforme in cui c'è la connotazione personale delle relazioni.

23 Argomento centrale in McDonald, T. 2016. Social Media in Rural China. London: UCL Press.

24 Il modo in cui i social media hanno agevolato la trasformazione delle reti sociali delle persone e la creazione di forme di socialità individuali è un argomento chiave in Costa, E. 2016. Social Media in Southeast Turkey. London: UCL Press.

25 Si veda Sennett, R. 1977. The Fall of Public Man. New York: Knopf. 
26 Si veda Giddens, A. 1991. Modernity and Self-identity: Self and Society in the Late Modern Age. Cambridge: Polity Press.

27 Goffman, E. 1975. Frame Analysis. Harmondsworth: Penguin. Si veda anche Brubaker, R., Cooper, F., 2000. "Beyond "Identity"», Theory and Society, 29: 1-47.

28 Si veda il Capitolo 11.

29 Si veda Miller, D. 2011. Tales from Facebook. Cambridge: Polity Press.

30 Si veda l'analisi dettagliata in Wang, X. 2016. Social Media in Industrial China. London: UCL Press.

31 Si veda Bargh, J.A. et al. 2002. "Can You See the Real Me? Activation and Expression of the "True Self" on the Internet", Journal of Social Issues 58(1): 33-48.

32 Per una critica complessiva di quest' idea che le tecnologie digitali riducono la nostra umanità si veda Miller, D., Sinanan, J. 2014. Webcam. Cambridge: Polity Press, Capitolo1.

33 Turkle, S. 1997. Life on the Screen: Identity in the Age of the Internet. New York: Simon and Schuster.

34 Si veda Baym, N. 1999. Tune In, Log On: Soaps, Fandom, and Online Community. New York: Sage.

35 Si veda Hampton, K., Wellman, B. 2003. «Neighboring in Netville: How the Internet Supports Community and Social Capital in a Wired Suburb", City and Community 2(4): 277-311.

36 Si veda Livingstone, S. 2008. "Taking Risky Opportunities in Youthful Content Creation: Teenagers' Use of Social Networking Sites for Intimacy, Privacy and Selfexpression", New Media \& Society 10: 393-411.

\section{Capitolo 8}

1 Rheingold, H. 1993. Virtual Community: Homesteading in the Electronic Frontier. New York: Adddison-Wesley. Turkle, S. 1997. Life on the Screen: Identity in the Age of the Internet. New York: Simon and Schuster.

2 Plant, S. 1997. Zeros and Ones: Digital Women and the New Technoculture. London: Fourth Estate.

3 Fra gli altri, si veda Haraway, D. 1991. "A Cyborg Manifesto: Science, Technology and Social Feminism in the Late Twentieth Century», in Simians, Cyborgs and Women: The reinvention of Nature. New York: Routledge.

4 Ortner, S.B. 1972. "Is Female to Male as Nature Is to Culture?», Feminist Studies 1(2): 5-31.

5 Castells, M. 1997. The Power of Identity. The Information Age: Economy, Society and Culture Vol II. Cambridge, MA; Oxford: Blackwell. Shade, L. R. 2002. Gender and Community in the Social Construction of the Internet. New York: Peter Lang.

6 Wajcman, J. 2004. TechnoFeminism. Cambridge: Polity Press.

7 Livingstone, S. 2008. "Taking Risky Opportunities in Youthful Content Creation: Teenagers' Use of Social Networking Sites for Intimacy, Privacy and Self-expression", New Media \& Society 10: 393-411. Van Doorn, N., Van Zoonen, L., Wyatt, S. 2007. 
"Writing from Experience: Presentations of Gender Identity on Weblogs», European Journal of Women's Studies 14(2): 143-159. Paechter, C. 2013. «Young Women Online: Collaboratively Constructing Identities», Pedagogy, Culture and Society 21(1): 111127. Gray, M.L. 2009. Out in the Country: Youth, Media, and Queer Visibility in Rural America. New York: New York University Press.

8 boyd, d. 2014. It's Complicated: The Social Lives of Networked Teens. New Haven, CT; London: Yale University Press.

9 Con l'espressione social media pubblici ('public-facing') intendiamo quegli spazi online visibili a un grande numero di persone, da centinaia fino a un numero illimitato.

10 Butler, J. 1990. Gender Trouble. New York: Routledge.

11 Si veda Figura 4.7.

12 Van Dijck, J. 2013. "You Have One Identity": Performing the Self on Facebook and LinkedIn", Media, Culture \& Society 35(2): 199-215.

13 Miller, D., Sinanan, J. 2017. Visualising Facebook. London: UCL Press.

14 Lesbian Gay Bisexual Transgender. Per approfondimenti sulla questione della visibilità, si veda, per esempio, Gray, M.L. 2009. Out in the Country: Youth, Media, and Queer Visibility in Rural America. New York: New York University Press.

\section{Capitolo 9}

1 Riguardo all'impatto dei telefoni cellulari, si veda per esempio Jeffrey, R., Doron, A. 2013. The Great Indian Phone Book. London: Hurst. Wallis, C. 2013. Technomobility in China: Young Migrant Women and Mobile Phones. New York; London: New York University Press. Miller, D., Horst, H. 2006. The Cell Phone. Oxford: Berg.

2 Il brand Samsung ha avuto il primato nel mercato globale dal 2012 fino a oggi per gli smartphone a basso costo. Si veda http://www.idc.com/prodserv/smartphone-market-share.jsp (accesso il 26 agosto 2015).

3 Una figura chiave in tale dibattito è stata quella di Amartya Sen. Si veda, ad esempio, Sen, A. 1992. Inequality Re-examined. Cambridge, MA: Harvard University Press. Un testo più recente che discute le cause delle disuguaglianze contemporanee è Piketty, T. 2014. Capital in the Twenty-First Century. Cambridge, MA: Belknap Press.

4 Bourdieu, P. 1984. Distinction: A Social Critique of the Judgment of Taste. Cambridge, MA: Harvard University Press.

5 Skeggs, B. 1997. Formations of Class and Gender: Becoming Respectable. London: Sage.

6 Tacchi, J. 2012. «Digital Engagement», in Horst, H., Miller, D., eds. Digital Anthropology. London: Berg, 225-241.

7 Graham, M. 2014. «Internet Geographies: Data Shadows and Digital Divisions of Labour», in Graham, M., Dutton, W., eds. Society and the Internet. Oxford: Oxford University Press. 99-116.

8 C'è a questo proposito molta letteratura potenzialmente rilevante. Una che ammettiamo di aver grandemente trascurato, in quanto spostata sulla policy e non sull'etnografia, è quella sull'ITC e i suoi sviluppi. Si veda, per esempio Slater, D. 2014. New Media, 
Development and Globalization: Making Connections in the Global South. Cambridge: Polity Press. Per un approccio influenzato da Sen, si veda Kleine, D. 2013. Technologies of Choice: ICT's Development and the Capabilities Approach. Cambridge, MA: The MIT Press.

9 Chen, W., Wellman, B. 2004. «The Global Digital Divide Within and Between Countries», IT \& Society 1(7): 39-45. Zickuhr, K. 2013. Who's Not Online and Why. Rep. Washington DC: Pew Research Center's Internet \& American Life Project.

10 Warschauer, M. 2004. Technology and Social Inclusion: Rethinking the Digital Divide. Cambridge, MA: The MIT Press.

11 Rainie, L., Wellman, B. 2012. Networked. Cambridge, MA: The MIT Press, 257-259.

12 Rheingold, H. 2012. Net Smart: How to Thrive On Line. Cambridge, MA: The MIT Press.

13 Graham, M. 2014. «Internet Geographies: Data Shadows and Digital Divisions of Labour», in Graham, M., Dutton, W., eds. Society and the Internet. Oxford: Oxford University Press, 99-116.

14 La visione tecno-utopistica è riflessa negli argomenti distopici secondo cui la tecnologia sarebbe un male per la società poiché ci rende dipendenti, più isolati, più esposti al controllo istituzionale e alienati. Si veda per esempio Carr, N. 2011. The Shallows: What the Internet Is Doing to Our Brains. New York: W. W. Norton \& Company. Turkle, S. 2011. Alone Together: Why We Expect More from Technology and Less from Each Other. New York: Basic Books. Morozov, E. 2012. The Net Delusion: The Dark Side of Internet Freedom. New York, NY: Public Affairs.

15 Fra i migliori che conosciamo ci sono: Kelly, K. 1994. Out of Control: The Rise of Neobiological Civilization. Reading, MA: Addison-Wesley. Johnson, S. 2001. Emergence: The Connected Lives of Ants, Brains, Cities, and Software. New York: Scribner. Shirky, C. 2008. Here Comes Everybody: The Power of Organizing without Organizations. New York: Penguin.

16 Rheingold, H. 1993. Virtual Community: Homesteading in the Electronic Frontier. New York: Addison-Wesley. Turkle, S. 1997. Life on the Screen: Identity in the Age of the Internet. New York: Simon and Schuster.

17 Barlow, J., «A Declaration of the Independence of Cyberspace.' Electronic Frontier Foundation» 8 February 1996. https://www.eff.org/it/cyberspace-independence (accesso 7 luglio 2015).

18 boyd, d. 2014. It's Complicated: The Social Lives of Networked Teens. New Haven, CT; London: Yale University Press, 16.

19 boyd, d. 2014. It's Complicated: The Social Lives of Networked Teens. New Haven, CT; London: Yale University Press, 23.

20 boyd, d. 2013. "White Flight in Networked Publics? How Race and Class Shaped American Teen Engagement with Myspace and Facebook», in Nakamura, L. and ChowWhite, P., eds. Race after the Internet. New York: Routledge, 203-222.

21 Clark, L.S. 2013. The Parent App. Oxford: Oxford University Press.

22 Un esempio importante è Qiu, J.L. 2009. Working-Class Network Society: Communication Technology and the Information Have-Less in Urban China. Cambridge. Cambridge, MA: The MIT Press. Wallis, C. 2013. Technomobility in China: Young Migrant Women and 
Mobile Phones. New York; London: New York University Press.

23 Questo non è indice di mancanza di attenzione dei genitori per i figli; per tradizione c'è un'aspettativa che si tratti di un'esperienza temporanea e non di un'adozione informale. Sandra e tutti i suoi fratelli e sorelle furono reclamati dai loro genitori e sono ancora attaccati ai legami familiari.

24 Bahia era il centro dell'impegno coloniale portoghese nel Nuovo Mondo, basato prevalentemente sul lavoro degli schiavi nelle piantagioni di prodotti come zucchero e canna da zucchero. Secondo un censimento recente, Salvador, la capitale regionale, ha più di 700.000 persone di discendenza africana; è la popolazione nera più estesa fuori dall'Afri$\mathrm{ca}$, e anche la più vulnerabile localmente in termini socio-economici.

25 Ci sono casi in cui questa opportunità migliora la vita del/la figlio/a, dandogli/le l'opportunità di studiare ed essere trattato/a con rispetto. Comunque, la maggioranza di questi scambi lascia profonde cicatrici quando il/la figlio/a è trattato/a come un/a servo/a, spesso preso/a per occuparsi di altri ragazzi più o meno della stessa età, ma senza ricevere l'attenzione emozionale data ai veri figli e figlie.

26 Weber, M. 2002 (1905). The Protestant Ethic and the Spirit of Capitalism. London: Penguin Books.

27 Spesso ci si riferisce alla riproduzione della disuguaglianza, da parte di coloro che ne fanno esperienza, come "violenza simbolica", delineata da Bourdieu, P., Wacquant, L. 1992. Symbolic Violence. An Invitation to Reflective Sociology. Chicago, IL: University of Chicago Press, 167-173.

28 Bourdieu, P. 1984. Distinction: A Social Critique of the Judgment of Taste. Cambridge, MA: Harvard University Press.

29 Si vedano esempi di queste pratiche in Miller, D., Sinanan, J. 2017. Visualising Facebook. London: UCL Press.

30 Il termine "vlogger" è una contrazione di "video blogger". Descrive il gruppo di persone che creano contenuti online registrando e condividendo video, in particolare tramite YouTube.

31 Nella ricerca antropologica i meccanismi di livellamento sono stati descritti come parte delle società di contadini, e funzionano inibendo gli sforzi individuali di evolversi economicamente. Questo livellamento si riconduce a una concezione condivisa dei beni comuni come risorsa scarsa; si può accrescere la propria ricchezza solo a scapito degli altri. Questo modello si è evoluto a partire dallo studio delle società contadine, ma si applica a una più ampia varietà di casi. Si veda Rubel, A. J. 1977. "Limited Good" and "Social Comparison": Two Theories, One Problem», Ethos 5(2): 224-238.

\section{Capitolo 10}

1 Tra le altre fonti si vedano http://www.al-monitor.com/pulse/originals/2013/09/turkeysakp-twitter-election.html; http://www.wsj.com/articles/SB1000142412788732352700 4 579079151479634742; http://www.hurriyetdailynews.com/ruling-akp-hiresthousands-for-new-social-media-campaign.aspx?pageID=238\&nID =54479\& \& NewsCa$\mathrm{tID}=338$. 
2 Si veda Castells, M. 1996. The Rise of the Network Society. The Information Age: Economy, Society and Culture, Vol. I. Oxford: Blackwell and Castells, M. 1997. The Power of Identity. The Information Age: Economy, Society and Culture, Vol II. Cambridge, MA; Oxford: Blackwell; per approfondire alcune tra le più influenti teorie su internet $\mathrm{e}$ sull'organizzazione delle reti si vedano anche Oates, S., Owen, D., Gibson, R. K., eds. 2006. The Internet and Politics. Citizens, Voters and Activists. New York: Routledge. Klotz, R. J. 2004. The Politics of Internet Communication. Lanham, MD: Rowman \& Littlefield.

3 Si veda Fountain, J. E. 2001. Building the Virtual State: Information Technology and Institutional Change. Washington, DC: Brookings Institution Press; Narayan, G. 2007. «Addressing the Digital Divide: E-governance and M-governance in a Hub and Spoke model», The Electronic Journal on Information Systems in Developing Countries 31(1): 1-14, e Norris, P. 2001. Digital Divide: Civic Engagement, Information Poverty, and the Internet Worldwide. Cambridge: Cambridge University Press.

4 Papacharissi, Z. 2010b. "The virtual sphere 2.0: The Internet, the Public Sphere, and Beyond", in Chadwick, A., Howard, P.N., eds. Routledge Handbook of Internet Politics. Oxford: Taylor \& Francis, 230-245. Papacharissi, Z. 2004. «Democracy Online: Civility, Politeness, and the Democratic Potential of Online Political Discussion Groups», New Media \& Society 6(2): 259-283.

5 Gerbaudo, P. 2012. Tweets and the Streets: Social media and contemporary activism. London: Pluto Press. Clay. S. 2011. "The Political Power of Social Media», Foreign Affairs 90: 128-141. Seib, P. 2012. Real-time Diplomacy: Politics and Power in the Social Media Era. Basingstoke: Palgrave Macmillan.

6 Chadwick, A., Howard, P. N., eds. 2010. Routledge Handbook of Internet Politics. Oxford: Taylor \& Francis. Postill, J. 2012. «Digital Politics and Political Engagement». In Horst, H., Miller, D., eds. Digital Anthropology. London: Berg.

7 Heeks, R. 2001. «Building E-governance for Development: A Framework for National and Donor Action»: http://www.man.ac.uk/idpm/idpm_dp.htm\#ig. Chadwick, A. 2003. «Bringing E-Democracy Back In: Why it Matters for Future Research on E-Governance», Social Science Computer Review 21(4): 443-55. Dawes, S. S. 2008. «The Evolution and Continuing Challenges of E-governance», Public Administration Review 68(1): 86-102.

8 Dahlberg, L. 2001. «The Internet and Democratic Discourse: Exploring the Prospects of Online Deliberative Forums Extending the Public Sphere», Information, Communication \& Society 4(4): 615-633. Fenton, N., Barassi, V. 2011. «Alternative Media and Social Networking Sites: The Politics of Individuation and Political Participation", The Communication Review 14(3): 179-196. Habermas, J. 1969. The Structural Transformation of the Public Sphere: An Inquiry into a Category of Bourgeois Society. Cambridge, MA: The MIT Press.

9 Gerbaudo, P. 2012. Tweets and the Streets: Social Media and Contemporary Activism. London: Pluto Press. Hussain, M.M., Howard, P.N. 2012. «Opening Closed Regimes: Civil Society, Information Infrastructure, and Political Islam» in Anduiza, E., Jensen, M., Jorba, L., eds. Comparing Digital Politics: Civic Engagement and Political Participation. Cambridge: Cambridge University Press. Hussain, M.M., Howard, P.N. 2013. «What Best Explains Successful Protest Cascades? Icts and the Fuzzy Causes of the Arab Spring», 
International Studies Review 15(1): 48-66. Lim, M. 2012. "Clicks, Cabs, and Coffee Houses: Social Media and Oppositional Movements in Egypt, 2004-2011», Journal of Communication 62(2): 231-248. Salvatore, A., ed. 2011. "Between Everyday Life and Political Revolution: The Social Web in the Middle East». Oriente Moderno, n.s. XCI/1, 2011. Tufekci, Z. 2014. "The Medium and the Movement: Digital Tools, Social Movement Politics, and the End of the Free Rider Problem», Policy \& Internet 6 (2): 202-208: Tufekci, Z., Wilson, C. 2012. «Social Media and the Decision to Participate in Political Protest: Observation from Tahrir Square», Journal of Communication 62 (2): 363-379.

10 Fuchs, C. 2012. «Social Media, Riots, and Revolutions», Capital \& Class 36(3): 383391. Morozov, E. 2012. The Net Delusion: The Dark Side of Internet Freedom. New York, NY: Public Affairs.

11 Coleman, G. 2012. «Phreaks, Hackers, and Trolls: The politics of Transgression and Spectacle», in Maniberg, M., ed. The Social Media Reader. New York: New York University Press, 99-119. Coleman, G. 2014. Hacker, Hoaxer, Whistleblower, Spy: The Many Faces of Anonymous. London; Brooklyn, NY: Verso.

12 Anche se diverse analisi sul ruolo della politica in rete e sui social rispetto all'impegno politico contribuiscono ai dibattiti su democrazia, società civile e governance, esse prendono l'avvio da una posizione simile, impegnandosi nel definire che cosa, al livello locale, si intenda con impegno politico. Si veda, per esempio: Tufekci, Z. 2014. «The Medium and the Movement: Digital Tools, Social Movement Politics, and the End of the Free Rider Problem», Policy \& Internet 6(2): 202-208. Tufekci, Z., Wilson, C. 2012. «Social Media and the Decision to Participate in Political Protest: Observation from Tahrir Square», Journal of Communication 62(2): 363-379. Lim, M. 2012. "Clicks, Cabs, and Coffee Houses: Social Media and Oppositional Movements in Egypt, 2004-2011», Journal of Communication 62(2): 231-248. Lim, M. 2013. «Many Clicks but Little Sticks: Social Media Activism in Indonesia», Journal of Contemporary Asia 43(4): 636-657; Morozov, E. 2009. "Iran: Downside to the "Twitter Revolution"”, Dissent 56(4): 10-14.

13 Uno degli esempi più recenti è l'Hong Kong Umbrella Movement/Occupy Central. Si veda Fu, K.W., Chan, C.H. 2015. «Networked Collective Action in the 2014 Hong Kong Occupy Movement: Analysing a Facebook Sharing Network», International Conference on Public Policy, ICPP 2015. Tsui, L. 2015. "The Coming Colonization of Hong Kong Cyberspace: Government Responses to the Use of New Technologies by the Umbrella Movement», Chinese Journal of Communication, 8(4): 1-9.

14 Per discussioni più comprensive del modo in cui i social media si usano in rapporto alla politica, si consultino i volumi singoli di questa serie.

15 Per la censura di internet in Cina si veda MacKinnon, R. 2008. «Flatter World and Thicker Walls? Blogs, Censorship and Civic Discourse in China», Public Choice 134(12): 31-46. Per quanto riguarda la censura in Turchia, si veda Akdeniz, Y., Altiparmak, K. 2008. «Internet: Restricted Access: A critical Assessment of Internet Content Regulation and Censorship in Turkey», in Akdenizli, B., ed. 2015. Digital Transformations in Turkey: Current Perspectives in Communication Studies. Lanham, MD: Lexington Books.

16 Noelle-Neumann, E. 1974. "The Spiral of Silence: A Theory of Public Opinion», Journal of Communication 24(2): 43-51. Il Pew Research Center ha indagato il ruolo della "spira- 
le del silenzio" tra alcuni utenti di social media negli Stati Uniti, mettendo in luce come molti di essi evitino di postare punti di vista su questioni politiche nel momento in cui non sono sicuri che i rispettivi amici possano trovarsi d'accordo con loro. Hampton, K.N., Rainie, L., Lu, W., Dwyer, M., Shin, I., Purcell, K. 2014. «Social Media and the "Spiral of Silence"”, Pew Research Center, Washington, DC. http:// www.pewinternet. org/2014/08/26/social-media-and-the-spiral-of-silence/.

17 Brants, K. 2005. Guest editor's introduction: «The Internet and the Public Sphere», Political Communication 22(2): 143-6; Papacharissi, Z. 2002. «The Virtual Sphere: The Internet as a Public Sphere», New Media \& Society 4(1): 9-27; Poster, M. 1997. "Cyberdemocracy: Internet and the Public Sphere», Internet Culture 201-218.

18 King, G., Pan, J., Roberts, M.E. 2013. «How Censorship in China Allows Government Criticism But Silences Collective Expression", American Political Science Review 107(2): 326-343.

19 PKK è l'organizzazione militante curda che è stata impegnata in un conflitto armato contro lo stato turco dal 1984 fino al 2013, quando venne dichiarato il cessate il fuoco.

20 Ho, C. 2000. «Popular Culture and the Aestheticization of Politics: Hegemonic Struggle and Postcolonial Nationalism in Trinidad Carnival», Transforming Anthropology 9(1):3-18.

\section{Capitolo 11}

1 Si veda Hjorth, L., Hendry, N. 2015. «A Snapshot of Social Media: Camera Phone Practices», Social Media+Society, 1(1), 1-3. Gibbs, M., Meese, J., Arnold, M., Nansen, B., Carter, M. 2015. «\#Funeral and Instagram: Death, Social Media, and Platform Vernacular», Information, Communication \& Society 18(3): 255-268, e Cruz, E.G., Meyer, E. 2012. "Creation and Control in the Photographic Process: iPhones and the Emerging Fifth Moment of Photography», Photographies 5(2), per approfondire la relazione tra pratiche visuali e piattaforme di social media.

2 Ginsburg, F. 1995. «Mediating Culture: Indigenous Media, Ethnographic Film, and the Production of Identity", Fields of vision: Essays in Film Studies, Visual Anthropology, and Photography. Berkeley, CA: University of California Press, 258; MacDougall, D. 2005. The Corporeal Image: Film, Ethnography, and the Senses. Princeton, NJ: Princeton University Press; Sprague, S. 1978. «How I See the Yoruba See Themselves», Studies in the Anthropology of Visual Communications. 5(1): 9-29; Edwards, E. 1992. Anthropology and Photography 1860-1929. Royal Anthropological Institute; Pinney, C. 2011. Photography and Anthropology. London: Reaktion.

3 "Osservando l'ambito dei post, e delle foto in particolare, possiamo fare un'etnografia dell'ambito delle relazioni declinate culturalmente, mescolate e codificate nel visuale": Pink, S. 2001. 'Visual Ethnography.' Images, Media and Representation in Research. London; Thousand Oaks; New Delhi: Sage. Si veda anche Banks, M., Morphy, H., eds. 1997. Rethinking Visual Anthropology. New Haven, CT: Yale University Press.

4 Ginsburg, F. 1995. «Mediating Culture: Indigenous Media, Ethnographic Film, and the Production of Identity», Fields of Vision: Essays in Film Studies, Visual Anthropology, and 
Photography. Berkeley, CA: University of California Press. MacDougall, D. 2005. The Corporeal Image: Film, Ethnography, and the Senses. Princeton, NJ: Princeton University Press. Si veda inoltre Bateson, G., Mead, M. 1942. «Balinese character. A photographic analysis», Special Publications of the New York Academy of Science: 17-92. Marshall, J. A Kalahari Family. Film, 2002. Gardner, R. The Nuer, Film, 1971.

5 Miller, D., Sinanan, J. 2017. Visualising Facebook. London: UCL Press.

6 Turkle, S. 1997. Life on the Screen: Identity in the Age of the Internet. New York: Simon and Schuster.

$7 \mathrm{Si}$ veda http://www.theguardian.com/media-network/media-network-blog/2014/ mar/13/selfie-social-media-love-digital-narcassism http:/www.theguardian.com/ technology/2013/dec/06/selfies-status-updates-digital-bragging-web

8 All'opposto, si veda http://www.theguardian.com/commentisfree/2014/sep/11/ when-taking-selfies-in-trinidad-its-whats-on-the-outside-that-matters

9 Per approfondire l'uso sociale della fotografia, cfr. Van House, N.A., Davis, M. 2005. "The Social Life of Camera Phone Images", Proceedings of the Pervasive Image Capture and Sharing: New Social Practices and Implications for Technology Workshop (PICS 2005) at the Seventh International Conference on Ubiquitous Computing (UbiComp 2005). Voida, A., Mynatt, E.D. 2005. "Six Themes of the Communicative Appropriation of Photographic Images", Proceedings of the SIGCHI Conference on Human Factors in Computing Systems. 171-180. ACM. David, G. 2010. "Camera Phone Images, Videos and Live Streaming: A Contemporary Visual Trend», Visual Studies 25(1): 89-98. Okabe, D., Ito, M. 2006. «Everyday Contexts of Camera Phone Use: Steps Toward Technosocial Ethnographic Frameworks», Höflich, J. R., Hartmann, M., eds. Mobile Communication in Everyday Life: Ethnographic Views, Observations and Reflections. Berlin: Frank \& Timme, 79-102.

10 Cfr. Hjorth, L. 2007. «Snapshot of Almost Contact: the Rise of Camera Phone Practices and a Case Study in Seoul, Korea», Continuum: Journal of Media \& Cultural Studies 21(2): 227-238, e Pink, S. 2011. «Amateur Photographic Practice, Collective Representation and the Constitution of Place», Visual Studies 26(2): 92-101.

11 Vedi Dijck, J., van. 2008. «Digital Photography: Communication, Identity, Memory», Visual Communication 7: 57-76.

12 Vedi Costa, E. 2016. Social Media in Southeast Turkey. London: UCL Press, e Venkatraman, S. 2017. Social Media in South India. London: UCL Press. Capitolo 3.

13 'Aburrido' è il termine spagnolo per 'annoiato' and 'fome' lo slang cileno per 'annoiare'.

14 Bourdieu, P., Whiteside, S. 1996. Photography: A Middle-brow Art. Stanford, CA: Stanford University Press.

15 Bourdieu, P., Bourdieu, M. C. 2004. «The peasant and photography», Ethnography, 5(4): 601-616.

16 Per ulteriori approfondimenti sulla relazione fra libri a fumetti e aumento dell'alfabetizzazione si veda Bitz, M. 2004. "The Comic Book Project: The Lives of Urban Youth", Art Education 57(2): 33-46. Si veda anche Bitz, M. 2010. When Commas Meet Kryptonite: Classroom Lessons from the Comic Book Project. Language and Literacy Series. New York: Teachers College Press.

17 Si veda Spyer, J. 2017. Social Media in Emergent Brazil. London: UCL Press.

18 Ritchie, D. 2005. "Frame-Shifting in Humor and Irony», Metaphor and Symbol 20(4): 288. 
19 Kermit la Rana offre un buon esempio di come i social media agevolino la diffusione globale di immagini visive consentendo, allo stesso tempo, una rapida ricontestualizzazione locale.

20 Ito, M. 2005. «Mobile phones, Japanese Youth, and the Re-placement of Social Contact», Mobile Communication: 131-148. Ito, M., Okabe, D. 2005. «Intimate Visual Co-presence», Position paper for the Seventh International Conference on Ubiquitous Computing, Tokyo. Okabe, D. 2006. «Everyday Contexts of Camera Phone Use: Steps Toward Techno-social Ethnographic Frameworks", Mobile Communications in Everyday Life: Ethnographic Views, Observations, and Reflections. Berlin: Frank \& Timme, 79-102.

21 Per un'ampia discussione sulle fotocamere dei telefoni cellulari e gli spazi pubblici e privati, si veda Lasén, A., Gómez-Cruz, E. 2009. «Digital Photography and Picture Sharing: Redefining the Public/private Divide», Knowledge, Technology \& Policy 22(3): 205-215.

22 Lindtner, S. et al. 2011. "Towards a Framework of Publics: Re-encountering Media Sharing and its User», ACM Transactions on Computer-Human Interaction (TOCHI) 18(2): 5. Ito, M., Okabe, D. 2005. «Intimate Visual Co-presence», Position paper for the Seventh International Conference on Ubiquitous Computing, Tokyo. Boellstorff, T. 2008. Coming of Age in Second Life. Princeton, NJ: Princeton University Press.

23 Lindtner, S. et al. 2011. "Towards a Framework of Publics: Re-encountering Media Sharing and its User», ACM Transactions on Computer-Human Interaction (TOCHI) 18(2): 5. Warner, M. 2002. «Publics and Counterpublics», Public Culture 14(1): 49-90.

24 Marwick, A. 2011. «I Tweet Honestly, I Tweet Passionately: Twitter Users, Context Collapse, and the Imagined Audience», New Media \& Society 13(1): 114-133.

25 Bubel, C.M. 2008. «Film Audiences as Overhearers», Journal of Pragmatics 40: 55-71.

26 Molti dei filmati realizzati su Trinidad per questo progetto si collegano a questo tema. $\mathrm{Si}$ veda anche Miller, D. 2011. Tales from Facebook. Cambridge: Polity.

$27 \mathrm{Si}$ veda Barthes, R. 1977. "Rhetoric of the Image», in Image-Music-Text. New York: Noonday, 32-37.

28 Si veda Hjorth, L. 2007. «Snapshot of Almost Contact: The Rise of Camera Phone Practices and a Case Study in Seoul, Korea», Continuum: Journal of Media \& Cultural Studies 21(2): 227-238, e Koskinen, I. 2006. «Managing Banality in Mobile Multimedia», in Pertierra, R. ed. The Social Construction and Usage of Communication Technologies: European and Asian Experiences. Singapore: Singapore University Press, 48-60.

29 Questo richiama "l'asserzione della verità" nella fotografia che si basa sull'indessicalità e l'accuratezza visiva delle fotografie. Si veda Gunning, T. 2004. "What's the Point of an Index? Or, Faking Photographs», NORDICOM Review 5 (1/2 September): 41.

30 Per un'elaborazione di questo argomento si veda Miller, D. 2016. «Photography in the Age of Snapchat», Anthropology and Photography. 1.

\section{Capitolo 12}

1 Ci è stato impossibile evitare alcune sovrapposizioni fra gli argomenti di questo e dei capitoli precedenti che trattano in prospettiva comparativa della vita online e offline. 
Dove ciò capita, abbiamo comunque usato per lo più esempi diversi.

2 Dumont, L. 1980. Homo Hierarchicus: The Caste System and its Implications. Chicago: University of Chicago Press, 45. Per una visione teorica più ampia si veda Morris, B. 1991. Western Conceptions of the Individual. Oxford: Berg.

3 Si veda per esempio Giddens, A. 1991a. Modernity and Self-identity: Self and Society in the Late Modern Age. Cambridge: Polity Press. 1991b. The Consequences of Modernity. Cambridge: Polity Press. Putnam, R. 2000. Bowling Alone: The Collapse and Revival of American Community. New York: London: Simon \& Schuster.

4 De Tocqueville, A. 1994 (1840). Democracy in America. London: David Campbell.

5 Si vedano in particolare i Capitoli 2 e 3 di Giddens, A. 1991a. Modernity and Selfidentity: Self and Society in the Late Modern Age. Cambridge: Polity Press. Nell'approccio di Giddens questa narrazione è essenziale al Sé per selezionare fra i significati molteplici e le scelte individuali offerti dalla tarda modernità.

6 Si tratta di un tema costante nei media occidentali, probabilmente una questione dominante nella stampa riguardo ai social media. Tipici casi relativi a Facebook sono: http://www.dailymail.co.uk/news/article-2419419/All-lonely-Facebookfriends-Study-shows-social-media-makes-MORE-lonely-unhappy-LESS-sociable. html; http://www.newyorker.com/tech/elements/how-facebook-makes-us-unhappy; http://www.theguardian.com/media-network/media-network-blog/2014/mar/13/ selfie-social- media-love-digital-narcassism

7 Per una teoria articolata sull'emergere di una società della rete si veda Castells, M. 2000. The Information Age: Economy, Society and Culture, 3 voll. Oxford: Blackwell. Anche Van Dijk, J.A.G.M. 1999. The Network Society: Social Aspects of New Media. London: Sage.

8 Rainie, L., Wellman, B. 2012. Networked. Cambridge, MA: The MIT Press.

9 Si veda la discussione di questo termine in Papacharissi, Z. 2010a. A Private Sphere: Democracy in a Digital Age. Cambridge: Polity Press, 138-144. Per una concezione simile del Sé in rete si veda Papacharissi, Z. ed. 2011. A Networked Self: Identity, Community and Culture on Social Network Sites. London: Routledge, e Cohen, J.E. 2012. Configuring the Networked Self: Law, Code, and the Play of Everyday Practice. New Haven, CT and London: Yale University Press.

10 Rainie, L., Wellman, B. 2012. Networked. Cambridge, MA: The MIT Press, 13.

11 Rainie, L., Wellman, B. 2012. Networked. Cambridge, MA: The MIT Press, 38-39.

12 Rainie, L., Wellman, B. 2012. Networked. Cambridge, MA: The MIT Press, 124-125. In particolare Rainie e Wellman sostengono che "Facebook è sia l'epitome del networking individuale - ogni persona è un individuo partecipante - sia del networked operating system nel suo insieme”. Rainie, L., Wellman, B. 2012. Networked. Cambridge, MA: The MIT Press, 144.

13 Si veda anche Baym, N. 2010. Personal Connections in the Digital Age. Cambridge: Polity Press, 6-12. Il lavoro argomenta a favore del potere delle persone di modellare le tecnologie senza per questo attenuare la socialità.

14 Fox, R. 1967. Kinship and Marriage. Harmondsworth: Penguin.

15 Mendleson, A., Papacharissi, Z. 2001. "Look at Us: collective narcissism in college student Facebook photo galleries», in Papacharissi, Z., ed. A Networked Self: Identity, community, and culture on social network sites. New York: Routledge, 251-273. Hogan, 
B., Wellman, B. 2015, «The relational self-portrait: selfies meet social networks», in Graham, M., Dutton, W., eds. Society and the Internet. Oxford: Oxford University Press. Nonostante questi titoli, il contenuto reale di entrambi gli articoli evidenzia le relazioni sociali in un modo adeguato agli argomenti presentati in questo capitolo.

16 In uno studio senza peer review condotto dalla rivista Time, Makati City e Pasig, nelle Filippine, emergono come la città con il più alto numero di selfie pro capite. Si può scaricare online: http://time.com/selfies-cities-world-rankings (scaricato il 20 luglio 2015).

17 Nicolescu, R. 2016. Social Media in Southeast Italy. London: UCL Press. Capitolo 5.

$18 \mathrm{Ci}$ sono centinaia di articoli su questo argomento. Si veda per esempio Andrejevic, M. 2002. "The Work of Being Watched», Critical Studies in Media Communication 19(2): 230-248. boyd, d. 2008. «Facebook’s Privacy Trainwreck: Exposure, Invasion, and Social Convergence», Convergence 14(1): 13-20. Etzioni, A. 1999. The Limits of Privacy. New York: Basic Books. Fuchs, C. 2012b. «The Political Economy of Privacy on Facebook», Television \& New Media 13(2): 139-159. Madden, M. 2012. Privacy Management on Social Media Sites: Most Users Choose Restricted Privacy Settings while Profile 'Pruning' and Unfriending People is on the Rise. Pew Research Center's Internet \& American Life Project. Trepte, S., Reinecke, L., eds. 2011. Privacy Online: Perspectives on Privacy and Self-Disclosure in the Social Web. New York: Springer. Utz, S., Kramer, N. 2009. "The Privacy Paradox on Social Network Sites Revisited: The Role of Individual Characteristics and Group Norms", Cyberpsychology: Journal of Psychosocial Research on Cyberspace 3(2).

19 Si veda boyd, d. 2008. «Facebook's Privacy Trainwreck: Exposure, Invasion, and Social Convergence», Convergence 14(1) 13-20.

20 Si veda la dettagliata analisi dei concetti cinesi di "pubblico" e "privato" in Chan, Y. K. 2000. «Privacy in the Family: Its Hierarchical and Asymmetric Nature», Journal of Comparative Family Studies 31(1): 1-17. Nicolescu, R. 2016. Social Media in Southeast Italy. London: UCL Press.

21 Nel caso di molti operai, i contatti sui social media che, per questo, arrivano a conoscere i loro sogni, timori, rimorsi o altri segreti, non conoscono i loro veri nomi o altri elementi della loro identità, quindi non possono danneggiare la loro reputazione on line.

22 Turkle, S. 2011. Alone Together: Why We Expect More from Technology and Less from Each Other. New York: Basic Books.

23 Hampton, K., Wellman, B. 2003. «Neighboring in Netville: How the Internet Supports Community and Social Capital in a Wired Suburb», City and Community 2(4): 277-311.

24 Per esempi dettagliati si veda Miller, D., Sinanan, J. 2014. Webcam. Cambridge: Polity Press, Capitolo 1.

25 Per una discussione di ciò che Miller definisce la 'Goldilocks Strategy', si veda Miller, D. 2016. Social Media in an English Village. London: UCL Press, Capitolo 4.

26 Per ulteriori approfondimenti degli aspetti temporali dei media per la comunicazione, del tipo sincronia/asincronia, si veda Baym, N. 2010. Personal Connections in the Digital Age. Cambridge: Polity Press, 7-8.

27 Si veda anche il Capitolo 7.

28 Hebdige, D. 1979. Subculture: The Meaning of Style. London: Methuen. 


\section{Capitolo 13}

1 Layard, R. 2011. Happiness: Lessons for a New Science. London: Penguin.

2 Rousseau, Jean-Jacques. 2010 (1754). Discourse on the Origin and Foundations of Inequality Among Men, Rosenblatt, H., trans. and ed. Boston, MA: Bedford/St. Martin's.

3 Durkheim, E. 1997 (1893). The Division of Labor in Society, Halls, W. D., trans. New York: The Free Press. Durkheim, E. 1979 (1897). Suicide: A Study in Sociology, Spaulding, J.A., Simpson, G., trans. New York: The Free Press.

4 James, W. 2012 (1902). The Varieties of Religious Experience. Boston, MA: Bedford/St. Martin's.

5 Weber, M. 2002 (1905). The Protestant Ethic and the Spirit of Capitalism. London: Penguin Books.

6 Locke, J. 1979 (1689). An Essay Concerning Human Understanding, Nidditch, P.H., ed. Oxford: Oxford University Press.

7 Comte, A. 1875. System of Positive Polity. London: Longmans, Green and Co.

8 Thin, N. 2005. «Happiness and the Sad Topics of Anthropology», WeD Working Paper 10. ESRC Research Group on Wellbeing in Developing Countries.

9 Anche se molti antropologi affrontano temi come il desiderio, il soddisfacimento e l'appartenenza, che possono svolgere un ruolo nella felicità individuale, il concetto di felicità raramente è stato teorizzato in modo esplicito.

10 Sen, A. 1999. Development As Freedom. Oxford: Oxford University Press.

11 Il PIL è stato a lungo ritenuto privo di significative relazioni con la "felicità", come evidenziato dall'inchiesta di Gallup sulla misurazione del benessere in base alla Cantril Self-Anchoring Scale. Si veda Cantril, H. 1965. The Pattern of Human Concerns. New Brunswick, NJ: Rutgers University Press.

12 "Gross National Happiness". 2008. Centre for Bhutan Studies and GNH Research, http://www.grossnationalhappiness.com/gnhIndex/intruductionGNH.aspx.

13 Helliwell, J., Layard, R., Sachs, J., eds. 2015. World Happiness Report 2015. New York: Sustainable Development Solutions Network.

14 "Human Development Index." 2014. United Nations Development Programme, http:// hdr.undp.org/en/statistics/hdi/

15 Maslow, A. H. 1943. "A Theory of Human Motivation», Psychological Review 50(4): 370-396.

16 Helliwell, J., Layard, R., Sachs, J., eds. 2015. World Happiness Report 2015. New York: Sustainable Development Solutions Network. 13.

17 Thin, N. 2005. «Happiness and the Sad Topics of Anthropology», WeD Working Paper 10. ESRC Research Group on Wellbeing in Developing Countries.

18 Wali, A. 2012. "A Different Measure of Well-Being" Vital Topics Forum. Johnston, B.R., ed. American Anthropologist 114(1): 12.

19 Tandoc, E.C. Jr., Ferruci, P., Du y, M. 2015. «Facebook Use, Envy, and Depression Among College Students: Is Facebooking Depressing?», Computers in Human Behavior 43: 139-146.

20 Kross, E., Verduyn, P., Demiralp, E., Park, J., Lee, D. S., Lin, N., Shablack, H., Jonides, J., Ybarra, O. 2013. «Facebook Use Predicts Declines in Subjective Well-Being in Young 
Adults», PLoS ONE 8(8): e69841.

21 Muise, A., Christofides, E., Desmarais, S. 2009. "More Information Than You Ever Wanted: Does Facebook Bring Out the Green-eyed Monster of Jealousy?», Cyberpsychological Behavior 12(4): 441-444.

22 Tiggeman, M., Slater, A., 2013. "NetGirls: The Internet, Facebook and Body Image Concern in Adolescent Girls», International Journal of Eating Disorders 46(6): 630-633.

23 O'Keeffe, G.S., Clarke-Pearson, K. 2011. "Clinical Report - The Impact of Social Media on Children, Adolescents, and Families», American Academy of Pediatrics, http://pediatrics.aappublications.org/content/early/2011/03/28/peds.2011-0054.full. pdf + html?ijkey=76f29031adb1f95a04cca23436b5ccdebfd5cd9f.

24 O'Keeffe, G. S., Clarke-Pearson, K. 2011. «Clinical Report-The Impact of Social Media on Children, Adolescents, and Families», American Academy of Pediatrics, http://pediatrics.aappublications.org/content/early/2011/03/28/peds.2011-0054.full. pdf + html?ijkey=76f29031adb 1 f95a04cca23436b5ccdebfd5cd9f.

25 Tandoc, E.C. Jr., Ferruci, P., Duffy, M. 2015. «Facebook Use, Envy, and Depression Among College Students: Is Facebooking Depressing?», Computers in Human Behavior 43: 139-146.

26 Sagioglou, C., Greitemeyer, T. 2014. «Facebook's Emotional Consequences: Why Facebook Causes a Decrease in Mood and Why People Still Use It», Computers in Human Behavior 35: 359-363.

27 Lauren, A., Jelenchick, J., Eickho, C., Moreno, M.A. 2013. «Facebook Depression? Social Networking Site Use and Depression in Older Adolescents", Journal of Adolescent Health 52(1): 128-130.

28 Hiscott, R. 2014. «Why You Feel Terrible After Spending Too Much Time On Facebook», The Huffington Post http://www.huffingtonpost.com/2014/07/17/facebook-study_n_ 5595890.html, (accesso 18 luglio 2014).

29 Valenzuela, S., Park, N., Kee, K. F. 2009. «Is There Social Capital in a Social Network Site?: Facebook Use and College Students' Life Satisfaction, Trust and Participation», Journal of Computer-Mediated Communication 14(4): 875-901.

30 Kramer, A.D., Guillory, J.E., Hancock, J.T. 2014. «Experimental Evidence of MassiveScale Emotional Contagion through Social Networks», Proceedings of the National Academy of Sciences 111(24): 8788-8790.

31 Miller e Slater indicano che lo stesso internet (e i social media per estensione) non esiste precedentemente al suo uso, ma si crea mediante le azioni individuali degli utenti, in gran parte nel modo in cui i linguisti seguono la nozione di J.L. Austin di atto performativo, che suggerisce che gli atti illocutivi non solo descrivono una realtà data, ma anche trasformano la realtà sociale che stanno descrivendo. Come tali, le piattaforme di social media sono semplicemente l'ambiente in cui il contenuto può mutare ciò che è il medium particolare e ciò che significa per gli utenti. Si veda Miller, D., Slater, D. 2000. The Internet: An Ethnographic Approach. Oxford: Berg. Austin, J.L. 1962. How to Do Things with Words. Oxford: Oxford University Press.

$32 \mathrm{Si}$ veda la Figura 4.26 per i risultati e una spiegazione dell'inchiesta.

33 Per i siti a Trinidad e in Inghilterra si usava l'espressione "più felice", come si usava in India dove "felice" è parte del tanglish (tamil e inglese) usato comunemente. È stato 
tradotto come 'más feliz' in Chile, 'mais feliz' in Brasile, e 'più felice' in Italia. In Turchia si usava il termine 'mutlu', la più comune fra le varie parole che si possono tradurre con "felice”. In Cina si usava il concetto di 'kuaile' (felice, gioioso, sorridente), opposto a 'xingfu' che mette in evidenza un senso "più profondo" di felicità, che riflette il fatto che i social media sono innanzitutto una fonte di divertimento.

34 Appadurai, A. 2004. "The Capacity to Aspire: Culture and the Terms of Recognition», in Rao, V., Walton, M., eds. Culture and Public Action. Stanford, CA: Stanford University Press, 59-84.

35 Wang, X. 20016. Social Media in Industrial China. London: UCL Press.

36 Haynes, N. 2016. Social Media in Northern Chile. London: UCL Press.

37 Frazier, L. 2007. Salt in the Sand: Memory, Violence, and the Nation-State in Chile, 1890 to the Present. Durham, NC: Duke University Press.

38 Spyer, J. 2017. Social Media in Emergent Brazil. London: UCL Press.

39 McDonald, T. 2016. Social Media in Rural China. London: UCL Press.

40 Wang, R.R. 2002. "Globalizing the Heart of the Dragon: The Impact of Technology on Confucian Ethical Values», Journal of Chinese Philosophy 29(4): 553-569.

41 Rainey, L.D. 2010. Confucius \& Confucianism: The Essentials. Malden, MA: WileyBlackwell. 16.

42 Miller, D. 2011. Tales from Facebook. Cambridge: Polity Press.

43 Sinanan, J. 2017. Social Media in Trinidad. London: UCL Press.

44 YY è un social network cinese basato sul video, con più di 300 milioni di utenti.

45 A differenza degli operai migranti, che usano raramente il loro vero nome e vere fotografie su QQ, mentre tendono a creare un mondo finto online usando immagini raccolte da internet, la classe media cinese preferisce sempre di più WeChat a QQ. Su WeChat la percentuale di nomi reali è molto più alta che su QQ.

46 Nicolescu, R. 2016. Social Media in Southeast Italy. London: UCL Press.

47 Costa, E. 2016. Social Media in Southeast Turkey. London: UCL Press.

48 Venkatraman, S. 2017. Social Media in South India. London: UCL Press.

49 Hogan, B. 2010. "The Presentation of Self in the Age of Social Media: Distinguishing Performances and Exhibitions Online», Bulletin of Science, Technology, and Society 30(6): 377-386.

50 Si veda la Figura 4.26.

\section{Capitolo 14}

1 Miller, D. 2014. «Hospices: The Potential for New Media»: http://www.ucl.ac.uk/ anthropol-ogy/people/academic_staff/d_miller/mil-28

2 Miller, D., Sinanan, J. 2014. Webcam. Cambridge: Polity Press, Capitolo 1.

3 Miller, D., Sinanan, J. 2014. Webcam. Cambridge: Polity Press, Capitolo 1.

4 Van Dijck, J. 2007. Mediated Memories in the Digital Age. Stanford, CA: Stanford University Press.

5 Thompson, C. 2013. Smarter Than You Think. London: Penguin. 
6 Miller, D. 2015. «Photography in the Age of Snapchat», Anthropology and Photography, 1.

7 Miller, D., Sinanan, J. 2014. Webcam. Cambridge: Polity Press, Capitolo 1.

8 Si veda per esempio la Figura 4.9.

9 Jeffrey, R., Doron, A. 2013. The Great Indian Phone Book. London: Hurst.

10 Wallis, C. 2011. «New Media Practices in China: Youth Patterns, Processes, and Politics», International Journal of Communication 5: 406-436.

11 Secondo un rapporto nazionale, l' $83,4 \%$ di tutti gli utenti di internet in Cina usa i cellulari per l'accesso a internet. China International Network Information Center. 2014. $34^{\text {th }}$ Statistical Report on Internet Development in China. http://www1.cnnic.cn/IDR/ ReportDownloads/201411/P020141102574314897888.pdf (accesso 28 marzo 2015)

12 Per la prevista crescita di 20 volte nei prossimi cinque anni si veda http://www.theguardian. $\mathrm{com} /$ world/2014/jun/05/internet-use-mobile-phones-africa-predicted-increase-20-fold

13 The Economist. 28 febbraio 2015.

14 Graham, M. 2014. «Internet Geographies: Data Shadows and Digital Divisions of Labour», in Graham, M., Dutton, W., eds. Society and the Internet. Oxford: Oxford University Press, 99-116.

15 The Economist. 13 settembre 2014.

16 In tale connessione, vale la pena sottolineare la popolarità crescente fra i cinesi urbani che utilizzano siti di shopping online che offrono grandi firme a prezzi fissi, per esempio JD.com e Tmall, che allo stesso modo considerano l'esigenza di un'interazione al fine di fare acquisti come una notevole seccatura.

17 Per esempio Simmel, G. 1968. The Conflict in Modern Culture and other Essays. New York: Teachers College Press.

18 Si veda Miller, D., Horst, H. 2012. «Introduction», in Horst, H., Miller, D., eds. Digital Anthropology. London: Berg: 4-11.

19 Malaby, T. 2009. Making Virtual Worlds. Ithaca, NY: Cornell University Press.

20 Coleman, G. 2010. «Ethnographic Approaches to Digital Media», Annual Review of Anthropology 39: 487-505.

21 Kelty, C. 2008. Two Bits: The Cultural Significance of Free Software. Durham, NC: Duke University Press.

22 Horst, H., Miller, D., eds. 2012. Digital Anthropology. London: Berg, 28-30.

23 http://blogs.ucl.ac.uk/global-social-media/2013/02/14/my-whatsapp-field-trip/

24 Si veda la discussione sulla "novitä" dei social media in Gershon, I., Bell, J.A. 2013. "Introduction: The Newness of New Media», Culture, Theory and Critique 54(3): 259-264.

25 Si veda Wang, X. 2016. Social Media in Industrial China. London: UCL Press, Capitolo 2.

26 Sinanan, J. 2017. Social Media in Trinidad. London: UCL Press.

27 Wang, X. 2016. Social Media in Industrial China. London: UCL Press. 


\section{Bibliografia}

Aarsand, P.A. 2008. «Frame switches and identity performances: alternating between online and offline», Text \& Talk 28(2): 147-165.

Adorno, T.W., Horkheimer, M. 2002. Dialectic of Enlightenment. Stanford, CA: Stanford University Press.

Adorno, T.W. 1991. "Free time», in Bernstein, J.M., ed. The Culture Industry: Selected essays on mass culture, 162-170.

Akdeniz, Y., Altiparmak, K. 2008. «Internet: restricted access: a critical assessment of Internet content regulation and censorship in Turkey», http://privacy.cyber-rights.org.tr/?page_id=256

Akdenizli, B., ed. 2015. Digital Transformations in Turkey: Current Perspectives in Communication Studies. Lanham, MD: Lexington Books.

Akinnaso, F.N. 1992. "Schooling, Language and Knowledge in Literate and Nonliterate societies", Comparative Studies in Society and History 34: 68-109.

Alev Degim, I., Johnson, J., Fu, T., eds. 2015. Interpersonal Interactions Across Borders. Amsterdam: Institute of Network Cultures.

Andrejevic, M. 2002. "The work of being watched», Critical Studies in Media Communication 19(2): 230-248.

Appadurai, A. 2004. «The Capacity to Aspire: Culture and the Terms of Recognition», in Rao, V., Walton, M., eds. Culture and Public Action. Stanford, CA: Stanford University Press, 59-84.

Askew, K., Wilk, R. 2002. Anthropology of Media: A Reader. Oxford: Blackwell.

Austin, J.L. 1962. How to Do Things with Words. Oxford: Oxford University Press.

Baba, M L. 1998. "The anthropology of work in the Fortune 1000: a critical retrospective»,

Anthropology of Work Review 18(4):17-28.

Baker, L.D. 1998. From Savage to Negro: Anthropology and the Construction of Race. Los Angeles, CA: University of California Press.

Banks, M., Morphy, H., eds. 1997. Rethinking Visual Anthropology. New Haven, CT: Yale University Press.

Barendregt, B. 2012. «Diverse Digital Worlds», in Horst, H., Miller, D., 
eds. Digital Anthropology. London: Berg, 203-224.

Bargh, J.A., et. al. 2002. "Can You See the Real Me? Activation and Expression of the "True Self"

on the Internet», Journal of Social Issues 58(1): 33-48.

Barlow, J., 1996. "A Declaration of the Independence of Cyberspace». Electronic Frontier Foundation 8 February 1996. https://www.eff.org/it/ cyberspace-independence (accesso 7 luglio 2015).

Barthes, R. 1977. «Rhetoric of the Image», in Image-Music-Text. New York: Noonday, 32-37.

Bateson, G., Mead, M. 1942. "Balinese character. A photographic analysis", Special Publications of the New York Academy of Science: 17-92.

Baym, N. 1999. Tune In, Log On: Soaps, Fandom, and Online Community. New York: Sage.

- 2010. Personal Connections in the Digital Age. Cambridge: Polity Press.

Bernal, V. 2014. Nation as Network. Chicago, IL: University of Chicago Press.

Bernard, H.R. 2011. Research methods in anthropology: Qualitative and quantitative approaches. Lanham, MD: Altamira Press.

Bitz, M. 2004. "The comic book project: The lives of urban youth», Art Education 57(2): 33-46.

- 2010. When Commas Meet Kryptonite: Classroom Lessons from the Comic Book Project. Language and Literacy Series. New York: Teachers College Press.

Boase, J., Wellman, B. 2006. «Personal Relationships: On and Off the Internet», in Perlman, D., Vangelisti, A., eds. Handbook of Personal Relations. Cambridge: Cambridge University Press.

Boellstorff, T., Nardi, B., Pearce, C., Taylor, T. 2012. Ethnography and Virtual Worlds: A Handbook of Method. Princeton, NJ: Princeton University Press.

Boellstorff, T. 2008. Coming of Age in Second Life. Princeton, NJ: Princeton University Press.

Borofsky, R. 1987. Making history: Pukapukan and anthropological constructions of knowledge. Cambridge: Cambridge University Press.

Bourdieu, P., Bourdieu, M.C. 2004. "The peasant and photography», Ethnography 5(4): 601-616.

Bourdieu, P., Wacquant, L. 1992. "Symbolic Violence», in An Invitation to Reflective Sociology. Chicago: University of Chicago Press, 167-173. 
Bourdieu, P., Whiteside, S. 1996. Photography: A middle-brow art. Stanford, CA: Stanford University Press.

Bourdieu, P. 1972. Outline to a Theory of Practice. Cambridge: Cambridge University Press.

- 1984. Distinction: A social critique of the judgment of taste. Cambridge, MA: Harvard University Press.

boyd, d., Ellison, N.B. 2007. «Social Network Sites: Definition, History and Scholarship", Journal of Computer-Mediated Communication 13(1): 211-230.

boyd, d. 2008. «Facebook's Privacy Trainwreck: Exposure, Invasion, and Social Convergence», Convergence 14(1): 13-20.

- 2013. "White Flight in Networked Publics? How Race and Class Shaped American Teen Engagement with Myspace and Facebook», in Nakamura, L., Chow-White, P., eds. Race after the Internet. New York: Routledge, 203-222.

- 2014. It's complicated: The social lives of networked teens. New Haven, CT; London: Yale University Press.

Brants, K. 2005. Guest editor's introduction: «The Internet and the Public Sphere», Political Communication 22(2): 143-146.

Broadbent, S. 2011. L'Intimité au Travail. Paris: Fyp Editions.

— 2012. "Approaches to Personal Communication», in Horst, H., Miller, D., eds. Digital Anthropology. London, Oxford: Berg, 127-145.

Brubaker, R., Cooper, F. 2000. "Beyond "Identity" Theory and Society 29: 1-47.

Bubel, C.M. 2008. «Film Audiences as Overhearers», Journal of Pragmatics 40: 55-71.

Buckingham, D. 2003. Media education: literacy, learning and contemporary culture. Cambridge: Polity Press.

Butler, J. 1990. Gender Trouble. New York: Routledge.

Cantril, H. 1965. The Pattern of Human Concerns. New Brunswick, NJ: Rutgers University Press.

Carr, N. 2011. The Shallows: What the Internet Is Doing to Our Brains. New York: W. W. Norton \& Company.

Castells, M. 1996. The Rise of the Network Society. The Information Age: Economy, Society and Culture, Vol. I. Oxford: Blackwell.

- 1997. The Power of Identity. The Information Age: Economy, Society and Culture, Vol II. Oxford, UK: Blackwell. 
- 2000. The Information Age: Economy, Society and Culture, Updated edition, 3 volumes. Oxford: Blackwell.

Chadwick, A., Howard, P.N. 2010. Routledge Handbook of Internet politics. Oxford: Taylor \& Francis.

Chadwick, A. 2003. «Bringing E-Democracy Back In: Why it Matters for Future Research on E-Governance», Social Science Computer Review 21(4): 443-455.

Chambers, D. 2013. Social Media and Personal Relationships: Online Intimacies and Networked Friendship. Basingstoke: Palgrave Macmillan.

Chan, Y. K. 2000. «Privacy in the Family: Its Hierarchical and Asymmetric Nature», Journal of

Comparative Family Studies 31(1): 1-17.

Chen, W., Wellman, B. 2004. "The global digital divide within and between countries", IT \& Society 1(7): 39-45.

Chu, J.Y. 2010. Cosmologies of Credit: Transnational Mobility and the Politics of Destination in China. Durham, NC: Duke University Press.

Clark, L.S. 2013. The Parent App. Oxford: Oxford University Press.

Clay. S. 2011. "The Political Power of Social Media», Foreign Affairs 90: 128-141.

Cohen, J.E. 2012. Configuring the Networked Self: Law, Code, and the Play of Everyday Practice. New Haven, CT and London: Yale University Press. Coleman, G. 2010. "Ethnographic Approaches to Digital Media», Annual Review of Anthropology 39: 487-505.

- 2012a. Coding Freedom: The Ethics and Aesthetics of Hacking. Princeton, NJ: Princeton University Press.

— 2012b. «Phreaks, Hackers, and Trolls: The politics of Transgression and Spectacle», in Maniberg, M., ed. The Social Media Reader. New York: New York University Press, 99-119.

- 2014. Hacker, hoaxer, whistleblower, spy: The many faces of Anonymous. London; Brooklyn, NY: Verso.

Comte, A. 1875. System of Positive Polity. London: Longmans, Green and Co.

Costa, E. 2016. Social Media in Southeast Turkey. London: UCL Press. Couldry, N. 2015. "Social Media: Human Life», Social Media + Society $1(2)$.

Cross, M. 2011. Bloggerati, Twitterati: How Blogs and Twitter are transforming popular culture. Santa Barbara, CA: Praeger. 
Cruz, E.G., Meyer, E. 2012. "Creation and Control in the Photographic Process: iPhones and the Emerging Fifth Moment of Photography», Photographies 5(2).

Culnan, M.J., McHugh, P.J., Zubillaga, J.I. 2010. "How Large US Companies Can Use Twitter and Other Social Media to Gain Business Value», MIS Quarterly Executive 9(4): 243-259.

Dahlberg, L. 2001. «The Internet and Democratic Discourse: Exploring the Prospects of Online Deliberative Forums Extending the Public Sphere», Information, Communication \& Society 4(4): 615-633.

Dalsgaard, S. 2008. «Facework on Facebook: The presentation of Self in Virtual Life and its Role in the US Elections", Anthropology Today 24(6): 8-12.

David, G. 2010. "Camera Phone Images, Videos and Live Streaming: A Contemporary Visual Trend», Visual Studies 25(1): 89-98.

Dawes, S.S. 2008. "The evolution and continuing challenges of e-governance», Public+Administration Review 68(1): 86-102.

De Neve, G. 2005. The everyday Politics of Labour: Working Lives in India's Informal Economy. Oxford: Berghahn Books.

De Tocqueville, A. 1994 (1840). Democracy in America. London: David Campbell.

Dumont, L. 1980. Homo Hierarchicus: The Caste System and its Implications. Chicago: University of Chicago Press.

Durkheim, E. 1979 (1897). Suicide: A Study in Sociology, Spaulding, J.A., Simpson, G., trans. New York: The Free Press.

- 1997 (1893). The Division of Labor in Society, Halls, W. D., trans. New York: The Free Press.

Edwards, E. 1992. Anthropology and Photography 1860-1929. Royal Anthropological

Institute.

Ellison, N.B., Steinfield, C., Lampe, C. 2007. "The Benefits of Facebook "Friends": Exploring the Relationship between College Students' Use of Online Social Networks and Social Capital», Journal of ComputerMediated Communication, 12(3), 1143-1168.

Fenton, N., Barassi, V. 2011. "Alternative Media and Social Networking Sites: The Politics of Individuation and Political Participation», The Communication Review 14(3): 179-196.

Fong, V.L. 2004. Only Hope: Coming of Age under China's One-child Policy. 
Stanford, CA: Stanford University Press Fountain, J. E. 2001. Building the Virtual State: Information Technology and Institutional Change. Washington, DC: Brookings Institution Press.

Fox, R. 1967. Kinship and Marriage. Harmondsworth: Penguin.

Frazier, L. 2007. Salt in the Sand: Memory, Violence, and the Nation-State in

Chile, 1890 to the Present. Durham, NC: Duke University Press.

Fu, K.W., Chan, C.H. 2015. "Networked Collective Action in the 2014 Hong Kong Occupy Movement: Analysing a Facebook Sharing Network», International Conference on Public Policy, ICPP 2015.

Fuchs, C. 2012a. «Social media, riots, and revolutions», Capital \& Class 36(3): 383-391.

— 2012b. "The Political Economy of Privacy on Facebook», Television \& New Media 13(2): 139-59.

- 2013. Social Media: A Critical Introduction. London: Sage.

Fuller, C. J., Narasimhan, H. 2007. «Information Technology Professionals and the New-rich Middle Class in Chennai (Madras)", Modern Asian Studies 41(1): 121-150.

Gardner, R. 1971. The Nuer (Director of the film).

Gerbaudo, P. 2012. Tweets and the streets: Social media and contemporary activism. London: Pluto Press.

Gershon, I., Bell, J.A. 2013. «Introduction: The Newness of New Media», Culture, Theory and Critique 54(3): 259-264.

Gershon, I. 2010. Breakup 2.0: Disconnecting Over New Media. Cornell: Cornell University Press.

— 2011. «Un-Friend My Heart: Facebook, Promiscuity and Heartbreak in a Neoliberal Age», Anthropological Quarterly 84(4): 865-894.

Gibbs, M., Meese, J., Arnold, M., Nansen, B., Carter, M. 2015. «\#Funeral and Instagram: Death, Social Media, and Platform Vernacular", Information, Communication \& Society 18(3): 255-268.

Giddens, A. 1991a. Modernity and Self-identity: Self and Society in the Late Modern Age. Cambridge: Polity Press.

- 1991b. The Consequences of Modernity. Cambridge: Polity Press.

Gingrich, A., Fox, R. G., eds. 2002. Anthropology, By Comparison. London: Routledge.

Ginsburg, F., Abu-Lughod, L., Larkin, B., eds. 2002. Media Worlds. Berkeley, CA: University of California Press.

Ginsburg, F. 1995. «Mediating Culture: Indigenous Media, Ethnographic 
Film, and the Production of Identity", Fields of Vision: Essays in Film Studies, Visual Anthropology, and Photography. Berkeley,CA: University of California Press.

Godelier, M. 2012. The Metamorphosis of Kinship. London: Verso.

Goffman, E. 1959. The Presentation of the Self in Everyday Life. Garden City, NY: Anchor Books.

- 1975. Frame Analysis. Harmondsworth: Penguin.

Goldfarb, A., Tucker, C. 2011. «Online Display Advertising: Targeting and Obtrusiveness", Marketing Science 30(3): 389-404.

Graham, M., Dutton, W., eds. 2014. Society and the Internet. Oxford: Oxford University Press.

Graham, M. 2014. "Internet Geographies: Data Shadows and Digital Divisions of Labour", in Graham, M., Dutton, W., eds. Society and the Internet. Oxford: Oxford University Press, 99-116.

Gray, M.L. 2009. Out in the Country: Youth, Media, and Queer Visibility in Rural America. New York: New York University Press.

Greschke, H. 2012. Is There a Home in Cyberspace?: The Internet in Migrants' Everyday Life and the Emergence of Global Communities. London: Routledge.

Grint, K. 2005. The sociology of work: introduction. Cambridge: Polity Press. Gross National Happiness. Centre for Bhutan Studies and GNH Research, http://www.grossna- tionalhappiness.com/gnhIndex/intruductionGNH. aspx, 2008.

Gunning, T. 2004. «What's the Point of an Index? Or, Faking Photographs», NORDICOM Review 5

(1/2 September): 41.

Habermas, J. 1969. The Structural Transformation of the Public Sphere: An Inquiry into a Category of Bourgeois Society. Cambridge, MA: The MIT Press.

Hampton, K.N., Rainie, L., Lu, W., Dwyer, M., Shin, I., Purcell, K. 2014. "Social Media and the "Spiral of Silence"», Pew Research Center, Washington, DC. http:// www.pewinternet.org/2014/08/26/ social-media-and-the-spiral-of-silence/.

Hampton, K., Wellman, B. 2003. "Neighboring in Netville: How the Internet Supports Community and Social Capital in a Wired Suburb», City and Community 2(4): 277-311.

Hanna, R., Rohm, A., Crittenden, V. L. 2010. «We're All Connected: The 
Power of the Social Media Ecosystem», Business Horizons, 54(3): 265-273. Haraway, D. 1991. "A Cyborg Manifesto: Science, Technology and Social Feminism in the Late Twentieth Century», in Simians, Cyborgs and Women: The reinvention of Nature. New York: Routledge.

Hasebrink, U., Livingstone, S., Haddon, L. 2008. Comparing Children's Online Opportunities and Risks across Europe: Cross-National Comparisons for EU Kids Online. $2^{\text {nd }}$ edition. http:// eprints.lse.ac.uk/24368/.

Haynes, N. 2016. Social Media in Northern Chile. London: UCL Press. Hebdige, D. 1979. Subculture: The Meaning of Style. London: Methuen.

Heeks, R. 2001. «Building e-governance for development: A framework for national and donor action» Institute for Development Policy and Management, University of Manchester.

Helliwell, J., Layard, R., Sachs, J., eds. 2015. World Happiness Report 2015. New York: Sustainable Development Solutions Network.

Hine, C. 2000. Virtual ethnography. London: Sage.

Hiscott, R. 2014. "Why You Feel Terrible After Spending Too Much Time On Facebook», The Huffington Post, http://www.huffingtonpost. com/2014/07/17/facebook-study_n_5595890.html, (accesso 18 luglio 2014).

Hjorth, L., Hendry, N. 2015. «A Snapshot of Social Media: Camera Phone Practices», Social Media + Society 1(1): 1-3.

Hjorth, L. 2007. «Snapshot of Almost Contact: The Rise of Camera Phone Practices and a Case Study in Seoul, Korea», Continuum: Journal of Media \& Cultural Studies 21(2): 227-238.

- 2009. "Gifts of Presence: A Case Study of a South Korean Virtual Community, Cyworld's Mini-Hompy», in Goggin, G., McLelland, M., eds. Internationalising the Internet. London: Routledge, 237-251.

- 2010. 'The Game of Being Social: Web 2.0, Social Media, and Online Games.' Iowa Journal of Communication 42(1): 73-92.

Ho, C. 2000. "Popular Culture and the Aestheticization of Politics: Hegemonic Struggle and Postcolonial Nationalism in Trinidad Carnival», Transforming Anthropology 9(1): 3-18.

Hogan, B., Wellman, B. 2014. "The relational self-portrait: selfies meet social networks», in Graham, M., Dutton, W., eds. Society and the Internet. Oxford: Oxford University Press.

Hogan, B. 2010. "The Presentation of Self in the Age of Social Media: Distinguishing Performances and Exhibitions Online», Bulletin of 
Science, Technology, and Society 30(6): 377-386.

Holmström, M. 1976. South Indian Factory Workers: Their Life and Their World. Cambridge: Cambridge University Press.

Holy, L., ed. 1987. Comparative Anthropology. Oxford and New York: Blackwell.

Horst, H., Miller, D., eds. 2012 Digital Anthropology. London: Berg.

Hull, G., Schultz, K. 2002. School's Out: Bridging Out-of-school Literacies with Classroom Practice. New York, NY: Teachers College Press.

"Human Development Index." 2014. United Nations Development Programme, http://hdr.undp.org/en/statistics/hdi/.

Hunsiger, J., Senft, T., eds. 2014. The Social Media Handbook. New York: Routledge.

Hussain, M.M., Howard, P.N. 2012. «Opening Closed Regimes: Civil Society, Information Infrastructure, and Political Islam» in Anduiza, E., Jensen, M., Jorba, L., eds. Comparing Digital Politics: Civic Engagement and Political Participation. Cambridge: Cambridge University Press.

— 2013. "What Best Explains Successful Protest Cascades? Icts and the Fuzzy Causes of the Arab Spring», International Studies Review 15(1): 48-66.

Ito, M. et al. 2010. Hanging Out, Messing Around, and Geeking Out. Cambridge, MA: The MIT Press.

Ito, M., Horst, H., Bittanti, M., boyd, d., Herr-Stephenson, B., Lange, P.G., Pascoe, C.J., Robinson, L. 2008. Living and Learning with New Media: Summary of Findings from the Digital Youth Project. Cambridge, MA: The MIT Press.

Ito, M., Okabe, D. 2005. «Intimate visual co-presence», Position paper for the Seventh International Conference on Ubiquitous Computing, Tokyo.

Ito, M. 2005. "Mobile phones, Japanese youth, and the re-placement of social contact», Mobile Communication: 131-48.

James, W. 2012 (1902). The Varieties of Religious Experience. Boston, MA: Bedford/St. Martin's.

Jankowiak, W. 2002. «Proper Men and Proper Women: Parental Affection in the Chinese Family", in Brownell, S., Wasserstrom, J., eds. Chinese Femininities/Chinese Masculinities: A Reader. Berkeley, CA: University of California Press, 361-381.

Jeffrey, R., Doron, A. 2013. The Great Indian Phone Book. London: Hurst. Johnson, S. 2001. Emergence: The Connected Lives of Ants, Brains, Cities, 
and Software. New York: Scribner

Jones, G., Schieflin, B., Smith, R. 2011. "When Friends Who Talk Together Stalk Together: Online Gossip as Meta Communication", in Thurlow, C., Mroczek, K., eds. Digital Discourse: Language in the New Media. Oxford: Oxford University Press: 26-47.

Jordan, A. 2003. Business Anthropology. Long Grove: Waveland Press.

Jussila, J., Kärkkäinen, H., Leino, M. 2011. «Benefits of Social Media in

Business-to-Business Customer Interface in Innovation", Proceedings of the $15^{\text {th }}$ International Academic MindTrek Conference: Envisioning Future Media Environments: 167-174.

Kelly, K. 1994. Out of Control: The Rise of Neo-biological Civilization. Reading, MA: Addison-Wesley.

Kelty, C. 2008. Two Bits: The Cultural Significance of Free Software. Durham, NC: Duke University Press.

Kimble, C., Hildreth, P.M., Bourdon, I. eds. 2008. Communities of practice: creating learning environments for educators. Charlotte, NC: Information Age.

King, G., Pan, J., Roberts, M.E. 2013. «How Censorship in China Allows Government Criticism but Silences Collective Expression», American Political Science Review 107(2): 326-343.

Kipnis, A.B. 2011. Governing Educational Desire: Culture, Politics, and Schooling in China. Chicago, IL: University of Chicago Press

Kleine, D. 2013. Technologies of Choice: ICT's Development and the Capabilities Approach. Cambridge, MA: The MIT Press.

Klotz, R. J. 2004. The politics of Internet communication. Lanham: Rowman \& Littlefield.

Koskinen, I. 2006. «Managing Banality in Mobile Multimedia», in Pertierra, R. ed. The Social Construction and Usage of Communication Technologies: European and Asian Experiences. Singapore: Singapore University Press, 48-60.

Kramer, A.D., Guillory, J.E., Hancock, J.T. 2014. «Experimental Evidence of Massive-Scale Emotional Contagion through Social Networks", Proceedings of the National Academy of Sciences 111(24): 8788-8790.

Krasnova, H., Wenniger,H., Widjaja,T., Buxmann, P. 2013. «Envy on Facebook: A Hidden Threat to Users' Life Satisfaction?», Wirtschaftsinformatik Proceedings: Paper 92.

Kross, E., Verduyn, P., Demiralp, E., Park, J., Lee, D. S., Lin, N., Shablack, 
H., Jonides, J., Ybarra, O. 2013. "Facebook Use Predicts Declines in Subjective Well-Being in Young Adults», PLoS ONE 8(8): e69841.

Kumar, N. 2014. «Facebook for Self-empowerment? A Study of Facebook Adoption in Urban India», New Media \& Society 16(7): 1122-1137.

Kuntsman, A., Stein, R. 2015. Digital Militarism: Israel's Occupation in the Social Media Age. Stanford, CA: Stanford University Press.

Landzelius, K., ed. 2006. Native on the Net. London: Routledge.

Lange, P. 2014. Kids on YouTube. Walnut Creek, CA: Left Coast Press.

Lasén, A., Gómez-Cruz, E. 2009. «Digital photography and picture sharing: redefining the public/private divide», Knowledge, Technology \& Policy 22(3): 205-215

Lauren, A., Jelenchick, J., Eickho, C., Moreno, M.A. 2013. «Facebook Depression? Social Networking Site Use and Depression in Older Adolescents", Journal of Adolescent Health 52(1): 128-130.

Lave, J. 1993. Situated Learning: Legitimate Peripheral Participation. Cambridge: Cambridge University Press.

Layard, R. 2011. Happiness: Lessons for a New Science. London: Penguin.

Lee, K., Kim, J.H., Woo, W.T., eds. 2009. Power and Sustainability of the Chinese State. New York: Routledge.

Lenhart, A. 2015. Teens, Social Media \& Technology Overview. http://www. pewinternet.org/ 2015/04/09/teens-social-media-technology-2015/ (accesso 9 maggio 2015).

Levine, G. ed. 1971. Georg Simmel on Individuality and Social Forms. Chicago, IL: Chicago University Press.

Lim, M. 2012. "Clicks, Cabs, and Coffee Houses: Social Media and Oppositional Movements in Egypt, 2004-2011», Journal of Communication 62(2): 231-248.

- 2013. "Many Clicks but Little Sticks: Social Media Activism in Indonesia», Journal of Contemporary Asia 43(4): 636-657.

Lindtner, S. et al. 2011. "Towards a Framework of Publics: Re-encountering Media Sharing and its User», ACM Transactions on Computer-Human Interaction (TOCHI) 18(2): 5.

Livingstone, S., Mascheroni, G., Ólafsson, K., Haddon, L., with EU Kids Online and Net Children Go Mobile. 2014. "Children's Online Risks and Opportunities: Comparative Findings from EU Kids Online and Net Children Go Mobile», novembre 2014. http:// eprints.lse.ac.uk/ 60513/.

Livingstone, S., Sefton-Green, J. 2016. The Class: Connections and discon- 
nections in the digital age. New York: New York University Press.

Livingstone, S. 2008. "Taking Risky Opportunities in Youthful Content Creation: Teenagers' Use of Social Networking Sites for Intimacy, Privacy and Self-expression", New Media \& Society 10: 393-411.

- 2009. Children and the Internet. Cambridge: Polity Press.

Locke, J. 1979 (1689). An Essay Concerning Human Understanding. Nidditch, P.H, ed. Oxford: Oxford University Press.

Lomborg, S. 2014. Social Media, Social Genres: Making Sense of the Ordinary. London: Routledge.

MacDougall, D. 2005. The corporeal image: Film, ethnography, and the senses. Princeton, NJ: Princeton University Press.

MacKinnon, R. 2008. «Flatter World and Thicker Walls? Blogs, Censorship and Civic Discourse in China», Public Choice 134(1-2): 31-46.

Madden, M. 2012. Privacy Management on Social Media Sites: Most Users Choose Restricted Privacy Settings while Profile 'Pruning' and Unfriending People is on the Rise. Pew Research Center's Internet \& American Life Project.

Madianou, M., Miller, D. 2012. Migration and New Media. London: Routledge.

Malaby, T. 2009. Making Virtual Worlds. Ithaca, NY: Cornell University Press.

Mangold, W.G., Faulds, D.J. 2009. «Social media: The new hybrid element of the promotion mix", Business Horizons 52 (4): 357-365.

Marshall, J. 2002. A Kalahari Family (director of the film).

Marvin, C. 1988. When Old Technologies Were New. New York: Oxford University Press.

Marwick, A., boyd, d. 2014. «Networked privacy: How teenagers negotiate context in social media». New Media \& Society, 16(7): 1051-1067.

Marwick, A. 2011. "I Tweet Honestly, I Tweet Passionately: Twitter Users, Context Collapse, and the Imagined Audience», New Media \& Society 13(1): 114-133.

Maslow, A.H. 1943. "A Theory of Human Motivation», Psychological Review 50(4): 370-396

Mauss, M. 1966. The Gift. London: Cohen and West.

McDonald, T. 2016. Social Media in Rural China. London: UCL Press.

McKay, D. 2007. "Sending Dollars Shows Feeling: Emotions and Economies in Filipino Migration", Mobilities 2(2): 175-194. 
— 2011. "On the Face of Facebook: Historical Images and Personhood in Filipino Social Networking», History and Anthropology 21(4): 483-502.

McLaughlin, C., Vitak, J. 2012. "Norm Evolution and Violation on Facebook", New Media \& Society 14(2): 299-315.

Mehdizadeh, S. 2010. «Self-Presentation 2.0: Narcissism and Self-Esteem on Facebook», Cyberpsychology, Behaviour and Social Networking, 13(4): 357-364.

Mendleson, A., Papacharissi, Z. 2001. «Look at Us: collective narcissism in college student Facebook photo galleries», in Papacharissi, Z., ed. A Networked Self: Identity, community, and culture on social network sites. New York: Routledge, 251-273.

Miller, D., Horst, H. 2006. The Cell Phone. Oxford: Berg.

— 2012 «Introduction», in Horst, H., Miller, D., eds. Digital Anthropology. London: Berg.

Miller, D., Sinanan, J. 2014. Webcam. Cambridge: Polity Press.

— 2017. Visualising Facebook. London: UCL Press.

Miller, D., Slater, D. 2000. The Internet: An Ethnographic Approach. Oxford: Berg.

Miller, D. 1998. A Theory of Shopping. Cambridge: Polity Press.

- 2011. Tales from Facebook. Cambridge: Polity Press.

— 2014. «Hospices: The Potential for New Media», http://www.ucl.ac.uk/ anthropology/

people/academic_staff/d_miller/mil-28.

- 2015a. "Photography in the Age of Snapchat», Anthropology and Photography 1.

- 2015b. "The Tragic Dénouement of English Sociality», Cultural Anthropology 30(2): 336-357.

- 2016. Social Media in an English Village. London: UCL Press.

Morozov, E. 2012. The Net Delusion: The Dark Side of Internet Freedom. New York, NY: Public Affairs.

— «Iran: Downside to the "Twitter Revolution"», Dissent 56(4): 10-14.

Morris, B. 1991. Western Conceptions of the Individual. Oxford: Berg.

Muise, A., Christofides, E., Desmarais, S. 2009. «More information than you ever wanted: does Facebook bring out the green-eyed monster of jealousy?», Cyberpsychological Behavior 12(4): 441-444.

Narayan, G. 2007. "Addressing the Digital Divide: E-governance and M-governance in a Hub and Spoke model», The Electronic Journal on 
Information Systems in Developing Countries 31(1): 1-14.

Naughton, J. 2012. From Guttenberg to Zuckerberg. London: Quercus.

Nicolescu, R. 2016. Social Media in Southeast Italy. London: UCL Press.

Nisbett, N. 2009. Growing Up in the Knowledge Society: Living the IT Dream in Bangalore. New Delhi: Routledge.

Noelle-Neumann, E. 1974. "The spiral of silence: a theory of public opinion", Journal of Communication 24(2): 43-51.

Noor Al-Deen, H., Hendricks, J., eds. 2012. Social Media: Usage and Impact. Lanham, MD: Lexington Books.

Norris, P. 2001. Digital Divide: Civic Engagement, Information Poverty, and the Internet Worldwide. Cambridge: Cambridge University Press.

Nur Muhammad, R., Horst, H.A., Papoutsaki, E., Dodson, G. 2015. «Uyghur transnational identity on Facebook: on the development of a young diaspora", Identities 23(4):485-499.

O’Keeffe, G.S., Clarke-Pearson, K. 2011. "Clinical Report - The Impact of Social Media on Children, Adolescents, and Families», American Academy of Pediatrics,

http://pediatrics.aappublications.org/content/early/2011/03/28/ peds.20110054.full.pdf + html?ijkey=76f29031adb1f95a04cca23436b5 ccdebfd5cd9f.

Oates, S., Owen, D., Gibson, R. K., eds. 2006. The Internet and Politics. Citizens, Voters and Activists. New York: Routledge.

Okabe, D., Ito, M. 2006. «Everyday contexts of camera phone use: Steps toward technosocial ethnographic frmeworks», in Höflich, J.R., Hartmann, M., eds. Mobile Communication in Everyday Life: Ethnographic Views, Observations and Reflections. Berlin: Frank \& Timme, 79-102.

Oosterbaan, M. 2010a. "Virtual Migration. Brazilian Diasporic Media and the Reconfigurations of Place and Space», Revue Européenne des Migrations Internationales 26(1): 81-102.

- 2010b. "Virtual Re-evangelization: Brazilian Churches, Media and the Postsecular City», in Beaumont, J., Molendijk, A., Jedan, C., eds., Exploring the Postsecular: The Religious, The Political, the Urban, Leiden: Brill, 281-308.

Ortiz, S. 1994. "Work, the Division of Labour and Co-operation", in Ingold, T., ed. Companion Encyclopedia of Anthropology. London: Taylor $\&$ Francis.

Ortner, S.B. 1972. «Is Female to Male as Nature Is to Culture?», Feminist Studies 1(2): 5-31. 
Paechter, C. 2013. «Young Women Online: Collaboratively Constructing Identities», Pedagogy, Culture and Society 21(1): 111-127.

Papacharissi, Z. 2002. "The Virtual Sphere: The Internet as a Public Sphere», New Media \& Society 4(1): 9-27.

- 2004. "Democracy Online: Civility, Politeness, and the Democratic Potential of Online Political Discussion Groups», New Media \& Society 6(2): 259-283.

- 2010a. A Private Sphere: Democracy in a Digital Age. Cambridge: Polity Press, 138-144.

- 2010b. "The virtual sphere 2.0: The Internet, the Public Sphere, and Beyond", in Chadwick, A., Howard, P.N., eds. Routledge Handbook of Internet Politics. Oxford: Taylor \& Francis, 230-245.

— ed. 2011. A Networked Self: Identity, Community and Culture on Social Network Sites. London: Routledge.

Parks, M. 2011. "Social network sites as virtual communities», in Papacharissi, Z., ed. A networked self: Identity, community, and culture on social network sites. London: Routledge, 105-123.

Piketty, T. 2014. Capital in the Twenty-First Century. Cambridge: Belknap Press.

Pink, S. 2001. 'Doing Visual Ethnography:' Images, Media and Representation in Research. London: Sage.

- 2011. "Amateur photographic practice, collective representation and the constitution of

Place», Visual Studies 26(2): 92-101.

Pinney, C. 2011. Photography and Anthropology. London: Reaktion.

Plant, S. 1997. Zeros and Ones: Digital Women and the New Technoculture. London: Fourth Estate.

Plato. 2008 (360 BC). Phaedrus. Charleston, SC: Forgotten Books.

Poster, M. 1997. "Cyberdemocracy: Internet and the Public Sphere», Internet Culture: 201-218.

Postill, J. 2008. "Localizing the Internet Beyond Communities and Networks", New Media \& Society 10(3): 413-431.

— 2012. "Digital Politics and Political Engagement». in Horst, H., Miller, D., eds. Digital Anthropology. London: Berg.

Potter, J. 2011. «New Literacies, New Practices and Learner Research: Across the Semi-Permeable Membrane between Home and School», Lifelong Learning in Europe (3): 174-180. 
Prensky, M. 2001. «Digital Natives, Digital Immigrants», On the Horizon 9(5): 1-6.

Putnam, R. 2000. Bowling Alone: The Collapse and Revival of American Community. New York-London: Simon \& Schuster.

Qiu, J.L. 2009. Working-Class Network Society: Communication Technology and the Information Have Less in Urban China. Cambridge, MA: The MIT Press.

Qiu, L., Lin, H., Leung, A. K.-y. 2013. «Cultural Differences and Switching of In-Group Sharing Behavior Between an American (Facebook) and a Chinese (Renren) Social Networking Site», Journal of Cross-Cultural Psychology, 44(1): 106-121.

Rainey, L.D. 2010. Confucius \& Confucianism: The Essentials. Malden, MA: Oxford: Wiley-Blackwell.

Rainie, L., Wellman, B. 2012. Networked. Cambridge, MA: The MIT Press. Rangaswamy, N., Arora, P. 2015. "The Mobile Internet in the Wild and Every Day: Digital Leisure in the Slums of Urban India», International Journal of Cultural Studies, 19(6): 611-626.

Rantanen, T. 2005. The Media and Globalization. New York: Sage.

Rheingold, H. 1993. Virtual Community: Homesteading on the Electronic Frontier. New York: Addison Wesley.

- 2012. Net Smart: How to Thrive Online. Cambridge, MA: The MIT Press.

Ritchie, D. 2005. "Frame-Shifting in Humor and Irony», Metaphor and Symbol 20(4): 275-294.

Rousseau, Jean-Jacques. 2010 (1754). Discourse on the Origin and Foundations of Inequality Among Men. Rosenblatt, H., trans and ed. Boston, MA: Bedford/St. Martin's.

Rubel, A.J. 1977. "Limited Good' and 'Social Comparison': Two Theories, One Problem», Ethos 5(2): 224-238.

Sagioglou, C., Greitemeyer, T. 2014. «Facebook’s Emotional Consequences: Why Facebook Causes a Decrease in Mood and Why People Still Use It», Computers in Human Behavior 35: 359-363.

Sahlins, M. 1972. Stone Age Economics. Chicago, IL: Aldine-Atherton.

Salvatore, A., ed. 2011. "Between Everyday Life and Political Revolution: the Social Web in the Middle East», Oriente Moderno, n.s. XCI/1.

Schrooten, M. 2012. «Moving ethnography online: Researching Brazilian migrants' online togetherness", Ethnic and Racial Studies 35(10): 1794-1809. 
Scobie, W. 2011. "An Anthropological Introduction to YouTube by Michael Wesch", American Anthropologist 113(4): 661-662.

Seib, P. 2012. Real-time diplomacy: Politics and power in the social media era. Basingstoke: Palgrave Macmillan.

Sen, A. 1992. Inequality Re-examined. Cambridge, MA: Harvard University Press.

- 1999. Development as Freedom. Oxford: Oxford University Press.

Sennett, R. 1977. The Fall of Public Man. New York: Knopf.

Shade, L. R. 2002. Gender and Community in the Social Construction of the Internet. New York: Peter Lang.

Shirky, C. 2008. Here Comes Everybody: The Power of Organizing without Organizations. New York: Penguin.

Simmel, G., Wolff, Kurt H. 1950. The Sociology of Georg Simmel. Glencoe, IL: Free Press.

Simmel, G. 1968. The Conflict in Modern Culture and other Essays. New York: Teachers College Press.

Sinanan, J. 2017. Social Media in Trinidad. London: UCL Press.

Skeggs, B. 1997. Formations of Class and Gender: Becoming Respectable. London: Sage.

Slater, D. 2014. New Media, Development and Globalization: Making Connections in the Global South. Cambridge: Polity Press.

Smith, P.K., Brain, P. 2000. «Bullying in Schools: Lessons from Two Decades of Research", Aggressive Behavior 26(1): 1-9.

Sprague, S. 1978. "How I See the Yoruba See Themselves», Studies in the Anthropology of Visual Communications. 5(1): 9-29.

Spyer, J. 2017. Social Media in Emergent Brazil. London: UCL Press.

Stafford, C. 1995. The Roads of Chinese Childhood: Learning and Identification in Angang. Cambridge: Cambridge University Press.

Street, B. 2003. "What's "New" in New Literacy Studies? Critical Approaches to Literacy in Theory and Practice», Current Issues in Comparative Education 5(2): 77-91.

Tacchi, J. 2012. «Digital Engagement», in Horst, H., Miller, D., eds. Digital Anthropology. London: Berg. 225-241.

Tandoc, E.C. Jr., Ferruci, P., Du y, M. 2015. «Facebook Use, Envy, and Depression Among College Students: Is Facebooking Depressing?», Computers in Human Behavior 43: 139-146.

The 34th Statistical Report on Internet Development in China. Scaricato il 28 
marzo 2015. http:// www1.cnnic.cn/IDR/ReportDownloads/201411/ P020141102574314897888.pdf

The Economist, 28 February 2015.

The Economist, 13 September 2014.

Thin, N. 2005. "Happiness and the Sad Topics of Anthropology», WeD Working Paper 10. ESRC Research Group on Wellbeing in Developing Countries.

Thompson, C. 2013. Smarter than you think. London: Penguin.

Tiggeman, M., Slater, A., 2013. «NetGirls: The Internet, Facebook, and Body Image Concern in Adolescent Girls", International Journal of Eating Disorders 46(6): 630-633.

Trepte, S., Reinecke, L., eds. 2011. Privacy Online: Perspectives on Privacy and Self-Disclosure in the Social Web. New York: Springer.

Tsui, L. 2015. «The coming colonization of Hong Kong cyberspace: government responses to the use of new technologies by the umbrella movement", Chinese Journal of Communication. 8(4): 1-9.

Tufekci, Z., Wilson, C. 2012. "Social media and the decision to participate in political protest: Observation from Tahrir Square», Journal of Communication 62(2): 363-379.

Tufekci, Z. 1997. Life on the screen: Identity in the Age of the Internet. New York: Simon and Schuster.

— 2014. "The Medium and the Movement: Digital Tools, Social Movement Politics, and the End of the Free Rider Problem», Policy \& Internet 6 (2): 202-208.

Turkle, S. 2011. Alone Together: Why We Expect More from Technology and Less from Each Other. New York: Basic Books.

Uimonen, P. 2012. Digital Drama. New York: Routledge.

Utz, S., Kramer, N. 2009. "The privacy paradox on social network sites revisited: The role of individual characteristics and group norms», Cyberpsychology: Journal of Psychosocial Research on Cyberspace 3(2).

Valenzuela, S., Park, N., Kee, K. F. 2009. «Is There Social Capital in a Social Network Site?: Facebook Use and College Students' Life Satisfaction, Trust and Participation», Journal of Computer-Mediated Communication 14(4): 875-901.

Van Dijck, J. 2007. Mediated Memories in the Digital Age. Stanford, CA: Stanford University Press.

- 2008. "Digital photography: Communication, identity, memory», 
Visual Communication 7: 57-76.

- 2013a. The Culture of Connectivity. Oxford: Oxford University Press.

- 2013b. "You have one identity": performing the self on Facebook and LinkedIn", Media, Culture \& Society 35(2): 199-215.

Van Dijk, J.A.G.M. 1999. The Network Society: Social Aspects of New Media. London: Sage.

— 2013. "Inequalities in the Network Society», in Orton-Johnson, K., Prior, N., eds. Digital Sociology. London: Palgrave Macmillan.

Van Doorn, N., Van Zoonen, L., Wyatt, S. 2007. «Writing from Experience:

Presentations of Gender Identity on Weblogs», European Journal of Women's Studies 14(2): 143-159.

Van House, N.A., Davis, M. 2005. "The Social Life of Camera Phone Images", Proceedings of the Pervasive Image Capture and Sharing: New Social Practices and Implications for Technology Workshop (PICS 2005) at the Seventh International Conference on Ubiquitous Computing (UbiComp 2005).

Venkatraman, S. 2017. Social Media in South India. London: UCL Press.

Vidyarthi, L.P., ed. 1984. Applied anthropology in India: principles, problems, and case studies. New Delhi: Kitab Mahal.

Voida, A., Mynatt, E.D. 2005. "Six Themes of the Communicative Appropriation of Photographic Images", Proceedings of the SIGCHI Conference on Human Factors in Computing Systems. 171-180.

Wajcman, J. 2004. TechnoFeminism. Cambridge: Polity Press.

Wali, A. 2012. "A Different Measure of Well-Being» Vital Topics Forum. Johnston, B.R., ed. American Anthropologist 114(1): 12.

Wallis, C. 2011. «New Media Practices in China: Youth Patterns, Processes, and Politics», International Journal of Communication 5: 406-436.

- 2013. Technomobility in China: young migrant women and mobile phones. New York, London: New York University Press.

Wallman, S., ed. 1979. Social Anthropology of Work. Vol. 19. London: Academic Press.

Wang, R.R. 2002. "Globalizing the Heart of the Dragon: The Impact of Technology on Confucian Ethical Values", Journal of Chinese Philosophy 29(4): 553-569.

Wang, X. 2016. Social Media in Industrial China. London: UCL Press.

Warner, M. 2002. "Publics and counterpublics», Public culture 14(1): 49-90. 
Warschauer, M. 2004. Technology and Social Inclusion: Rethinking the Digital Divide. Cambridge, MA: The MIT Press.

Weber, M. 2002 (1905). The Protestant Ethic and the Spirit of Capitalism. London: Penguin Books.

Weller, K. et al., eds. 2013. Twitter and Society. New York: Peter Lang.

Wenger, E. 1998. Communities of practice: learning, meaning, and identity. Cambridge: Cambridge University Press.

Wesch. M. 2008. 'An Anthropological Introduction to YouTube.' YouTube, 26 July 2008. http:// www.youtube.com/ watch?v=TPAO- 1Z4_hU, (accesso 14 settembre 2014).

Wilson, R., Gosling, S., Graham, L. 2012. «A Review of Facebook Research in the Social Sciences", Perspectives on Psychological Science 7(3) 203-220. Yan, Y. 1996. The Flow of Gifts: Reciprocity and Social Networks in a Chinese Village. Stanford, CA: Stanford University Press.

Zelizer, V. 2011. Economic Lives. Princeton, NJ: Princeton University Press. Zickuhr, K. 2013. Who's Not Online and Why. Washington DC: Pew Research Center's Internet \& American Life Project. 


\section{Sitografia}

http://blogs.ucl.ac.uk/global-social-media/2013/11/24/what-willwe-learn-from-the-fall-of-facebook/.

http://blogs.ucl.ac.uk/global-social-media/2014/08/31/the-qualitativeinsights-we-get-from-applying-questionnaires/.

http://time.com/selfies-cities-world-rankings (accesso 20 luglio 2015).

http://www.al-monitor.com/pulse/originals/2013/09/turkeys-akp-twitter-election.html.

http://www.dailymail.co.uk/news/article-2419419/All-lonely-Facebookfriends-Study-shows-

social-media-makes-MORE-lonely-unhappy-LESS-sociable.html.

http://www.danah.org/researchBibs/sns.php.

http://www.hurriyetdailynews.com/ruling-akp-hires-thousands-for-newsocial-media-campaign.aspx?pageID =238\&nID =54479\& 2 NewsCatID = 338.

http://www.idc.com/prodserv/smartphone-market-share.jsp (accesso 26 agosto 2015).

http://www.media-anthropology.net/.

http://www.newyorker.com/tech/elements/how-facebook-makesus-unhappy.

http://www.nytimes.com/2008/07/26/business/worldbusiness/26internet. html?_r=0.

http://www.theatlantic.com/technology/archive/2014/05/qr-codes-forthe-dead/370901/.

http://www.theguardian.com/commentisfree/2014/sep/11/when-takingselfies-in-trinidad-its-whats-on-the-outside-that-matters.

http://www.theguardian.com/media-network/media-network-blog/2014/ mar/13/selfie-social-media-love-digital-narcassism.

http://www.theguardian.com/technology/2013/dec/06/selfies-statusupdates-digital-bragging-web.

http://www.theguardian.com/media-network/media-network-blog/2014/ mar/13/selfie-social-media-love-digital-narcassism.

http://www.theguardian.com/world/2014/jun/05/internet-use-mobile- 
Come il mondo ha cambiato i social media

phones-africa-predicted-increase-20-fold.

http://www.wantchinatimes.com/news-subclass-cnt.aspx? id=2014 $0205000127 \&$ cid $=1102$.

http://www.whywepost.com.

http://www.wsj.com/articles/SB10001424127887323527004579079151 479634742.

http://www.youtube.com/whywepost

https://press.linkedin.com/about-linkedin (accesso 28 agosto 2015). 


\section{Sintesi dei contenuti}

\section{Capitolo 1}

\section{Che cosa sono i social media?}

Sarebbe auspicabile considerare i social media non tanto come le piattaforme su cui le persone postano, piuttosto come i contenuti che sono postati su queste piattaforme. Questi contenuti variano moltissimo da area a area, ragion per cui serve uno studio comparativo. Il modo in cui in una certa località descriviamo i social media dunque non andrebbe inteso come una descrizione generale dei social media quanto, piuttosto, come un caso locale.

Quello dei social media è oggi l'ambito entro cui socializziamo, non si tratta di un semplice strumento di comunicazione. Prima dei social media c'erano per lo più media per le conversazioni private o per la trasmissione pubblica di programmi.

Avanziamo qui una teoria della "socialità modulabile", per mostrare come i social media abbiano colonizzato la spazio della socialità di gruppo compreso tra il privato e il pubblico. Nel fare ciò hanno creato delle scale, che includono la misura del gruppo e il grado di privacy.

Adottiamo poi una teoria polimediale che pone in risalto la nostra incapacità di capire qualsivoglia piattaforma o media se considerati separatamente. Essi vanno visti in relazione reciproca, dato che le persone usano l'intera gamma di possibilità che hanno a disposizione per scegliere specifiche piattaforme o specifici media in relazione a specifici generi di interazione.

Rifiutiamo una nozione di 'virtuale' che porta a separare lo spazio dell'online come un mondo a se stante. Consideriamo i social media come parte integrante della vita quotidiana proprio come le conversazioni telefoniche che sono oggi a tutti gli effetti parte della vita offline e non di un ambito separato.

Proponiamo una teoria dell'acquisizione che si oppone all'idea che con le nuove tecnologie digitali avremmo perso alcuni elementi essenziali del nostro essere umani o saremmo diventati post-umani. 


\section{Capitolo 2}

\section{Gli studi sui social media}

Accettiamo ovviamente che la nostra definizione e il nostro approccio siano solo uno fra molti possibili, e che ogni disciplina dalla prospettiva che le è propria offra un contributo utile a capire la natura dei social media.

Le piattaforme dei social media come Orkut e MySpace vengono di frequente sostituite, altre, per esempio Facebook, sono in continua trasformazione. Ne consegue che anche le nostre definizioni e i nostri approcci devono essere dinamici.

Nel determinare i contenuti che ospitano (vale a dire il perché le persone postano specifici tipi di contenuti proprio su quella piattaforma), le piattaforme e le loro proprietà sono molto meno importanti di quanto possiamo presumere. Tipi di contenuti, per esempio le battute ironiche degli studenti, migrano con estrema leggerezza da una piattaforma a un'altra interamente diversa e con caratteristiche molto molto differenti.

Rifiutiamo l'idea che lo sviluppo di Internet sia rappresentabile come un unico percorso. Alcuni degli aspetti più rilevanti dei social media sembrano esattamente l'opposto rispetto a precedenti utilizzi di internet. Per esempio, quello che in internet è il problema dell'anonimato, nei social media diventa un problema di perdita di privacy.

\section{Capitolo 3 \\ Metodo e approccio}

La nostra formazione antropologica è dimostrata dai 15 mesi di ricerca etnografica in ciascuna delle nove comunità, e dalla nostra intenzione di concentrare interessi iniziali e focus preferenziale su ciò che è risultato avere maggiore importanza per ciascuna comunità.

Abbiamo ritenuto fossero necessari 15 mesi per occuparci di tutte le diverse categorie di persone presenti in ogni luogo - anziani e giovani, più e meno istruiti, di differente condizione economica, ecc. - e ottenere il grado di fiducia richiesto per entrare a far parte degli ambiti più privati, come per esempio WhatsApp.

L'etnografia riflette la realtà, che nessuno vive in un solo contesto. Ogni nostra azione e interazione sono collegate in quanto parte della nostra 
vita, per questo il nostro approccio all'esperienza delle persone deve essere olistico.

Il metodo principale dell'antropologia è l'empatia: il tentativo di comprendere i social media dal punto di vista dei loro utenti.

A differenza di gran parte dell'antropologia classica, questo progetto è stato sempre all'insegna della collaborazione e della comparazione, dalla sua concezione, alla realizzazione fino alla disseminazione dei risultati.

\section{Capitolo 4}

\section{I risultati della nostra indagine}

Presentiamo i risultati di un questionario somministrato a 1119 informatori tra i nostri nove siti. Le tematiche indagate sono 26, e vanno dal se - e con chi - si condividono le password e le differenti categorie di followers fino a se gli utenti rispondono agli annunci pubblicitari o se i social media incrementano la loro attività politica.

Come indicazione generale precisiamo che i risultati vanno presi con molta cautela, dato che spesso il modo più plausibile di spiegare le risposte emerse dall'indagine è che le persone hanno interpretato le domande in modi differenti e culturalmente specifici.

Gli esiti di tali indagini quantitative a carattere comparativo possono quindi essere interpretati in modo appropriato solo grazie alle ulteriori conoscenze culturali che derivano dal lavoro etnografico qualitativo.

\section{Capitolo 5}

\section{Istruzione e giovani}

C'è notevole preoccupazione nell'opinione pubblica sul fatto che i social media distraggano dallo studio e contraggano le abilità sociali dei giovani - a dispetto di una significativa mole di ricerca preesistente che mette in guardia dal trarre facili conclusioni di questo genere.

In diversi dei nostri campi di ricerca mettiamo in luce che le famiglie a basso reddito spesso vedono l'attività sui social come un'abilità molto utile, che rinforza la capacità di leggere e scrivere e apre una strada verso canali formativi alternativi e informali. Al contrario, le famiglie a più alto reddito 
li vedono maggiormente come una minaccia contro l'istruzione formale. In ogni caso, abbiamo anche fatto ricerca in campi dove potrebbe essere vero il contrario.

Questo punto illustra i rischi di generalizzare con riferimento alla Cina, poiché i due siti cinesi discussi in questo capitolo mostrano l'uno il più alto grado di fiducia nell'istruzione formale e l'altro il più basso.

Il modo migliore di cogliere l'impatto dei social media è focalizzarsi su specifici gruppi di relazioni: quelle fra studenti, quelle fra insegnanti e studenti e fra entrambe le parti di questi gruppi e i familiari. Li analizziamo uno alla volta.

\section{Capitolo 6}

\section{Attività lavorativa e commerciale}

In questo caso le preoccupazioni principali amplificate dalla stampa sono il controllo da parte delle aziende, nuove forme di mercificazione e i social media come intrattenimento.

Anche se le piattaforme dei social sono proprietà di aziende private, i social media non necessariamente favoriscono gli interessi della vendita. Per esempio, sulla scia di ciò che li ha preceduti, come l'email, si rivelano strumenti potentissimi con cui il pubblico ha nettamente respinto i tentativi del mercato di separare il mondo del lavoro da quello della famiglia. Nel caso dell'India del Sud i social media aiutano a tenere il lavoro all'interno della famiglia.

Nella maggior parte dei nostri campi di ricerca le persone erano di gran lunga più preoccupate del possibile controllo da parte di altre persone, magari conosciute, che da parte delle loro aziende.

Tuttavia, nel sito dove si è svolta la ricerca in Turchia sudorientale ci si preoccupa del controllo da parte dello Stato, mentre in quello inglese l'aumento della pubblicità mirata ha rivelato un livello di conoscenza da parte delle aziende che ha ricadute negative sulle aziende dei social media.

I social in gran parte dei nostri campi di ricerca risultavano più importanti nel sostenere attività imprenditoriali di piccola scala che fanno leva sulle connessioni personali delle persone (come bar e locali a Trinidad o vendita di vestiti usati in Cile), piuttosto che nell'agire a favore della vendita su grande scala. 
Questo punto illustra in modo chiaro le differenze nel modo in cui in società diverse si considera il denaro come parte integrante delle relazioni personali oppure come del tutto esterno a esse. Ciò si riflette nel contrasto fra un sito come Amazon, che tende a essere impersonale, e l'equivalente cinese Taobao, che mantiene il carattere personale della comunicazione all'interno della transazione commerciale.

\section{Capitolo 7 \\ Relazioni online e offline}

In questo caso la preoccupazione principale è se relazioni superficiali, inautentiche, come quelle online, stiano sostituendo le più profonde relazioni offline. In molti casi dimostriamo che le interazioni online sono nei fatti un altro lato delle medesime relazioni offline. Invece che aumentare la mediazione, i social media aiutano a scoprire la natura primariamente mediata della comunicazione e della socialità, inclusa la comunicazione cosiddetta "faccia a faccia".

Nel nostro campo di ricerca nella Cina industriale abbiamo percepito che, per spingere le persone più vicino alla vita moderna, alla quale aspirano, ha forse fatto di più il passaggio dall'offline all'online che lo spostamento dai villaggi all'industria.

In alcune società, come a Trinidad, l'aumento della visibilità delle persone attraverso i social media porta gli utenti a vedere queste rappresentazioni come potenzialmente più verosimili delle osservazioni offline di coloro che li circondano.

In alcune società, come nei nostri campi di ricerca brasiliani e a Trinidad, i social media alimentano una tendenza a chiedere l'amicizia agli amici dei propri amici o familiari. In altre, per esempio nel sito della Cina rurale, i social media alimentano relazioni totalmente nuove, comprese le richieste di amicizia rivolte a sconosciuti.

L'uso dei social potrebbe risultare complementare alla socializzazione, piuttosto che rifletterne altre forme. Per esempio, nel nostro sito di ricerca nell'Italia meridionale le persone sentivano di avere già sufficienti impegni sociali, e per questo facevano un uso minore di social media. 


\section{Capitolo 8}

\section{Genere}

Il sito della nostra ricerca nella Turchia sudorientale è uno dei tanti a suggerire che i social media visibili al pubblico, come Facebook, possono far aumentare l'apparenza di conservatorismo o diventare un luogo ultraconservatore. I cambiamenti nella vita offline non sono rappresentati in questo spazio pubblico soggetto alla sorveglianza dei familiari. Rappresentazioni di genere in senso conservatore sono alimentate anche nel sud dell'India, nella Cina rurale e nei nostri siti in Cile.

$\mathrm{Al}$ contempo, i social media più privati, come WhatsApp, hanno avuto un effetto liberatorio sulle vite delle giovani donne nello stesso sito della Turchia sudorientale musulmana, creando possibilità inattese di contatto tra i generi e l'appagamento di aspirazioni romantiche.

Nel nostro terreno di ricerca nel sud Italia le donne rinnegano le forme in cui hanno postato prima di sposarsi, per apparire come mogli e madri. A Trinidad, tuttavia, le donne tendono a enfatizzare la loro capacità di sex appeal nonostante il matrimonio e la maternità.

I social media potenziano la capacità di vedere in che modo le differenze e gli stereotipi di genere sono messi a fuoco e rappresentati - non di rado per mezzo di costanti associazioni del tipo 'birra' (maschile) e 'vino' (femminile) emerse nel nostro campo di ricerca inglese o del tipo 'lavoro manuale' (per gli uomini) e 'lavori di cura' (per le donne) emerse nel nostro sito cileno.

Nel campo brasiliano è attestabile in una certa misura un aumento dell'uguaglianza di genere e in diversi siti si osserva la crescente visibilità on line di forme non normative di sessualità.

\section{Capitolo 9 Diseguaglianza}

La comparazione evidenzia come sia importante riconoscere che se, da un lato, social media e smartphone possono creare un più alto grado di uguaglianza nella capacità di comunicare e di socializzare all'interno di società fortemente diseguali, nello stesso tempo questo può non avere alcun impatto sulla diseguaglianza offline, fuori dalla rete.

Il modo in cui i desideri delle persone sono rappresentati on line è altamente 
variabile. Gli operai cinesi fantasticano sulla loro vita futura mentre i brasiliani evangelici si focalizzano sulle prove dei loro progressi in fatto di rispettabilità.

Le persone usano i social sia per screditare in modo umoristico o ironico proclami pretenziosi in fatto di benessere e istruzione, sia anche per ostentare tali proclami. Nel nostro sito cileno i social media sono usati per cancellare le differenze di reddito, insieme ad altre rivendicazioni di un'identità separata come l'appartenenza indigena e l'etnicità, al fine di esprimere una solidarietà comune in opposizione alle aree metropolitane che i locali avvertono come superficiali e da cui si sentono sfruttati economicamente.

Nell'India meridionale si può notare che i social media hanno aggiunto una nuova dimensione della differenza sociale - il relativo cosmopolitismo che si rivela postando - alle tradizionali forme di diseguaglianza di casta e di classe.

\section{Capitolo 10 Politica}

Molti studi precedenti esagerano l'impatto dei social media sulla politica focalizzandosi sugli usi più facilmente osservabili, le discussioni o l'attivismo visibili su Twitter. Di contro, il nostro studio si limita a osservare in che misura i post di contenuto politico appaiono nell'uso quotidiano dei social da parte delle persone.

Il nostro campo di ricerca nella regione curda della Turchia mostra perché sembra che la politica compaia meno sui social laddove c'è tensione e rischio. Qui molti post, come nel sud dell'India, sono prudenti e convenzionali. La preoccupazione principale riguarda il potenziale impatto del post sulle proprie relazioni sociali.

In molti siti, per esempio in Inghilterra e a Trinidad, i social media sfruttano l'ambito della politica soprattutto con finalità di intrattenimento.

Se da un lato si ha un uso limitato dei social per commentare questioni politiche locali, dall'altro si usano per creare solidarietà locale grazie a post negativi su questioni nazionali, come per la corruzione in Italia o in Cina. In Cina raramente la censura scende sino a questo tipo di comunità, e sono le compagnie di social media che controllano la disseminazione delle notizie. In Turchia sudorientale, tuttavia, le persone che postano opinioni antigovernative corrono un rischio personale. 


\section{Capitolo 11}

\section{Immagini visive}

Un effetto rilevante dei social media è che la comunicazione umana è diventata più visiva a danno di quella orale e testuale.

I meme sono particolarmente significativi di un certo tipo di ordine morale in internet. Facendone uso, le persone sono in grado di esprimere i propri valori e screditare quelli altrui in modi meno diretti e più accettabili che in passato.

Le generalizzazioni riguardanti nuove forme visuali, come i selfie, spesso non sono accurate. Ci sono diverse varietà di selfie spesso usate per esprimere senso di appartenenza piuttosto che narcisismo individuale.

L'aumento della visibilità spesso è associato con l'aumento del conformismo, e in alcuni casi, come nella Turchia sudorientale, si preferiscono argomenti sicuri come il cibo rispetto a fotografie di persone che potrebbero dar luogo a pettegolezzi. Di contro, a Trinidad gossip e 'stalking' potenziati sono un piacere favorito dai social media molto ben accetto. Il caso di Trinidad è un campanello di allarme sul ruolo delle differenze culturali nei modi in cui le persone associano materiali visivi e verità.

Saper comunicare soprattutto mediante forme visuali è importante specialmente per coloro che hanno problemi con la capacità di leggere e scrivere. Esempi in questo senso sono brasiliani anziani a basso reddito, utenti più giovani che scelgono piattaforme tipo Instagram e Snapchat e gruppi con relazioni sociali precarie, per esempio gli operai cinesi.

\section{Capitolo 12 Individualismo}

Esiste il timore che i social media, insieme a quasi ogni altro tipo di innovazione tecnologica, alimentino l'individualismo a scapito della socialità. Ciò che abbiamo trovato, tuttavia, dimostra che mentre le prime forme di internet favorivano un lavoro di rete ego-centrato, i social media, in molti siti, rappresentano un parziale ritorno al precedente gruppo di socializzazione, come la famiglia. Questo può includere le tradizioni 'confuciane' del rispetto familiare nella Cina rurale, ma anche comprendere la casta in India o la tribù per la comunità curda in Turchia.

In molti contesti tradizionali, tuttavia, ci sono anche rinnovate opportunità 
di networking individualizzato: i nostri dati dimostrano che i social media possono dar corpo simultaneamente a due tendenze opposte, come del resto spesso accade. La socialità modulabile può alimentare sia gruppi tradizionali sia, come nel caso di WhatsApp, gruppi di piccola scala spesso temporanei.

I social media non sono semplicemente una cornice per fare amicizia. Come nel nostro "Goldilocks Strategy" fondato in Inghilterra, sono spesso usati come un modo per tenere le persone alla giusta distanza. In altri siti la sociabilità modulabile è utilizzata per differenziare le piattaforme in luoghi per la socializzazione più privati o più pubblici.

Il dibattito pubblico sulla privacy e sui social media emerge come molto provinciale. Considerati in molti paesi come una nuova minaccia alla privacy, in altri, come nel sud dell'India e in Cina, i social media offrono ad alcune persone la prima occasione di sperimentare un autentico spazio privato.

Persino dove i social sono usati per esprimere una dimensione individuale, la amplificazione della visibilità tende a renderla sempre più conformista rispetto agli stili di individualismo culturalmente accettati.

\section{Capitolo 13}

\section{I social media rendono le persone più felici?}

Lo studio dei social media può aiutarci nella critica di qualsiasi idea semplice o eccessivamente generica di cosa significhi essere felici o dichiarare di esserlo. Anche se si tratta di concezioni locali di felicità, nella maggior parte dei casi sono scarse le prove a supporto delle tesi giornalistiche che in generale i social media hanno reso le persone meno felici o soddisfatte. Ancora, si esprime la preoccupazione che i social media, nella loro componente ludica, incoraggino il perseguimento di piaceri transitori, o che, con la diffusione dell'acquisto di abiti online, accrescano lo stress legato a come ci presentiamo in pubblico.

I social media hanno quanto meno aumentato la spinta ad apparire felici online. Possono però anche essere il luogo in cui le persone sono in grado di articolare visivamente le loro aspirazioni a una vita felice, come è il caso di una classe sociale emergente nel nostro campo di ricerca brasiliano o di una rinnovata rispettabilità domestica in Cile.

Di contro, in altri siti i social media possono esprimere adesione e soddisfazione rispetto ai valori tradizionali. Questi valori possono includere quelli 
familiari nella Cina rurale, ideali di bellezza in Italia, di comunità a Trinidad, dell'Islam nella Turchia sudorientale o di stretta parentela nell'India del sud.

\section{Capitolo 14 Il futuro}

Questa capitolo inizia con il riconoscimento della inseparabilità dei social media dal nuovo carattere ubiquo degli smartphone in quanto parte della vita quotidiana. È probabile che questa tendenza continui, soprattutto per quanto riguarda le popolazioni a basso reddito e la generazione più anziana, in passato meno presenti sui social.

E possibile che, stante l'invenzione continua di nuove piattaforme che occupano gli interstizi tra il privato e il pubblico, arriveremo ad accettare i media in generale come costitutivi di una scala di socialità senza dover designare un gruppo a parte di piattaforme qualificate come 'social media'. In ogni caso, queste piattaforme potrebbero dissolversi all'interno di un più ampio spettro di applicazioni per il telefono.

In generale, il nostro lavoro ha suggerito che più una società è conservatrice più è grande l'impatto dei social media, anche se l'effetto può essere quello di dare rinforzo a conformismi e conservatorismi, ma anche di creare opportunità inedite di libertà.

Come la maggior parte delle tecnologie digitali, di norma i social media rinforzano contemporaneamente tendenze opposte. Esempi di ciò sono l'incremento di demercificazione e mercificazione, di libertà e di oppressione politica, di localismo e globalismo. E ancora, insieme a altre tecnologie digitali, i social media possono in sé essere usati per presentare il futuro, benché questo ruolo sia destinato a declinare nel tempo.

\section{Conclusioni}

In futuro, proprio come in gran parte oggi, per avere risposte su ciò che $\mathrm{i}$ social media sono, avremo bisogno di ricerche antropologiche sul campo, qualitative e comparative, empaticamente impegnate sui social dal punto di vista dei loro utenti, perché il mondo continuerà a cambiarli. 


\section{Elenco delle immagini}

Fig. 1.1 Socialità modulabile

Fig. 1.2 Presenza sulle piattaforme di social media di ragazzi tra gli 11 e i 18 anni nelle scuole inglesi

Fig. 1.3 Le scale di social media usate dagli scolari inglesi

Fig. 3.1 Venkatraman vestito in modo inappropriato

Fig. 3.2 Sinanan senza velo

Fig. 3.3 Costa con il velo

Fig. 4.1 Numero medio di amici su social media primari - QQ in Cina, Facebook in tutti gli altri siti

Fig. 4.2 Pensi di ... conoscere più persone grazie ai social media? Distribuzione delle risposte attraverso tutti i siti alla domanda se gli utenti ritenessero di conoscere più persone grazie ai social media

Fig. 4.3 Pensi che ... gli individui con più amici offline abbiano anche più amici online? Distribuzione delle risposte alla domanda se gli utenti ritenessero che avere molti amici offline avesse come risultato averne molti on line

Fig. 4.5 Hai mai tolto l'amicizia a qualcuno sui social media per aver postato post politici? Distribuzione delle risposte alla domanda se l'utente avesse mai tolto l'amicizia a qualcuno per post politici

Fig. 4.6 Sei mai uscito con qualcuno che hai incontrato sui social media? Distribuzione di appuntamenti presi

Fig. 4.7 Che percentuale di foto che fai finisce postata sui tuoi profili sui social media? Distribuzione della percentuale di foto postate sui social media

Fig. 4.8 Giochi sui social media? Distribuzione di gioco on line sui social

Fig. 4.9 Distribuzione dell'uso di smartphone per multimedia e intrattenimento nella Cina industriale. Musica - Gioco - Foto - Videoriprese - Video - Radio

Fig. 4.10 Percentuale di persone che condividono la loro password di accesso ai social con famiglia / amici

Fig. 4.11 Meme dal Cile del Nord che esibisce la privacy di un partner sui 


\section{Come il mondo ha cambiato i social media}

social media

Fig. 4.12 Distribuzione di profili social senza nome / foto

Fig. 4.13 Ragioni per account QQ multipli nella Cina industriale. Giochi on line - Sicurezza - Social network diversi.

Fig. 4.14 Ti preoccupa che le persone possano mettere foto tue sui social media? Distribuzione delle risposte alla domanda se gli utenti fossero preoccupati che le persone mettessero sui social loro foto

Fig. 4.15 Hai mai cliccato su una ... pubblicità sui social media? Distribuzione delle risposte alla domanda se gli utenti avessero mai cliccato su pubblicità sui social media.

Fig. 4.16 Hai mai comprato qualcosa perché l'hai vista a un tuo amico sui social media? Distribuzione del comportamento riguardo agli acquisti.

Fig. 4.17 Hai mai guadagnato soldi usando i social media? Distribuzione delle risposte alla domanda se gli utenti avessero mai guadagnato soldi attraverso i social media.

Fig. 4.18 Hai speso denaro reale sui social media o nei giochi on line? Distribuzione di somme spese sui social media o nei giochi on line.

Fig. 4.19 Distribuzione di affari "Liked"/"Followed" sui Social Media.

Fig. 4.20 A che età ritieni accettabile che un bambino cominci a usare i social media? Distribuzione delle risposte alla domanda sull'età ritenuta appropriata.

per un bambino all'uso dei social media

Fig. 4.21 Il tuo posto di lavoro/la tua scuola ti consente di accedere ai tuoi social media durante le ore di impegno? Distribuzione dell'accesso ai social media durante l'impegno lavorativo (posto di lavoro/scuola).

Fig. 4.22 I social media sono buoni o cattivi per l'istruzione? Distribuzione delle risposte alla domanda se i social media abbiano effetti buoni o cattivi sull'educazione.

Fig. 4.23 I social media sono buoni o cattivi per il lavoro? Distribuzione delle risposte alla domanda se i social media abbiano effetti buoni o cattivi sul lavoro.

Fig. 4.24 Ritieni che l'interazione sui social media sia un peso? Distribuzione delle risposte alla domanda se gli utenti ritengano un peso l'interazione sui social media.

Fig. 4.25 Ritieni che i social media ti abbiano reso politicamente più attivo? Distribuzione delle risposte alla domanda se i social media abbiano reso gli utenti politicamente più attivi. 


\section{Elenco delle immagini}

Fig. 4.26 L'uso dei social media ti rende felice? Distribuzione delle risposte alla domanda se l'uso dei social media renda gli utenti più felici.

Fig. 4.27 Distribuzione delle risposte in base al genere

Fig. 11.1 Immagini di fantasie di consumo postate su QQ.

Fig. 11.2 Fig. 11.2 Immagini di bambini scattate in uno studio professionale e postate dai genitori su QQ.

Fig. 11.3 Selfie postati su Facebook da una giovane donna di Trinidad

Fig. 11.4 "Footies" postati da giovani cileni

Fig. 11.5 Biglietti di auguri condivisi dagli abitanti di Trinidad su Facebook.

Fig. 11.6 Saluti pomeridiani e serali circolati su Facebook nel Tamil Nadu.

Fig. 11.7 Immagini di cibo scattate in occasione di riunioni familiari nel sudest della Turchia.

Fig. 11.8 Memi circolati su Facebook nel nordest del Brasile: "Sono andato in chiesa tante volte così... poi Dio mi ha parlato e ora mi sento così!" e "Se avessi voluto far contenti tutti non avrei fatto un profilo su Facebook, avrei fatto un barbeque".

Fig. 11.9 Questo post su QQ recita: "Lascerò le mie lacrime a coloro che mi hanno davvero amato, e lascerò un sorriso a coloro che in passato mi hanno ferito". La persone che lo ha condiviso ha commentato "wo" - "io" in mandarino - in cima al post.

Fig. 11.10 Memi di Kermit postati su Facebook nel sito in Cile: "A volte vorrei andarmene lontano, ma poi mi ricordo che non ho abbastanza nemmeno per coprire il costo di un biglietto e non ci penso più" e "A volte vorrei licenziarmi. Poi mi ricordo che non ho nessuno che mi mantenga e non ci penso più".

Fig. 11.11 Memi di Kermit che circolavano su Facebook a Trinidad.

Fig. 11.12 Immagini che mostrano come l'espressione facciale possa emulare quelle delle emoticon su Snapchat. 


\section{Elenco delle tabelle}

Tabella 4.1 Chi ti ha messo a punto il tuo primo account sui social media? Io stesso - Amici - Genitori - Fratelli - Partner - Figli - Altri parenti - Un addetto di un internet point

Tabella 4.2 Chi posta regolarmente sui tuoi social media? Genitori - Fratelli - Partner - Figli - Amici - Nessuno. Chi tra i familiari e gli amici posta regolarmente sui tuoi social?

Tabella 4.3 Con chi hai discussioni sul modo di usare i social media? Genitori - Fratelli - Partner - Figli - Amici - Altri parenti. Persone con cui si hanno discussioni sul proprio uso dei social media

Tabella 4.4 Con chi condividi la tua password di accesso ai social media? Partner - Genitori - Fratelli - Figli - Amici. Persone con cui gli utenti condividono la loro password di accesso ai media

Tavola 4.5 Distribuzione delle risposte in base all'età. 


\section{Elenco dei collaboratori}

Elisabetta Costa è Postdoctoral Research Fellow presso il British Institute di Ankara (BIAA). Tra i suoi interessi di ricerca ci sono i social media e i media digitali, il giornalismo, la politica e il genere in Turchia e in Medio Oriente.

Nell Haynes è Postdoctoral Fellow presso la Pontificia Universidad Católica de Chile di Santiago. Ha conseguito il dottorato di ricerca in antropologia presso l'American Univerisity. Le sue ricerche toccano temi come la performance, l'autenticità, la globalizzazione, l'identità etnica e di genere in Bolivia e in Cile.

Tom McDonald è Assistant Professor presso il Dipartimento di Sociologica della Hong Kong University. Nel 2013 ha conseguito il dottorato di ricerca presso l'University College of London (UCL). È autore di numerosi articoli scientifici sull'uso di internet e sulle pratiche di consumo in Cina.

Daniel Miller è Professore di Antropologia presso l'University College of London (UCL). È autore e curatore di numerosissime pubblicazioni tra cui si ricordano: Tales from Facebook; Digital Anthropology (curato con $\mathrm{H}$. Horst); The Internet: an Ethnographic Approach (con D. Slater); Webcam (con J. Sinanan); The Comfort of Things; A Theory of Shopping e Stuff.

Razvan Nicolescu è Research Associate presso l'University College of London (UCL), dove ha conseguito il dottorato di ricerca (2013). Formato sia nell'ambito delle telecomunicazioni sia dell'antropologia, ha condotto ricerche etnografiche in Romania e in Italia. Tra i suoi interessi di ricerca rientrano l'antropologia digitale, l'economia politica, la governance, l'informalità, i sentimenti, la soggettività e la normatività.

Jolynna Sinanan è Vice Chancellor's Postdoctoral Research Fellow presso il Royal Melbourne Institute of Technology (RMIT). Tra il 2011 e il 2014 è stata Research Fellow in Anthropologia presso l'University College of London (UCL). Ė autrice, insieme a Daniel Miller, di Webcam. Tra i suoi 
interessi di ricerca: etnografia digitale, nuovi media, migrazioni e genere a Trinidad, in Australia e a Singapore.

Juliano Spyer è dottorando presso il Dipartimento di Antropologia dell'University College of London (UCL). Dopo essersi formato alla ricerca nell'ambito della storia orale, ha conseguito un MSc in Antropologia Digitale presso UCL. È autore del volume Conectado (Zahar, 2007) che indaga gli usi dei social media in Brasile. Nel 2010 è stato consulente digitale per campagna presidenziale di Marina Silva (2010).

Shriram Venkatraman è dottorando presso il Dipartimento di Antropologia dell'University College of London (UCL). Formato nell'ambito delle scienze statistiche, prima di iniziare il dottorato, ha ricoperto posizioni di rilievo presso Walmart negli Stati Uniti. Tra i suoi interessi di ricerca: le tecnologie nei luoghi di lavoro, le culture delle organizzazioni e l'imprenditorialità.

Xinyuan Wang è dottoranda presso il Dipartimento di Antropologia dell'University College of London (UCL). Ha conseguito un MSc in Antropologia Digitale presso lo stesso ateneo. È un'artista di pittura tradizionale cinese e di calligrafia. Ha tradotto in lingua cinese Digital Anthropology (a cura di H. Horst e D. Miller), oltre ad aver contribuito allo stesso volume con un articolo sull'antropologia digitale in Cina. 


\section{Indice degli argomenti}

Abuso, fisico, emotivo, sessuale 172

Accesso ai social media sul posto di lavoro/ norma educativa durante le ore lavorative 58

\section{Pubblicità 53}

da parte di aziende che si rivolgono al pubblico 122

sui social media 122

essere influenzati, online o offline 83

Distribuzione dell'età di rispondenti a sondaggi su siti del settore 94

Tempo speso sui social media/ giochi online 94

L'anonimato dei social media cinesi mancanza di privacy 29 materiale online 61 profili QZone 152

\section{Antropologia 42}

definizione 220

etnografia, 'osservazione partecipativa' 49

siti di settore, politica o migrazione 50

partecipazione, amicizie genuine 49

vivere tra persone diverse 50 fotografia, coinvolgimento di fotografi 197

vantaggi 61

studi di affinità 41

Comportamento appropriato o inappropriato 41

adattarsi alle persone 52

Avatar per diventare amici di sconosciuti, 'maschera' 79

Numero medio di amici sui social media principali

QQ in Cina, Facebook in tutti gli altri siti 64

Generi di comportamento, socializzare sui social media 254

Brasile, espansione delle chiese protestanti

affluenza e potere 226

contributo all'uguaglianza di genere 159

relazioni cordiali offline, relazioni amichevoli sui social media136

distrazione o positività sul posto di lavoro 88

crescita dell'industria turistica159

l'educazione per la mobilità sociale 169

visibilità sui social media per le 
persone LGBT 160

donne, crescita di opportunità lavorative, ancora più visibilità pubblica 159

\section{Siti di settore brasiliani}

attività di adulti sui social media 209

paura delle conseguenze negative in politica 185

calciatori, marchio Nike 85

genitori, i social media come positivi 100

i social media come lo spazio di una persona giovane 138 insegnanti, i social media come 'internet dannoso' 100

matrimonio, mostrarlo sull'account Facebook 172

\section{L'uso dei social media da parte di bambini, età appropriata 86}

\section{Cile}

città di Alto Hospicio, evasione di consumazione 81 ambizione, conforto, supporto per le famiglie 237

le popolazioni di Aymara e Quechua 47

siti di dating online non sicuri 72

famiglia estesa, un solo computer 77

'feria', (mercato) 125

nord, Instagram, Grindr per i gay 161

mancanza di interesse genitoria- le, il coinvolgimento su Facebook 110

modelli di rappresentazione di genere 155

validità di relazioni romantiche 143

l'importanza di Whatsapp per i minatori 139

\section{Mine di bronzo cileno}

comunicazione tramite social media per la famiglia 117

Siti di settore cileni 192

commercio attraverso individui su Facebook 255

ineguaglianza tra le persone 176

meme 211

scuola, foto di eventi 110

crescita della visibilità delle relazioni 224

\section{Siti di settore cinesi}

Storia confuciana, taoista e buddista 238

'classe emergente' 119

dipendenza da social media di lavoratori di fabbriche 91

esaltazione dell'amore e del matrimonio 239

migranti, telefonate giornaliere a lunga distanza 229

divieto di uso di internet cafe 31, 189

\section{Cina industriale}

paragone tra le vite di residenti 
della piccola borghesia 243

spesa digitale 241

relazioni a lunga distanza, partner più interessati online 139 uomini liberi di esprimere il romanticismo 160

uomini della piccola borghesia, prendersi gioco della politica 192-93

un figlio per famiglia, no ai fratelli 67

amicizie online come 'chun' (più pure) 141-42

quello che le persone dovrebbero pensare 236

prevenzione all'accesso a Facebook e Twitter 120

privacy, nessuna aspettativa 226-27

richieste di aiuto finanziario 142 i social media come radice dell'infedeltà 243

i social media per la propaganda 187

donne che condividono selfie 76

\section{Cina, rurale}

l'importanza dell'educazione dei bambini 101

corruzione in politica 138

'interiorità femminile', la sfera domestica 158

post su bambini, amore e matrimonio 198

impatto negativo dei social media sul progresso educativo 101 tempo speso fuori dalla scuola104

Cina, rurale e industriale

discrepanza nel commercio 81 condividere la password, QZone 78

controllo dello stato sull'uso dei social media e di internet 187 88

La paura cinese della disapprovazione degli amici 72

Gli ideali cinesi, antichi e tradizionali 129

Piattaforme cinesi, Renren, simile a Facebook 32

I siti cinesi QQ e QZ, WeChat 30

Accesso commerciale a internet a metà degli anni '90 165

Comunicazione più visuale, Snapchat 215

Spazio conservativo su Facebook 161

Corruzione, regolamentazione locale, inquinamento, stato sociale ineguale 72

Corteggiamento e relazioni romantiche 156

Capitale culturale 171 educazione, beni culturali, qualifiche 166 
Ideali culturali

prospettiva filosofica, livello di educazione 233

\section{Tradizione, antica in Cina}

dare soldi in buste rosse durante i festival, a capodanni e ai matrimoni 128

\section{Il danno dei social media sui giovani} 99

Depressione, insoddisfazione, gelosia, immagine negativa del corpo, solitudine 235

de Tocqueville, A., Democracy in America sull'individualismo 31, 219

Crescita della comunicazione digitale 165

popolazioni sottoprivilegiate socialmente, Cina, India, Brasile 165

\section{Tecnologie digitali}

destabilizzazione delle differenze di genere 150

partecipazione democratica significativa 141

repressione politica 141

riduzione della curva dell'attenzione 135

\section{Distribuzione delle risposte}

percentuale di fotografie postate sui social media 74

domanda su chi gli user consultano quando aggiungono nuovi amici sui social media 67

domande a user che pensavano che avere più amici offline sarebbe risultato in più amici online 65

profili sui social media senza nome reale/foto 79

Crescita del divorzio, siti di settore italiani 243

Mercati domestici in Cina

Taobao sito di shopping online 85

Terremoto in Cile, aprile 2014 l'uso dei social media per le vittime 177

\section{E-commerce}

la preferenza inglese, astratta, impersonale 130 'websites', Flipkart, shopping di magliette, India 127

Disuguaglianza nell'educazione 166

Educazione e possibilità di impiego 119

Educazione e giovani $97-113$

Educazione come argomento sui social media 58

Siti di settore inglese 239

uso di dati commerciali per pubblicità mirata 227 preoccupazione sull'informa- 
zione sui social media nella vita privata 227

Facebook per incrementare vicinanza a lavoro 117

uomini gay, donne lesbiche 162

humour sulla politica 190

tenere le persone alla giusta distanza 229

preoccupazione sulla sorveglianza genitoriale 98

declino nella socialità percepito 139

comunicazione insegnante-scuola tramite social media 109

\section{Facebook 17, 221}

piattaforma pubblicitaria 127

eventi di famiglia 207

buone relazioni 181

fotografie e meme da Trinidad 144

profili, Italia 228

'Amici su Facebook', termine usato in Brasile 135

Lavoratori di fabbrica in Cina, giocare per rilassarsi 75

gruppo svantaggiato 80

nuovi gruppi 221

connessioni sociali online stabili 64

Account falsi, diversi per una sola persona 79
Dominanza femminile, di più siti visuali 37

Ineguaglianze finanziarie in Brasile, Cina, sud dell'India 100

Questioni finanziarie, espressione di intimità in Cina 255

Educazione formale e informale sui social media 179

Forme di comprensione basate sull'empatia 258

'Amico', significato del termine brasiliano colegas 59

Amicizia in America Latina e in Trinidad 136

Amicizie tra uomini online più visibile che tra le donne 154

Gaming consoles

Xbox e Playstation, Farmville 248

Giocare per i giovani in Cina 75

Identità gay 160

Identità geek 67

Genere

differenze tra femminilità e mascolinità 149-163

Differenze di genere nel mondo offline 150 
Segregazione di genere su Facebook, Turchia, sudest 151

Generalizzazione, precarietà, Turchia o Cina 36

Espansione globale dei social media 251

Google, per gli insegnanti, visto come 'internet benefico' 106

Grindr, Tinder, US 33

per maschi omosessuali, piattaforma di dating 72

'guanxi' (relazioni sociali) 137

\section{Felicità}

definizione, motivo, giudizio, emozione 234-35

gratificazione istantanea o grati-

ficazione duratura 233

riconoscimento di 234

emozioni negative o positive, scopo e significato della vita 234

delle relazioni con altre persone 241

dei sociali media, prospettiva antropologica 233

uso dei social media per capire 245

Salute, raggiungimento educativo per la felicità 234

Onore e vergogna nelle società del Mediterraneo 43
Analfabetismo in Brasile 134, 184

Popolazioni illetterate in India, 'conversazioni' visuali 249

Postare immagini di un successo scolastico per i genitori 109-10

Immagini, appropriatezza delle 105

Fotografie di bambini scattate in studi professioni e postate dai genitori su QQ 200

Fotografie di cibo scattate alle riunioni di famiglia nel sud-est della Turchia 207-8

Fotografie che mostrano come le espressioni facciali possono emulare quelle delle emoji in Snapchat 21517

India, sud

presenza su Facebook 201

figli di famiglie povere, comportamenti positivi nei social media 100

comunicazione tra membri della famiglia, WhatsApp 142

scoraggiamento di comunicazioni insegnante-genitore 110 'classe emergente' 119 incoraggiare i figli a usare i social media 101

attività imprenditoriali da casa 127

saluti giornalieri sulle fotografie 204-5

Facebook come piattaforma 
prescelta 127

divieto ufficiale sull'uso dei social media a scuola 107

divieto alle donne non sposate di avere un cellulare 101

restrizioni sul comportamento delle donne 73

social media per gli amici, non per la famiglia 65

marketing sui social media 84 i social media per flirtare 158 uso dei social media dei ricchi 100

famiglie ricche, figli 100

Ineguaglianza di accesso 168

\section{Ineguaglianza}

distribuzione di ricchezza e povertà $165-80$

Instagram e Snapchat, per i giovani 33

Instagram, Facebook, WhatsApp 120

Instagram, sconosciuti benvenuti 21

Internet, comunicazione, informazione, geografie, ineguaglianze 42

Moralità di internet 217

Siti di settore italiani

barriere all'amicizia studente-insegnante 108

alto tasso di disoccupazione 170 limitata comunicazione scuola-genitore tramite social media 111

Socialità italiana offline 65

I social media italiani, una piattaforma pubblica per gli amici 69

Italia

e business 124

frustrazione ed espressione di rabbia sulla politica 193

selfie di giovani donne prima del matrimonio 201

uso del cellulare in classe 103

Imparare attraverso spazi online 97

Popolazioni LGBT 160

Cerimonie del ciclo della vita, fare degli amici 176

Mettere 'mi piace' ai post 229

LinkedIn, assunzioni $\quad 118-19$

Solitudine 219

Mardin, Turchia educazione e possibilità lavorative per le donne 156

le donne negli spazi pubblici 156

Marketing, spazio limitato sui social media 83

Donne sposate in Italia 
i ruoli di mogli e altri, stress 153

\section{Meme}

come 'polizia di internet' 210 circolati su Facebook nel nord del Brasile 209

moralità e umorismo 210-12

rinforzare le norme sociali 210-12

\section{Migranti}

i social media per sfuggire al controllo della famiglia e delle tradizioni 46

Negozi di cellulari, Cina

per avere accesso a internet dagli smartphones 67

Rivoluzione Online 220

Moderno giornalismo popolare, le preoccupazioni dominanti 219

Monetizzazione di WeChat attraverso le 'buste rosse' 129

I soldi come regalo, società occidentali e asiatiche 129

Fare soldi attraverso $i$ social media 82

Moralità in Cina 240

Obbligazioni morali dei genitori nell'educare i figli 106

Società musulmane controllo dei social network delle donne 222

Neo-liberalismo 31

Networks, amici, famiglia, colleghi di lavoro 212

La normativa 41

Nucleo familiare, crescita senza nonni, India 127

Identità offline e online 'reale' e 'virtuale' 146-47

La condizione offline dell'infelicità dei lavoratori di fabbrica cinesi 91

Interazioni offline, rimpiazzare 133

Relazioni offline

dipendenza da relazioni online 143

rimpiazzate 133

Socialità offline, più 'naturale' 135

Persone anziane sui social media, poche 59

Online

appropriatezza delle immagini 245

brutte esperienze, stalking, molestia, compagni infedeli 243

bibliografia 29

bullismo 243

uguaglianza, disuguaglianza offline 178 
giocare sui social media 74

servizi di pagamento ('portafogli digitali') monetizzazione delle piattaforme di social media, Cina 124

corso universitario, The Anthropology of Social Media 259

mondo, separare 133

Open Access, Licenza Creative Commons 60

\section{Codificazione Open Source 257}

Open Source, nuove pratiche digitali 130

Comportamento genitoriale, litigi per supportare i figli $105-6$

Aiuto genitoriale con i compiti, dovere morale italiano 111

Pressioni genitoriali sui giovani 98

Partecipazione 50

Condivisione password tra partners 76-77

Autonomia personale 219

Condivisione di foto 247

Caricamento di foto

sui social media da parte di altre persone 80

Foto di donne $80-1$
Fotografia, tradizionali valori familiari in Cina 216

Piattaforme, sviluppo di nuove 248

Crescita delle piattaforme, Instagram, Snapshot 28

Attivisti politici sui social media 36

Partecipazione politica sui social media influenza da parte di relazioni sociali 184

'partecipazione passiva' 193

Rappresentazione politica, mancanza 166

\section{Politica 181-94}

evasione sui social media con amici 185

intrecciata con i social media 183

cultura comune condivisa 193 sui social media 97

fonte di intrattenimento sui social media 193

I vantaggi della ricerca

educazione sulla conoscenza dell'uso e delle conseguenze sui social media 62

Relazioni prematrimoniali nella Turchia secolare, donne ben educate 151

Presenza sulle piattaforme di social media 
per ragazzi dagli 11 ai 18 anni nelle scuole in Inghilterra 20

Prevenzione dal comunicare online età, rendita familiare, successi educativi, livello di inglese, disabilità, luogo rurale/urbano 167

Privacy e socialità 226

Privacy, impatto dei social media su 76

Importanza della privacy sul commercio inglese 130-1

Privacy in Cina, per le foto 75

La privacy con i social media, WhatsApp 256

Sistemi di messaggeria privata

WhatsApp, Facebook, Viber, Tango, SMS 156

Istruzione privata dei ricchi bambini cinesi 101

Cristianesimo protestante 173

Studi psicologici sui social media 235

Trasmissione pubblica 18

Mezzi di trasmissione pubblica, televisione, radio, giornali 18

Piattaforme rivolte al pubblico, Facebook eQQ 24
Presenza pubblica online, un obbligo 244

QQ 33

nomi anonimi in Cina 78

piattaforme di social media cinesi 78

'gruppi' usati dagli studenti cinesi 103-5

WeChat, no ai post, no ai tag alle foto 108

Disuguaglianza razziale 166

Miglioramento nella lettura e nella scrittura nei social media, India 100

Le ragioni di account multipli su QQ nella Cina industriale 80

Relazioni tra lavoro e non lavoro 115-17

Relazioni tra studenti intimità, dramma, bullismo 104-6

Religione, condividere online 36

Disuguaglianza religiosa 166

'revenge porn'

divulgazione deliberata di materiale intimo 105

I ruoli nei social media, differente 235

Coinvolgimento romantico, nozione di felicità 241 
Soddisfazione data dai social media

studi su Facebook riguardo la positività 241

Consigli self-help per condividere su QQ

per avere successo finanziario, Cina 170

Selfie e narcisismo 203

espressione di sé 198

Selfie

versioni ideali di sé 198

condividere 198

Intimità su Snapchat 138

Social media e nuovi media, cosmopolitismo 99

Ineguaglianze sociali e razziali

problemi dei giovani 170

Capitale sociale, relazioni sociali 166

Fattore sociale di classe 112

Comunicazione sociale e donne anziane 251

Conformità sociale $\quad 230-31$

Frammentazione sociale 219

Gerarchia sociale nelle società asiatiche del sud 46

\section{Social media 17}

accesso, creazione di uguaglianza, Brasile, Cina, India 177 antropologia, cercare e trovare altri aspetti 47

lavori noiosi, Italia 116

peso, risposte positive, Brasile, Turchia 89

obiettivi commerciali, attività morali, Cina rurale 255

aziende, valore delle 120-23

distrazione dall'educazione 112 effetto su popolazioni marginali 253

flirtare per gli uomini, tradire le mogli 156

amicizia con gli insegnanti, appropriata? 107

impatto su educazione, cultura, disuguaglianza 38

incrementare lo stato sociale, popolarità 185

infedeltà 76

isolamento e solitudine139

vita morale, Cina 238

nuovi amici sui social media66

fotografia 200

piattaforma, Nord America, MySpace 32

potenziale per connettersi con estranei 141

stress 242-44

mezzi supplementari per imparare 100

minaccia alla privacy 36

Mobilità sociale 171, 239

migliorare la posizione sociale 166 fattore sociale di classe, cose 'giuste' 166-67 
Norme sociali, nozioni di controllo rispettabile nelle relazioni sociali 150

\section{Relazioni sociali $63-94$}

Separazione sociale 220

Valori sociali, durata $239-40$

India del sud

appropriatezza nel vestire l'abito indiano 53 uso ed effetto 46

Stato della sorveglianza online, Turchia 182

Estranei, contatto con Twitter e Instagram 248

Relazioni superficiali, Tinder o Grindr 242

Taobao, piattaforma commerciale cinese 124

Insultare sui social media $105-6$

Il divieto degli insegnanti sui social media a scuola 107

\section{Tinder in Inghilterra}

social media per il dating 73

\section{Argomenti dei social media}

intimità, appuntamenti, amicizia, identità 36

Trinidad 190-91

coltivazione dell'apparenza esterna 198-99

stato economico

scuole private e social media 102

inglese formale a scuola, dialetto sui social media 109

meno enfasi sui social media 102

farsi degli amici equivale a divertirsi 52

sulla verità di una persona 144 ritorno ai valori tradizionali della comunità 239-40

social media per uso commerciale 127

\section{Turchia, sudest}

grande favore familiare ai social media 102

Facebook conservatore 151

controllo dei mariti sulle mogli 156

giochi come Candy Crush Saga, Ok, Taula 76

donne rinchiuse in casa, l'importanza dei social media 156 importanza della politica 72 essere gay apertamente non è accettabile 160

investimenti di proprietà attraverso Facebook 124-25

incontri omosessuali segreti 160

opportunità per le donne di relazioni sociali 157

chat di giovani uomini e donne 24 
Tweets su Twitter, chiacchiere scolastiche 22, 104-5

Uso di Twitter, per informazione o per chiacchierare 23

University College, Londra, UCL Press 60

Togliere l'amicizia sui social media a causa di post politici 71

Visibilità delle relazioni, incremento di gelosia e sorveglianza 141

Antropologia visuale 197

Associazioni visuali online, auto diffusione 196

Immagini visuali 212

Immagini visuali nella comunicazione 215

Materiale visuale, appropriatezza di 74

Post visuali

$$
\begin{aligned}
& \text { ambiguità } 215 \\
& \text { norme di genere } 154 \\
& \text { foto e meme } 73-4
\end{aligned}
$$

Ricchezza e stato sociale, amici offline in Cina 141

Webcams, 'sempre accese' 228

WeChat, WhatsApp 34

Categorie 'occidentali', razionale contro 'strano' 128

WhatsApp 30,111, 134

la mentalità degli insegnanti indiani 143

nelle classi 21

in piccoli gruppi 248

Why We Post, risultati della ricerca 259

Lavoro e commercio $\quad 115-131$

Scrivere, creare oblio 134

YouTube 27

incremento dell'importanza per l'educazione informale 171 video fatti con i compagni 98

YouTube, troll anonimi linguaggio offensivo contro i politici 190

Video su YouTube, che insegnano qualità lavorative 170 



\section{Antropologia della contemporaneità}

Direzione: Simone Ghezzi, Vincenzo Matera, Luca Rimoldi

Comitato Scientifico: Naor Ben-Yehoyada, Michael Blim, Angela Biscaldi, Elisabetta Costa, Silvia de Zordo, Gabriella D’Agostino, Fulvia D’Aloisio, Ferdinando Fava, Adriano Favole, Paolo Favero, Elizabeth Krause, Alessandro Mancuso, Noelle Molé, Bruno Riccio, Fabio Vicini, Valeria Siniscalchi.

La collana "Antropologia della Contemporaneità" si propone come uno spazio di riflessione, condivisione, dibattito e approfondimento per gli studi antropologici. Interrogarsi sugli assetti sociali e culturali in cui viviamo, a partire dal riconoscimento della simultaneità che avvolge tutti, è il filo conduttore della collana. Ricerche etnografiche contemporanee e classici della disciplina possono contribuire in modi diversi a costruire quella particolare consapevolezza contro-intuitiva che è la forza della disciplina antropologica e che può offrire una prospettiva inattesa da cui leggere situazioni, contesti, conflitti, dinamiche, discorsi, organizzazioni, pratiche che richiedono di essere rese meno opache.

La collana adotta un sistema di double-blind peer review. 

Come il mondo ha cambiato i social media è il volume complessivo di comparazione dei risultati di un'ampia indagine etnografica, coordinata da Daniel Miller, dall'eloquente titolo 'Why We Post'. Nove ricercatori, incluso Miller, hanno trascorso 15 mesi sul campo, in diversi paesi del mondo (Italia del sud, Turchia sudorientale, due siti in Cina, area rurale e area industriale, Trinidad, Inghilterra, India del sud, Cile settentrionale e Brasile) a osservare e studiare, con un approccio etnografico, i modi in cui le persone usano i social media. È un fatto indiscutibile che i social sono entrati nella nostra vita con prepotenza, in modo capillare, per certi aspetti invasivo. Con un linguaggio fluido, talvolta anche colloquiale, il lettore è condotto all'interno di un ambito che gli sembra di conoscere, se non altro perché ne siamo tutti, più o meno, utenti, scoprendo però quanto di valori, di comportamenti culturalmente codificati, di 'polizia morale' ci sia dentro i social media. L'approccio qui presentato parte infatti da un'idea un po' diversa rispetto a quelle più diffuse, e avvalorata nel corso della ricerca: se è indubbio che i social media hanno cambiato il mondo, la questione più interessante riguarda però il modo in cui il mondo li ha cambiati.

Daniel Miller è professore di Antropologia all'UCL (University College Londra). Elisabetta Costa è Postdoctoral Research Fellow presso il British Institute di Ankara (BIAA). Nell Haynes è Postdoctoral Fellow alla Pontificia Universidad Católica di Santiago del Cile. Tom McDonald è Assistant Professor nel Dipartimento di Sociologia della University of Hong Kong. Razvan Nicolescu è Research Associate nell'UCL, dove ha conseguito il dottorato di ricerca nel 2013. Jolynna Sinananis è Vice Chancellor's Postdoctoral Research Fellow presso il Royal Melbourne Institute of Technology (RMIT). Juliano Spyer, Shriram Venkatraman e Xinyuan Wang sono dottorandi in Antropologia presso I'UCL di Londra. 